FINAL REPORT

(Volume I - Study Results)

\title{
LASER FUSION STUDY
}

June 1975

Prepared for:

Univer sity of California Lawrence Livermore Laboratory Livermore, California 94550

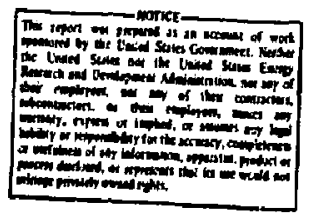

Electro-Optical and Data Systeme Group

AEROSPACE GROUPS

Hughes Aircraft Company - Culver City, California 
1. INTRODUCTION AND SUMMARY . . . . . . . . . 1-1

Study Objectives and Problems Addressed by

Hughes $\ldots \ldots \ldots \ldots \ldots \ldots \ldots \ldots$ 1-1

Overview Description of Alignment System ....... $1-2$

Alignment System Components . . . . . . . . . 1-6

Alignment System Features ... . . . . . . . . . 1-9

2. SYSTEM DESGRIPTION $\ldots \ldots \ldots \ldots \ldots \ldots \ldots \ldots \ldots \ldots$ 2-1

System Performance Requirements .......... 2-1

Syrstem Phy gical/Functional Description ........ 2-13

Operating Procedure . . . . . . . . . . . . 2-29

Interconnection and Interfacing . . . . . . . . . 2-49

3. POINTING AND FOCUS SENSING $\ldots \ldots \ldots \ldots \ldots \ldots \ldots$. . . . . .

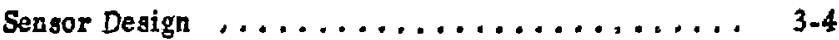

4. THE FOCUSING IENS CENTERING SENSOR $\ldots \ldots \ldots \ldots$. . . 4-1

Requirements $\ldots \ldots \ldots \ldots \ldots \ldots \ldots \ldots \ldots \ldots \ldots$ 4-1

Sensing Technique and Description ........... 4-1

Predicted Performance .............. 4-1

5. PINHOLE SENSOR .................... 5-1

Eequirements ................ 5-1

Sensing Technique and Phy sical Description ...... 5-1

Performance Predictions ................ 5-4

6. Laser chain CENTERING SENSORS ............. 6-1

Requirements ................. 6-1

Apodize Aperture Sensor Description .......... 6-1

Last Spatial Filter Sensor Description ... . . . . . . 6 6-4

Predicted Performance ............... 6-5

7. PATHLENGTH SENSOR ................. 7-1

Pathlength Equalization . . . . . . . . . . 7-1

Proposed Instrumentation . . . . . . . . . . . . . . 7-1

Alignment ................... $7-2$

Mathematical Relationships ............. $7-4$

Estimated Accuracy ................. 7-6

Alignment Lamer Requirements . . . . . . . . . 7 7

Potential Problems ................. 7-8

8. TWO AXIS MIRROR GIMBAL ............... 8-1

Functional Definition . . . . . . . . . . . . $8-1$

Performance Requirements ............. 8-1 
CONTENTS (Continued)

Environmental Conditions $\ldots \ldots \ldots \ldots \ldots \ldots \ldots$ 8-2

Gimbal Techniques ................ 8.3

Actuating Techniques $\ldots \ldots \ldots \ldots \ldots \ldots \ldots \ldots$. $8-10$

Bascline Design ................ 8-15

Test Model ................. 8-21

9. FRONT-END POSITIONING $\ldots \ldots \ldots \ldots \ldots \ldots \ldots \ldots$ 9-i

Futctional Description ............... 9-1

Pet formance Requirements ............ 9. 9-2

Adjustment Techniques .............. 9. 9.2

Baseline Design ................ 9-5

10. APERTURE INDEXING DEVICE ............. 10-1

Function and Requirements ........... 10-1

Design .................... 10-1

11. OPTICAL DESIGN AND ANALYSIS ........... 11-1

System Synthe sis and Conitiguration Evaluation

Overview ...................... 11-2

Sensor Design Study ................. 11-9

Configuration Studies and Tradeoffs $\ldots \ldots \ldots \ldots$ 11-12

Sensor Design Study . . . . . . . . . . . . . . 11-31

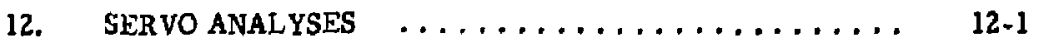

Servo Design Requirements . . . . . . . . . . . 12-1

System Synthesis . . . . . . . . . . . . . 12-1

Spring Ratio Servo Analysis ... . . . . . . . 12-40

Servo Hardware Design .............. 12-56

Summary of Conclusions ........... 12-63

13. BIREFRINGENT SENSOR ................. 13-1

Measuring Angle with Bircfri.jgent Crystals . . . . 13-4

Savart Plate................... 13-6

Principle of Operation ............... 13.9

Error Detection and Analysis of Polarization

State $\ldots \ldots \ldots \ldots \ldots \ldots \ldots \ldots \ldots \ldots \ldots \ldots . . .13 .11$

Optical Analysis for Savart Plate Sensor ...... 13-16

Birefringent Sensor Design ........... 13-19 


\section{LIST OF ILLUS'SRATIONS}

Figure

Page

1-1 Simplified alignment system schematic $\ldots \ldots \ldots \ldots \ldots \ldots$ 1-4

$1-2$ Alignment sequence $\ldots \ldots \ldots \ldots \ldots \ldots \ldots \ldots \ldots \ldots \ldots$

2-1 Pointing error caused by centering error and offset focusing ..................... 2-5

2-2 Sinplified functional schematic of loser alignment control system ................... 2-15

2-3 Master oscillator and aligament laser to pinhole alignment .................... 2-16

2-4 Apodized aperture centering implementation ........ 2-17

2-5 Laser chain centering implemeniation .......... 2-18

2-6 Manual control block diagram for apodized aperture and spatial filter centering $\ldots \ldots \ldots \ldots \ldots \ldots \ldots . \ldots . \ldots .2-20$

2-7 Remote control of apodizes aperture and last spatial filter centering with crosscoupling compensation ..... 2-21

2-8 Location of centering sensors . . . . . . . . . . . 2-22

2-9 Centering sensor location in truncated beam profile .... 2-22

2-10 Control function for pure transverse control of beam ... 2-23

2-11 Location of pointing and focus sensor in optical train ... 2-24

2-12 Control function for pure angular control of team ...... 2-24

2-13 Pointing and centering control impiementation ....... 2-26

2-14 Master console control panel ............... 2-31

2-15 Portable test box ................... 2-33

2-16 Alignment laser to pin hold alignment ......... 2-34

2-17 Apodized aperture and laser chain alignment ....... 2-36

2-18 Alignment for sensor and system ...........

$2-19$ Final pointing/centering/focusing $\ldots \ldots \ldots \ldots . \ldots .40$

2-20 Path length measurernent $\ldots \ldots \ldots \ldots \ldots \ldots \ldots \ldots \ldots$ 2-46

2-2! Master oscillator to pinhole alignment .......... 2-48

2-22 System functional diagram ............... 2-51

3-1 Baseline optical configuration for Location of angle and focus senvor ...................... 3-2

3-2 Relation of pointing and focus sensor beameplitter and setro $\ldots \ldots \ldots \ldots \ldots \ldots \ldots \ldots \ldots \ldots \ldots \ldots$ 


\section{LIST OF ILLUSTRATIONS (Continued)}

Figure

Page

3-3 Concept for sensing focus . . . . . . . . . . . . 3-4

3.4 Generation of focus error gignal $\ldots \ldots \ldots \ldots \ldots \ldots . . .5$

3-5 Parametric variation of modulation index ........ 3-6

3-6 Through-focus evaluation of energy passing through circular aperture centered at nuli balance of quadrant detector for 1 coma input error . . . . . . . . . . 3-7

3-7 Selected focus detection scheme utilizing single detector and chopper ................. 3-10

3-8 Centroid shift as a function of focus with one wave of come ....................... 3-12

3-9 Pointing and focus sensor layout $\ldots \ldots \ldots \ldots \ldots \ldots \ldots . . \ldots$ 3-17

3.10 Pointing and focus sensor $\ldots \ldots \ldots \ldots \ldots \ldots \ldots \ldots$ 3-18

4-1 Centering sensor configuration .............. 4-2

4-2 Final lens centering sensor, four/cbanuelo ......... 4-2

5.1 Pintole sensor configuration .............. 5-1

$5-2$ Pirbole sensor ..................... $5-2$

5-3 Alternate method of combining of master oncillator and alignment laser beams ................ 5-3

6.1 Apodized aperture sensor optical layout ........ 6-2

6-2 Apodized aperture centering sensor location ....... 6-3

$6-3$ Last spatial filter centering sensor .......... 6.5

$7-1$ Froposad Configuration .............. $7-2$

$7-2$ Principle of operation. .............. $7-3$

8-1 Quzd pod gimbal technigue................ 8.3

8.2 Center of rotation for quad pod gimbal. . . . . . . . 8-4

8-3 Double pirot gimbal ................ 8-5

8-4 Cross-ntrap flexure pivot gimbal $\ldots \ldots \ldots \ldots \ldots \ldots$ 8-6

8-5 Two axis gimbal using gimbal ring .......... 8-6

8-6 Two axis flexure column ............... $8-7$

8-7 Bendix data sheet. . . . . . . . . . . . . $8-8$

8-8 Linkage reduction device (stecring minror layout) . . . . 8. 8-11

8-9 Linkage reduction device schematic ciagram ... . . . 8-16 
LIST OF ILLUSTRATIONS (Continued)

Figure

Page

8-10 Linkage reduction device. . . . . . . . . . . . . 8-12

$8-11$ Inch-worm derice $\ldots \ldots \ldots \ldots \ldots \ldots \ldots \ldots . \ldots .13$

8-12 Spring ratio reducticn technique . . . . . . . . 8 8-14

8-13 Spring ratio actuator device............. 8-17

8-14 Mechanical design - sp:ing ratio actuator device..... 8-18

8-15 Two-Axis mirror actuating assembly .......... 8-19

8-16 Mechanical design - test model device......... 8-22

8-17 Test Configuration . . . . . . . . . . . . . . 8-22

9-1 Path length adjustment concept.............. 9-3

9-2 Mechanical design - path length adjustment device..... 9-4

9-3 Front end positioner (FEP) device .......... 9-6

10-1 Aperture indexing device............... 10-2

11-1 Five ways of reflecting the alignment beam ....... 11-3

11-2 Alignment configuration with sensor at input $\ldots \ldots \ldots \ldots \ldots 11 . \ldots \ldots$

11-3 Alignment configuration with sensor near focusing

11-4 Alignment configuration with image on mask at edge of focus less $\ldots \ldots \ldots \ldots \ldots \ldots \ldots \ldots \ldots \ldots \ldots \ldots$

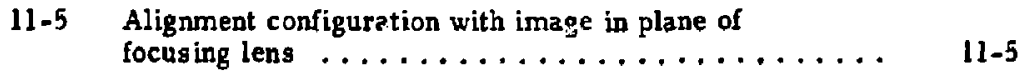

11-6 Aignment configuration with focus at surface of

11-7 Baseline alijnment configuration ........... 11-5

Baseline coufiguration of sensor package and
tradeoff study parameters $\ldots \ldots \ldots \ldots \ldots \ldots \ldots$

11-9 Three variations of baseline configuration ......... 11-10

11-10 Relation nip of focused spot locus with respect to the annulus mask as seen from detector ........... 11-15

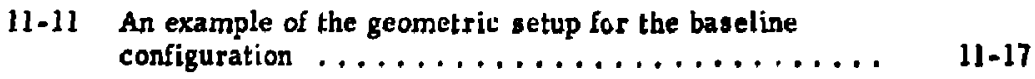

11-12 A simplified version of the example shown in
Figure $11-1] \ldots \ldots \ldots \ldots \ldots \ldots \ldots \ldots \ldots \ldots$ 
LIST' OF ILLUSTRATIONS (Continued)

Figure

Page

11-13 Beam diameter at focusing lens as a function of image dist:nce for various ball diameters .......... 11-23

11-14 F/ro. as a function of image distance for various ball diameters $\ldots \ldots \ldots \ldots \ldots \ldots \ldots \ldots \ldots$

11-15 Diffration spot diamerer as a function of image distance for various ball diameters ................. 11-25

11-16 Relative peak irradiance as a function of image distance for various ball diameters

11-17 Beam diameter at 26 meters from focusing lens as a function of image distance for various ball diameters $\ldots \ldots \ldots \ldots \ldots \ldots \ldots \ldots \ldots$

11-18 F-number as a function of image distance for various ball diameters $\ldots \ldots \ldots \ldots \ldots \ldots \ldots \ldots \ldots$

11-19 Diffraction spot diameter as a function of image distance for various ball diameters ......... 11-29

11-20 1sometric view of the aperture irradiance function .... 11-33

11-21 Flow chart for diffraction evaluation of system (b) ... 11-36

11-22 Isometric view of point spaced function with id coma input wave aberration ................. 11-40

11-23 Sectional views of point spread function with $1 \lambda$ coma irput wave aberration ................ $11-41$

11-24 Isometric view of wavefront deformation with is coma.. 11.41

11-25 Sectional views of point spread functions with and without $\lambda / 2$ coma input wave aberration ........ 11-42

11-26 Graphs of peak irradiance on axis versus defocus for different input aberrations .............. 11-42

11-27 A comparison of peak irradiance versus defocus for cases with and without transverse focal shifts . . . . . . 11-43

11-28 A comparison of wave aberration of system (b) and system (c) . . . . . . . . . . . . . . . . . . . . 11-44

11-29 A comparison of wave aberration maps of system (b) with $2 \mathrm{~mm}$ ball and system (c) with $1 \mathrm{~mm}$ ball ...... 1l-45

11-30 Plots of Y-alice through PSF at different defocused image planes $1,2,3,4$ and 5

11-31 Plots of KET in $\mathrm{Y}$-direction at different defocused image planes $1,2,3,4$ and $5 \ldots \ldots \ldots \ldots \ldots \ldots$ 


\section{LIST OF ILLUSTRATIONS (Continued)}

Figure

Page

11-32 Graph of peak irradiance verslis defocus for $1 \lambda$

11-33 Plots of $Y$-slice through PSF at different defocused image planes $1,2,3,4$ and $5 \ldots \ldots \ldots \ldots \ldots$

11-34 Plots of LSF in $Y$-direction at different defocused image planes $1,2,3,4$ and $5 \ldots \ldots \ldots \ldots \ldots \ldots$

11-35 Plots of KET in X-direction at different defocused image planes $1,23,4$ and $5 \ldots \ldots \ldots \ldots \ldots \ldots$

11-36 Plats of $\mathrm{KI} T$ in $\mathrm{Y}$-direction at different defocused image planes $1,2,3,4$ and $5 \ldots \ldots \ldots \ldots \ldots \ldots$

$11-52$

11-37 Plots of $X$-slice through PSF at different defocused image planes $1,2,3,4$ and $5 \ldots \ldots \ldots \ldots \ldots \ldots$

11-38 Plats of $Y$-slice through PSF at differ ent defoclised image planes $1,2,3,4$ and $5 \ldots \ldots \ldots \ldots \ldots$

11-39 Plots of LSF in X-direct on at different defoensed image planes $1,2,3,4$ and $5 \ldots \ldots \ldots \ldots \ldots$

11-40 Plots of LSF in Y-direction at different defocused image planes $1,2,3,4$ and $5 \ldots \ldots \ldots \ldots \ldots \ldots$

11-41 KET plots in $\mathrm{Y}$-direction at tifferent defocused image planes 1, 2, 3, 4 and $5 \ldots \ldots \ldots \ldots \ldots \ldots \ldots$

11-42 Graph of equivalent energy centroid shift at target versus defocus at target $\ldots \ldots \ldots \ldots \ldots \ldots \ldots \ldots$

li-43 Graphs of normalized energy incident on detector vergus defocus for the perfect input case and the $1 \lambda$ coma ( $p-p)$ case vith detector located (1) at position with null shift corrected, and (2) on axis $\ldots \ldots \ldots \ldots \ldots \ldots \ldots$

11-44 Graphs of normalized energy incident on detecior versus defocus for the perfect input case and the i $\lambda$ coma (p-p) case with detector located (1) at position with null shift correctea, and $(2)$ on axis $\ldots \ldots \ldots \ldots \ldots \ldots$

11-45 Graphs of normalized energy incident on detector versus defocus for the perfect input case and the $1 \lambda$ coma (p-p) case with detector located (1) at position with null shift corrected, and (\&) on axis $\ldots \ldots \ldots \ldots \ldots \ldots \ldots$

1i-46 Graphs of normalized energy incident on detector versus defocus for the perfect input case and the $I \lambda$ coma (p-p) case with detectur located (1) at position with nul! shift corrected, and $(2)$ on axis $\ldots \ldots \ldots \ldots \ldots \ldots \ldots$ 


\section{LIST OF ILLUSTRATIONS (Continued)}

Figure

Page

11-47 Graphs of n-malized energies contained within four focus detector radi as a function of defocus ....... 11-64

11-48 Fiots of LSF in $\mathrm{Y}$-direction at different defocused image planes $1,2,3,4$ and $5 \ldots \ldots \ldots \ldots \ldots \ldots \ldots \ldots \ldots$

11-49 Plots of KET in X-direction at different defocused image planes $1,2,3,4$ and $5 \ldots \ldots \ldots \ldots \ldots \ldots \ldots$

11-50 Plots of KET in Y-direction at different defocused image planes $1,2,3,4$ and $5 \ldots \ldots \ldots \ldots \ldots \ldots \ldots \ldots \ldots$

11-51 Through focus iso-irradiance contour plots of PSE ... 11-68

11-52 Graphs of transverse null shift versus defocus for two nutating input beams ............... 11-69

12-1 Basic position servo loop $\ldots \ldots \ldots \ldots \ldots \ldots \ldots \ldots . \ldots \ldots$

$12-2$ Frequence response for equation $(12-7) \ldots \ldots \ldots \ldots .12-4$

12-3 Bandwidth as a function of friction torque at load for linear servo with rigid drive $\ldots \ldots \ldots \ldots \ldots \ldots \ldots . \ldots . \ldots$

12-4 Bang-bang controller with rigid load ........ 12-6

12-5 Miechanical diagram of flexible drive with friction ... $12-7$

12-6 Block diagram of spring mass model of Figure 12-5 .. 12-8

12-7 Plot of bandwidth versus motion reduction ratio of linear rigid body servo with a!l friction located

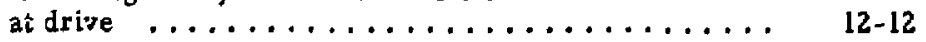

12-8 Mechanical lumped spring mass diagram ........ 12-13

12-9 Block diagram of Figure $12-8 \ldots \ldots \ldots \ldots \ldots \ldots \ldots$ 12-16

$12-10$ Mechanical diagram of spring ratio system ....... 12-20

12-11 Spring servo load block diagram ............ 12-22

$12-12$ Block diagram of simulation ............. 12-27

12-13 Motor position and load position as a function of time $\ldots \ldots \ldots \ldots \ldots \ldots \ldots \ldots \ldots \ldots \ldots \ldots \ldots . \ldots \ldots$ 12-28

12-14 Position as a function of time for load with lamping ratio of 0.01 and $1 \mu$ rad command ........... 12-29

12-15 Position as a funct: $n$ of lime for load with damping of 0,1 and $l \mu$ rad command $\ldots \ldots \ldots \ldots \ldots \ldots \ldots, 12-30$

12-16 Position as a function of time for load with damping ratio of 0.5 and $1 \mu$ rad command 
Figure $\quad$ Fage

12-17 Block diagram of servo used to deterrime pulse

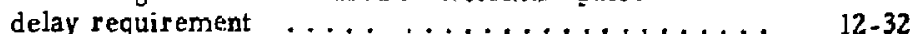

12-18 Motor and load position as a fuctetion of $t$ ime for controlled pulse rate . . . . . . . . . . . 12.34

12-19 Motor and load position as a function of time for controlled pulse rate ............... 12-35

12-20 Motor and load position as a function of time for controlled pulse rate ................ \$2-36

12-21 Motor position and load position as a function of time for fixed pulse rate of $\delta \omega \ldots \ldots \ldots \ldots \ldots \ldots$ 12-37

12-22 Motor position and load position as a function of time for controlled pulse rate ............. 12-38

12-23 Motor and load position as a function of time for $\omega_{n}=$ $10 \mathrm{~Hz}, \delta=0.05 \ldots \ldots \ldots \ldots \ldots \ldots \ldots \ldots$

12-24 Mechanical diagram illustrating control parameters .................... 12-41

12-25 Linearized magnetic and electromagnetic spring rate . . 12-43

12-26 Speed torque curve $\ldots \ldots \ldots \ldots \ldots \ldots \ldots \ldots \ldots \ldots$ 12-44

12-27 Position servo block diagram ............. 12-49

12-28 Simplified position servo block diagram ........ $12-50$

12-29 Motor position, load position, and estimated load rate for $3 \mu \mathrm{rad}$ command $\pm 0.25 \lambda$ rad deadspace, 0.1 load damping, and a maximum pulse rate of 125 pulses per second .................... $12-52$

12-30 Automatic control of centering and coarse pointing .... 12-53

12-31 Feed forward of error signal ........... 12-53

12-32 Simplified diagram of positive feedback path of pointing servo ................... 12.54

12-33 Root locus of parallel paths of pointing servo and centering bearn .................. 12.55

12-34 Response to a 1 mrad disiurbance for 0.01 second into the centering servo and $-1 \mathrm{mrad}$ into the pointing serve . $12-35$

12-\$5 Servo hardware block diagram ............ 12-51 


\section{LIST OF ILLUSTRATIONS (Continued)}

Figure

13-1 Parallel input-output rays focused at center of

13-2 Sensor functional schematic . . . . . . . . . . 13-2

13-3 Fointing and focus sensitivity as a function of sensor position ................... 13-2

13-4 Sma!l sensor positioned in front of final aperture .... 13-3

13-5 Return angle errors for surrogate target displacements . 13-3

13-6 Angle measurement by polarization change ........ 13-4

13-7 The ray-velocity surface $\ldots \ldots \ldots \ldots \ldots \ldots \ldots \ldots$ 13-5

13-8 Isochrornatic pattern seen through crossed polarizers ...................... 13-6

13-9 Savart plate construction and characteristics ...... 13-7

13-10 Relativi retardation and signal strength as a function of angle oi iricidence $\ldots \ldots \ldots \ldots \ldots \ldots \ldots \ldots \ldots$. 13.8

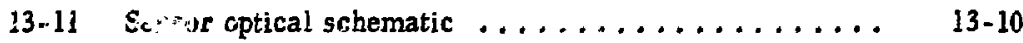

13-12 The Poincare sphere, showing the general significance of the different areas and the specification of the general point $P$ in teims of the angle $2 \lambda$, measured clockwise from $H$, and $2 \omega$, measured downward from the equator $\ldots \ldots \ldots \ldots \ldots \ldots \ldots \ldots \ldots \ldots \ldots \ldots \ldots \ldots \ldots .12$

13-13 Use of the Poincare sphere in predicting the effect of a $90^{\circ}$ linear retarder $R$ on a linearly polarized beam $P$.. $13-14$

13-14 Poincare sphere analysis of birefringent sensor ..... 13-15

13-15 Savart plate sensor unit for detecting misalignment and fozusit: errors .................. 13-16

13-16 Definitions of $\Delta_{E L}$ and $\Delta_{A Z} \ldots \ldots \ldots \ldots \ldots \ldots \ldots, 13-17$

13-17 input wavefronts for Savart plate study ......... 13-18

13-18 $\Delta a$ as a function of relative radial position at entrance pupil perfect input wavefront ............. 13-18

13-19 $\Delta \alpha$ as a function of relative radial position at entrance pupil ........................

13-20 Preliminary Mechanical layout of the birefrigigent serisor 


\section{LIST OF TABLES}

Table

Page

1-1 Primary specifications for alignment system ... . . . . 1-2

1-2 Components required for a 20 chanrel alignment system ....................... $1-3$

2-1 Alignment accuracy specifications.......... 2-1

2.2 Functions required of alignment system......... 2-2

2,3 Pinhole lens centering error allocation ......... 2-2

2-4 Focal point to pinhole erzor allocation......... $2-2$

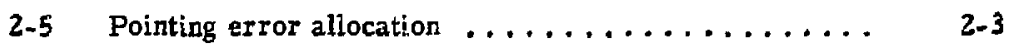

$2=6$ Centering error allocation $\ldots \ldots \ldots \ldots \ldots \ldots \ldots \ldots$

2-7 Last spatial filter centering error allocation ....... $2-3$

2.8 Apodizen aperture centering error allocation ....... $2-4$

2.9 Path length error allocation. . . . . . . . . . . . 2-4

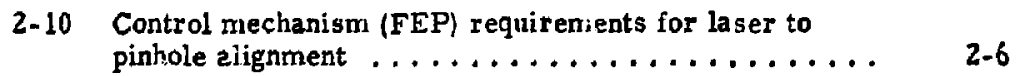

2-11 Control mechanism and (FEP) requirements for laser

2-12 Path length alignment............... $2-8$

2.13 Front end alignment (FEP) speciŕication. . . . . . . . 2-8

2-14 Control mechanism requirements $f o r$ the centering and pointing mirrors $(0.395 \mathrm{~m}$ focal distance) $\ldots \ldots \ldots . . .2-9$

2-15 Mirror gimbal requiraments .............. 2-10

2-16 Laboratory environmental conditions ......... 2-10

2-17 Functions required of alignment system ........ $2-14$

$2-18$ System interconnection summary ........... $2-50$

3-1 Pointing and focus zensing goals ............ 3-1

3.3 Focus parameter summary ............. 3.9

3-3 Pointing and focusing design parameters ........ 3-14

3.4 Point and focus sensor performance .......... 3-15

3.5 Alignment sensitivity for pointing and focus sensor . . . 3. 36

$4-1$ Centering sensors .................. 4-3 


\section{LIST OF TABLES (Continued)}

Tatle

Page

5-1 Design parameters and performance summary ...... 5.4

6-1 Error allocation requirements............ 6-1

6-2 Design parameters and performance summary. . . . . 6-6

7-1 Alignment laser parameters . . . . . . . . .

11-1 Trangmitted energy at four locations on the mask as a furction of ball decentering .............. $11-16$

11-2 Characteristics of focusing lenses used in baseline configuration. ....................

11-3 Results of parametric tradeoffs with $f / 640$ input beam civergence, $1.0 \mathrm{~nm}$ diameter ball (For cases (A) through (D), the focusing lens used is $\mathrm{f} / 2$; for cases $(\mathrm{C})$ and $(\mathrm{H})$, it is $\mathrm{f} / 1.586 .1 \ldots \ldots \ldots \ldots \ldots \ldots \ldots \ldots$

$11-4$ Aberration values for simulating the input wavefront ...

11-5 Sensitivity of sensor package to collimated input beam steering; with $f / 2$ focusing lens and $I$ min diameter ball

11-6 Sensitivity of sensor package to lateral displacements of the $f / 2$ focusing lens; with collimated input beam and

11.7 Sensitivity of seneor package to its own lateral displacements; with collimated input beam, $\mathrm{f} / 2$ focusing lens and $1 \mathrm{~mm}$ diameter ball ................

11-8 Sensitivity of sensor package to axial movements of the $f / 2$ focusing lens; with collimated input beam and $1 \mathrm{~mm}$ diameter ball . . . . . . . . . . . . . .

11-9 S ngitivity of sensor package to its own axial movements; w. th collimated input beam, $1 / 2$ focusing leng and $1 \mathrm{~mm}$ di.meter bail

11-10 Sensitivity of beam centering detection to senser package positioning; with $f / 640$ divergent input beam, $f / 2$

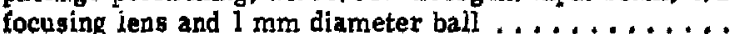

11-11 Sensitivity of sensor package to movements of the surrogate target; with $\$ / 640$ divergent input beam and f/l.586 focusing lens

12-1 Summary of electro-mechanical garameters. . . . . . 12.46

12-2 Relative advantages of SCDT . . . . . . . . . . . 12-57

13.1 Potential Savart plate material summary . . . . . . . . . 13-9 


\section{INTRODUCTION AND SUMMARY}

Hughes Aircraft Company is pleased to submit this final report of the Laser Fusion System Analysis Study (Subcontract 5379325) reflecting activities and accomplishments performed in concert: with the Lawrence Livermore Laboratory. Primary study activities began on December 3, 1975 with a preliminary technical interface meeting at Livermore and concluded with a final technical review at Livermore on May 8, 1975.

\section{STUDY OBJECTIVES AND SROBLEMS ADDRESSED BY HCGHES}

The primasy goal of this study was to devise, evaluate, and conceptually design a complete, end-to-end, alignment system capabie of handling 30 to 32 Shiva amplifier chains to specified accuracies in space and time. A secondary goal was to accomplish tha primary goal with an acceptably low development and procurement cost and with an acceptably high day-after-day performance reliability. This report presents such a system: it is comprised of sensors, actuating mecharisms, controls, and displays that perform well within the current art-state. The components of which they are constructed are readily procurable- often as catalog items.

Early in the study, it was decided that implementation of the final lens focus motion would remain within the province of the Lawrence Livermore Laboratory because the lens is an integral part of the combustion chamber. The sensing of focus error, however, remained a subject of the Hughes investigation.

The conceptual design and analysis of the alignment system and its components were to proceed to a level of detail that:

1. Demonstrate, without equivocation, that the proposed system is capable of performing the alignment tasks, and

2. Allow cost estimates to be made for,

- design and development

- prototype fabrication and teat

- procurement of 20 to 32 chansels

- integration and teat at the Shiva facility. 
The primary performance specifications for the alignment system are shown in Table 1-1.

Table 1-1. Primary specifications for alignment system

Align 20 to $320.5 \mathrm{~K}$-joules laser pilses on target

- Accuracy of $\pm 2 \mu \mathrm{m}$ radially (point:ing)

- Accuracy of $\pm 7 \mu \mathrm{m}$ arially (focus)

- Accuracy of \pm 10 psec ( $\pm 3 \mathrm{~mm}$ path length)

Beyond these primary performance specifications, there are many subsidiary requirements and design constraints which are related to the performance of the high power laser itself. These requirements are dis. cussed in Section 2 and will only be touched upon here to the extent needed to understand the overall function of the alignment system.

\section{OVERVIEW DESCRIPTION OF ALIGNMENT SYSTEM}

The proposed alignment system is assembled from six different sensor types, four different mecharism types, an alignment laser, a master control and display panel assembly, and a portable display/control box. Table 1-2 lists these items and also indicates the number required of each item for a 20 arm Shiva.

In the following subsection, a brief physical description and principle of operation description of each system component is presented along with the specific role it plays in performing an alignment. In this section, however, an overview description of the total alignment system operating as a system is presented.

The alignment system that has evolved as a result of this study begins with the master oscillator and ends with a surrogate target. The groper alignment of all Shiva elements, with the exception of those high power laser elementa that lie between the apodized aperture and the last spatial filter, is accomplished with this system. The alignment of those inner elements is within the purview of Lawrence Laboratory personnel. 
Table 1-2. Components required for a 20 channel alignment system

\begin{tabular}{|l|c|}
\cline { 2 - 2 } \multicolumn{1}{l|}{} & $\begin{array}{c}\text { No. Required Per } \\
20 \text { Charnel System }\end{array}$ \\
\hline Sensors & 20 \\
Pointing and focusing & 80 \\
Final lens centering & 20 \\
Apodized aperture centering & 20 \\
Laser chain centering & 1 \\
Pinhole (master oscillator to alignment laser) & 1 \\
Path length & 40 \\
\hline Mechanisms & 22 \\
Two axis mirror gimbal & 1 \\
Front end positioner (FEP) & 1 \\
Aperture indexing device & 1 \\
Shutters & \\
\hline Miscellaneous & \\
Alignment laser & \\
Master control/display panel assembly & \\
Portablo control/display box & \\
\hline
\end{tabular}

For an overview of the alignment approach, the Shiva system is presented as four separate assemblies whose positional interrelationships must be established and maintained. These assemblies are illustrated schematically in Figure $1-1$ and tabulated below.

1. The front end structure consists of the master oscillator, the alignment laser, the first Shiva pinhole and other aligament system components. This assembly establishes parallelism and translational coincidence between the master oscillator beam and the alignment laser beam. As illustrated in the figure, it also has the capability of moving these beams in angle and transiation. 


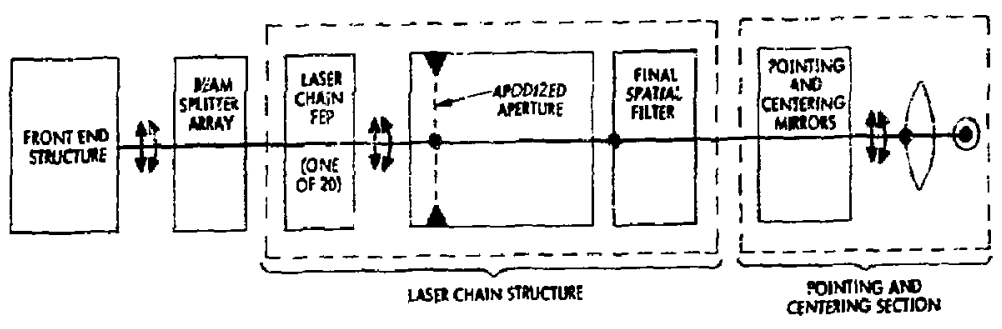

Figure 1-1. Simplified alignment system schematic

2. The beam splitting and folding mirror array is passive as far as the alignment system is concerned. It is, towever, considered to be a potential source of misalignment of any one or all of the laser chains.

3. Any one of the laser chain assemblies begins with its particular front end positioner (FEP) which provides beam translation and beam pointing capability. This FEP is followed by the apodized aperture, the active laser elements and finally the last spatial filter.

4. The pointing and centering section consists of the gimbaled centering mirror and the gimbaled pointing mirror which in combination, provide beam pointing and translation capability. The final focusing lens and the surrogate target are also part of this section.

In Figure 1-1, the large black dots represent the centers of key elements through which the laser beam must pas to achieve alignment. The arrows indicate beam translation and pointing capability.

Figures $1-2 a, b, c, d$ and $e$, illustrate an alignment sequence which begits with the alignment laser beam exiting the front end structure and striking the apodized aperture off center (Figure 1-2a).

1. The laser chain FEP translates the beam as shown in Figure $1-2 b$, so that it passes through the center of the apodized aperture. 
a)

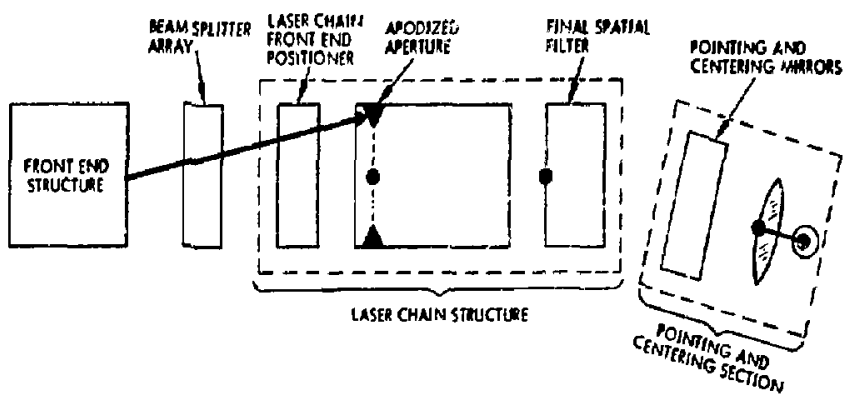

b)
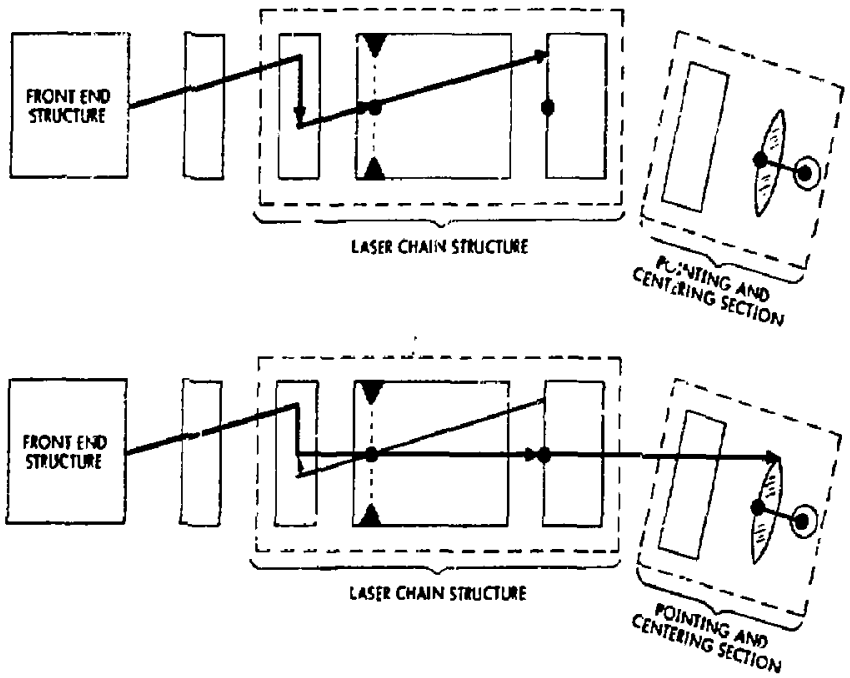

4

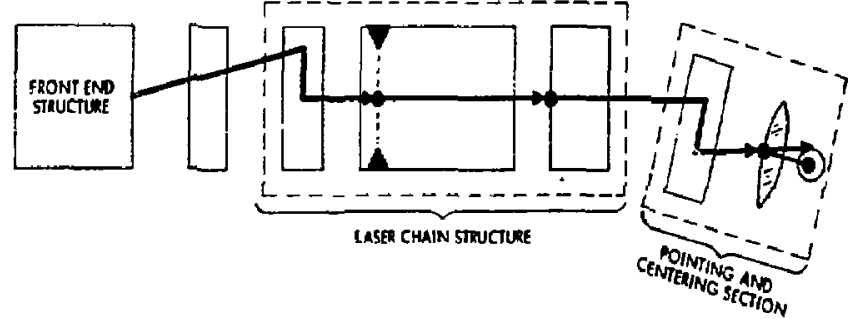

a

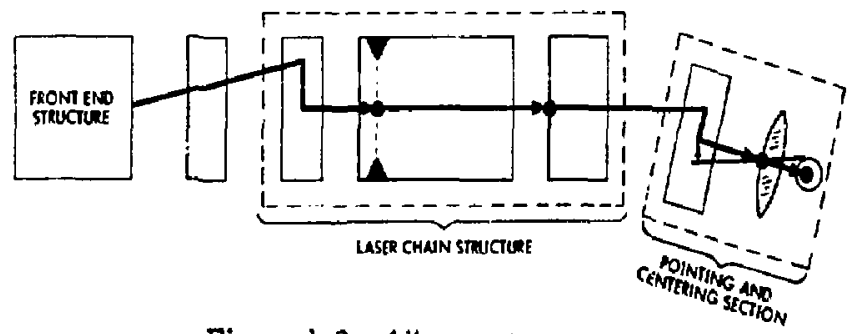

Figure 1-2. Alignment seqtence, 
2. The beam passes through the active elemests of the laser chain and strikes the entrance aperture of the final spatial filter off center. As shown in Figure 1-2, the laser che in FEP turns the beam in angle until it is centered on the entrance aperture of the spatial filter.

3. Turning the beam in angle as described above may, depending on the specific separations of the particular laser chain involved, upset the previously established alignment of beam centering on the apodized aperture. If SD, an iterative process continucs until both alignments are achieved simultaneously.

4. The beam passes through the final spatial filter. The pointing and centering mirrors now act in unison, (providing translation and beam pointing as shown in Figures 1-2d and 1-2e to make the beam pass through the center of the final focusing lens and at an angle which will cause the focused spot to fall at the correct position at the surrogate target.

Two particularly attractive characteristics have been achieved with this system which should be pointed out here:

1. If any one beam splitter path is misaligned, that laser chain can correct for it with its own FEP and without upsetting the alignment of any other laser chain.

2. If all of the laser chains abruptly lose alignment, the implication is that the front end structure has changed. Any one laser chain can then be brought into alignment using the front end structure's $\overline{F E P}$. This then, will bring all chains back into alignment.

\section{ALIGNMENT SYSTEM COMPONENTS}

A srief description of each device along with a description of the role it plays in the system is now presented. Detailed descriptions and performance antlyses appear in the foliowing Sections. 


\section{Pointing and Focusing Sensor (P/F)}

This sensor detects lateral displacement (pointing) errors of the focused spot relative to the surrogate target with a simple quad detector. Its pointing reference is derived from a retroreflected portion of the alignment beam.

The sensor also detects focus errors with a single detector which is optically commutated from a position in front of the plane of focus to a position behind the plane of focus. The sensor utilizes the alignment laser beam which has been refiected by the surrogate target and passes through the last reflecting polarizer.

The combining of these two functions is cost effective and necessary since the pointing and focusing detectors must be precisely boresighted to one another.

\section{Final Lens Centering Sensor (FLC)}

This sensor datermines the position of the alignment laser beam on the final focusing leas, It consists of four small detector assemblies which index into the outer edge of the final incusing lens. To enhance performance, the alignment beam edge is sharpiy truncated by an apertere stop which is indexed into the beam ju it after the pin hole.

Apodized Aperture Centering Sensor (AAC)

This sensor determines the position of the center of the beam with respect to the center of the apooized aperture. It consists of an objective lens and a quad detector which is located in the plane of focus of the apodized aperture. The beam from the pinhole, which is insident upon the apodized aperture, also utilizes the truncated beam during this alignment.

This sensor views the apodized aperture via reflection from the polarizer which follows it in the amplifier chain.

\section{Pinhole Sensor (P/H)}

This sensor is used to align the master oscillator beam and the alignment laser beam to the pinhole assembly in angle and translation. In accomplishing this, the two beams are aligned to one another in translation and angle. It is qualified at assembly in angle to the pinhole and in translation to the lens that precedes the pinhole. 


\section{Two Axis Mirror Gimbal}

Two mirrors are used at the end of each amplifier chain to direct the beam through the final focusing lens to the target. In the proposed alignment system, each of these mirrors is gimballed about two axes which are orthogonal to one another and parallel to the mirror surface.

The mirror gimbals feature fine and coarse steps and can be actuated remotely with stepper motors which are a part of the assembly, or they can be manually operated.

\section{Front End Positioner (iEP)}

The requirement to translate and/or turn the seam in angle occurs at a number of points in the Shiva alignment system. Most of these points are located sear the beginning of the amplifier chains where precision and accuracy of motion is much less siringent than the target. A single, universal device has been designed to satisfy these requirements. This device is the FEP.

Ry translating a corner reflector array of three mirrors in two orthogonal directions the beam can be made to translate in $X$ and $Y$. A fourth mirror is gimbaled about two axes for beam pointing.

Aperture Indexing Device (AID)

The aperture indexing device is locuted after the pinhole and is used to truncate the beam profile to enhance the performance of certain sensors. Either one of two aperture stops can be indexed into the beam with great centering precision. One stop creates a very small diameter beam $(2 \mathrm{~mm}$ at the pinhole or $14 \mathrm{~mm}$ at the laser chain centering sensor) and the second stop clips the edges of the beam to provide a sharper radiant discontinuity than that provided by the apodized aperture.

\section{Laser Chain Cente ring Sensor}

This sensor is similar to the AAC sensor. It senses the position of the laser beam incident on the polarizer located just before the final spatial filter. The same aperture stop is used to vignette the beam that is used with the AAC. 
Aligament Laser

This $1.06 \mu \mathrm{m}$ laser is aligned with the master oscillator and is then used as the energy source to provide all alignments and path length equalization.

Buring path length equalization, its output is mode locked and provides 0.5 watts average power. During alignment the average output power is 1.6 watts.

Mode-locked pulse duration is 200 psec.

\section{Master Console}

The master console provicles controls that enable remote manual and automatic adjustments to be made. In acidition, $i$ contains status indicators and anaiog displays that show the current alignment status of the various alignment subsystems. Sensor powe; shiter indexing and the aperture stop a re controlled from this unit. Also contained within the console is the control electronics for the stepping motors that control the centering, pointing and focusing of the beam. The console alsc receives commands and exchanges status information with the Livermore computer.

\section{Portable Test Box}

The portable test box is used for providing localized sensor information for the manually adiusted centering, pointing, and path length mechanisms. It consists of analog zero center meters, with range sensitivity switching. It requires no external power or battery source. The maters are diriven directly by the buffered sensor signals.

\section{ALIGNMENT SYSTEM FEATURES}

A number of attractive functional characteristics have been realized in tinis alignment system which are not immediately apparent from the preceding ove rview descriptions.

\section{Sensor Placement}

The pointing and focusing scnsor placement and the focal conditioning at the surrogate target have been selected in a way that minimizes the sensors sensitivity to movement. 


\section{Sensors Accommodation to Changes in Shiva Configuration}

Detailed optical analyses have been performed which demonstrate the primary, pointing and focusing sensor's ability to tolerate reasonable wave front distortions in the Shiva amplifier. Should the se distortions exceed expectations because of a change in Shiva design, they can be easily and inexpensively accommodated by adding correction to the serour objective lens,

\section{Growth Potential}

Ali actuating mechanisms provide the option of manual, remote/manual or automatic operation. The thought here is that a hasic mechaism can be initially procured in its least expensive form (manuaii). If operational experience later mandates remote/manual or automatic operation, motor drives car be procured and easily installed.

\section{Display and Control}

For a $20 \mathrm{arm}$ Shiva there are about 200 alignment conditions which rnust be monitored. From the standpoint of human engineering as well as cost it is important that the display of such a plethora of information be simplifiec. The Hughes approach to the problem is to provide a go, no-go status indication for each chain.

If the status of any single chain is indicated to be no-go, then an analogue disciay of all of its alignment parameters can be operator selected. The selected channel's adjustments are also made available to the set of controls on the panel. The total status irformation is continuously available to the Shiva master computer. 


\section{SYSTEM DESCRIPTION}

\section{SYSTEM PERFORMANCE REQUIRENENTS}

\section{Performance Specifications}

\section{Alignment Accuracy}

The Shiva alignment accuracy specifications, as proviried by livermore Laboratory, are given in Table 2-1. The master oscillator (MO) to pinhole speciaccations were given indirectly as follows:

1. The MO beam has gaussian intensity with 99 percent of energy within $20 \mathrm{~mm}$ diémeter.

2. The pishole diameter will pass 50 percent of the forused energy

3. The aiignment will be such that no less than 45 percent is transmitted

The centering requirements at all specified apertures shall be \pm 1 percent of the diameter. The remaining specifications were given directly as tabulated.

The basic functions that are required of the alignment system are enumerated in Table 2-2. The socond columr indicates the number of axes for each function. Tables $2-3$ through $2-9$ tabulate the exror allocation fo: performing each of these functions. The errors may be considerably smaller than the allocation.

Table 2-1. Alignment accuracy specifications

MO beam centered on pinhole assembly input lens

MO focused spot centered on pinhole

Beam center ed through apodized aperture

Beam centered at end of laser chain

Beam centered in focusing lens

Focused spot on target, trangverse

Focused spot on target and focus offset

by $2 \mathrm{fam}$

Path length matching $\pm 0.2 \mathrm{~mm}$

$\pm 2 \mu \mathrm{m}$

$\pm 0.2 \mathrm{mn}:$

$\pm 1.4 \mathrm{~mm}$

\pm 2 nim

$\pm 2 \mu \mathrm{rn}$

$\pm 7 \mu \mathrm{m}$

$\pm 3 \mathrm{~mm}$ 
Table 2-2, Functions required of alignment system

\begin{tabular}{|l|c|}
\hline \multicolumn{1}{|c|}{ Alignment function } & Number of Axes \\
\hline 1. Master oscillator to input lens of pinhole assembly & 2 \\
2. Maste: oscillator focused spot to pinhole & 2 \\
3. Alignment laser to input lens of pinhole assembly & 2 \\
4. Alignment laser focused spot to pinhole & 2 \\
5. Centering of beam into apodized aperture & 2 \\
6. Centering of beam at end of laser chain & 2 \\
7. Front end assembly to laser chains, angle & 2 \\
8. Front end assembly to laser chains, transverse & 2 \\
9. Centering of beam at target chamber & 2 \\
10. Pointing locused spot at surrogate target & 2 \\
11. Focusing spot at surrogate target & 1 \\
12. Offset focusing & 1 \\
13. Path length matching between laser chains & 1 \\
\hline
\end{tabular}

Table 2-3. Pinhole lens centering error allucation

\begin{tabular}{|lc|}
\hline Serisor alignment & $115 \mu \mathrm{m}$ \\
Align laser or MO & \\
FEP, transverse mode & $115 \mu \mathrm{m}$ \\
Drift & $115 \mu \mathrm{m}$ \\
\hline RSS total & $200 \mu \mathrm{m}$ \\
\hline
\end{tabular}

Table 2-4. Facal point to pinhole error allocation

\begin{tabular}{|c|c|c|}
\hline Sensor & $1.4 \mu \mathrm{m}$ & $7 \mu \mathrm{rad}$ \\
\hline $\left.\begin{array}{l}\text { Align laser or MO } \\
\mathrm{FEP} \text {, angular mode }\end{array}\right\}$ & $\perp \mu \mathrm{m}$ & $5 \mu \mathrm{rad}$ \\
\hline Drift & $1 \mu \mathrm{m}$ & $5 \mu \mathrm{racl}$ \\
\hline RSS total & $2 \mu \mathrm{m}$ & $10 \mu \mathrm{rad}$ \\
\hline
\end{tabular}


Table 2-5. Pointing error allocation

\begin{tabular}{|ll|}
\hline Sensor angular alignment & $0.3 \mu \mathrm{m}$ \\
Sensor transverse alignment & $0.3 \mu \mathrm{m}$ \\
Control system ACC $(5 \mathrm{ES} \times 2)$ & $0.45 \mu \mathrm{m}$ \\
Offset focus & \\
Focus mechanism error & $1.3 \mu \mathrm{m}$ \\
Beam centering error & $1.3 \mu \mathrm{m}$ \\
100 sec drift (all optical components in chain) & $0.5 \mu \mathrm{m}$ \\
\hline RSS total & $2.0 \mu \mathrm{m}$ \\
\hline
\end{tabular}

Table 2-b. Centering error allocation

\begin{tabular}{|c|c|}
\hline Sensor noise & $20 \mu \mathrm{m}$ \\
\hline Sensor alignment and balance & $12 c_{\mu \mathrm{m}}$ \\
\hline Control system accuracy & $120 \mu \mathrm{m}$ \\
\hline MO to AL center ing & NA \\
\hline $\begin{array}{l}\text { AA centering } \\
\text { Inde: aperture match }\}(18 \mathrm{~mm} \text { beam to } A A)\end{array}$ & $140 \mu \mathrm{m}$ \\
\hline Drift & $50 \mu \mathrm{m}$ \\
\hline RSS total & $230 \mu \mathrm{m}$ \\
\hline
\end{tabular}

Table 2-7, Last spatial filter centering error allocation

\begin{tabular}{|ll|} 
Sensor alignment error & $0.70 \mathrm{~mm}$ \\
Index aperture repeatability & $0.05 \mathrm{~mm}$ \\
Beam splitter FEP & $0.70 \mathrm{~mm}$ \\
Chain FEP & $0.70 \mathrm{~mm}$ \\
Drift & $0.70 \mathrm{~mm}$ \\
\hline RSE total & $1.4 \mathrm{~mm}$ \\
\hline
\end{tabular}


Table 2-8. Apodizud aperture centering error allocation

\begin{tabular}{|lr|}
\hline Sensor alignment (14 mm lo AA) & $10 \mu \mathrm{m}$ \\
Index aperture repeatability & $7 \mu \mathrm{m}$ \\
Beam splitter FEP & $10 \mu \mathrm{m}$ \\
Chain FEP & $10 \mu \mathrm{m}$ \\
Drift & $7 \mu \mathrm{m}$ \\
\hline RSS total & $20 \mu \mathrm{m}$ \\
\hline
\end{tabular}

Table 2-9. Path length error allocation

\begin{tabular}{|l|}
\hline Laser stability \\
Sensor/signal processing \\
Mech adjustment \\
\hline RSS total \\
\hline
\end{tabular}

Alignment of the MO and alignment lasers to the pinhole lens and the pinhole itself requires two axis transverse and two axis angular contrcl of the two lasers independently. They are combined in a beamsplitter at the entrance to the pinhole assembly where a centering and pointing renso: is calibrated to the pinhole assembly. Sensor alignment error represents the calibration accuracy and stability of the sensor and structura! integrity between it and the pinhole structure. Drift has been included in all tables on the belief that all alignments are continually in motion and the drift allocation is merely to reflect that fact and indicate the magnitude of drift that is acceptable. Since alignment of the focused spot to the pinhole is accomplished with an angular control, the error is also given in angular units, based on an $\mathrm{f} / 10$ pinhole lens.

Pointing at the surrogate target is addressed next as the accuracy requirements impacts several other alignments. The sensor is allocated $0.3 \mu \mathrm{m}$ due to angular aligument of the sensor $(19 \mu \mathrm{rad}$ at the sensor for baseline design) and $0.3 \mu \mathrm{m}$ due to transverse alignment $(225 \mu \mathrm{m}$ at the sensor for the baseline design). The control system has been allocated $0.45 \mu \mathrm{m}$ to match the gimbal fine step eize of $0.2 \mu \mathrm{rad}, \quad(0,45 \mu \mathrm{m}$ represents two resolution elements.) The lion's share of pointing error bas been allocated to the offset 
focus operation since the focus sensor is not functioning. The focus mechanism has been allocated $1.3 \mu \mathrm{m}$ to account for lateral motion while moving over an axial distance of $2 \mathrm{~mm}$. This implies a very high degree of mechanical stability. Another $1,3 \mu \mathrm{m}$ has been allocated to the effect of beam centering error on the less when the lens is moved. Figure 2-1 shows how this occurs. If there is a centering error, $\Delta_{\mathrm{X}}$, at the lens, and the lens is moved the distance $\Delta_{Z}$, to the desired focal plane, then the center of the spot on the target will have moved transversely by $\Delta \mathrm{X}_{\mathrm{T}}$. The error, $\Delta \mathrm{X}_{\mathrm{T}}$ is given by:

$$
\Delta \mathrm{X}_{\mathrm{I}}=\Delta_{\mathrm{X}_{\mathrm{I}_{1}}} \cdot \frac{\Delta \mathrm{Z}}{\mathrm{F}}
$$

When the focal plane motion is the maximum, $2 \mathrm{~mm}$, and $1.3 \mu \mathrm{m}$ shift is allowed, then the beam centering error must be less than $230 \mu \mathrm{m}$. This turns out to be an order of magnitude smaller than the original centering specification*.

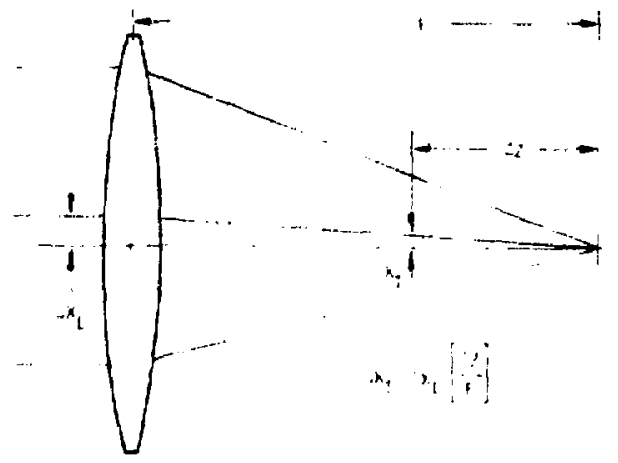

Figure 2-1. Pointing error caused by centering error and offset focusing

\footnotetext{
*Perhaps the pointing accuracy can be loosened for large targets?
} 
Tabie $2-6$ shows the centering error allocation to obtain the $230 \mu \mathrm{m}$ needed to keep the pointing error within specifications. Note that the alignment of the truncated reference beam to the apodized aperture is a factor in centering since the centering control system utilizes the truncated reference for alignment. This is not desirable but as yet no other method for a centering reference has been generated.

Tables 2-7 and 2-8 show both the beam splitter FEP and the chain FEP resolution as error contributions. This is so because they effectively are in series. The apodized aperture centering error specification is also on order of magnitude tighter than the given specification because of the offset focus and centering interaction. The remainder of the Tables 2-7 through 2-9 are self explanatory.

\section{Component Specification}

Table 2-10 gives the control requirements for the laser to pinhole assembly alignment and relates them to the mechanical motion required by the control mechanism. The beam motion is twice the transverse or angular motion of the mechanism therefore when total beam motion is converted to single

Table 2-10. Control mechanism (FEP) requirements for laser to pirhole alignment

\begin{tabular}{|l|c|c|}
\hline $\begin{array}{c}\text { Transverse motion at } \\
\text { pinhole lens }\end{array}$ & $\begin{array}{c}\text { Beam motion } \\
\text { total two axes }\end{array}$ & $\begin{array}{c}\text { Mechanical } \\
\text { motion per axis }\end{array}$ \\
\hline Azcuracy & $\pm 100 \mu \mathrm{m}$ & $\pm 36 \mu \mathrm{m}$ \\
Range & $4 \mu \mathrm{mm}$ & $1.4 \mathrm{~mm}$ \\
Resolution & $50 \mu \mathrm{m}$ & $18 \mu \mathrm{m}$ \\
Prealignment range & $10 \mathrm{~mm}$ & $3.6 \mathrm{~mm}$ \\
Prealignment resolution & $0.5 \mathrm{~mm}$ & $180 \mu \mathrm{m}$ \\
Transverse motion of & & \\
$\quad$ focal point & & \\
Accuracy & $\pm 1 \mu \mathrm{m}$ & $\pm 1.8 \mu \mathrm{rad}$ \\
Range & $500 \mu \mathrm{m}$ & $0.9 \mathrm{mrad}$ \\
Resolution & $0.5 \mu \mathrm{m}$ & $0.9 \mu \mathrm{m}$ \\
Prealignment range & $5 \mathrm{~mm}$ & $9 \mathrm{mrad}$ \\
Prealignment resolution & $100 \mu \mathrm{m}$ & $180 \mu \mathrm{rad}$ \\
\hline
\end{tabular}


axis mechanism the conversion is $1 /(2 \sqrt{2})$. I'realignment on the table is to indicate the range and resolution of the wrench-type adjustrent that must be provided for prealignment of the mechanism when it is first installed. It is important that the mechanism can easily be prealigned to well within the normal operating range. Table $2-11$ gives the same data for the same alignment mechanism used to aiign toth ends of the laser chain. I he data is moditied by the geometry since the mechanism is between $0.3 \mathrm{~m}$ anc $10 \mathrm{~m}$ from the first aperture. The additional requirements have been folded into the mechanism specification. Table 2-12 presents pathlength control parameters. The requirements for all of the control mechanisms have been combineo in Table 2-13 to provide a specification for a

Iable 2-11. Control mechanism and (FEF) requirements for laser chain alignment

\begin{tabular}{|c|c|c|c|}
\hline $\begin{array}{l}\text { Transverse motion al } \\
\text { apodized aperalure }\end{array}$ & $\begin{array}{l}\text { Beam motion } \\
\text { total two axis }\end{array}$ & \multicolumn{2}{|c|}{$\begin{array}{c}\text { Mechanical motion } \\
\text { per axis }\end{array}$} \\
\hline $\begin{array}{l}\text { Accuracy } \\
\text { Range } \\
\text { Resolution } \\
\text { Prealignment range } \\
\text { Prealignment resolution }\end{array}$ & $\begin{array}{l} \pm 10 \mu \mathrm{m} \\
10 \mathrm{~mm} \\
5 \mu \mathrm{m} \\
5 \mathrm{~cm} \\
1 \mathrm{~mm}\end{array}$ & \multicolumn{2}{|c|}{$\begin{array}{l} \pm 3.6 \mu \mathrm{m} \\
3.5 \mathrm{~mm} \mathrm{i+7mm} \text { (2) } \\
1.8 \mu \mathrm{m} \\
17.5 \mathrm{~mm}(+4 \mathrm{~mm}) 2 \\
350 \mu \mathrm{m}\end{array}$} \\
\hline $\begin{array}{c}\text { Transverse motion at } \\
\text { last spatial } \\
\text { Filter (2) }\end{array}$ & & $\begin{array}{l}\text { Mech angular } \\
\text { motion per } \\
\text { axis }\end{array}$ & $\begin{array}{l}\text { Motion at } \\
\text { AA }\end{array}$ \\
\hline $\begin{array}{l}\text { Accuracy } \\
\text { Range } \\
\text { Resolution } \\
\text { Prealignment } r \text { ige } \\
\text { Prealignment resolution } \\
\text { Acquisition range }\end{array}$ & $\begin{array}{l} \pm 0.7 \mathrm{~nm} \\
20 \mathrm{~mm} \\
350 \mu \mathrm{m} \\
40 \mathrm{~mm} \\
4 \mathrm{~mm}\end{array}$ & $\begin{array}{l}25 \mu \mathrm{rad} \\
790 \mu \mathrm{rad} \\
12 \mu \mathrm{rad} \\
1.4 \mathrm{mrad} \\
140 \mu \mathrm{rad}\end{array}$ & $\begin{array}{l}(7 \mathrm{~mm}) \\
(14 \mathrm{~mm})\end{array}$ \\
\hline
\end{tabular}

(1) Distance from FEP to apudized aperture varies from 0.3 to $10 \mathrm{~m}$

(2) Correction at spatial filter introduces motion at apodized aperture

(3) $0.1 \mathrm{mrad}$ at apodized aperture causes $1 \mathrm{~mm}$ at spatial $f$ iter. 
Tabie 2-12. Path length alignment

\begin{tabular}{|l|c|c|}
\hline & Optical length & Mechanical motion \\
\hline Accuracy & $\pm 3 \mathrm{~mm}$ & $\pm 1.5 \mathrm{~mm}$ \\
Range & $3 \mathrm{~cm}$ & $1.5 \mathrm{~cm}$ \\
Resolution & $1.5 \mathrm{~mm}$ & $0.7 \mathrm{~mm}$ \\
Acquisition range & $3 \mathrm{~cm}$ & $1.5 \mathrm{~cm}$ \\
\hline
\end{tabular}

Table 2-13. Front end alignment (FEP) specification

Iransverse motion

Acciracy

$\pm 3.6 \mu \mathrm{m}$

Range

$11 \mathrm{~mm}$

Resolution

$1.8 \mu \mathrm{m}$

Prealignment range

$32 \mathrm{~mm}$

Prealignment resolution

$180 \mu \mathrm{m}$

Angular motion

Accuracy

Range

Resolution

Prealignment range

$\pm 1.8 \mu \mathrm{rad}$

Prealignment resolution

$900 \mu \mathrm{rad}$

$0.9 \mu \mathrm{rad}$

$9 \mathrm{mrad}$

$140 \mu \mathrm{rad}$

Axial motion

Accuracy

$\pm 1.5 \mathrm{~mm}$

Range

$1,5 \mathrm{~cm}$

Resolution

$0.7 \mathrm{~mm}$

Prealignment range

Prealignment resolution

E EP that will fulfiil all requirements and can be used interchangeably in any location.

The requirements for the centering and pointing nirror are presented in Table 2-14. The first column gives the system requirement and the 
Table 2-14. Control mechanism requir ements for the centering and pointing mirrors $(0.395 \mathrm{~m}$ focal distance)

\begin{tabular}{|c|c|c|c|}
\hline Pointing & At target (total) & \multicolumn{2}{|c|}{ Mirror angle (per axis) } \\
\hline Accuracy & $\pm 0.45 \mu \mathrm{m}$ & \multicolumn{2}{|c|}{ $\pm 0.4 \mu \mathrm{rad}$} \\
\hline Active range & $\pm 2 \mathrm{~mm}$ & \multicolumn{2}{|c|}{ $\pm 2.7 \mathrm{mrad}$} \\
\hline Active resolution & $<0.22 \mu \mathrm{m}$ & \multicolumn{2}{|c|}{$<0.2 \mu \mathrm{rad}$} \\
\hline Prealignment range & $\pm 20 \mathrm{~mm}$ & \multicolumn{2}{|c|}{ $\pm 27 \mathrm{mrad}$} \\
\hline Prealignment resolution & $\leq 0.2 \mathrm{~mm}$ & \multicolumn{2}{|c|}{$<1.9 \mathrm{mrad}$} \\
\hline . & & \multicolumn{2}{|c|}{ Mirror angles } \\
\hline Centering & Ai focus lens & $l=1.76 \mathrm{~m}$ & $=8.8 \mathrm{~m}$ \\
\hline Accuracy & $130 \mu \mathrm{m}$ & $\pm 26 \mu \mathrm{rad}$ & $\pm 5.2 \mu \mathrm{rad}$ \\
\hline Active range & $\pm 20 \mathrm{~mm}$ & $\pm 5.6 \mathrm{mrad}$ & $\pm 1.14 \mathrm{mrad}$ \\
\hline Active resolution & $65 \mu \mathrm{m}$ & $<13 \mu \mathrm{rad}$ & $<2.6 \mu \mathrm{rad}$ \\
\hline Prealignment range & $\pm 50 \mathrm{~nm}$ & $\pm 14 \mathrm{mraci}$ & $\pm 2.85 \mathrm{mrad}$ \\
\hline Prealignment resolution & $<2 \mathrm{~mm}$ & $<0.4 \mathrm{mrad}$ & $<80_{\mu \mathrm{rad}}$ \\
\hline
\end{tabular}

second column give the resulting mirsor gimbal requirement. For the centering mirror two sets of :equirements are given since the distance between the centering and pointing mir ror varies from $1.76 \mathrm{~m}$ to $8.8 \mathrm{~m}$. Table 2-15 gives the combined requirements. In actual use the requirements will be between these two limits, and in fact each chain will probably require a different coarse and fine step size, depending on the distance between mirror and the geometry of the input and output line of sight. The requirements only indicate the range of parameters that will be required. (It should be noted that the baseline gimbal design is amenable to various step sizes and can be changed casily.)

Table 2-16 summarizes the environnental conditions of the liboratory environment. 
Table 2-15. Mirror gimbal requirements

\section{- Angular requirements}

Active range

Coarse $\quad \pm 9 \mathrm{mrads}$

Fine $\quad>20 \mu$ rads

Active resolution

Coarse

2.6 to $13 \mu \mathrm{rads}$

Fine

$<0.2 \mu \mathrm{rads}$

Prealign range (1)

$\pm 40 \mathrm{mrads}$

Prealign resolution

$<80 \mu \mathrm{rads}$

(1) Prealignment is a wrench-type adjustment

- Mechanical requirements

Resonal frequency

$>10 \mathrm{~Hz}$

Mechanical damping factor

$>0.1$

Table 2-16. Laboratory environmental corditions

- Temperature

$24^{\circ} \mathrm{C}$ nominal

$\pm 5.5^{\circ}$ cyclic variation (approximately 15 minute period)

$1.0^{\circ} \mathrm{C}$ maximum ceiling to 0 loor differential

- Airflow

0.4 meters/sec vertiral velocity

Air change every 30 seconds

- Vibration

Resonant peaks at

$$
\begin{aligned}
& \approx 16 \mathrm{~Hz}\left(5.6 \times 10^{-6} \mathrm{G}^{\prime} s\right) \\
& \approx 23 \mathrm{~Hz}\left(4.5 \times 10^{-6} \mathrm{G}^{\prime} s\right)
\end{aligned}
$$




\section{Design Constraints}

Beyond the system specifications; a group of design constraints or goals have evolved. In some instances these constraints have been self-imposed and in ohers they have been specified by Livermore Laboratory personnel. These constraints have driven the $\mathrm{L}_{\mathrm{s}}$ : whes system design and have been successfully realized in every case with tise exception of one.

- Because of the non-linear opticai effect and the resulting beam break-up, it is imperative that no additional glass be added in the high power beam by the alignment system.

- The entire alignment beam must be monitored by the focus and pointing sensor. Because of unavoidable wave-front distortions, samplang a small, untypical portion of the beam could lead to gross errors.

- Sensor position and orientation tolerances should, through optical leverage techniques, be made much less stringent than the focal point tolerances.

- All sensors should gain entry to the laser chain via an existing element, i.e., polarizers, and should not be required to index in and out of the high-power beam path. This is the goal which we have not realized in the case of sensing beam position on the final focusing lens. The difficulties and pitfalls in logic concerning this measurement are discussed in Appendix $\mathrm{H}$. The prcposed method does index small sensors into the outer edge of the beam while performing this alignment.

- The pointing and focusing sensor must be able to calibrate its pointing reference readily and in an absolute sense.

- It is recognized that the Shiva design will be undergoing modifications for some time to come. The alignment system chosen must be able to easily accommodate such changes. 
- The focus and pointing sensor must be able to acconmodate reasonable amounts of wave-front distortion. Hughes has chosen reasonable to mean one half wave of coma, one hall wave of spherical and one ball wave of random.

- Pointing mirror gimbals must be insensitive to orientation with respect to gravity.

- Because most cost elements are multiplied by 20 or 32 the number of Sniva arms) inexpensive, commercialiy availabie components should be given first consideration. This should not however, be done at the cost of reliability. For example, high roliability and relatively expensive switches would be the preferred choice. inexpensive, low-perfornance photodetectors would also be the preferred choice, because they do not compromise teliability and they also provice a method to improve performance if needed at a later date.

- The final reflecting polarizers which are in a rhomb configuration offer a means for bean translation (centering) as well as path length change. This approach has been abandoned because of possible degraviation in extinction coefficient and because of the desire not to mount these critical elements in pivoting assemblies.

- $\quad$ go status nust be ma intained with power off to actuators and sensols.

- Goo status for pointing and focusing must be maintaincti for a 100 second count down.

- Ar active alignment system was ruled unacceptable by Livernore Laboratory.

- All motor drives mist provide manual override.

- A distrust by Livermore Laboratory of rotating nechanisms in the beam lead to the abandonment of schemes which nutate the beam with retractable rotating wedges.

- The alignnent beam nusi be $1.06 \mu \mathrm{m}$ wavelength. 


\section{SYSTEM PHYSICAL/FLNCTIONAL DESCRIPTION}

This section discusses implementation of the alignment system functions. The functions are listed in Table 2-17. The control mechanism, the sensor, di:t the method of entry into the system are identified for each function. The individual components arc discussed in Sections 3 through 10, and are roierred to as required at pertinent points in other sections.

310 io Pinhole Lens Alignment

The beam from the NO must be centered on the input lens to the pishole assenbly. The components inolved isee left side of the simplitied functional schematic, Figure 2-A) are the MO, the MO FEP, the pinhole sensor and the piniole assembly. The FEP provides two axis steering of the bean:, wo axis irensverse motion of the beam, and optical path length change. The pinhole sensor has the capability fo: sensing both the transierse position and the input angle or a beam relative to its aperture.

The MO beam passes through the MO EEP to a beam splitter where a portion of the beam is reflected into the pinhole sensor. This arrangement is shown in Figure 2-3. The sensor provides a signal for the transverse control of the FEP. The pinhole scnsor and the ieamsplitter are rigidly mounted to the pinhole assembly and calibrated so that the beam is properly centered on the lens when the transverse sensor output signal is nulled.

\section{MO Focused Spot to Pinhole}

The focused energy from the MO must also be directed through the pinhole. The components involved are again the MO, the MO FEP, the pinhole sensor and the pinhole assembly. The energy reflected from the bcamsplitter for centering on the lens is also used to center the focused spot on the pinhole. The second function of the pinhole sensor is to measure angular error in two axes, and provide a signal for angular control of the FEP. Again the pinhoir sensor and the beamsplitter must be rigidly mounted to the pinhole assenbly and calibrated so that the spot is centered on the pinhole when the angle sensing part of the pinhole sensor is nulled. 
Table 2-17. Functions Required of Alignment System

\begin{tabular}{|l|l|}
\hline \multicolumn{1}{|c|}{ Alignment Function } & Number of Axes \\
\hline $\begin{array}{l}\text { 1. Master oscillator to input lens of } \\
\text { pinhole assembly }\end{array}$ & 2 \\
2. Master oscillater focused spot to pinhole \\
3. Alignment laser to input lens of pinhole \\
assembly
\end{tabular}

Alignment Laser (AL; to Pinhole Lens Alignment

Centering of the alignment laser beam on the pinhole lens is accomplished in the same way as the MO. The shutter that blocks the Alignment laser beam is opened and the one in front of the MO is closed. The combining beam splitter is snown in Figure 2-3.

\section{Alignment Laser Focused Spot to Pinhole}

The alifinment of the alignment laser to the pinhole is accomplished in the same way as alignment of thr MO. 


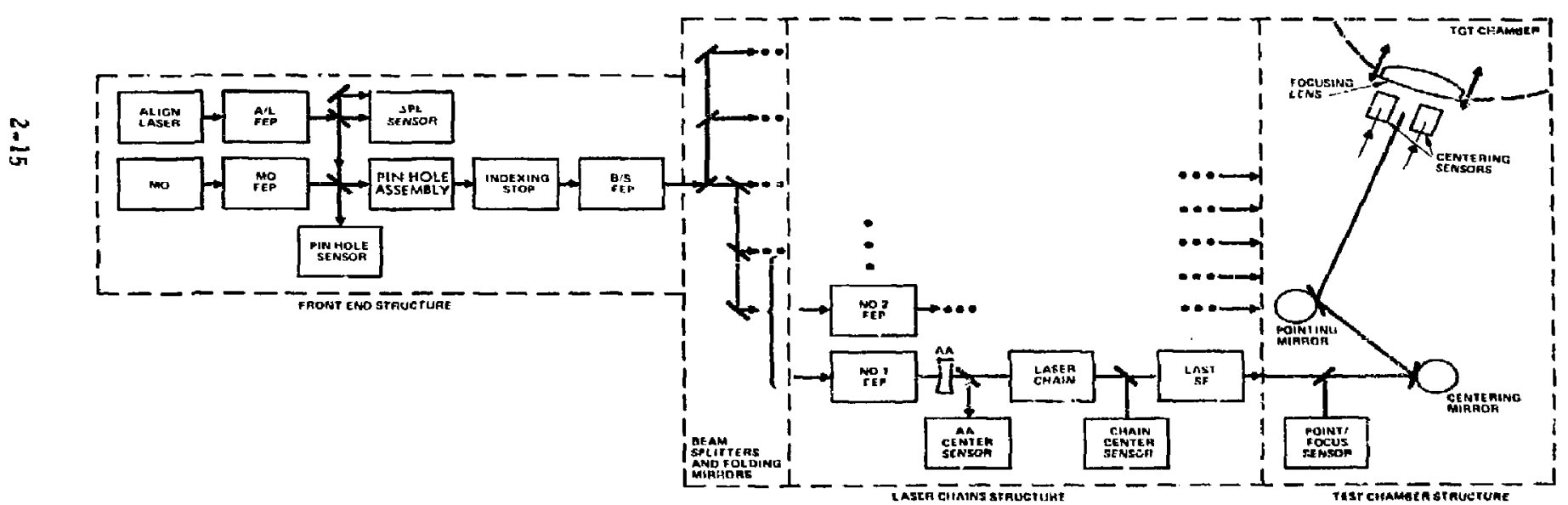

Figste 2.2. Simplified iurictional echematic of laser alignment control system 


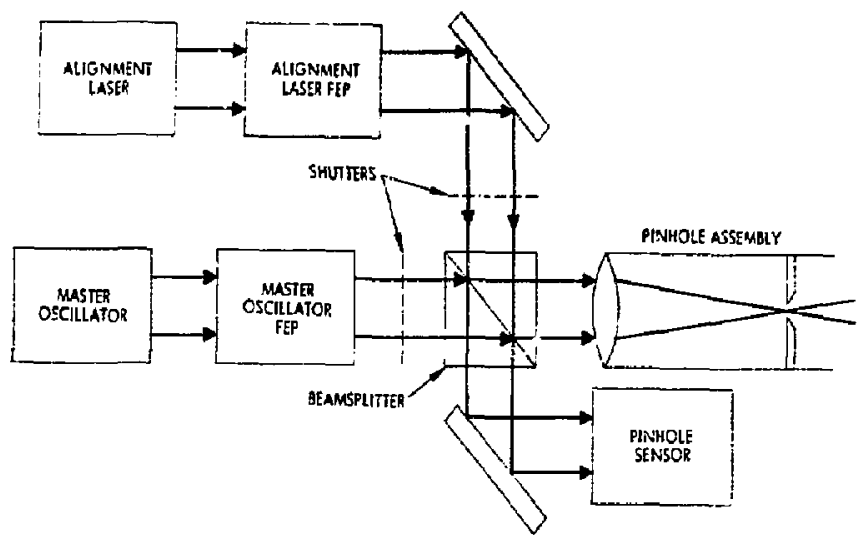

Figure 2-3. Master oscillator and alignment laser to pinhole alignment

\section{Indexing Stop}

The ind exing stop is a mechanism that indexes a $2 \mathrm{~mm}$ aperture stop, an $18 \mathrm{~mm}$ aperture stop or no stop into the $40 \mathrm{~mm}$ beam right after the output lens of the pinhole assembly. The stops are precisely centered with respect to the bram iy rigid mounting and calibration to the pinhole assembly. (See Figure 2-2.)

The indexing aperture is used to provide a $18 \mathrm{~mm}$ beam which is used in three places, (1) centering on the apodizing aperture (AA), (2) last spatial filter centering and (3) centering on the focusing lens.

If th: ir.put beam fuliy illuminates the apodizing aperture, then transverse motion of the input beam cannot be easily detected, because the inters ity profile is established by the aperture. However when the input beam is truncated at $18 \mathrm{~mm}$ then its motion can easily be detected afier the apodized aperture. This implementatic akes possible the use of the transmission polarizers as beam splitters since it allows sensing after the apodized aperture. 
When the $18 \mathrm{~mm}$ input beam reaches the last spatial filter, it has beet expandeo to $12.5 \mathrm{~cm}$. This is a converient size for transverse sensing at this point. Sensing the fuli $14 \mathrm{~cm}$, untruncated beam would require an aperture that is larger than $14 \mathrm{~cm}$.

The third advantage in utilizing the $18 \mathrm{~mm}$ aperture is for centering at the focusing lens. The truncation provides a well defined edge that allows for sensing the beam position at the target chamber.

The beam diameter into the pointing and focus sensor in the fine pointing mode is about $1 \mathrm{~cm}$. The $2 \mathrm{~mm}$ position of the indexing aperture provides a $2.1 \mathrm{~cm}$ at the sensor input that is used as a centering reference for the pointing and focus sensor. Alignment of the pointing and focus sensor will be discussed in detail in a following section.

Centering of Beam on Apodized Aperture

The beam must be centered on the apodized aperture of each laser chain. The components utilized are the FEP which is located at the sront end of each laser chain, the apodized aperture, the first transmission polarizer which is used as a beamsplitter, the apodized aperture centering sensor and the indexing aperture stop. (See Figure 2-4,) The $18 \mathrm{~mm}$ stop is used to provide a well defined $18 \mathrm{~mm}$ edge on the beam through the $20 \mathrm{~mm}$ apodizing

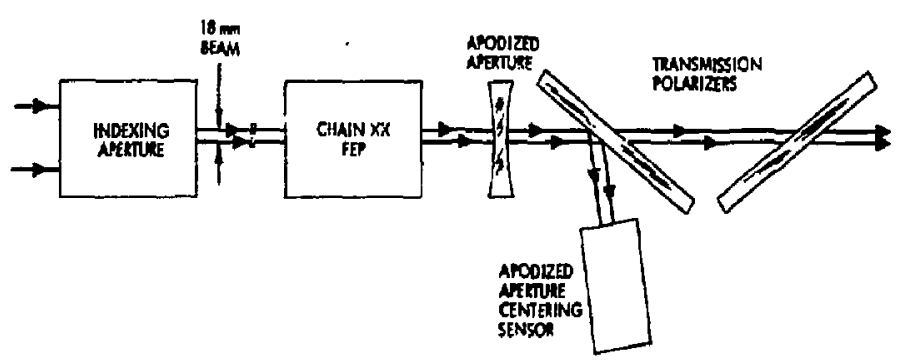

Figure 2-4. Apodized aperture centering implementation 
aperture. The apodized aperture sensor measures transverse motion of the $18 \mathrm{~mm}$ beam in two axes and provides a signal for the transverse control of the FEP. The transmission polarizer (that functions as a beamsplitter) and the apodized aperture sensor are rigidly mounted to the apodizing aperture and are calibrated so that the sensor is at null when the $18 \mathrm{~mm}$ beam and the $2 \mathrm{~mm}$ beam are preciseiy centered in the apodizing aperture.

Beam Centering at End of Laser Chain

The components utilized for centering the beam at the end of the laser chain are the indexing aperture, the FEP at the begianing of the laser chain, the seflecting polarizer at the end of the chain and the centering sensor. The indexing aperture is set for the $18 \mathrm{~mm}$ beam which is expanded through the chain to $12.5 \mathrm{~cm}$. This arrangement is shown in Figure 2-5. The transverse error signal from the sensor is utilized to control the angular capability of the FEP, which results in $1 \mathrm{~mm}$ of transverse motion at the end of the chain for $0.1 \mathrm{mrad}$ angular deviation at the input. The polarizer that is used as a beamsplitter and the centering sensor are mounted rigidly to the spatial filter and calibrated so that the beam is centered on the spatial filter when the sensor error is nulled.

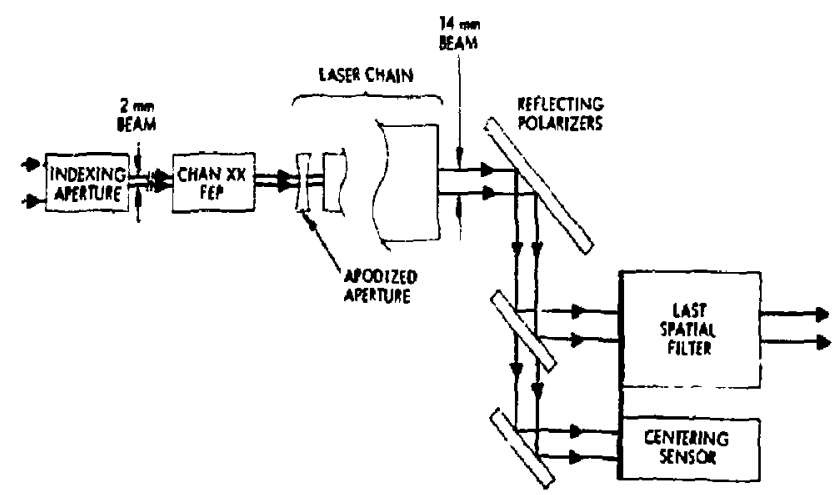

Figure 2-5. Laser chain centering implementation 


\section{Alignment of Front End Assembly to Laser Chain Structure}

The purpose of this alignment is to a lign the output beam s: om the pinhole assembly, transversely and angularly so that it enters all of the apodized apertures in the center and at the correct angle so that the beain will also be centered on all of the last spatial filters. This presumes that all of the laser chains are individually aligned so that if the beam traverses one of them properly it will be centered through all chains. This alignment is reeded so that movement of components before the indexing aperture can be corrected for without realigning all 20 (32) laser chains or if there is movement of the front end structure with respect to the laser chains structure. The alignment is accornplisined with the beam splitter FEP (See Figure 2-2) and the centering sensors (apodizing aperture and spatial filter) for any single chain.

\section{Control of FEP}

There is cross coupling between the transverse motion and the angular motion when adjusting either the FEP for the individual laser chains or the FEP that aligns the front end 10 all of the chains jointly. Purely transverse motion at the apodized aperture will cause transverse motion at the last spatial filter that is seven times larger (the beam expansion ratio from the apodized aperture to the last spatial filter is seven).

Angular motion introduced at the input for centering at the last spatial filter will also cause a tran. Lation at the apodized aperture. This translation is directly proportional to the distance from the FEP to the apodized aperture. For the FEPs that adjust the individual laser chains the distance varies from 0.3 meters to 10 meters. For the FEP that adjusts the input to the combined laser chains this distance varies from 9 meters to 23 meters. Where the FFP is closest to the apodized aperture the adjustment is reasonable simple since there is little effect on the apodized aperture centering when the last SF centering is adjusted. Probably one iteration will be adequate. However, where the distance is large, the operator will have to make incremental adjustments in order to converge to the correctly aligned condition. Tre step by step procedure is described in a later section. 
A simplified block diagram for a single axis/manual control is shown in Figure 2-6. It can be seen that for short distances between the apodized aperture and the FEP ( $\ell$ is small) the apodized aperture adjustment can be made and is not significantly effected by the other adjustment, but when the distance is large (say $l=10$ meters) then control must be applied in both angle ar:d translation in or der for the process to converge. Figure $2-7$ shows t.e correct compensation that must be incorporated. The idea of this compensation is to provide centering at the closest aperture (the apodized aperture) and for centering at the farthest aperture (the spatial filter) to ratate the beam about a pivot point that is effectively in the center of the first aperture. To accomplish this rotation the beam must translate and rotate at the FEP.

For purely manual control the compensation would be provided in a table or curves or for remote manual the compensation would be introduced elec tronically by driving both channels with the appropriate scale factor for the channel being adjusted. In either case the compensation shown would allow the two adjustments to be completely independent.

When the front end structure is being aligned to the laser chains structure with the remotely controlled beamsplitter FEP (See Figure $2-2$ ) the display to the operator will be from the apodi\%ed aperture centering stasor and last spatial filte; centering sensor from a single laser chain, however due to the sensitivity of the apodized aperture adjustment to the angular control, the operator must pay specific attention to the channels for which the apodized aperture is larthest from the beamsplitter FEP.

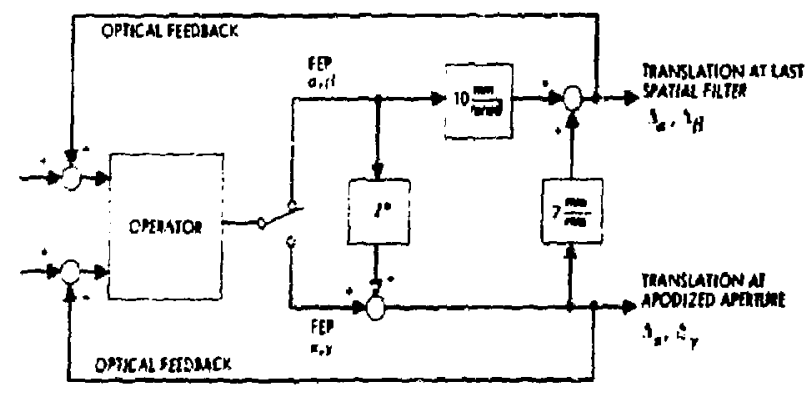

- IS THE CISTANCE FLOM FEV YO APODIZED AFERTURE

Figure 2-b. Manual control block diagram for apodized aperture and spatial filler centering 


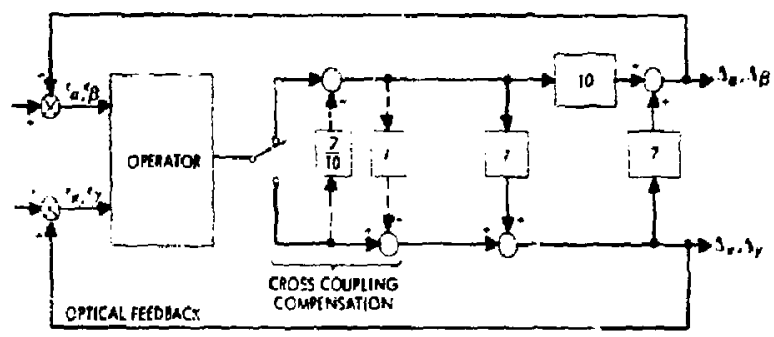

Figure 2-?, Remote control of apodizes aperture and last spatial filter centering with crosscoupling compensütion

\section{Centering At Target Chamber}

The components used in centering the beam on the $25 \mathrm{~cm}$ lens are the centering sensors shown (in Figure 2-8) on each side of and the turo gimbaled mirrors (pointing mirror and centering mirror). The indexing aperture is in the $18 \mathrm{~mm}$ position, which causes the beam at the lens to be truncated at 22 ci. diameter. The lour centering sensors are indexed into the edge of the truncated beam so that each sensor is approximately one-half illuminated by the truncated beam as shown in Figure $2-8$ and Figure $2-9$. The sensors are mechanically referenced to the lens so that when equal energy fails on opposing sensors, the beam is centered in the lens to the required precision. The mechanism is arranged so that tolerances are not stringent. This configuration (with the sensors at the edge of the beam) provides an acquisition field of $\pm 22 \mathrm{~cm}$, thereby insuring that the centerinf function will always know where it is.

Transverse control of the beam is implemented as shown in Figure 2-10. For simplicity the Figure 2-10 shows the single axis case where both mirrors are nominally at $45^{\circ}$ and they are operating in the same plane. It can be seen from the Figure that both mirrors must turn through the same angle in order to achieve pure transverse control with no angular motion, Transverse and angular control are diacussed later in this section. 

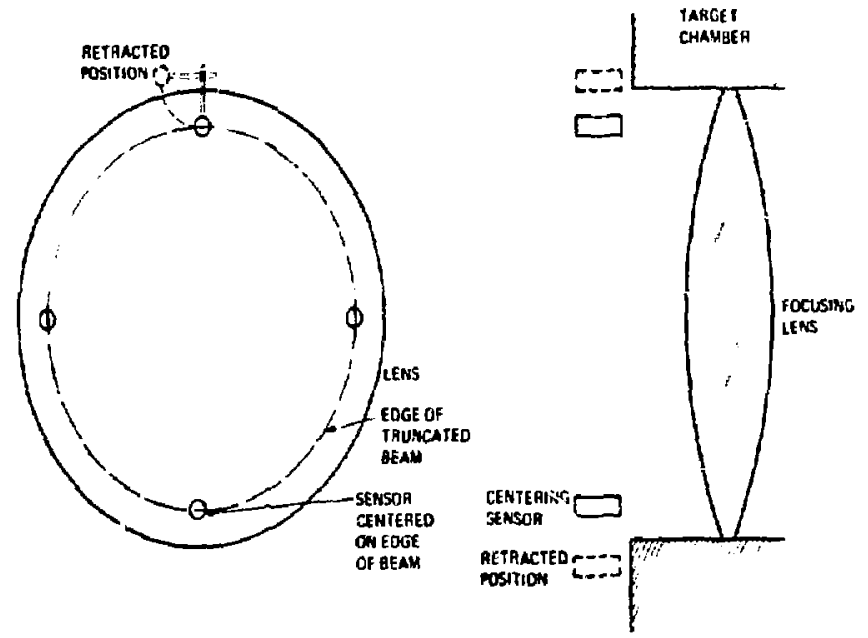

Figure 2-8. Location of centering sensors

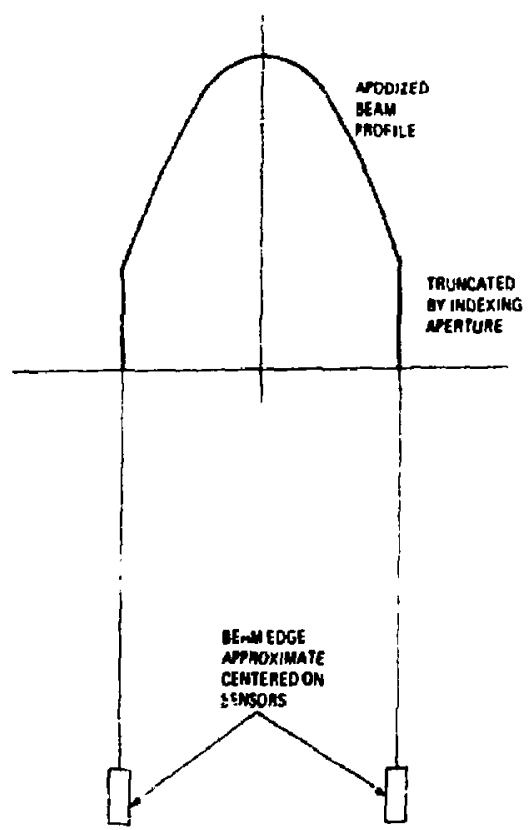

Figure 2-9. Centering sensor location in truncated beam profile 


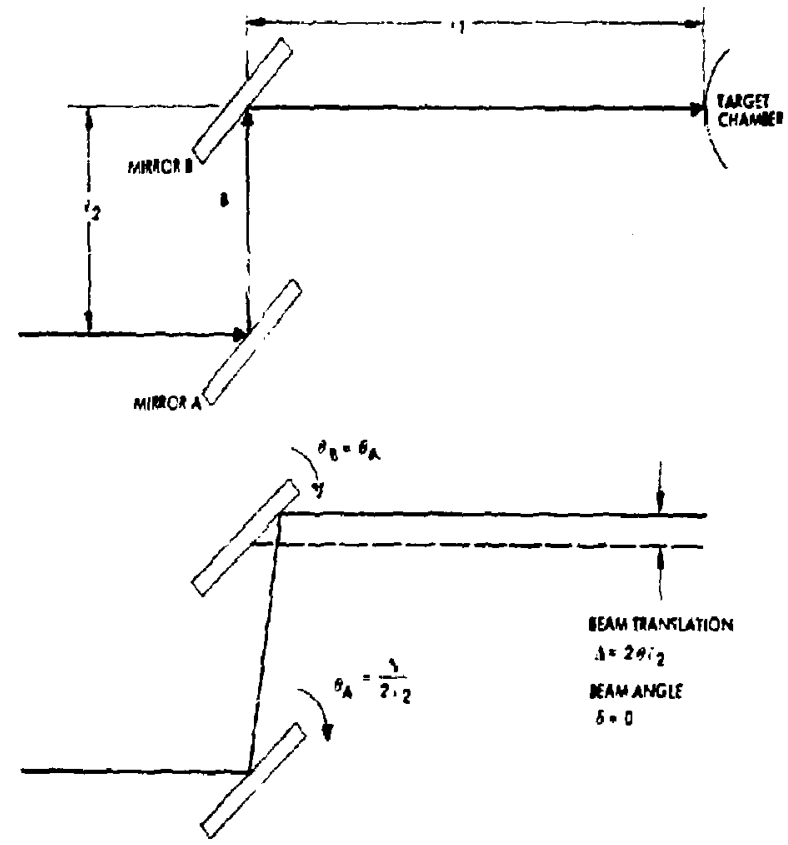

Figure 2-10. Control function for pure transverse control of beam

\section{Pointing at Sur rogate Target}

The components utilized for pointing at the surrogate target are the pointing and focus sensor, the retro-reflector, the last reflective polarizer which is used as a beam splitter and the two gimbaled mirrors. ILacation of the sensor in the optical train is shown in Figures $2-2$ and 2-11. The retro is used for referencing the sensor to the input beam from the laser chain. The vensor, beamsplitter (polarizer plate), and retro must be calibraled together and rigidly mounted together. Transverse nulling the sensor to the input beam requires that this whole assembly be translated as shown in Figure 2.11. Angular nulling requires that the sensor be rotated about an axis through the input lens.

Due to high accuracy (and hence resclution) that is required of the pointing function the field of view that can be obtained with this factor leads to a two stage pointing process which will be referred to as acquisition and fine pointing respectively.

Angular control or pointing of the beam, which moves the focused apot on the target, is implemented as shown in Figure 2-12. To illuatrate the 


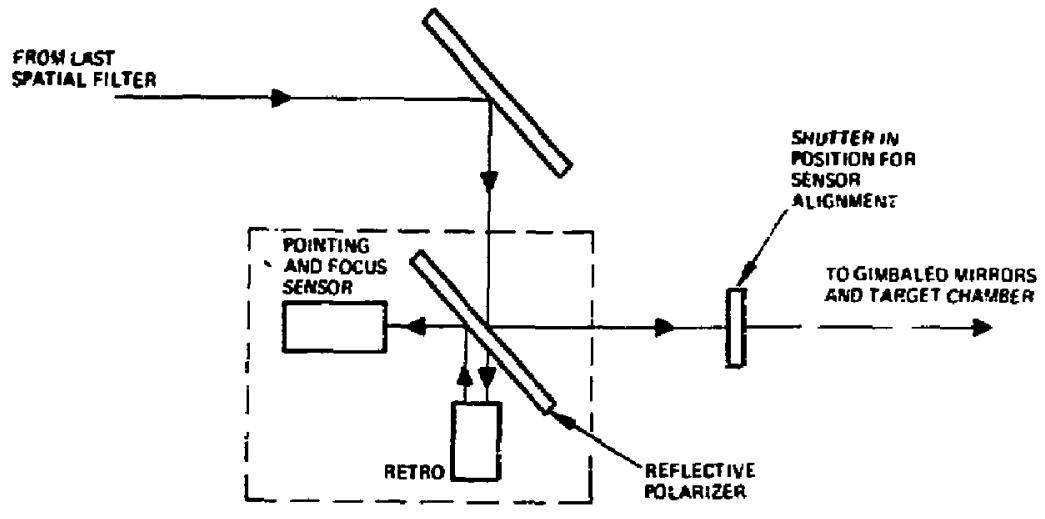

Figure 2-11. Location of pointing and focus sensor in optical train
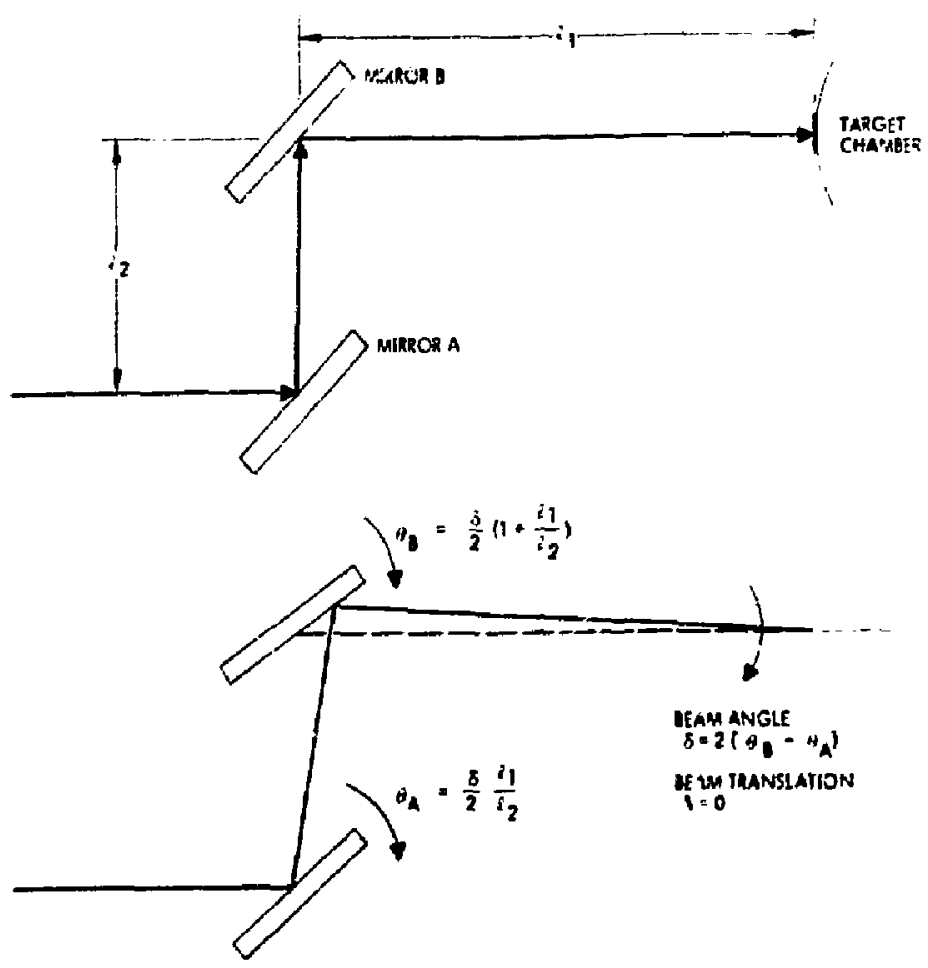

Figure 2-12. Control function for pure angular control of beam 
principle a simple single axis, planar case is shown where the mirrors are both nominally at $45^{\circ}$. It can be seen from Figure 2-12 that both mirrors must be operated to obtain angular control without causing transverse motion at the target chamber. It should be noted that the second mirror must always travel farther than the first. Control of the two mirrors is discussed next in this section.

Control of the Gimbaled Mirrors

Figures 2-10 and 2-12 show the relationship between the mirror angles and beam angular and transverse motion. There are swo basic approaches to control of pointing and centering function. One approach is to utilize mirro: $A$ as the centering control and mirror $B$ as the pointing control and clcse two separate servo loops (mirror A must be used for centering since it is the farthest away from the target chember). The two servos would operate independently, but the operation of one servo would cause an apparent error in the other that must be corrected. At first this appears the simplest solution. However, to prevent servo instability, one servo must be much slower than the other and this leads to very slow control of the joint functions.

A second approach is to treat the two mirrors as a combined control element and to drive both simultaneously so that the desired motion, angular or transverse, is achieved directly. For example if the centering error were zero and there were a pointing error of $\delta$, then mirror $A$ and mirror $B$ would be driven as follows:

$$
\begin{aligned}
& \operatorname{mirror} A \quad \theta_{A}=\frac{\delta}{2}(11 / 2) \\
& \text { mirror B } \theta_{B}=\frac{\delta}{2}(1+21 / Q 2)
\end{aligned}
$$

\section{(See Figures 2-10 and 2-12 for reference.)}

This will directly correct the pointing error and introduce no traneverte error. The transverse correction can be achicved in similar hashion. If the servo loop are closed this way, then two independent servos are reat ized with no cross coupling, although both gimbaled mirrors are driven for each function. Figure 2-13 shows a simplified block diagram for the servo. 


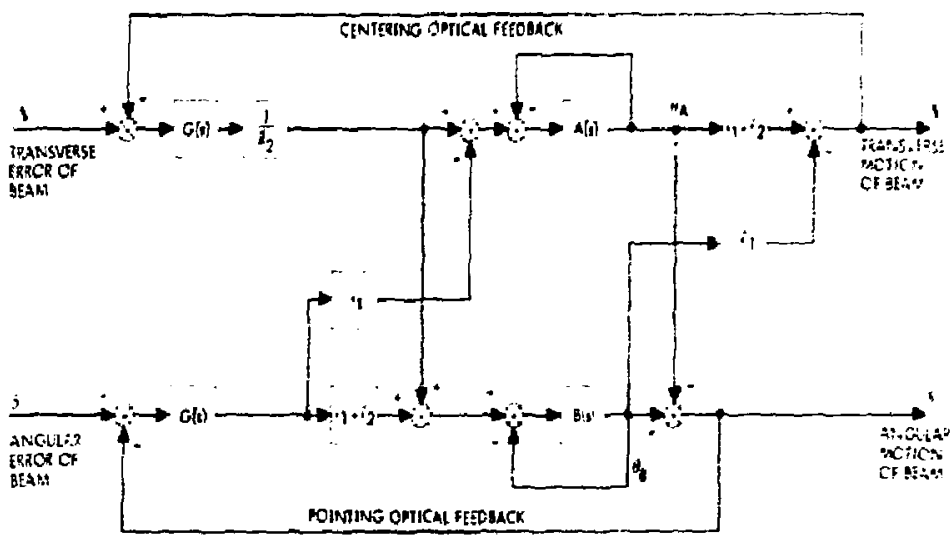

Figure 2-13. Pointing and cente:ing control implententation

For the actual sygtem where the mirrors are no: at $45^{\circ}$ and the output beam (from the two mirrors) is not in the same plane as the input beam, the electronic scale factor must take into account the geometry of the particular chains. The other cases were not worked out sirce the Shiva contiguration is not final at this writing. Since the proposed system incorporates stepping nirrors, either the number of steps or the step size lar both can be utilized to achieve the correct scaling. Another important factor is to insurc that the gimbaled axes of the two mirrors are as close as possiblc to being parallel. Although the servos could be coupled in such a way that nonparallel axes could be accommodated it would lead to unnceessary complication of the electronics. The nimbal axes of two mirrors can be made ;raliel by rotating oither mirror by rotating the basc. Essentially this is egurvalent to rotating about an axis that is orthogonal to both gimbal axes. The nisunt is similar to the comtion servo mourt used for rotating servo conponea: 3 , so that it can be solidly fastened down in any rotary posicion. The cuntris of two misrors to aflect centering and pointing is discussed in deiail in Appendix K. This appendix is comprised of sections from two prewous reports on a similar alignment system that is now being built. It shouln by noted that the equipment discussed in the appendix is more complex in that 
wideband dynamics were a primary concern, however the general approact to control and the effect of non-orthogonality of axes is pertinent.

The following factors have led to the implementation of pointing with a cuarse/fine ginbal drive in which the coarse pointing resolution is the same as the rentering resolution.

1. Angular resolution requirements for line pointing are on the order of $0.2 \mu \mathrm{rad}$.

2. Angular resolution requirements for centering are on order of $10 \mu \mathrm{rad}$ for 90 tines larger than the pointing resolutionj.

3. Total gimbal travel required is on the order of $10 \mathrm{mrad}:=10^{5}$ times the pointing resolution).

In the coarse pointing or acquisition mode the anpular field of view of the sensor is extunded (by using largor, $7 \mathrm{~mm}$, surrogate target) to encompass the uncertainty volume as specified by Lawrence Livermore Laboratory. Coarse pointing and centering are performed simultareously (and focus also) on the larger surrogate target. The fine pointing is then arcomplished on the smaller, $1 \mathrm{~mm}$, surrogate target with the centering function inactive. The field of view of the sensor with the small surrogate target is degigned to be slightly larger than the coarse step of the gimbaled mirrors.

Focusing on the Surrogate Target

Focalezror, between actual focus and that desired focus at the surrogate larget is measured in the focus sensing portion of the pointing and focus sensor, and is driven to a null by axially translating the focusing lens in the target chamber. The focusing function is performed simultaneously with the pointing function land hence with the centering lunction since if is simul:aneous with coarse pointing). The desireci position for the focal point is juat behind the focal point of the sur rogate target, (dinscrited in detail in Section 3!.

For the pointing portion of the sensor to work accurately it munt be in focus, therefore the focus control must be working ior coarse pointing (acquisition) as well as inc pointing.

When the real target is put in the chamber the focal position is shifted to a predetermined point behind the center of the target. Neither the locus 
or pointing portion of the pointing ano focus sensor can be operational at this tirse feven if the real target were a good reflcctor, :lie return energy woulci be so far out o: foclis that neither part of the sersor sould function). Therefore this defocus operation must be accomplished without the optical feedback. The detocus would be implemented through use of precision linear transducers (accuracy of $=3 \mu \mathrm{m}$ out of $2 \mathrm{~mm}$ ). The transducer requires a relative accur acy of about 0 ! percent but has no need for absolute accuracy sinc. the focus sensor provides the initial reference.

Path Length $\&$ nsing

The path length scnsor ( $\triangle \mathrm{PL}$ sensor in Figure $2-2$ ) is in the alignmerit iaser beam prior to the pinhole assembly. The alignment lase $r$ is utilized in the node lock d operation. A portion of the energy is split off for reference, modulated an: heterodyned with the reflected energy from tha surrogate target. The modulation is such that the scusor eutput is nulied when the pathlength is maiched. : ubsequent chains are nulled so that the chains are matched against a common null so that absulute acciracy is not needed. Exact operation of the seisor is described in bection 7.

The system aspects of $\triangle P L$ sensing are the problem of insuring that the reilected (nergy gets back through the system, especially the pinhole. When Shiva is aligned according to the pointing and focus sensor, very little energy wiil return is the pinhole, since the 'xcus criteria is near the focal point of the si rogate target rather than a ine center. Further if the re are non-syrnmetric aberrations in the system the return beam will not be pointed correctly for maximum return enea $\mathrm{gy}$. (Even though the focused beam is exactly at the desired poirt on the targetl. To get maximum energy through the pinhole, the focus lens is shifted so that the focal point is at the center of the target. A locus search mode may be required to maximize the energy through the pishole as spherical abarrations in the return path will tend to shift the loca: point away 'rom the pinhole even when the foral point is exactly at the cester of the target. The aberrations in the return path should never cause a pointing or focus error such that the return signal cannot be found; i. e. focus and pointing errors would only reduce the $S / N$, It should be nuted that the $\triangle P L$ sensor provides an interesting check on the effect of laser rhain aberration on pointirg and focusing. 


\section{OPERATING PROCEDURE}

This section describes in datail the procedure for alignment of the total system, which includes the use of the master console and portable test boxes. The total system is comprised of a number of subsystems which hav't to be aligned such that together they form an int:gral . 'T. The alignment for each subsystem is treated separately in a logic.. squence. When all the subsystems have been aligned in the sequence described, the total system will be aligned and rcady to perform the physics experiments.

The wenty main laser chains may be aligned individually or several simultaneously, depending on the number of persennel and portable test boxes available. If the main laser platform is stable enough it may be possible to align each chain as it is installed. The optimum method will depend on the system assembly procedure.

The master oscillator, final pointing, centering ard focusing are aligned automatically from the master console. All automatic controls have the capability of being operated manually (remote manull mode) from the naster console if necessary or desired. The alignmeint of the finhole to the beam splitting array is accomplished by the remote manual method, from the master console. All other alignment mechanisms ars manially adjusied with the aid of a portable test box.

\section{Controls and Display Panel Description}

\section{Master Console}

Prime factors influencing the design of the master console were reliability, ease of use and the ability to be easily changed as the systen matures. Careiul component selection, such as solid state instead of incandescent indicators, high reliability switches and minimum complexity were deemed essential for systen reliability. Controls were kept to a minimun, without compromising the usefulness of the console, and a logical system of status indicators and araiog cisplays enable the console to be easily used. The front pasel is horizontally divided into lcsical sections. See Figare 2-14, The wiring for each section is an inlegral part of the panel and is connected to the rest of the console by connectors. Each section conplete with its own 


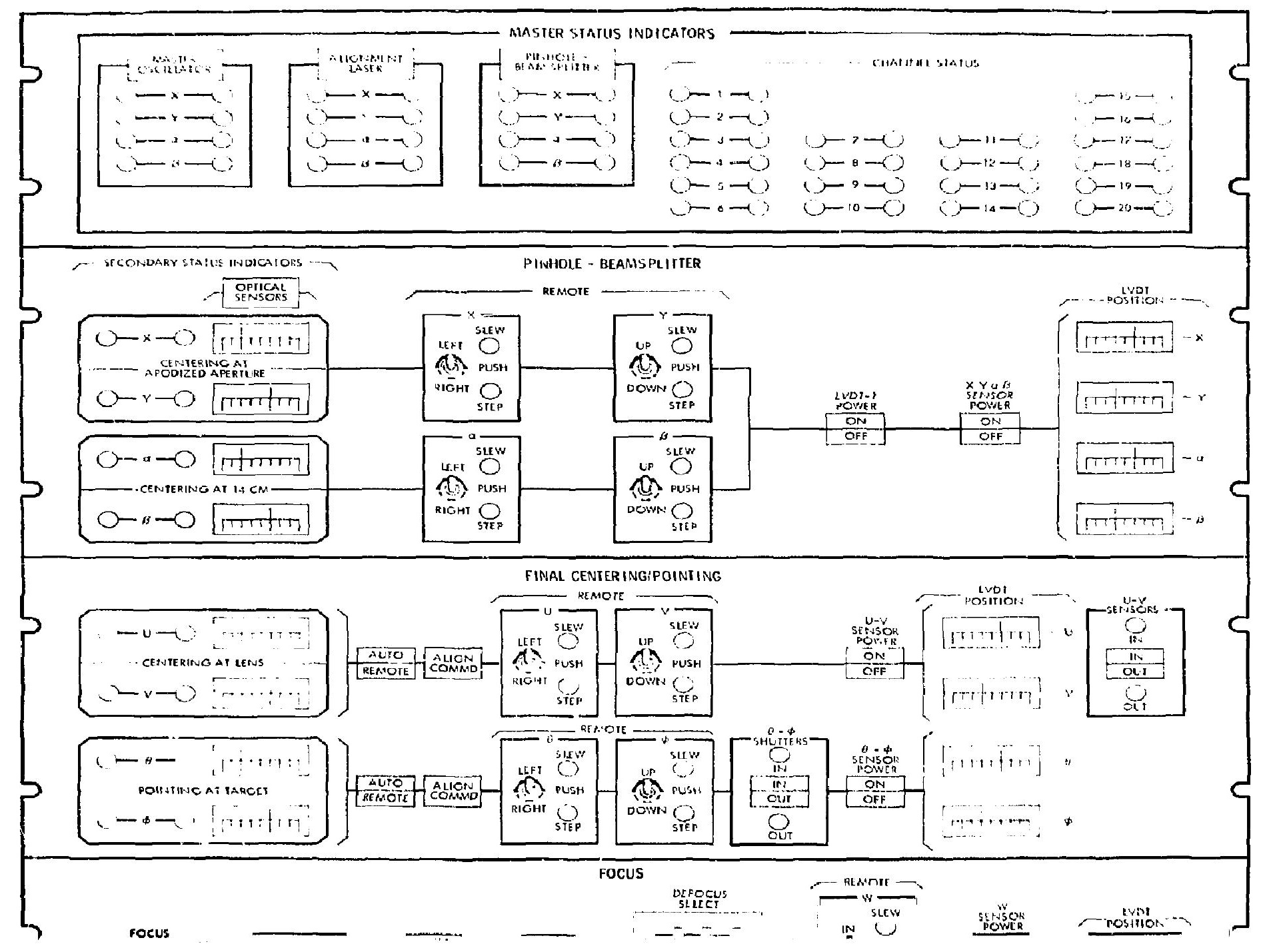




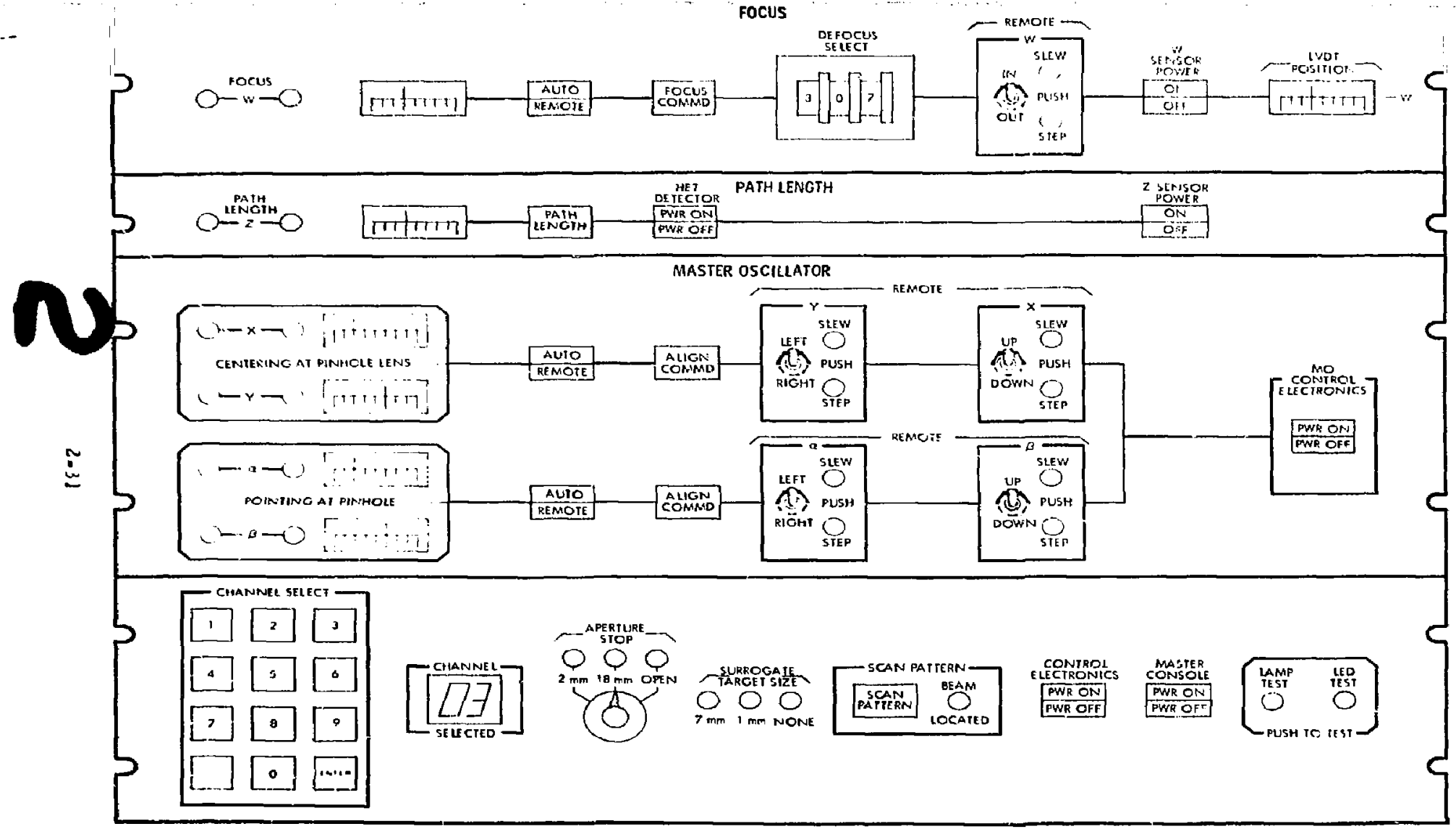

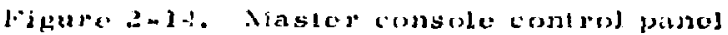


wiring may be easily and completely removed, permittiry the insertien of a new completely wired janel with ease.

\section{Portable Test Box}

The portable test box is a simple, reliable design and is completely self contained requiring no external power supplies or battery power. See Figure 2-15. It consists of a number of electro-mechanicai analog meters that have range sersitivity switches. The meters are driven directly from the buffered sensor outputs, range sensitivity is controlled by switching passive elements. The sensor signal cables are mated to the test box via connectors, allowing the iest box to be moved around easily. The mechanical construction is a rugged design permitting normal laboratory roughhandling. The relatively low cost of the test box makes it attractive to have a number of them availabie so that parallel efforts can be used for alignment.

\section{Alignment Procedure}

Each subsystem procedure begins with a description of which elements are to be aligned. The description is then followed by a step by step alignment procedure format which includes the use of the master console and portable test box.

\section{Alignment Laser to Pinhole Assembly}

The first procedure describes how the alignment laser beam is centered on both the lens and the pinhole. The lens centering is accomplished by

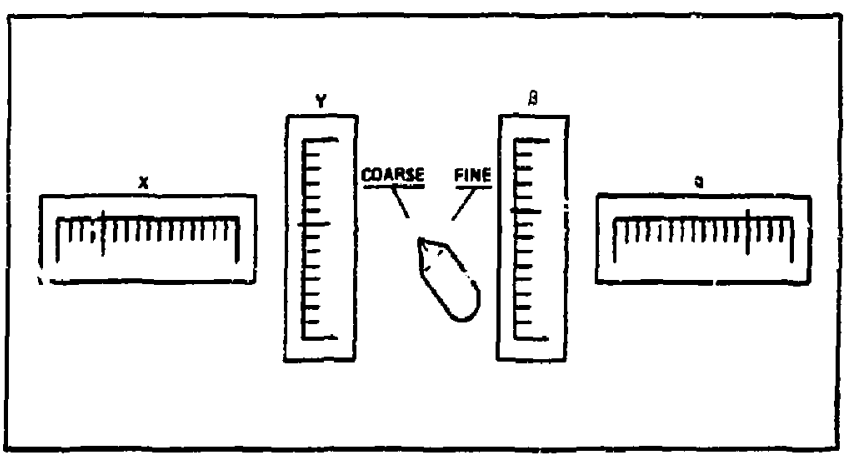

Figure 2-15. Portabin test box 
varying the $X: Y$ axen adjustments and the pinhole centering by varying the $\alpha, \beta$ adjustments. 13:am position is sensed by the $X, Y, \alpha, \beta$ optical sensor that is mounted on tixe same optical bench as the pinhole assembly (see Figure 2-16). Sensor information is transmitted, via a cable, to a portable test box at the $X, Y, \alpha, \beta$, adjustment mechanism. Before the alignment can be attempted the alignment laser and the sensor must be activated via the master console.

Prealignment procedure:

1. Turn on master console:

- select MASTER CONSOLE "PWR ON".

2. Turn on sensor power:

- $\operatorname{select~} X, Y, \alpha, \beta$ SEXSOR POWER "ON"

3. Iurn on alignment laser:

- select ALIGNMENT LASER "ON" and "MOD"

4. Connect the portable test box to the pinhole sensor cable

5. Set COARSE/FINE switch to COAR.SE, on the portable test box.

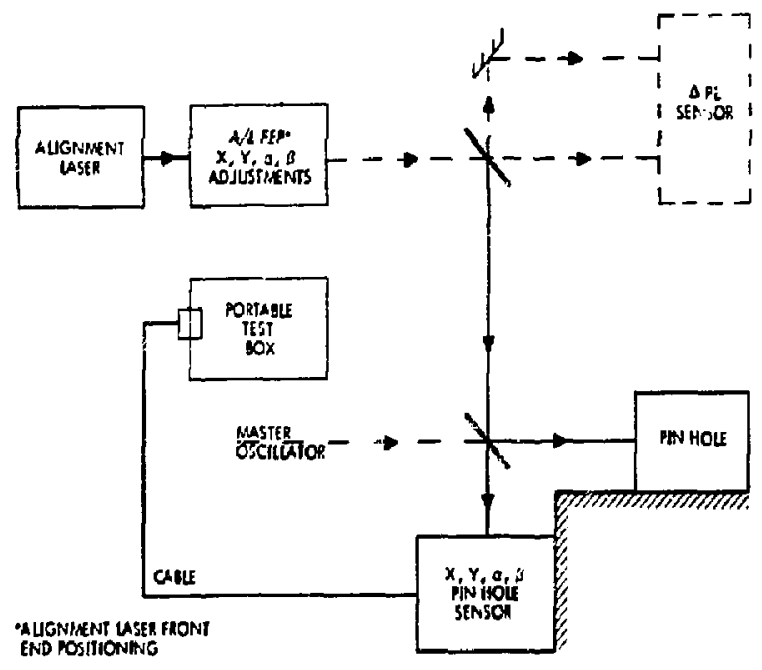

Figure 2-16. Alignment laser to pin hold alignment 


\section{Alignment procedures:}

6. Alternately null lens centering and the pinhole centering with the $X, Y$, and $\alpha, \beta$ controls respectively. The $c$. ter zero analog meters, on the portable test box (PTB), indicaie the intaraction of the adjustments.

7. After obtaining coarse nulling in the $X, Y, \alpha, \beta$ axes change to FINE setting on the PTB and readjust for fine nulls.

Alignment is completed when all four axes are nulled within the prescribed limits (marked on meters). At this point the ALIGNMENT LASER MASTER STATUS INDICATORS, on the master console, should have changed from red to green.

When the optical sensor power is turned off a protective shutter is indexed in front of the sensor lens for protection from the master oscillator beam. When the sensor power is turned on the shutter is indexed out automatically.

\section{Apodized Aperture and Laser Chain Centering}

The beam exiting the pinhole passes through an indexed aperture stop, then through an $X, Y, \alpha, \beta$ positioning mechanism to the beam splitting array. The $X, Y, \alpha, \beta$ positioning mechanism is remotely adjustable from the master console, actual position is sensed by LVDT's. The inaster console display: the LVDT position for each axis.

Each beam leaving the beam splitting array may be positioned by a manually adjusted $X, Y, \alpha, \beta$ (and $Z$ ) mechanism. By means of this latter mechanism the beam may be centered on the apodized aperture, by the $X, Y$ axes adjustments, and centered on the output lens of its main laser chain, by the $\alpha, \beta$ adjustments. Both mechanisms utilize the same set of sensors an $\mathrm{X}, \mathrm{Y}$ sensor and an $\alpha, \beta$ sensor (see Figure 2-17).

The initial gystam alignment should be attempted by selecting a laser chain that has a relatively short distance between the front end positioning (FEP) and the apodized aperture. This selection will minimize the effect of the $a, F$ adjustment on the $X, Y$ error. The remote manually adjusted 


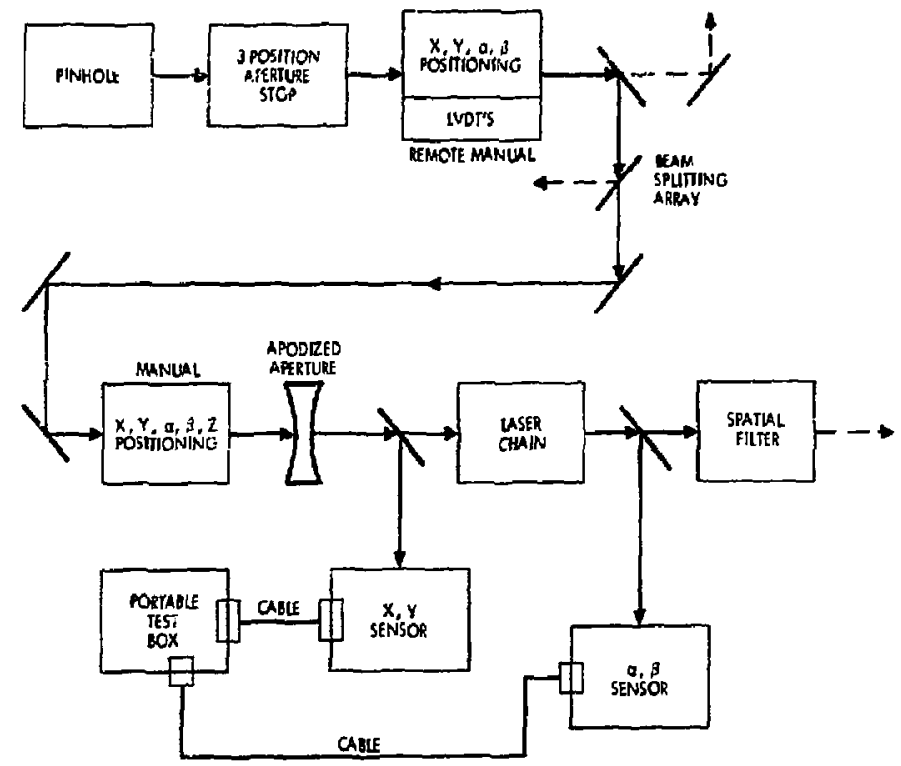

Figure 2-17, Apodized aperture and laser chain alignment

mechanism should first be positioned in the approximate null LVDT position for all axes. The manually adjusted mechanism can th:n be used for beam centering on the apodized aperture and the laser chain oitput lens. Itie alignment procedure is then as follows:

P:ealignment procedure:

1. Turn on master console:

- Belect MASTER CONSOLE "PWR ON"

2. Turn on sensor power:

- select $\mathrm{X}, \mathrm{Y}, \alpha, \beta$ SENSOR POWER "ON"

3. Turn on LVDT sensor power:

- select LVDT-1 SENSOR POWER "ON"

4. Turn on alignment laser:

- select ALIGNMENT LASER "ON" and "MOD" 
5. Connect the portable test box.

6. Select laser chain being aligned:

- depress chain number on CHANNEL SELECT keyboard and then "ENTER" key.

7. Turn on control electronics:

- select CONTROL ELECTRONICS "PWR ON".

8. Select aperture stop:

- turn APERTURE STOP switch to "18 mm".

Alignment procedure:

9. Using the REMOTE PINHOLE-BEAM SPLITTER controis, on the master console, adjust the $X, Y, \alpha, \beta$ mechanism until the LVDT position indicators are nulled.

10. Observe the analog displays on the portable test box and manually adjust the $X, Y$ axes until coarse : ull is obtained (COARSE/FINE switch in the COARSE position). Manually adjust the $\alpha, \beta$ axes until coarse null is obtained. An iteration process will be involved because of the interaction of the $X, Y$ axes and the $\alpha, \beta$ axes adjustments. The remote manual adjustments have electronic cross-coupling compensation to significantly reduce or eliminate this problem.

11. When coarse nulling has been achieved, switch the COARSE/FINE switch on the portable test box ts FINE and repeat step 10 until best nulling is obtained.

Other laser chains may now be aligned with the aid of the portable test box. For laser chaing where the distance from the FEP to the apodized aperture is relatively large the $\alpha, \beta$ adjustnent introduces a significant $X, Y$ error. As each chain is aligned the SECONDAR $Y$ STATUS INDICATORS, on the master console, will change from red to green (each chain has to be "selected" via the CHANNEL SELECT keyboard). 
After all chains have been aligned, cycle through the channels with the CHANNEL SELECTOR and note the + or - errors on the $X, Y$ and $\alpha, \beta$ optical sensor analog displays on the master console. If deviations a re all within tolerance adjust the FEP, using the remote manual controls, to split the difference between all chains, If certain chains are not within specification readjust the offending chain(s) with the portable test box.

\section{Pointing and Focus Sensor Alignment to Reference}

Prior to attempting the final pointing $(\theta, \phi)$ and focusing $(W)$ the pointing/ focus sensor has to be aligned to the incoming beam, for each chain. The sensor unit has its own adjustment mechanism and is aligned with the aid of a portable test box. The reflected beam from the target must be prevented from reaching the sensor during sensor alignment. A shutter that is indexed in interrupts the beam, preventing any piosibility of a reflection (see Figure 2-18).

A similar shutter arrangement is provided for the retro-reflector. During sensor alignment the :etro shutter is open, for system alignment it is closed (see Figure 2-17). The sensor alignment is as follows:

Prealignment procedure:

1. Turn on master console:

- select MASTER CONSOLE "PWR ON"

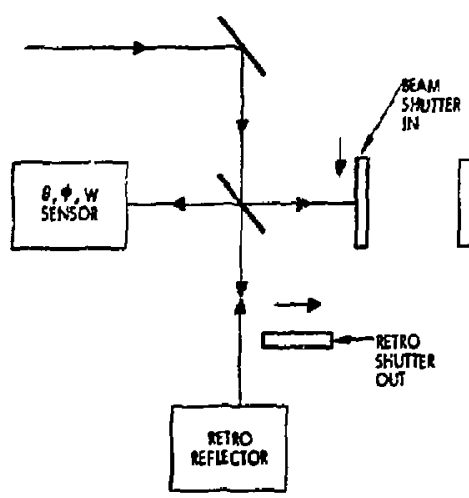

a) SENSOR ALIGIMENT

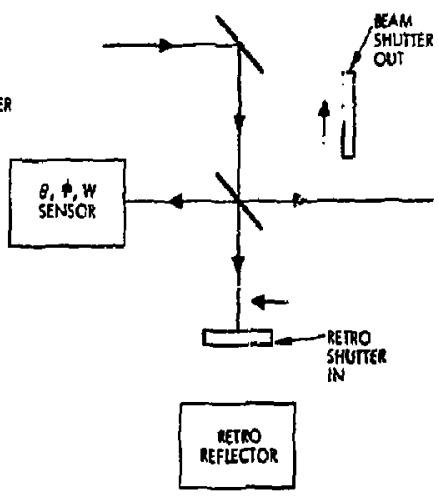

b) SYSTEM ALIGNENT

Figure 2-18, Alignment for sensor and oystem 
2. Turn on sensor power:

- select $\theta-\phi$ SENSOR POWER "ON"

3. Turn on alignment laser:

- select ALIGNMENT LASER "ON" and "MOD"

4. Select aperture stop;

- Turn APERTURE STOP switch to " 2 mim"

5. Select laser chain:

- Depress chain rumber or CHANNEL SELECT keyboard then "ENTER" key.

6. Close bean shutter:

- Select $\theta, \phi$ SHUTTERS 'IN"

(Note: retro shutters are also opened by closing beam shutters)

7. Connect portable test box to sensor.

Alignment procedure:

8. Adjust sensor centering mechanism until nulls are obtained on analog displays on test box.

9. Turn APERTURE STOP switch to " $18 \mathrm{~mm}$ ".

10. Adjust sensor angle mechanism until nulls are obtained on analog displays.

11. Turn APERTURE STOP switch to "2 mm".

12. Affirm that angle null does not change. If null does change re-adjust and check nulls with " $2 \mathrm{~mm}$ " and " $18 \mathrm{~mm}$ " aperture stops.

Other laser chain $\theta, \phi$ and $W$ sensors may be aligned in a similar manner.

\section{Automatic Point/Center/Focus on $7 \mathrm{~mm}$ Surrogate Target}

The final pointing, centering and focusing may be done either automatically or in the remote manual mode, both from the master console. The 
final alignment is done in two stages, coarse and fine. The itr st stage (coarse) is accomplished using a $7 \mathrm{~mm}$ diameter sur rogate target, the second stage (fine) is accomplished using a $1 \mathrm{~mm}$ diameter surrogale targul. The centering mechanisti: is a single resolution derice, consisting of only the coarse stepping motor. The pointing mechanism is a dual resolution device, consisting of coarse and fine stepping motors.

During the coarse alignment ( $7 \mathrm{~mm}$ diameter target) the ine pointing serro is caged such that it remains in the center null position of the coarse pointing servo steps. The coarse pointing servo is controlled with the centering servo such that when the centering servo is moved the pointing servo is moved the same amount. The fine pointing servo, however, can move independently from the centering servo, when only fine pointing is being commanded (See Figure 2-19).

The master console provides both optical sensor and LVDT position information. The LVDT sensors indicate actual positios information, which is necessary for situations where the optical sensor has not yet acquired the beam. If the optical sensor is unable to lacate the beam, then an

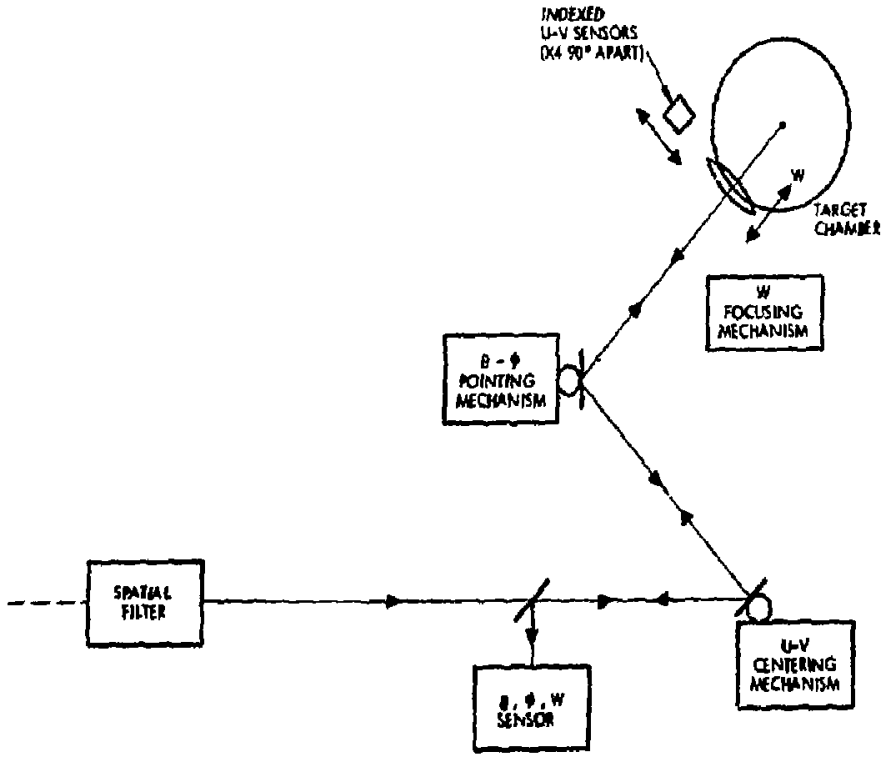

Figure 2-19. Final pointing/centering/focusing 
internally nicropressor guacrated scan pattern is initiated. At the beam is within the boundaries defined by the pointing and centering mechanisms limits then the scan pattern will locate it.

The optical $\mathrm{U}-\mathrm{l}$ cuntering sensors are automatically indexed in, at the larget chamber lens, when pover is applier to them. Indicators are provided on the master console to display the position of the $U-V$ sensors. When power is removed from the U-V sensors then they at e automatically indexed out (Sec Figure 2-1y). The automatic alignment procedure is as follows:

Prealignment procedure:

1. Turn on master console:

- select MaSter console ' PWR oN"

2. Turn on sensor power:

- select o-d SENSOR POWER 'ON'

- select U-Y SENSOR POWER 'OX

- select W(focus) SENSOR POWER 'ON'

3. Turn on alignment laser:

- select ALIGNMEXT LASER "ON" and "MOD"

4. Select "18 mm" on APERTURE STOP switch.

5. Open sensor shutters:

- select $\theta-\phi$ SHUTIERS "OIJT"

6. Observe TARGET SIZE indicators:

- $\quad 7 \mathrm{~mm}$ " LED should be on (red)

7. Pul centering, pointing and focusing in automatic mode:

- select "AUTO" on $\theta-\phi$ sensors

- select "AUTO" on U-V sensors

- seli et "AUTO" on W sensor 
8. Set up alignment commani:

- select "ALIGN COMMAND" on $\theta-\phi$ sensors

- select "ALIGX̃ COMMAXD" on U-V sensors

- select "FOCUS COMMAXD un W sensor

Alignment procedure:

9. Turn on control electronics:

- select CONTROI ELECTRONiCS 'PWR ON"

If all channels a!igned correctly the MASTER LASER CHAIN ST ATUS indicators will all be green. Any channel that did not align will be revealed by a red indicator. Fote any ofiending channels and proceed as lollows:

10. Turn off control electronics:

- sclecr control E.LCTRONiCS "PWR OFF"

11. De-select the alignment command:

- depress "ALIGN COMMAND" on $\theta-\phi$ sensors | switch should

- depress "ALIGN COMMAND" on U-V sensors $\begin{aligned} & \text { change from } \\ & \text { red to green }\end{aligned}$

- depress "FOCUS COMMAND" on W sensor illumination.

12. Isolate offending channel:

- depress channel number on CHANNEL SELECT keyboard and depress "ENT ER" key.

Assuming that the re are no component failures the pointing cr focus optical sensors have probably not acquired the beam.

13. Initiate scan pattern:

- selert "SCAN PATTN"

Wien optical sensors have acquir ed the beam the scan pattern is terminated. Repeat the above procedure for all offending channels then repeat steps 8 and 9 to complete the alignment, When final pointing, centering and focusing (coarse) alignment is completed:

14. Turn off control electronics

- selecl "CONiROl electronics "PWR ofF" 
For fine alignment from this point the procedure is the same except. that the surrogate target size is now $1 \mathrm{~mm}$, as follows:

15. Replace $7 \mathrm{~mm}$ diameter surrogate target with the $1 \mathrm{~mm}$ diameter surrogate target.

16. Observe TARGET SIZE indicators:

- $1 \mathrm{~mm} "$ LED should be on (red)

17. Turn on control electronics:

- sểet CONTROL ELECIRONICS "PWR ON"

If ali channeis aligned themselves correctly the MASTER LASER CHAIN STATUS indicators will all be green. Any channel that did not align will be revealed by a red indicator. Note any offending channels and proceed as follows:

13. Turn off control electronics:

- select CONTROL ELECTRONICS "PWR OFF"

19. De-select the alignment command:

- depress "ALIGN COMMAND" on $\theta-\phi$ sensors | switch shoula

- depress "ALIGN COMMAND" on U-V sensors $\begin{aligned} & \text { change from } \\ & \text { red to green }\end{aligned}$

- depress "FOCUS COMMAND" on W sensor fillumination.

20. Isolate offending channel:

- depress channel number on CHANNEL SELECT keyboa::d and depress "ENTER" key.

21. Select remote control:

- select REMOTE on $\theta-\phi$ sensors.

- select REMOTE on U-V sensor.

- select REMOTE on W sensor.

Final pointing, centering and focusing is now in remote manual mode. Assuming that coarse alignment was achieved (with $7 \mathrm{~mm}$ diameter surrogite target) then only the "single step" mode should be used for fine alignment. 
By observing the LVDT analog displays and the optical sensor analog displays it should be possible to adjust the mechanisms within the specified limits.

\section{Remote Manual Pointing/Certering/Focusing onto Target Chamber}

The remote manual mode of final adjustments is provided primarily for a back-up system in the event the automatic mode is non-functional. However, the remote manual mode is also useful for checking out the final mechanisms. Two modes of operation are possib!e wit's the system operated in remote manual, they are:

(i) Slew mode

(ii) Stepping mode

If the "SLEW" button is depressed a continuous stream of pulses is appilied to the stepping motors. If the "STEP" button is depressed the stepping motors are only moved one step at a time. The procedure for placing the system in the REMOTE MANUAL mode (for $\mathrm{fi}$ : 1 pointing, centering and focusing) is as follows:

1. Turn on master console:

- select MASTER CONSOLE 'PWR ON'

2. Iurn on sensor power:

- $\operatorname{select} \theta-\phi$ SENSOR POWER "ON"

- select U-V SENSOR power "ON"

- select W SENSOR POWER "ON"

3. Turn on alignment laser:

- select ALIGNMENT LASER 'ON" and "MOD"

4. Select aperture stop:

- Turn APERTURE SIOP switch to "18 mm"

5. Open sensor stutters:

- $\operatorname{select} \theta-\phi$ SHUTTERS "OUT" 
6. Select remote manual mode:

- select "REMOTE" on $\theta$ - $\phi$ sensors

- select "REMOTE" on U-V sensors

- selecl "REMOTE" on W sensor

7. Turn or control electronics:

- select CONTROL ELECTKONICS 'PWR ON"

The final pointing, centering and focusing mechanisms may now be operated by the remote manual controls. For the $\theta-\phi$ and $U-V$ mechinisms LEFT/ RIGHT SLEW/STEP or UP/DOWN SLEW/STEP may be selected. For the W (focus) mechanism IN/OUT SLEW/STEP may be selected. The W nechanism can also be moved in or out by means of the thumbwheel switch and the 'DEFOCUS COMMAND' as follows.

8. Dial appropriate number on thumbwheel switches

9. Select "IN" or "OUT" with toggle switch

10. Depress "DEFOCUS COMMAND"

Focus mechanism will move by the amount commanded in the direction indicated by the toggle switch.

Path Length Matching $-\triangle P L$

The path length measuring technique relies on receiving a beam raturn that is reflected from the target. The system, therefore, has to be fully aligned before any path lergth measurements can be made. A simplified schematic of the path length measurement system is shown in Figure 2-20.

To obtain the reflected beam back through the optical systen it is necessary to focus at the center of the target. Some fine adjustment may be necessary on the final pointing and focusing in order to pass the beam back through the pinhole. Only one channel at a time may be adjusted, all other beam returns must be prohibited from reaching the pinhole. Blocking the return beams on channels not being adjusted is done by the existing pockle cells.

The path length is varied by manaully adjusting the mechanism located between the beam splitting array and the apodized aperture. The path length 


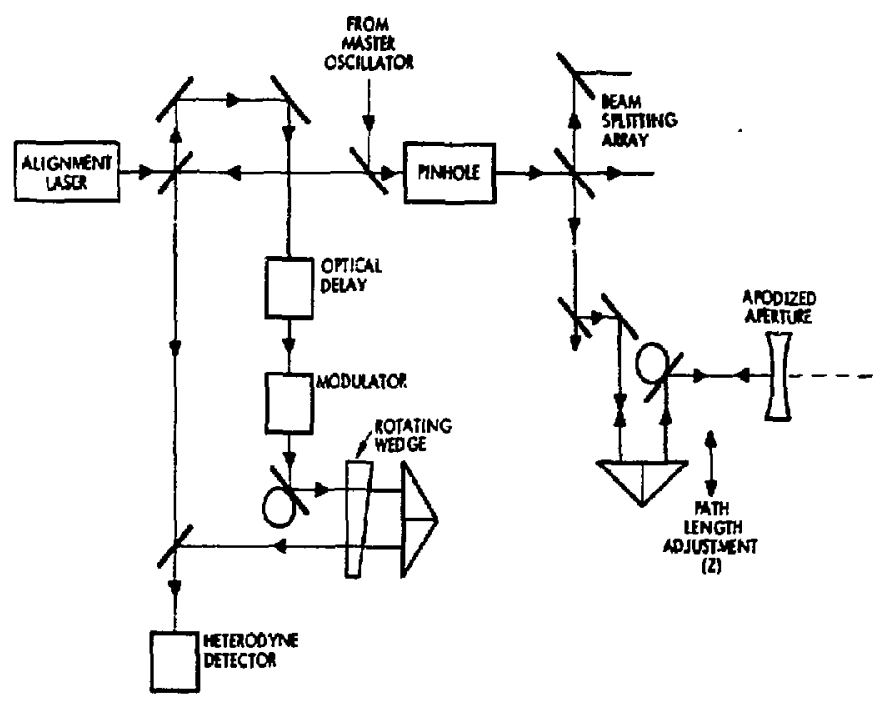

Figure 2-20. Path length measurement

mechanism is then adjusted until a null display is obtained on the portable test box. The procedure is as follows:

Prealignment procedure:

1. Turn on master console:

- select MASTER CONSOLE "PWR ON"

2. Turn on heterodyne detector:

- select HET, DET. "ON":

3. Turn on alignment laser:

- select ALIGNMENT LASER "ON" and "MOD"

4. Select aperture stop:

- turn APERTURE STOP switch to "OPEN" 
5. Put focus in remote manual mode:

- select "REMOTE" on focus panel

6. Turn on focus sensor:

- select W SENSOR POWER "ON"

7. Connect portable test box.

Alignment procedure:

8. Select channel to be adjusted

- Depress channel number on CHANNEL SELECT key'joard and depress "EXTER" key

9. Allow pockle cell to be "opened":

- Depress "PATH LENGTH" switch

10. Focus beam at center of target:

- Use "IN": or "OUT" on toggle switch and "STEP" mode until beam is reflected through pinhole.

11. Adjust path length:

- Manually vary $Z$ mechanism until null is obtained on portable test box analog display.

Remaining channels can then be adjusted by repeating steps 8 through 11 . Master Oscillator Alignment

The master oscillator alignment is accomplished automatically from the master console. Provision is made, as with all other automatic functions, for a remote manual mode of operation. It is only $: x$ c:sssary to align the master oscillator to the pinhole, since the pinhoie is aigned to the rest of the system using the alignment laser (See Figure 2-21). The alignment procedure is as follows:

Prealignment procedure:

1. Turn on master console:

- select MASTER CONSOLE "PWR ON" 


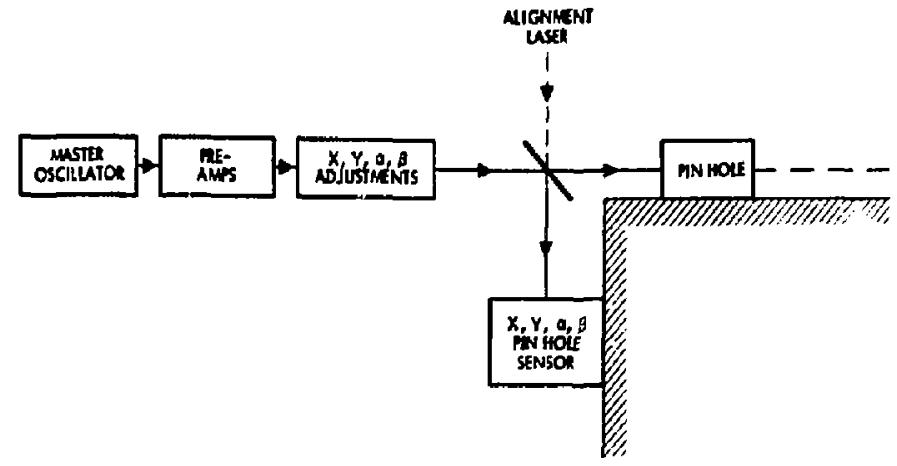

Figure 2-21. Master oscillator to pinhole alignment

2. Turn on sensor power:

- select $X, Y, \alpha, \beta$ SENSOR POWER "ON"

3. Select automatic mode:

- select "AUTO" on master oscillator panel.

4. Turn on master oscillator:

- request signal is sent to Shiva when CONTROL ELECTRONICS (STEP 5) is activated. Shiva controls on/off of the master oscillator.

Alignment procedure:

5. Turn on control electronics:

- select MO CONTROL ELECTRONICS "PWR ON"

\section{Depress "ALIGN COMMD".}

The master oscillitor should no!s be aligned to the pinhole, verification is provided by the MASTER STATUS INDICATORS for the MASTER OSCILLATOR LED illumination which should be green. For operation in the remote manual mode step 3 would read:

3. Select remote manual mode:

- select "REMOTE" on master oscillator panel. 
The alignment procedure would then be:

Alignment procedure:

5. Turn on control electronics:

* select Mo control electronics "PWR oN"

The master oscillator may now be aligned using the remote manual controls. The operator can observe the error on the master console analog display of the $X, Y, \alpha, \beta$ sensors.

\section{INTERCONNECTION AND INTERF ACINC}

The electro-mechanical alignment system requires sensor information at the master console from various locations. The master console sends control commands to the alignment mechanisms and exchanges status and other information with the Shiva system. The system design approach used was to attempt to minimize the number of interconnections, thereby increasing the reliability while keeping the complexity and cable costs to a minimum.

Iable 2-18 summarizes many of the system interconnections between the various sensors, adjustment mechanisms and the master console. Each unit, such as a sensor, is listed with a brief description of the signal, the source and destination of the cable. In addition to the cables listed there are others which are used for monitoring the various shutter positions and are fed into the CAMAC interface system. The shutter position information is used by the Shiva system for making a fire decision. Figure 2-22 gives a generalized schematic of the alignment system and indicates the various inter connections.

There are three basic types of interface between the electromecisnical alignment system and the Shiva system, they are; discrete, computer format and CAMAC. The discrete interface has been reserved for vital information or command functions because they are a direct connection exhibiting low complexity and high reliability. The number of discrete interfaces have been kept to a minimum because of the information capacity inefficiency.

The computer format interface is used for information exchange that is not vital to the eystem operation, so that a failure in this interface will not 
Table 2-18. System interconnection summary

\begin{tabular}{|c|c|c|c|}
\hline Sensor or Mechanism & Function & Source & Destination \\
\hline $\begin{array}{l}\text { Master oscillator } X, Y, a, \beta \\
\text { mechanism }\end{array}$ & Motor dritues & Master console & $M O-X, Y, O, \beta$ nechanism \\
\hline $\begin{array}{l}\text { Alignment laser } X, Y, \alpha, \beta \\
\text { mechanism }\end{array}$ & \multicolumn{3}{|c|}{ NONE - MANUAL ADJUSTMENT } \\
\hline $\begin{array}{l}\text { Pinhole } X, Y \text { or } \alpha, \beta \\
\text { sensor }\end{array}$ & $\begin{array}{l}\text { 1. Sensor information } \\
\text { 2. Sensor information } \\
\text { 3. Power }\end{array}$ & $\begin{array}{l}\text { Sensor } \\
\text { Sensor } \\
\text { Master console }\end{array}$ & $\begin{array}{l}\text { Master corsole } \\
A L / X, Y, a, B \text { mechanism } \\
\text { Sensor }\end{array}$ \\
\hline 3 position aperture stop & $\begin{array}{l}\text { 1. Drive signal } \\
\text { 2. Position }\end{array}$ & $\begin{array}{l}\text { Master console } \\
\text { Aperture stop }\end{array}$ & $\begin{array}{l}\text { Aperture stop mechanism } \\
\text { Master console }\end{array}$ \\
\hline $\begin{array}{l}\text { Pinhole-bean splitter } \\
X, Y, a, \beta \text { medium }\end{array}$ & $\begin{array}{l}\text { 1. Motor arives } \\
\text { 2. LVDT signals } \\
\text { 3. LVDT power }\end{array}$ & $\begin{array}{l}\text { Master console } \\
\text { P-BS mechanism } \\
\text { Master console }\end{array}$ & $\begin{array}{l}\text { P.BS mechanism } \\
\text { Ma ster console } \\
\text { P-BS LVDT's }\end{array}$ \\
\hline $\begin{array}{l}\text { Beam splitter to apodized } \\
\text { aperture mechanism }\end{array}$ & \multicolumn{3}{|c|}{ NONE- - MANUAL ADJUSTMENT } \\
\hline $\begin{array}{l}\text { AA-main laser chain } \\
X / X \text { sensor }\end{array}$ & $\begin{array}{l}\text { 1. Sensor signals } \\
\text { 2. Sensor signals } \\
\text { 3. Power }\end{array}$ & $\begin{array}{l}X-Y \text { sersor } \\
X-Y \text { sensor } \\
\text { Master console }\end{array}$ & $\begin{array}{l}\text { PS-AA mechanism } \\
\text { Master console } \\
X / Y \text { sensor }\end{array}$ \\
\hline $14 \mathrm{~cm} 0, \beta$ sensor & $\begin{array}{l}\text { 1. Sensor signals } \\
\text { 2. Sensor signals } \\
\text { 3. Power }\end{array}$ & $\begin{array}{l}\alpha, \beta \text { sensor } \\
a, \beta \text { sensor } \\
\text { Master console }\end{array}$ & $\begin{array}{l}\text { BS-AA mecharism } \\
\text { Ma ster console } \\
a, \beta \text { sensor }\end{array}$ \\
\hline$\theta, \phi$, W sensor & $\begin{array}{l}\text { 1. Sensor signals } \\
\text { 2. Power }\end{array}$ & $\begin{array}{l}\theta, \phi, W \text { sensor } \\
\text { Master console }\end{array}$ & $\begin{array}{l}\text { Master console } \\
\theta, \phi, W \text { sensor }\end{array}$ \\
\hline $\mathrm{U}, \mathrm{V}$ centering mechanism & $\begin{array}{l}\text { 1. Motor drives } \\
\text { 2. LVDT power } \\
\text { 3. LVDT signals }\end{array}$ & $\begin{array}{l}\text { Master console } \\
\text { Master console } \\
\text { U, V - LVDT's }\end{array}$ & $\begin{array}{l}\mathrm{U}, \mathrm{V} \text { mechanism } \\
\mathrm{U}, \mathrm{V} \text { - LVDTS } \\
\text { Master console }\end{array}$ \\
\hline$\theta . \phi$ pointing mechanism & $\begin{array}{l}\text { 1. Motor drives } \\
\text { 2. LVDT power } \\
\text { 3. LVDT signals }\end{array}$ & $\begin{array}{l}\text { Master console } \\
\text { Ma ster console } \\
\text {, } \phi=\text { LVDT's }\end{array}$ & $\begin{array}{l}\theta, \phi \text { mechanism } \\
\theta, \phi \text { - LVDT's } \\
\text { Ma ster console }\end{array}$ \\
\hline$U, V$ sensors & $\begin{array}{l}\text { 1. Power } \\
\text { 2. Sensor signals } \\
\text { 3. Position information }\end{array}$ & $\begin{array}{l}\text { Master console } \\
U, V \text { sensor } \\
\text { U, V sensor mechanism }\end{array}$ & $\begin{array}{l}\text { U, V senso: } \\
\text { Master console } \\
\text { Master console }\end{array}$ \\
\hline W focusing mechanism & $\begin{array}{l}\text { 1. Motor drives } \\
\text { 2. LVDT power } \\
\text { 3. LVDT signals }\end{array}$ & $\begin{array}{l}\text { Master console } \\
\text { Master console } \\
\text { W - LVDT's }\end{array}$ & $\begin{array}{l}\text { W mechanism } \\
\text { W - LVDT's } \\
\text { Master console }\end{array}$ \\
\hline
\end{tabular}




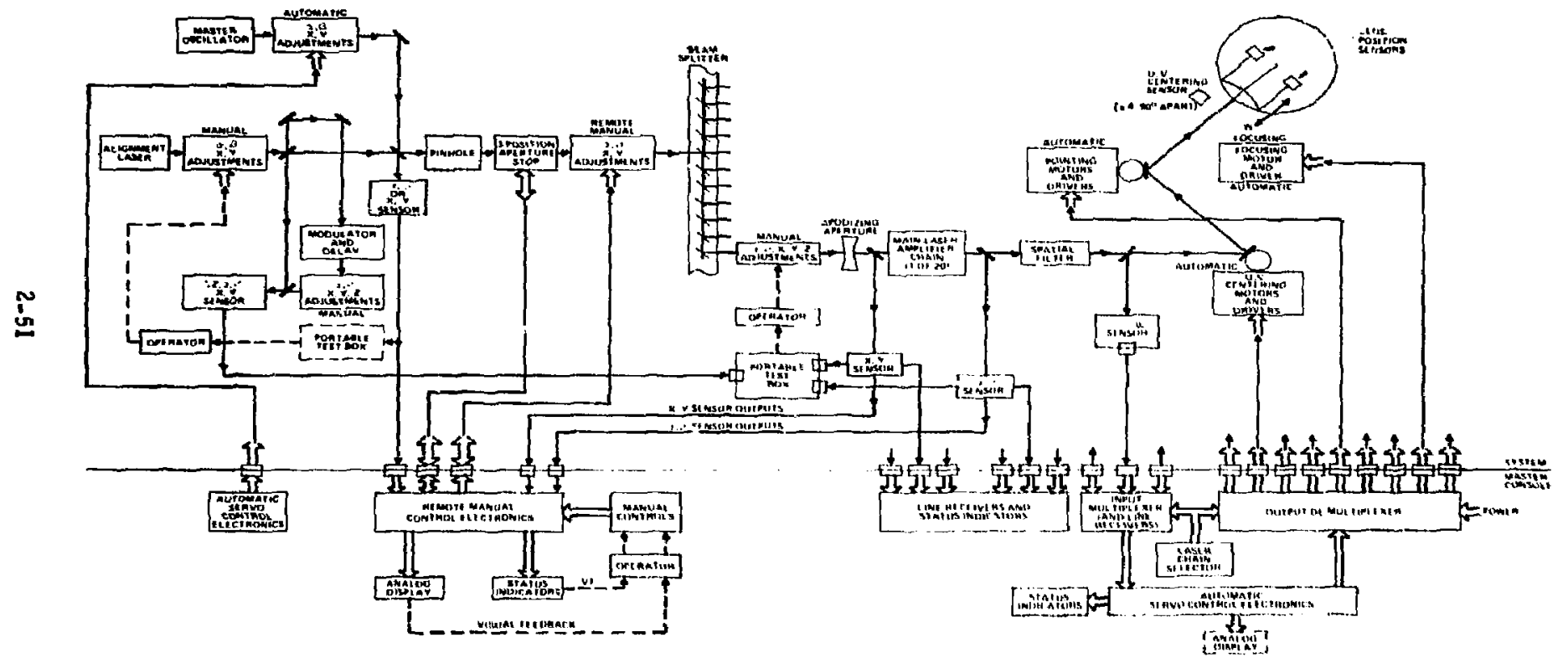

Figure 2-22, System functional diagram 
shut down the system. The master console, for the alignment system, contains a microprocessor which exchanges non-vital information, such as alignment magnitude errors, with the Shiva computer which can either store the information or produce a hard copy readout.

The CAMAC interface is used, for example, in monitoring the shutter positions, information which the Sbiva system requires to make a fire decision. Use of a priority interface system, such as described, will ensure that the system will achieve the maximum reliability for a given set of components. 


\section{POINTING AND FOCUS SENSING}

\section{OVERALL OPTICAL CONFIGURATION}

The main design goals for pointing and focus sensing and the resulting requirements are summarized in Table $3-1$.

\section{Physical Description}

Ite optical configuration for location of the pointing and focus sensor is shown in Figure 3-1. The sensor is located behind the last plate of the reflective polarizer which zets as a beamsplitter. The polarizer and sensor assembly are mounted together rigidly, with the relro, and are calibrated together prior to installation. There is a $\lambda / 4$ plate between the retro and polarizer to rotate the polarization sc that the output of the retro will be reflected from the back of the polarizer. The beam comes from the last spatial filter with an $f / 630$ divergent cone and is focused by the focusing lens to an $\mathrm{f} / 1.58$ cone at the target. The desired focal point for pointing and focus reference is just behind the focal point of the sur rogate target which is a $1 \mathrm{~mm}$ ball. The location of the focal point with respect to the ball is

Table 3-1. Pointing and focus sensing goals

\begin{tabular}{|c|l|}
\hline Coal & \multicolumn{1}{|c|}{ Requirement } \\
\hline Low Cost & Small (under $25 \mathrm{~cm}$ ) \\
& Simple \\
& Loose fabrication tolerances \\
Useability & Easy to align (loose tolerance) \\
& Easy to check \\
Reliability & Simple \\
& Holds alignment - mechanical \\
& and electrical \\
\hline
\end{tabular}




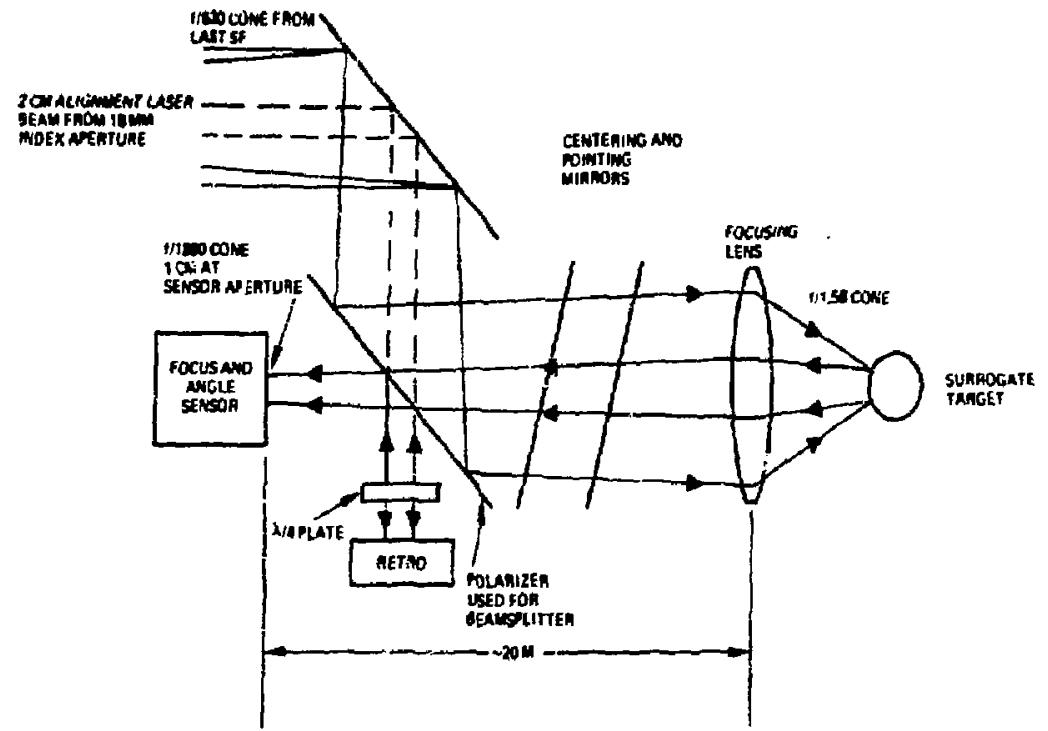

Figure 3-1. Bazeline optical configuration for location of angle and focus sensor

such that after the beam passes back through the lens a second time, the beam converges in an $\mathrm{f} / 1880$ cone. The local point of this cone is $19 \mathrm{~m}$ behind the sensor so that the beam into the sencor is $1 \mathrm{~cm}$ diameter.

The main features of this optical configuration for the pointing and focus sensor are:

1. Full beam is utilized with a small aperture (small sensor).

2. Alignment tolerances are relatively loose.

3. Focus does not occur at center of ball; ther efore, the ball diameter must be known precisely.

The retro which is used for sensor alignment is mounted so that when the small centering reference beam (indexing aperture set to $2 \mathrm{~mm}$ ) hits the beamsplitter the beam going to the sensor from the retro is identically aligned to a beam coming from the target returning exactly on the ontgoing 
beam path. This is shows more clearly in Figure 3-2. The centering reference bean irom the alignment laser is a hout $2.2 \mathrm{cn}$ at the senser. (11 expands from: $2 \mathrm{~cm}$ at the last spatial filter to $2.5 \mathrm{~cm}$ at the locusing lens.) When the $1 \mathrm{~mm}$ ball is used as the surrogate target, the scnsitwity is such that the 80 nm sensor aperture provitis a $\pm 26 \mu \mathrm{m}$ ficlel as measureri at the target. To accommodate the $\pm 1+0 \mathrm{Fm}( \pm 100 \mu \mathrm{m} / \mathrm{pe}$ axis) ficld that is specified, a larger diameter ball is utilized for acquisition." The combinztion of the $80 \mathrm{~mm}$ sensor aperture and a $7 \mathrm{~mm}$ surregate target provides the required acquisition field of $\pm 100 \mu \mathrm{m}$ per axis.

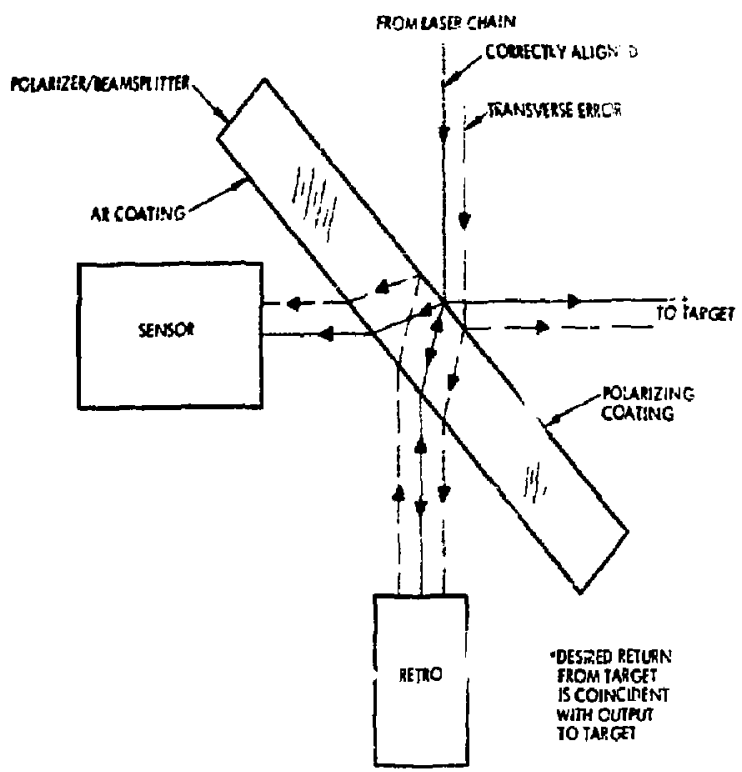

Figure 3-2. Relation of pointing and focus sensor beàmsplitter ani, retro

\footnotetext{
*Sensitivity is inversely proportional to ball diameter - see Appendix. \%ts

Slightly larger than coarse step.
} 


\section{SENSOR DESIGN}

\section{Sensor kequirements}

The impostant factw in sclezting a sensing technique and sensor parameters art arerage signat amplitude and nodulation index. ISte Appendix A fo: tiscussion. I Since the wo factors are usually coniticting, (i.e., increasing one will decrease the other art vice versa) a gooe de sign must strike a batance, In addition, the selection of - timum parancters must be modulated by considerations such as cost and ava lability of components, ease ci fatzication, assembly, and ali gnment of the der:ae. Also, one must consider thi affect of teclinique and parameters on non-i-deal or aberrated optical inpuls and their affect on the operation.

\section{Focus Sensing Technique and Parameter Selection}

The concept for sensing loc:s (see Figure $3-3$ ) is to sample the intensity of the focused beam on either side of the focal plan: and to control focus for a balance between the two. The Figure snows two detectors, $A$ and $B$, located on either side of the focal point at a distance, $7_{0^{*}}$. Detector $A$ is $t t+7_{0}$ and $B$ is at $-Z_{0^{\circ}}$. Figure $3-t$ shows how the error signal modulation is generated. I(7) is the on axis intensity as a function of position, along the axis. 7 is measured from the focal plane. If there is a focus error of i $\Delta 7$ then detector $A$ is at $Z_{0}+\Delta Z$ and detectcr $B$ is at $Z_{0}-\Delta \%$. Alternately zampling the detectors as a function of tine would generate a modulated signa! as shown in the Figure 3.4 where the intensity is modulated from $I\left(7_{0}+\Delta Z\right)$ to

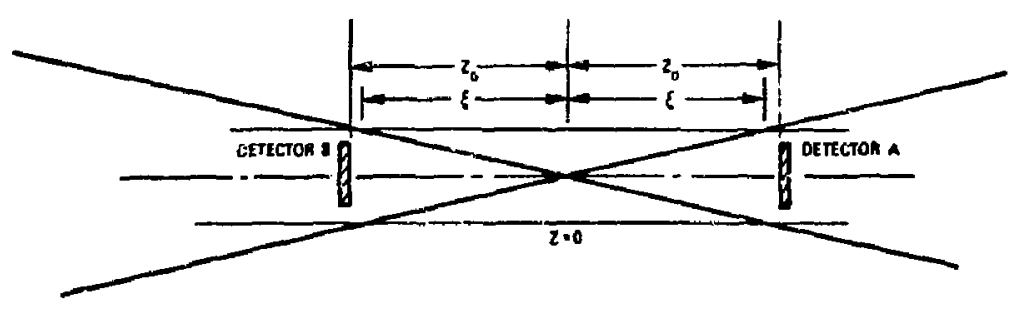

Figure 3-3. Concept for sensing focus 


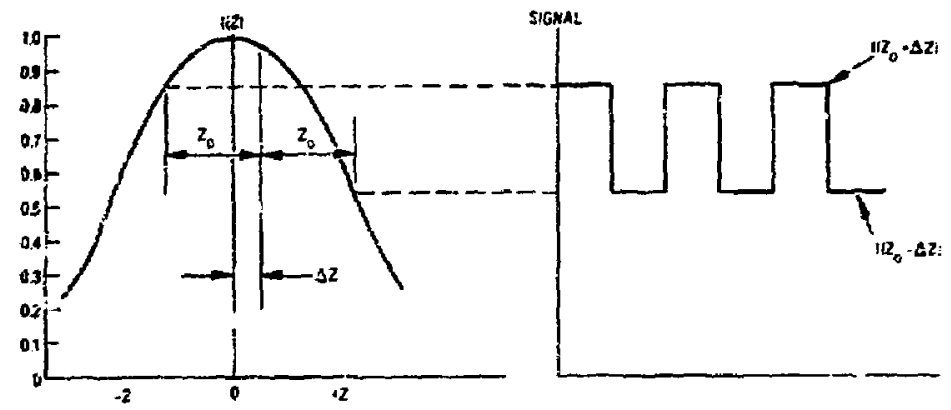

Figure 3-4. Generation of focus error signal

$I\left(Z_{0}-\Delta Z\right)$. If focus is adjusted to mill the modulation, then $\Delta Z$ wouid be zero. The average signal level is $\left.I / Z_{0}\right)$. The modulation index is given by:

$$
M I=\frac{Z_{0} / \xi}{\left[1+\left(z_{0} / \xi\right)^{2}\right]^{2}} \frac{\left(r / \sigma_{0}\right)^{2} \exp -\frac{1}{2} \frac{\left(r / \sigma_{0}\right)^{2}}{1+\left(Z_{0} / \xi\right)^{2}}}{1-\exp 1+\left(Z_{0} / \xi\right)^{2}}
$$

where

$$
\begin{aligned}
& Z_{0}=\text { detector location from focus } \\
& \xi=\text { depth of focus } \\
& t=\text { detector radius }
\end{aligned}
$$

$c_{0}=$ standard deviation of focused blur

It is assumed thet the inpst aperture has been illuminated by a gaussian beam having a $1 / \mathrm{e}^{2}$ diameter equal to the untrance aperture diarneter. Maximum moíulation index (A:I) which is $1 / \xi$ occurs when $r$ equals 0 and 2 equals $\xi$, as one might expect, since this represents sampling the peak intensity at the depth of focus. The averige siģnal amplitude is of course 
proportional to the detector radius, so a zero radius detector is unclesirabic from this point of view. Also a larger radius detector would average a larger portion of the beam, the reby minimizing the effect of optical abcr rations. It is also de sirable to sample further (axially) from the focal plane as the effects of aberration are $;$ educed, because the detector is sensitive to amplitude and not phase. It should be noted that the energy on the real :arget is out of focus, therefore operat ng the sensor at this point tends to make measurements in the location whe :e the enersy is used on the target.

Figure 3-5 shows how MI varies as distector size, $r$, and detector location, $Z_{0}$, are varied. These curves were used to determine (or estimatel the loss in MI for fifferent combinations of detector size and location. It should be noted that these curves, and in tact MI, have been couputed on a theoretical basis, and hence are only valid as estimators of actural performa ice. The estimation however is reasonably accurate bull somewhat pessimistic, i.e., actual performance will be better. Detector size and location were selected by exarnining the data from the optical diffraction analysis and estimating the performance as compared tc an ideal gaussian beam that was 2.5 times the diameter of a diffraction linited beam. The parameters were alvo adjusted to accommodate the physical dimensions of the sensor. For example the detector spacing was uncreased slightly over

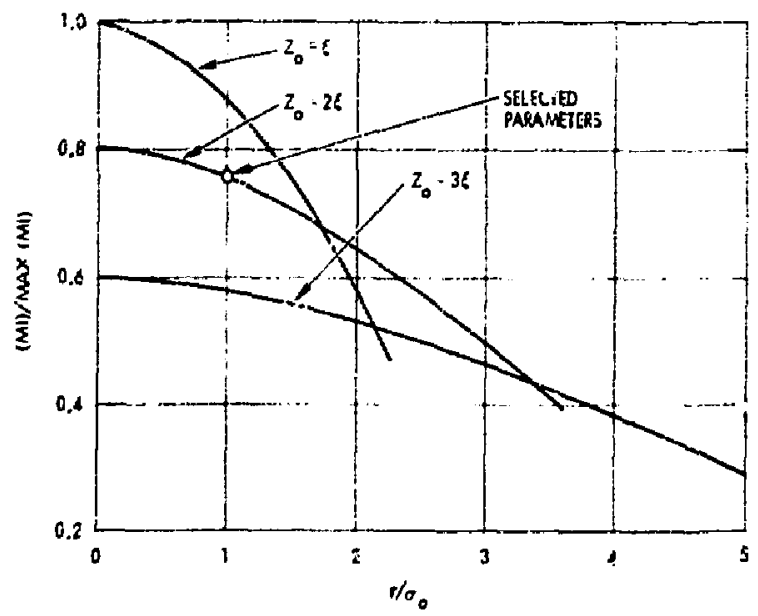

Figure 3-5. Parametric variation of modulation index 
the original space in order to make the sensor easier to fabricate. The increase in spacing was also desirable for the pointing detectors as will be discussed iater. The final spacing for the detector was $Z_{0}: 2 \xi$. The increase in spacing was obtained by giving up a slight (but acceptaile) amount of M: Figure 3-6 shows a thru-focus evaluation of energy on a detector for various detector sizes normalized to dimensions at the target. These curves were made by scanning in the computer) a detector of given racius through the focal plane. The radial location of tha detector was adjusted so that it was located at the same place as a gauad detector would be for null. This is essential since the focus detector and the pointisig delector must utilize the same reference. The curves of Figure 3-6 are for an extreme case, $1 \lambda$ com;, of aberration. The smallest deteclor, $0.8 \mu \mathrm{m}$, is equivalent to sensing the peak intensity since only 12 percent of the energy falls on it at focus. The depth of focus as estimated by this scan is $31 / 2=15.5 \mu \mathrm{m}$. The calculated depth of focus for the 2.5 times diffraction limited case is $13 \mu \mathrm{m}$ so there is fair agreement between the theoretical worst case and a real worst case. From the curve of Figure 3-6, it can be seen that there is little difference in the curve shape (hence little

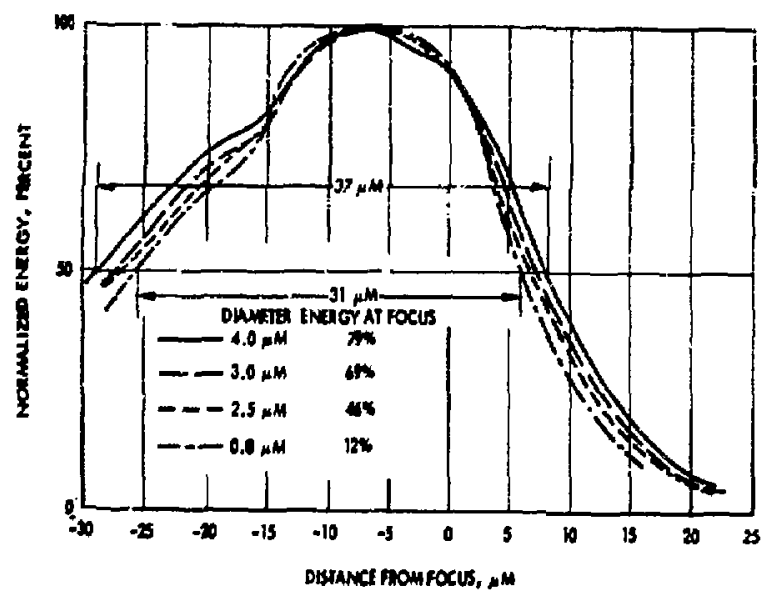

Eigure 3-6. Through-focus evaluation of energy passing through circular aperture centered at null balance of quadrant detector for 1 coma input error 
difference in $M L$ between the $+\mu \mathrm{m}$ detector and the $\mathrm{C} .8 \mu \mathrm{m}$ detector; however, there is a great deal of difference in total ene rgy, 79 percent versus 12 percent. For this reason, the detector size of $4 \mu \mathrm{m}$ is judged to be best, so the detector in the sensor was selected to be equivalent to $4 \mu \mathrm{m}$ at the target. This detector radius is equisalent io $1 \sigma$ fone times the $1 / e$ radius) of the focused blur. From the curve of Figure $3-5$, it can be seen that the selection of paranneters resulied in an $M I 1$ that is io percent of the theoretical maximum $\mathrm{Mi}$. The linal parameters and the basis for their calculation are shown in Table 3-2. The selection of these values was based on evaluation and comparison of many disfraction analys is data from Section 11.

For purposes of $S / N$ calculation an inexpensive detector/amplifier package was tentatively seircted. The detector, a UDI 400, has an NEP of $10^{-10}(\mathrm{~W}, 1 \mathrm{~Hz}, 1.06 \mu \mathrm{m})$. A nominal laser power of 1 watt peak, modulated 100 percent at $1 \mathrm{kc}$, was assumed for the calculation, with the following attenuations:

\begin{tabular}{lc} 
Laser chain & $\frac{\mathrm{JB}}{45}$ \\
Polarizer/beamsplitter & 17 \\
Sensor internal beamsplitters & 6 \\
Detector a perture $\left(29 \sigma_{0}\right)$ & 6 \\
Modulation (peak to rms) & 4.5 \\
Miscellanecus losses & 1.5 \\
\hline Total attenuation & $80 \mathrm{~dB}$
\end{tabular}

Therefore total laser power, $P_{\text {sig' }}$ to the detector is 10 nwalts and:

$$
S / N=\frac{P_{\text {sig }}}{X E P}=\frac{10^{-8}}{10^{-10}}=100
$$


Table 3-2, Focus parameter summary

Equation for Modulation Index

$$
M I=\frac{1}{\xi}\left[\frac{Z_{0} / \xi}{\left[1+\left(Z_{0} / \xi\right)^{2}\right]^{2}}\right] \frac{\left(\mathrm{r} / \sigma_{0}\right)}{\operatorname{ExP} \frac{\left(\mathrm{r} / \sigma_{0}\right)}{2\left[1+\left(Z_{0} / \xi\right)^{2}\right]}-1}
$$

Where:

$$
\begin{aligned}
\sigma_{0} & =\text { beam radius }(1 / e) \\
\xi & =\text { depth of focus } \\
r & =\text { detector radius } \\
Z_{0} & =\text { detector spacing }
\end{aligned}
$$

Theoretical Values

\begin{tabular}{|c|c|c|c|}
\hline & Equation $^{*}$ & Al Target & In Ser:sor \\
\hline$\xi$ & $2 N \lambda\left(f / \mathrm{no}_{0}\right)^{2}$ & $13 \mu \mathrm{m}$ & $5.3 \mathrm{~cm}$ \\
$\sigma_{0}$ & $\frac{N \lambda}{2}\left(f / \mathrm{no}_{0}\right)$ & $2.1 \mu \mathrm{m}$ & $133 \mu \mathrm{m}$ \\
$Z_{0}$ & $2 \xi$ & $26 \mu \mathrm{m}$ & $10.3 \mathrm{~cm}$ \\
$r$ & $\sigma_{0}$ & $2 \mu \mathrm{m}$ & $133 \mu \mathrm{m}$ \\
\hline
\end{tabular}

\section{Theoretical Results}

$$
\begin{array}{ll}
\text { Modulation index (MI) } & 5.7 \% / \mu \mathrm{m} \\
\text { Portion of total energy } & 29 \%
\end{array}
$$

* $N=2,5$

f/nc. at target $=1.58$

i/no. at sensor $=100$

Equation based on gaussian illumination function truncated at $1 / \mathrm{e}^{2}$. 
Focus error, c, due to the detector nuise is given by:

$$
=\frac{1}{(\mathrm{MI})(\mathrm{S} / \mathrm{N})}=0.17 \mu \mathrm{m}
$$

This is small enough to ensure that detector noise will not be of a concern in the actual system. This is especially true since the most inexponsive detector with amplifier was chosen for the calculation.

\section{Focus and Pointing Implementation}

\section{Focus Sensing}

There are several ways in which the iocus sensing technique die scribed in the previous subsection can br implemented. The scheme used is shown in Figure 3-7. There are two optical patis to the detector. The upper path, path A, comes to a focus at point A past the detector. The lower path. path $B$, which is folded down by the beamsplitter to the two mirrors, and then back up toward the detector. comes to a focus in front of the detector.

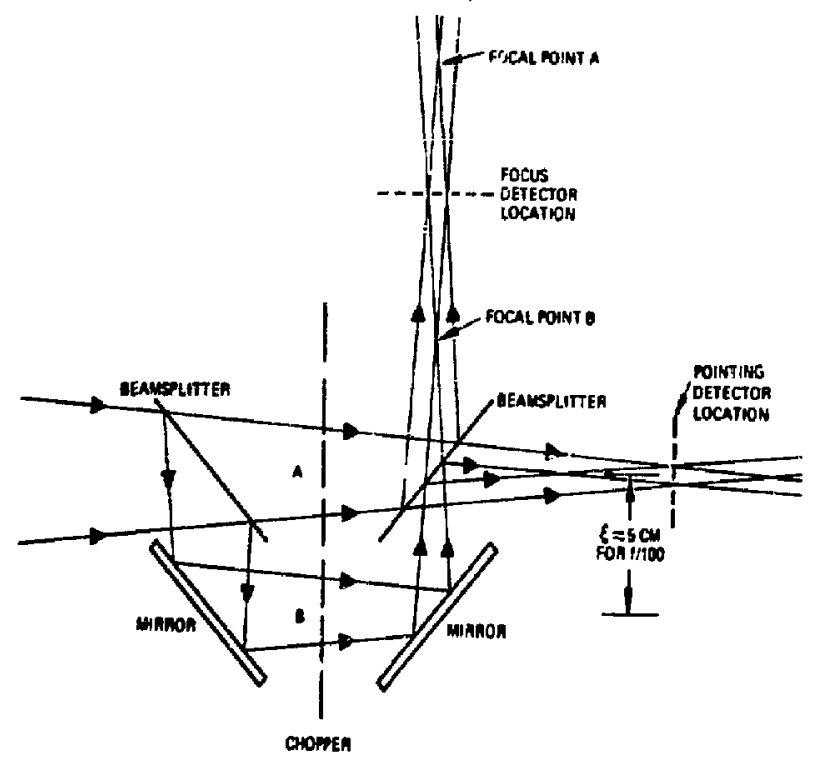

Figure 3-7. Selected focus delection scheme utilizing single doctector and chopper 
The detector may be thought of as being located before focal point $A$ and after focal point B, the same as the two detectors in Figure 3-3. By alternately switching betwe?n the two paths with a mechanical chopping wheel, the modulation is generated in a single detectur rather than utilizing two detectors. This approach eliminates the need for matching detectors and electronics. Due to the rather small MI of $5.70_{0} / \mu \mathrm{m}$, the two detec ir implementation would require that the detector 3 and electronics be matcinti to better than 5 percent and it would require frequent testing to ensure thas remained matched. Although 5 percent is not an unreasonable figure, matching for 20 or 32 channels would be at best a nuisance. Aiso two detectors would require apertur. :iameter matching to better than 2 perces, otherwise variations in laser power would cause an offset.

\section{Pointing Sensing}

Poirting sersing is implemented with a conventioual quad detector. As shown in Figure $3-7$, the pointing detector has the same equivalent location as the focus detector, i.e., it is located so that the ene-gy is focused alternately beiore and after the detectors. This has two advantages. The first advantage is that the pointing detector is averaging the location of the centroid of energy before and after the focal point so that the affective aberrations are minimized, and the pointing is accomplished with exactly the same information as the focus. Figure 3.8 shows how large amounts of coma affect the location of the centroid as a function of focal distanze. Since the spacing between focal points and detector is twice the depth of focus $\left(Z_{\mathbf{0}}=2 \xi\right)$, the major portion of the centroid shift is eliminated. In Figure 3-8, the ser.sing point is off the graph since $2 \xi=26 \mathrm{~m}$. It can be seen that the curve is approaching the axis al that point which means that the erro: due to aberrations is minirnized. The second advantage which accrues due to locating the detector in this manner, is that the average blur diametre is increased since the detector is out of focus by effectively twice the depth of focus. Further, the blur diameter will not appreciably change it the laser chain wers 10 be either diffraction limited or worse than 2.5. If the detector were utilized at the focus, then the focused spot would largely be lost in the crack (separation between elements). If the detector were to simply be delocused, then there would be a null shift duc to non-symmetrical aberration as shown in the Figure 3.8 . 


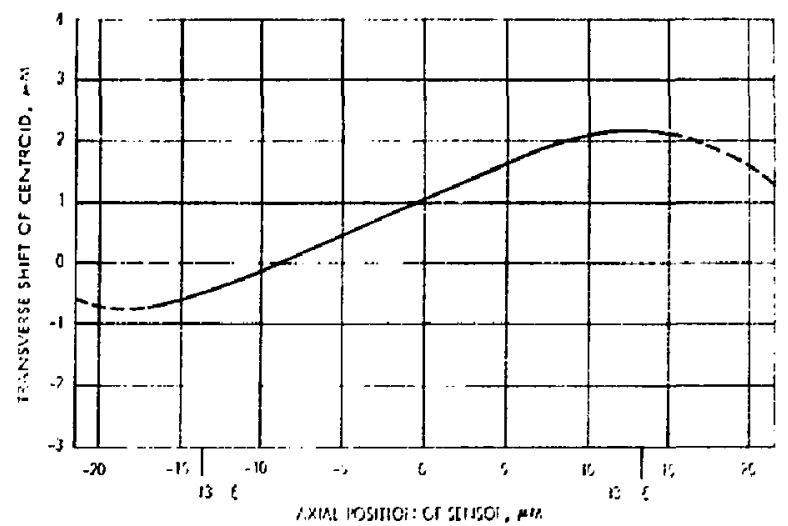

Figure 3-8. Centroid shift as a function of focus with one wave: of coma

The $1 / \mathrm{e}$ radius of the effcetive blur at the detector is $\sqrt{5} \mathrm{x} \sigma_{0}=300 \mu \mathrm{m}$ or the $1 / \mathrm{e}$ dianeter is $600 \mu \mathrm{m}$. This is large enough so that the separation between elements does not significantly affect operation of the quad. For a quad the MI is given by

$$
\text { MI }=\sqrt{\frac{2}{\pi}} \frac{1}{\sigma_{0}}=\sqrt{\frac{2}{5 \pi}} \frac{1}{\sigma_{0}}
$$

Where:

$\sigma=1 /$ rafius on detector

As a conservative approach an inexpensive diffused junction, off the shelf, detector array was chosen and the design executed with this detector. This approach ensures the minimum cost and at the same time provides a backup position for going to a better (and more expensive) detector in the event of an unforeseen problem. The detector selected is a UDT PIN SPOT/4i having the following pararneters of interest:

Active area

Element separation

$\operatorname{NEP}(W, 1 \mathrm{~Hz}, 1.06 \mu \mathrm{m})$
$2.5 \mathrm{~mm} \times 2.5 \mathrm{~mm}$

$0.125 \mathrm{~mm}$

$10^{-11} \mathrm{~W}$ 
Using the same parameters as in the focus sensor calculation gives a pointing MI, referred to the target, as follows:

$$
M I=170 \% / \mu \mathrm{m}
$$

and a signal to noise ratio, $S / N$, for the quad as follows:

$$
\mathrm{S} / \mathrm{N}=\frac{\mathrm{P}_{\mathrm{sig}}}{\sqrt{4 \mathrm{NEP}}}=2.5 \times 10^{3}
$$

The $\sqrt{4}$ comes in because there are four detector elements. The pointing error due to detector noise is given by:

$$
\epsilon=\frac{1}{(\mathrm{MI}) \mathrm{S} / \mathrm{N}}=2.4 \times 10^{-3} \mu \mathrm{m}
$$

As with the focus detectors, the magnitude of the noise error indic ates that it will be insignificant in the actual system. Balance between the detector elements and electronics will have to be held to better than 17 percent which is no problem.

Pointing and focusing sensor design parameters are summarized in Table 3-3 and performance predictions in Table 3-4 and 3-5.

\section{Hardware Layout}

Figures 3-9 and 3-10 show a layout and pictorial view of the pointing and focus sensor. The beamsplitter and folding assembly is fabricated from three identical beamsplitting cubes. The second lens and the beam splitter are in optical contact to eliminate reflections.

\section{Modulator (Focus) Disk Sizing and Location Analysis}

It is highly cesirable from a cost savings standpoint to provide as $t$. thy functions from a given piece of hardware as possible. Therefore, the single modulator dis' is designed to provide a $50 \%$ duty cycle for both optical paths and to modulate both paths on and off identically, considering energy transmitted to detector, as a functio: of time. If we allow the two different beam diameters at the glass exits to subtend the same angle from the disk center. each beam path will be modulated on and off identically for a constant spred 
Table 3-3. Pointing and focusing design parameters

$\begin{array}{ll}\text { Laser } & \\ \text { Power } & 1 \text { watt cw } \\ \text { Modulation } & 106 \% \text { at } 1 \mathrm{kHz} \\ \text { Amplitude stability } & 1 \% \\ \text { Attenuation - laser to detector } & 80 \mathrm{~dB} \\ \text { Sensor } & \\ \text { Aperture diameler } & 80 \mathrm{~mm} \\ \text { f/No. } & \$ 100 \\ \text { Focal point modulation } & 10 \mathrm{~cm} \\ \text { Acquisition field (l mm target) } & \pm 28 \mu \mathrm{m} \text { (at target) } \\ \text { Acquisition field (7 mm target) } & \pm 140 \mu \mathrm{m} \text { (at target) } \\ \text { Focus Detector } & \\ \text { Type (with op am } \mathrm{p} \text { ) } & \mathrm{UDT}-400 \\ \text { NEP (1.06 } \mu \mathrm{m}, 1 \mathrm{kHz}, 1 \mathrm{~Hz}) & 10^{-10} \mathrm{~W} \\ \text { Active diameter } & 2.5 \mathrm{~mm} \\ \text { Aperture } & 270 \mu \mathrm{m} \\ \text { Price } & \$ 65 \\ \text { Pointing Detector } & \\ \text { Type } & \end{array}$


Table 3-4. Point and focus sensor performance

\begin{tabular}{|lll|}
\hline & \multicolumn{1}{c}{ Focus } & \multicolumn{1}{c|}{ Pointing } \\
$\mathrm{MI}$ & $5.7 \% / \mu \mathrm{m}$ & $17 \% / \mu \mathrm{m}$ \\
$\mathrm{S} / \mathrm{N}$ & $10^{2}$ & $2.5 \times 10^{3}$ \\
Calc Er ror & $0.17 \mu \mathrm{m}$ & $2.4 \times 10^{-3} \mu \mathrm{m}$ \\
Required balance & $5.7 \% / \mu \mathrm{m}$ & $17 \% / \mu \mathrm{m}$ \\
Acquisition range & & \\
7 mm target & $\pm 500 \mu \mathrm{m} /$ axis & $\pm 100 \mu \mathrm{m} / \mathrm{axis}$ \\
$1 \mathrm{~mm}$ target & $\pm 100 \mu \mathrm{m} /$ axis & $\pm 28 \mu \mathrm{m} / \mathrm{axis}$ \\
\hline
\end{tabular}

of the modulator disk. The modulator disk intercepts one optical path after 10 inches of glass travel and intercepts the second path after 11.5 inches of glass travel. Calculation of beam diameters at modulator disk:

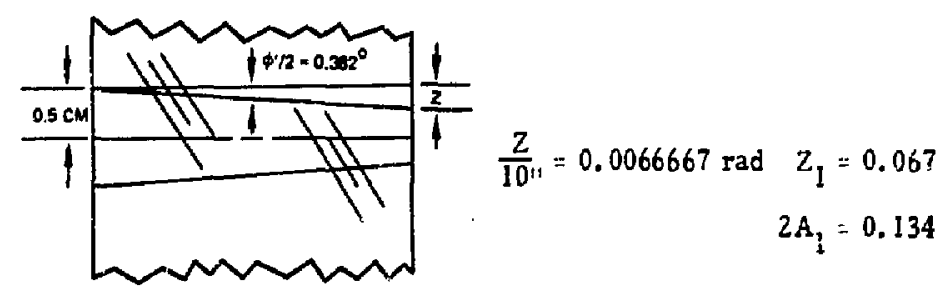

$\therefore$ beam diameter after 10 inches of glass travel $\cong 0.394$ inches $0.135 \cong 0.260$ inches $=d_{2}$

$\therefore$ beam diameter after $11.5 \pm$ of glass travel $Z_{2} / 11.5=0.006666$ $\mathrm{z}_{2}=0.0767 \quad 2 \mathrm{z}_{2}=0.153 \quad 0.394-0.153 \stackrel{2}{=} 0.241=\mathrm{d}_{\mathrm{I}}$ $\therefore$ The beam diat: eter ratio $\cong \frac{0.241}{0.260}=0.927$ (to be used iater in 
Table 3-5. Alignment sensitivity for pointing and focus sensor

\begin{tabular}{|c|l|l|l|}
\hline & \multicolumn{1}{|c|}{ Transverse } & \multicolumn{1}{|c|}{ Angular } & \multicolumn{1}{|c|}{ Axial } \\
\hline Sensitivity & $0.84 \mu \mathrm{m} / \mathrm{mm}$ & $1.5 \times 10^{-2} \mu \mathrm{m} / \mathrm{urad}$ & $0.7 \mu \mathrm{m} / \mathrm{m}$ \\
Error $/ \mu \mathrm{m}$ & $1.2 \mathrm{~mm}$ & $65 \mu \mathrm{rad}$ & $1.4 \mathrm{~m}$ \\
\hline
\end{tabular}




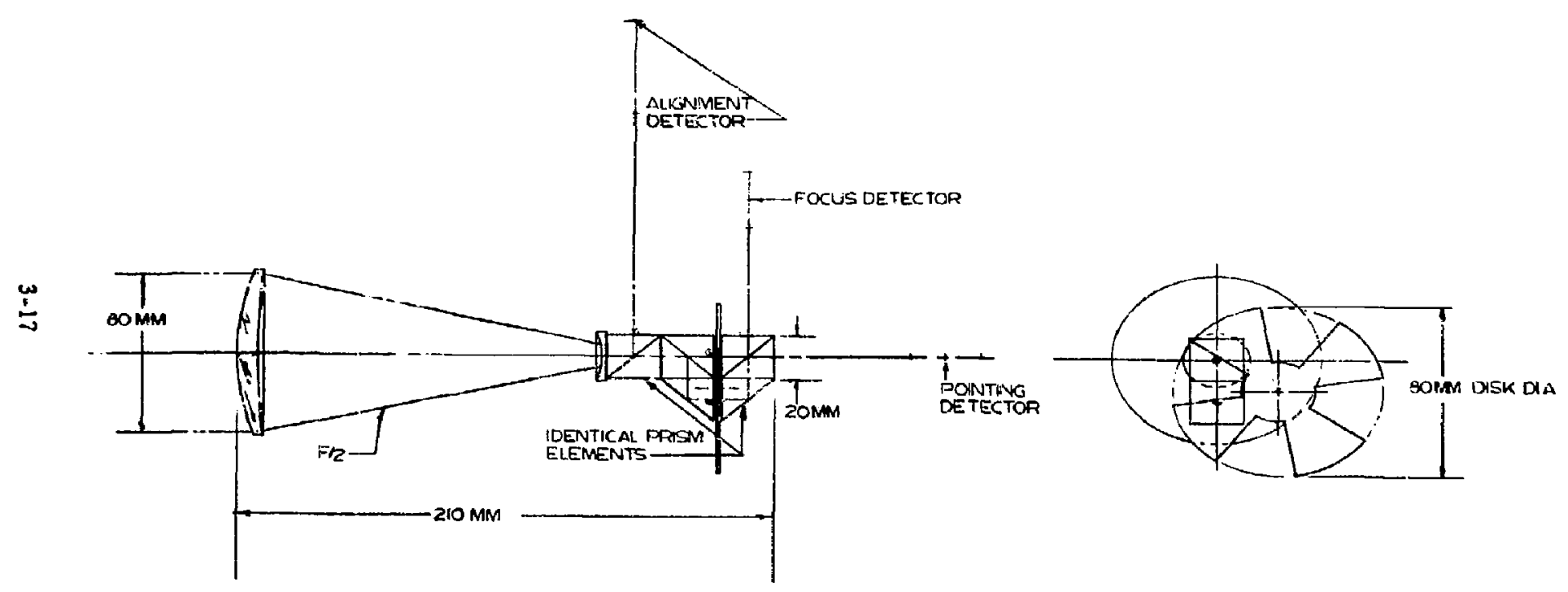

Figure 3-9. Pointing and focus sensor layout 


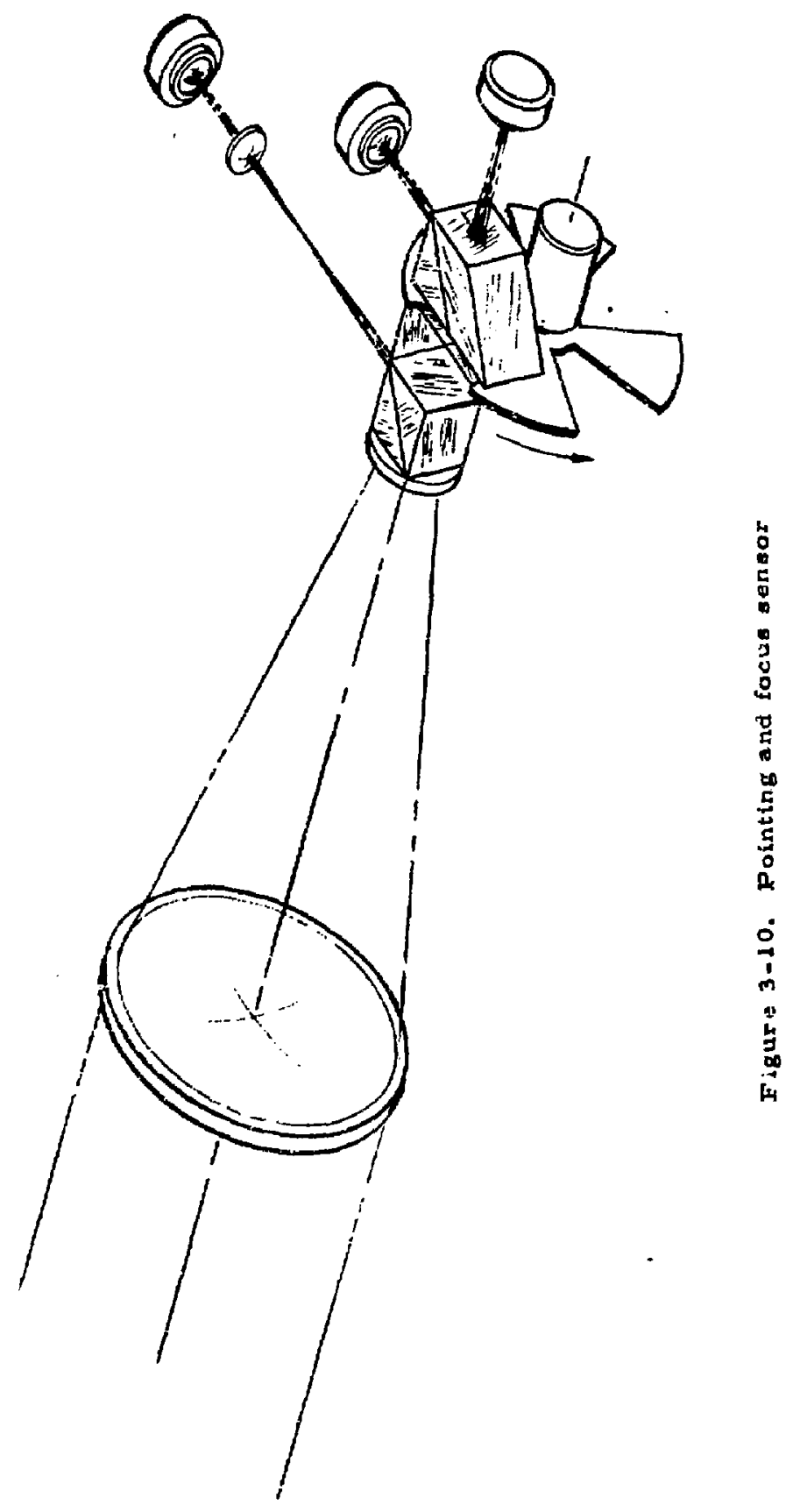




\section{Disk Sizing}

The following assumptions are made: Assuming

1. $50 \%$ duty cycle, the refore an even integer number of spaces and slots

2. from previous calculations and consideraticns of jepth of focus, the distance between beam centers it known to be 1.5 inches

3. the sum of the signals for an in focus condition ecuals a constant.

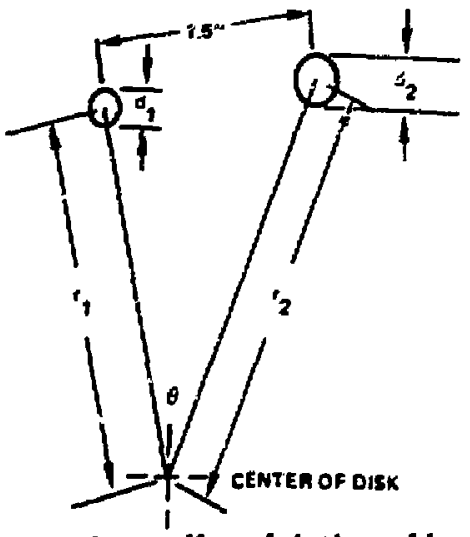

$$
\begin{aligned}
& d_{1}=\text { diameter of beam after } \\
& d_{2}=\text { diameter of beam afier }
\end{aligned}
$$

For equal on-off modulation of both beams:

$$
\begin{aligned}
& \frac{\frac{d_{1}}{r_{1}}}{r_{1}}=\sin \phi \\
& \frac{\frac{d_{2}}{2}}{2}=\sin \phi \\
& \therefore \frac{d}{r_{1}}=\frac{d_{2}}{r_{2}} \text { and } \frac{r_{1}}{r_{2}}=\frac{d_{1}}{d_{2}} \\
& \text { and } \theta=\theta+\phi-\phi
\end{aligned}
$$

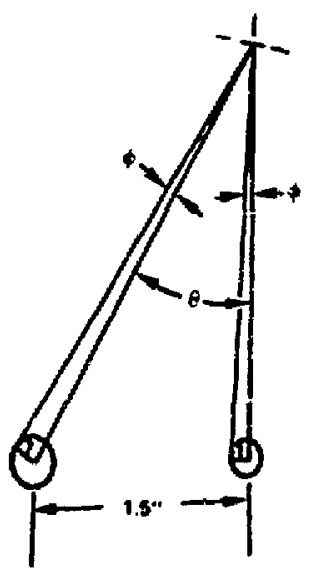

Therefore the slot angulat subtense in the modulator disk is equal to the angle between the radii to the beam centers. If the disk is $c$ ided into eight 
equal sections, four spaces and four blanking segments, then $\theta=\frac{360^{\circ}}{8}=45^{\circ}$. Now the triangle is defined, and only one value for $r_{1}$ and $r_{2}$ will satisfy the equations for an oblique triangle with a given value for $r_{1} / r_{2}$.

Defining Components of the triangle

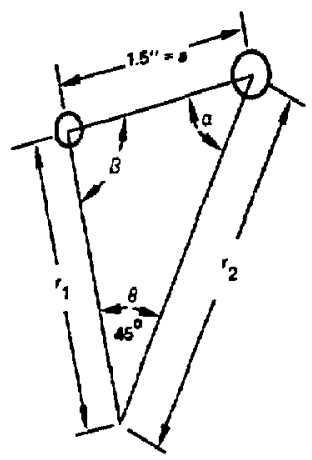

Equations for the oblique triangle

$$
\sin \alpha=\frac{r_{1} \sin \theta}{a}=\frac{r_{1} \sin 45^{\circ}}{1.5} ; \alpha=\sin ^{-1}\left(\frac{r_{1} \sin 45^{\circ}}{1.5}\right)
$$

$$
\begin{aligned}
& \beta=180^{\circ}-\theta-a \\
& \mathbf{r}_{2}=\frac{1,5 \sin \beta}{\sin +5^{\circ}}
\end{aligned}
$$

and

$$
\frac{r_{1}}{r_{2}}=\frac{d_{1}}{d_{2}}=\frac{0.241}{0.260}=0.9269 \text { must be satisfied. }
$$

By assigning values to $r_{1}$ and solving for $r_{2}$, as shown in the table below, solve the triangle for the appropriate radii.

\begin{tabular}{c|c|c|c|c|c|c|c|c|} 
Attempt & $\mathrm{r}_{2}$ & $\mathrm{r}_{1}$ & $\theta$ & $\mathrm{a}$ & $\mathrm{d}_{1} / \mathrm{d}_{2}$ & $\mathrm{r}_{1} / \mathrm{r}_{2}$ & $\alpha$ & \multicolumn{1}{c}{$\beta$} \\
\hline 1 & 2.067 & 1.8 & $45^{\circ}$ & 1.5 & 0.927 & 0.871 & $58^{\circ}$ & $77^{\circ}$ \\
2 & 2.031 & 1.87 & $45^{\circ}$ & 1.5 & 0.927 & 0.921 & $61.81^{\circ}$ & $73.19^{\circ}$ \\
3 & 2.025 & 1.88 & $45^{\circ}$ & 1.5 & 0.927 & 0.928 & $62.39^{\circ}$ & $72.61^{\circ}$
\end{tabular}


Attempted Detail Calculations:

1. $\begin{aligned} \alpha & =\sin ^{-1}\left(\frac{1.8 \times 0.707}{1.5}\right)=\sin ^{-1} 0.8484 \alpha=58^{\circ} . \\ \beta & =180-45-58=180-103=77^{\circ} \sin \beta=0.9744 \\ r_{2} & =\frac{1.5 \times 0.9744}{0.707}=2.067 \frac{r_{1}}{r_{2}}=\frac{1.8}{2.067}=0.871\end{aligned}$

2. $\alpha=\sin ^{-1}\left(\frac{1.87 \times 0.707}{1.5}\right)=\sin ^{-1} 0.88139 a=\frac{61.81^{\circ}}{\frac{45}{106.81^{\circ}}}$

$\beta=180-106.81=73.19^{\circ} \sin \beta=0.9573$

$r_{2}=\frac{1.5 \times 0.9573}{0.707}=2.031 \quad \frac{r_{1}}{r_{2}}=\frac{1.87}{2.031}=0.921$

3. $\alpha=\sin ^{-1}\left(\frac{1.88 \times 0.707}{1.5}\right)=\sin ^{-1} 0.8861 \alpha=\frac{62.39^{\circ}}{\frac{45}{107.39^{\circ}}}$

$\beta=180-107.39=72.61^{\circ} \sin \beta=0.9543$

$r_{2}=\frac{1.5 \times 0.9543}{0.707}=2.0247 \frac{r_{1}}{r_{2}}=0.9285$

By knowing the radii and the location of the beam centers, it is a simple matter to locate the appropriate center of rotation for the modulator disk. The scale optical layout indicates the center location of the disk. The disk need $\varepsilon$ to be about five inches in diameter to cover a one inch undefined (initial) location of the beam centers. Plus or minus a half inch in undefined location is required so that system tolerances may be accommodated.

Also by knowing the radii, calculate the ratio of beam un-time to beam turn-on time, i.e., it is assumed that it takes a dimension of one beam diameter to fully turn the beam on as the modulator disk blade pas ses through the beam. For eight segments in the blade:

$\frac{2 \pi \times 1.88}{8}=1.476$ circumferential inches 
The beam diameter at this radius equals 0.241 inch, therefore the ratio of modulator blade width to beam diameter is: $1.476 / 0.241=6.12$.

For longer on-off periods with respect to the time to turn on, i. e., to pass one beam diameter, a larger angle than $45^{\circ}$ must be chosen. However, the new angle must be an even integer, i. e., $360^{\circ} / 6,360^{\circ} / 4$ and $360^{\circ} / 2$ would be appropriate. 


\section{THE FOCUSING LENS CENTERING SENSOR}

\section{RERUIREMENTS}

The basic requirements (from Table 2-6) for the centering sensor are:

Null Stability

Noise
$120 \mu \mathrm{m}$

$<20 \mu \mathrm{m}$

\section{SENSING TECHNIQUE AND DESCRIPTION}

The technique for sensing the position of the beam is similar to a conventional quad, except that the four elements are provided with individual optics and separated so that they are at the edge of the beam. The arrangement is shown in Figure 4-1. The sensors index out of the beam when not in use. Ail sensors on Shiva have shutters that closes when sensor power is removed. In the case of the centering sensor, the sensor indexes back behind its stationary shutter when power is removed. The alignment laser beam has been truncated at $22 \mathrm{~cm}$ to provide a well defined edge for the centering sensors, as shown on the left side of the figure. The beam is truncated so that the edge is almost exactiy in the center of the sensor so that the aperture is one half illuminated. The sensor function as an energy bucket and the signal from opposing sensors is balanced one against another as in a quad. The electronics are in fact, more simple than a quad and individual detectors can be purchased with op-amps on the same substrate. Quads are not available with built in amplifiers.

Figure 4-2 shows the sensor design. The field lens acts to reimage the entrance aperture on the detector so that the sensor is not sensitive to angle. A very low quality field lens is used since it is desiraible to diffuse the energy on the detector. Parameters have been selected so that there are virtually no internal alignment requirements.

\section{PREDICTED PERFORMANCE}

MI was computed by assuming a well defined beam having an edge at $22 \mathrm{~cm}$ with the sensor centered on the edge. The parabolic intensity profile was as sumed. 


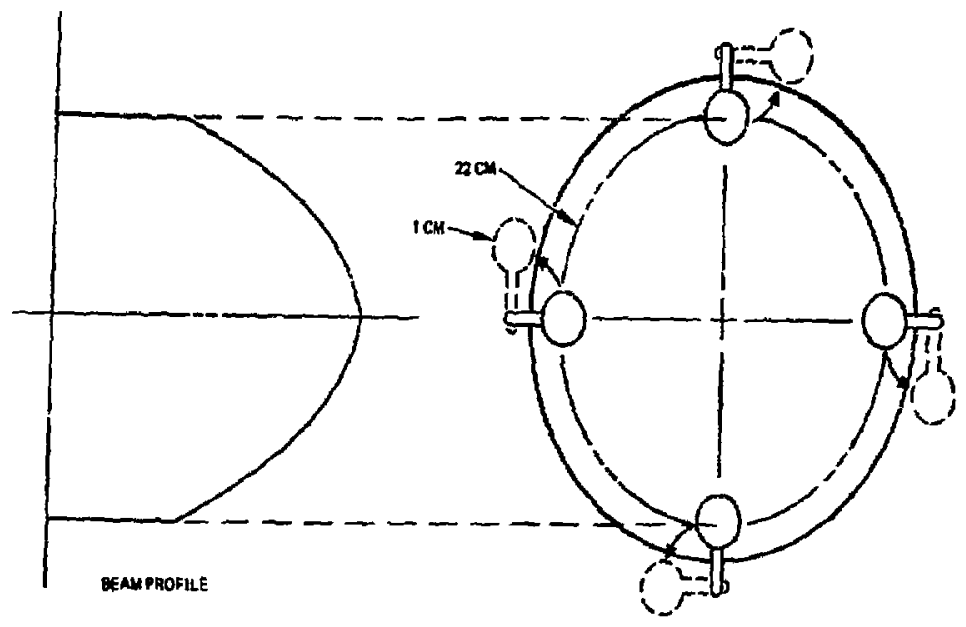

Figure 4-1. Centering sensor configuration

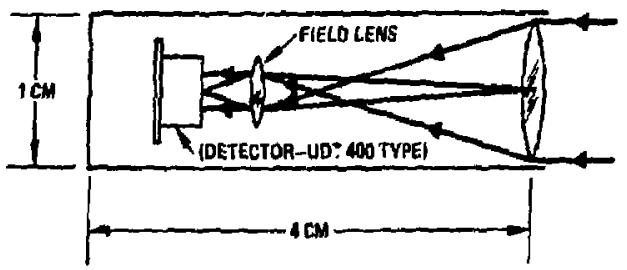

Figure 4-2. Final lens cente ring sensor, four/channels

Table 4-l gives the predicted performance. Three percent is a reasonable balance to maintain between channels, although a looser tolerance would be more desirable. The MI can be improved by masking part of the aperture so that the average signal level is reduced. This would help the balance tolerance but would do nothing for the calculated noise. The predicted performance, as shown, is acceptable. 
Table 4-1, Centering sensors

\begin{tabular}{|l|l|}
\hline & Final Lens \\
\hline Aperture diameter & $1 \mathrm{~cm}$ \\
\hline Detector type & $\begin{array}{l}\text { Single element } \\
(\mathrm{UDT}-400)\end{array}$ \\
\hline Modulation index & $0.025 \% / \mu \mathrm{M}$ \\
\hline $\begin{array}{l}\text { Galeulate detector noise } \\
\text { (1 pratt align laser) }\end{array}$ & $17 \mu \mathrm{M}$ \\
\hline $\begin{array}{l}\text { Channel balance } \\
\text { (for } 120 \mu \mathrm{m} \text { stability) }\end{array}$ & $3 \%$ \\
\hline Sensors/laser chain & 4 \\
\hline
\end{tabular}




\section{PINHOLE SENSOR}

\section{REQUIREMENTS}

The basic requirements for the pinhole sensor come from Tables 2-3 and $2-4$ in Section 2. Since the given requirements are for total error they must be divided by $\sqrt{2}$ to get per axis error. The error requirements become $80 \mu \mathrm{m}$ centering error per axis and $5 \mathrm{\mu rad}$ pointing error per axis. The sensor has been devigned so that the total error can be allocated to mechanical tolerancee.

\section{SENSING TECHNIQUE AND FHYSICAL DESCRIPTION}

For pointing, a quad is utilized in the conventional configuration, and for centering, a quad is utilized by reimaging a plane in the beam near the entrance aperture of the pinhole. The optical configuration is shown in Figures 5-1 and 5-2. The sensor input lens is identical to the input lens for the pinhole and is mounted rigidly beside it so that the focal point of the sensor is beside the pinhole. The beamsplitter inside the sensor, shown in Figure 5-2, picks up the energy just prior tc. locus so that the lens (cemented to the back of the beamsplitter) reimages the entrance aperture on tha centering quad. The other half of the energy is tranamitted by the beamsplitter to the pointing quad which is mounted directly to the beamspiitter.

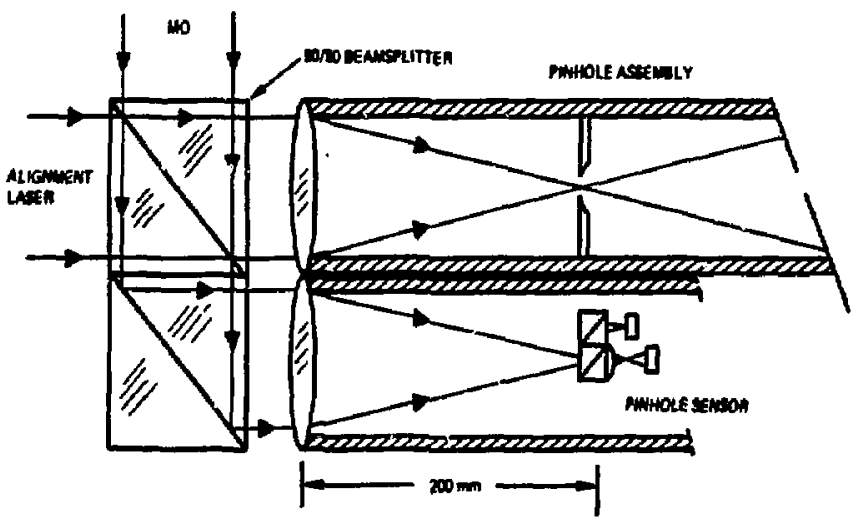

Figure 5-1. Pinhole sensor configuration 


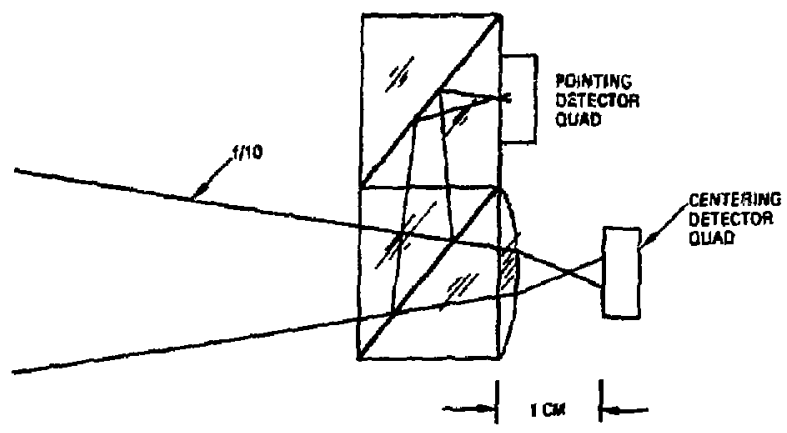

Tigure 5-2. Pinhole sensor

Focal lengths have been selected so that the image on the $2.5 \mathrm{~mm}$ centering quad is about $1 \mathrm{~mm}$. The image on the pointing quad is the same size as the image on the pinhole (the $1 / \mathrm{e}^{2}$ diameter is oniy $22 \mu \mathrm{m}$ ) therefore a detector with no separation between elements must be used. The method used by the manufacturer for eliminating the dead zone is described in Appendix $I$.

The objective in the layout was to choose and mount components so that alignment would be very stable. Towards this end the focal point of the sensor has been located directly adjacent to the pinhole structure so that alignment between the two is preserved even if the pinhole structure deflects. The input beamsplitter is also critical to alignment so a pair of cemented cubes are used to insure that the beam into the sensor is always referenced to the beam into the pinhole lens.

The beamsplitter method of combining beams, described above, is somewhat wasteful of energy from both the master oscillator and the alignment laser in that only 50 percent is made available for use. By recourse to polarization phenomena, an alternate method of combining beams, described here, makes a vailabie essentially all of the energy emitted by the MO and the AL. A basic assumption of this alternate technique is that the outpits of each laser is polarized.

Figure 5.3 illustrates the scheme. The part enclosed by the dashed line is identical to the components and methoo described above except that the beamsplitter block (A) is very low in reflectivity and very high in transmission. 


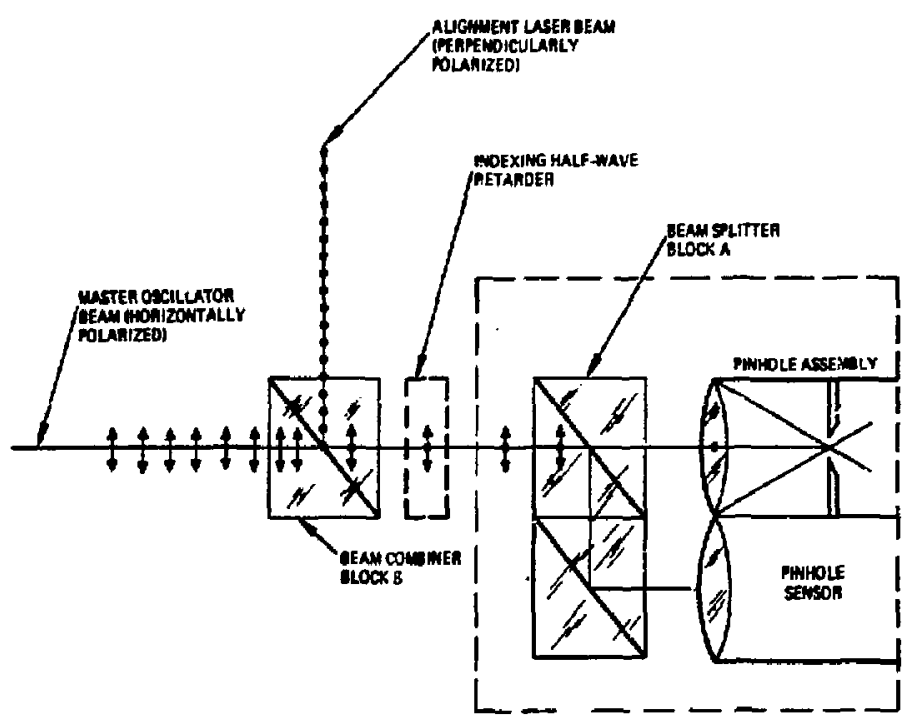

Figure 5-3. Alternate method of combining of master oscillator and alignment laser beams

As descriied in the preceding subsection, the beamsplitter, the sensor, and the pinhole assembly are gualified to one anothe $r$ at assembly.

The beam combiner block $B$ transmitts $\approx 100$ percent of the radiant energy incident upon its dielectric diagonal surface which is polarized parallel to the plane of incidence (parallel to the plane of the paper as shown). The diagonal surface reflects $=100$ percent of the incident energy which is polarized perpendicular to the plane of incidence. The output of the MO is arranged to be plane polarized parallel to the plane of incidence, as shown, and the AL output is made perpendicular. They enter the combiner block in the directions shown and are combined with essentially 100 percent efficiency.

The alignment beam electric vector orientation, after reflection from the beam combining surface, is improperly oriented to allow passage through the rest of the laser chain. The orientation is corrected by irserting a half-wave retarder plate in the beam path, as shown, whenever the AI. beam it in use. 
Wedge in the half-wave retarder is unimportant since it is always in the AL beam when being used either by the pinhole sensor or sensors further down stream. When the MO beam and the AI beam are aligned, aignment is done sequentially with the half-wave retarder being removed when the MO heam is in use.

\section{PERFORMANCE PREDICTIDNS}

The design parameters and precicted performance for the pinhole sensor are summarized in Table 5-1.

Table 5-1. Design parameters and performancs summary

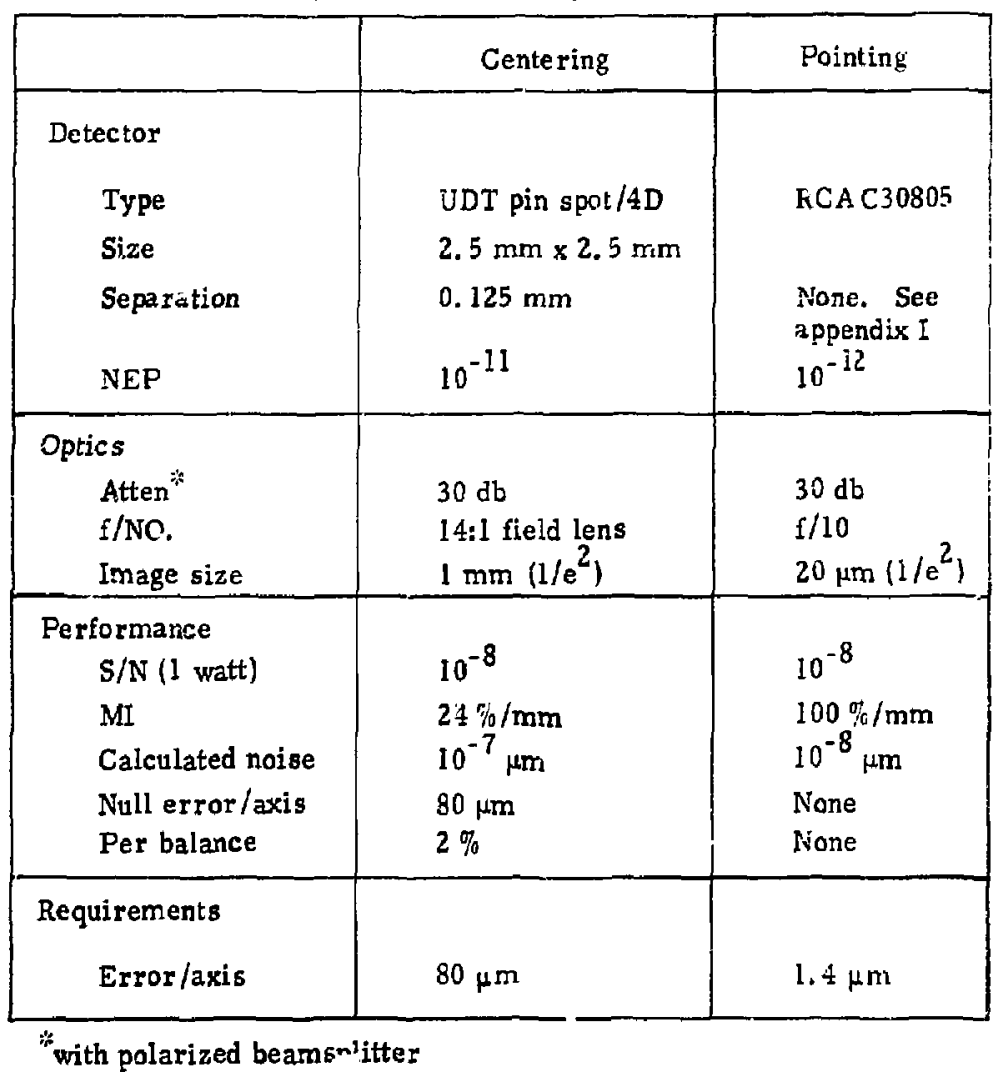




\section{LASER CHAIN CENTERING SENSORS}

\section{REQUIREMENTS}

The error budget requirements for the laser chain centering sensors are from Tables $2-7$ and $2-8$ in Section 2 , the ge are repeated in Table 6-1 on a per axis basis.

\section{APODIZE APERTURE SENSOR DESCSRIPTION}

The prime requirement is to insure that the sensor contributes no more than $7 \mu \mathrm{m}$ of error when centering the $18 \mathrm{~mm}$ beam in the apodized aperture. The only way that this can be accomplished is for the sensor to compare directly the $18 \mathrm{~mm}$ beam and the apodized aperture. This is accomplished by reimaging the apodized aperture on and the $18 \mathrm{~mm}$ ieam ori a quad detector as shown in Figures $6-1$ and $6-2$. When the indexirg aperture in cut, the apodized aperture is fully illuminated and the afodized profile is imaged on the quad. This is used to calibrate the sensor so that the beamsplitter alignment is corrected. When the $18 \mathrm{~mm}$ aperture is indexed in, then this is imaged on the quad and the FEP is controlled to null the sensor output. To meet the accuracy requirement, the sensor noise must be less than $5 \mu \mathrm{m}$. Since it is a comparison process, detector and signal processing null balance are not important unless there is a change during the measurement cycle.

Table 6-1. Frror allocation requirements

\begin{tabular}{|l|c|}
\hline & Per Axis Error \\
\hline Apodized aperture sensor & $7 \mu \mathrm{m}$ \\
Last spatial filter sensor & $500 \mu \mathrm{m}$ \\
\hline
\end{tabular}




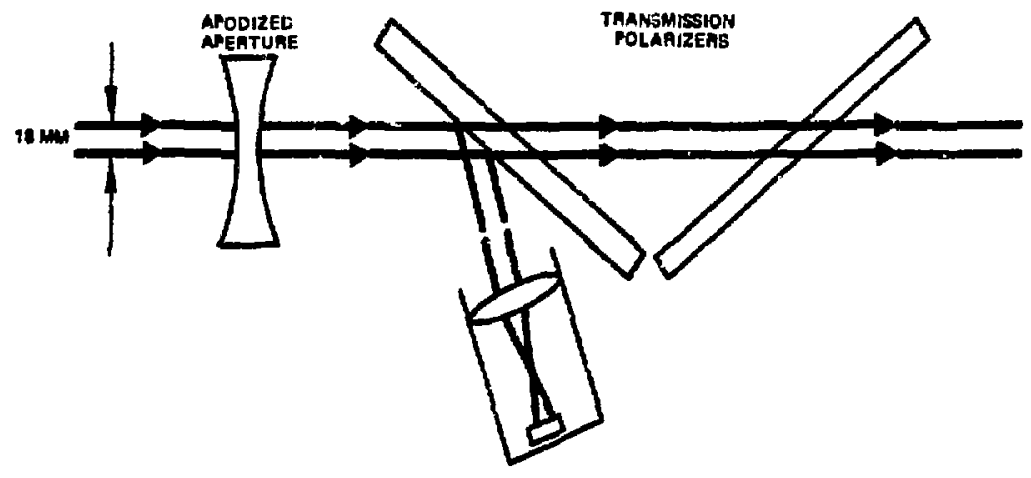

Figure 6-1. Apodized aperture sensor optical iayout 


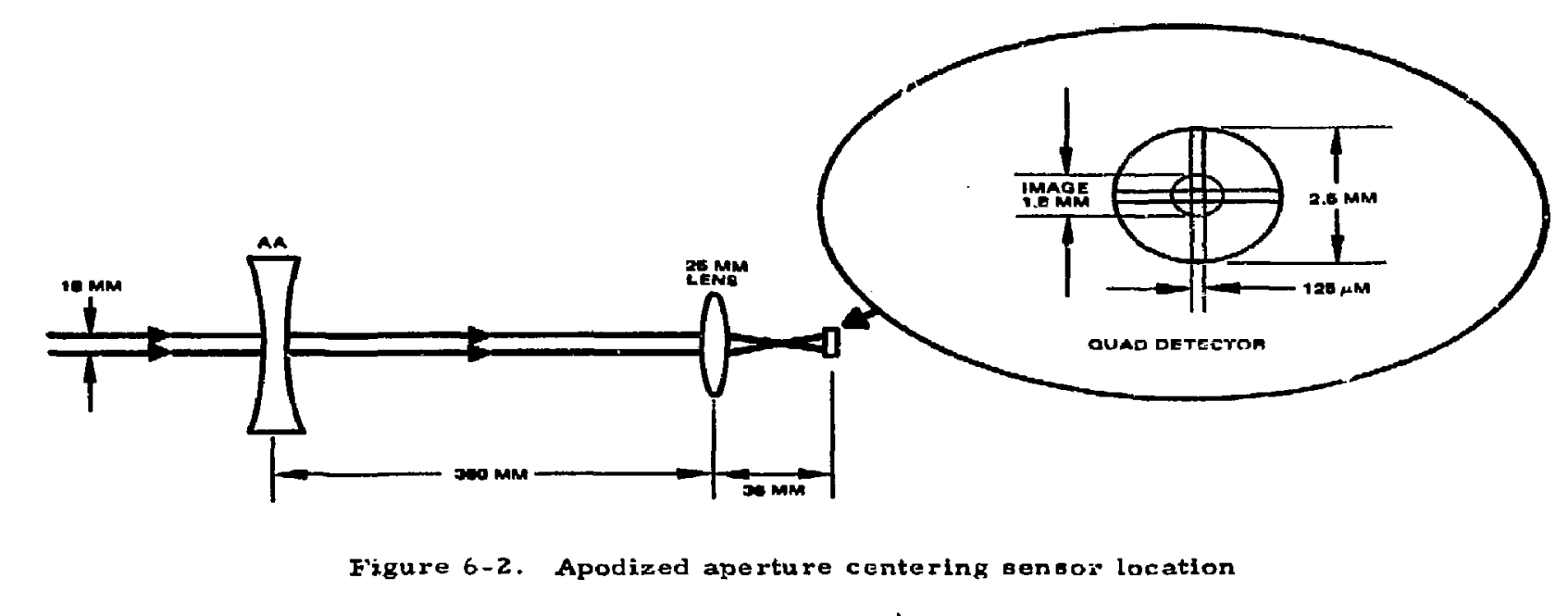


Alignment laser amplitude stability during the measurement eycle is also important. A conventionally used quad in the AA sensor would have a modulation index of only $0.04 \% / \mu \mathrm{m}$. The MI can be improved at least an order of magnitude by masking the center of the detector. Normally this would not be acceptable because of the loss in average signal level, however in this application it is feasible becsuse there is little attenuation to the laser power at the apodized aperture. This masking provides an MI of about $0.4 \% / \mu \mathrm{m}$ or a tota: modulation of 1 percent for $5 \mu \mathrm{m}$ error. This is still tight but a reasonable signal.

The alignment laser has an overall amplitude stability of 0.5 percent, but there is no information regarding the temporal characteristics of this 0.5 percent variation. Althougt the 0.5 percent falls within the sensor requirements, further improvement can be ob:ained by using AGC in the sensor error processing electronics.

\section{LAST SPATIAL FILTER SENSOR DESCRIPTION}

Figurt 6.3 shows the configuration of the sensor and its mount. The sensor lens is a $14 \mathrm{~cm}$ diameter $\mathrm{f} / 10$ lens. I ta field lens reimages the input aperture on a quad detector so that it measures the centering of the beam on the input lens. The input lens, the last reflective polarizer plate which is used as a beamgyitter and the folding mirror are mounted to the same mount as the input lens to provide positive registration. The angular stability between the last polarizer plate and the folding mirror is important in maintaining alignment between the sensor and the spatial filter. The sensor is designed to be insensitive to angle so that angular motion does not cause a centering error. The sensor is hard mounted in front so that the input lens is precisely registered with respect to the spatial filer lens but the rear mount can be softer.

As long as the sensor package does not bend, the sensor is as iong as the fi :st half of the spatial filter as that doesn't appear to be inconvenient and dises allow for inexpensive optics. Both lens in the sensor are minimum quality as there is nc appreciable imaging requirements. This configuration allows no significant errors due to mechanical tolerances. 


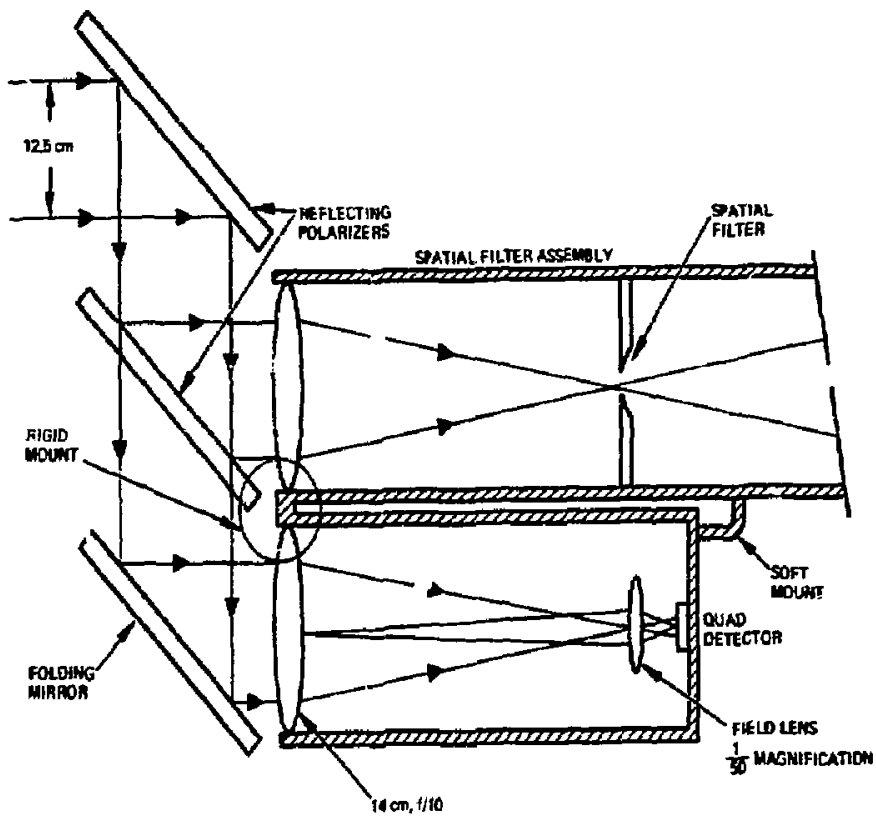

Figure 6-3. Last spatial filter centering sensor

The quad is $2.5 \mathrm{~mm} \times 2.5 \mathrm{~mm}$ and the entrance aperture image is about $2 \mathrm{~mm}$. The modulation index is about $3 \% / \mathrm{mm}$ which is adequate for $1.4 \mathrm{~mm}$ accurácy.

An interesting aside is the possibility of utilizing this sensor to also align the spatial filier so that the energy is centered in the spatial filter pinhole. The sensor would require a beamsplitter and another detector to provide the measurement. The sensor would then be mounted so that the detector package has positive registration with the pinhole as well as the input lens.

\section{PREDICTED PERFORMANCE}

The design parameters and predicted performance for the two centering sensor is given in Table $6=2$. 
Table 6. 2. Design parameters and performance summary

\begin{tabular}{|c|c|c|}
\hline & Apodized Aperture & Last Spatial Filter \\
\hline \multicolumn{3}{|l|}{ Detector } \\
\hline Type & \multicolumn{2}{|l|}{ UDT pin spot/4D } \\
\hline Size & \multicolumn{2}{|l|}{$2.5 \mathrm{~mm} \times 2.5 \mathrm{~mm}$} \\
\hline Separation & \multirow{2}{*}{\multicolumn{2}{|c|}{$\begin{array}{l}125 \mu \mathrm{m} \\
10^{-11} \mathrm{w}\end{array}$}} \\
\hline $\operatorname{NEP}(1.06,1 \mathrm{~Hz})$ & & \\
\hline \multicolumn{3}{|l|}{ Optics } \\
\hline Atten (total) & $40 \mathrm{db}$ & $60 \mathrm{db}$ \\
\hline Input $f / \#$ & $\mathrm{f} / 10$ & $f / 10$ \\
\hline Image size & $2 \mathrm{~mm}$ & $2 \mathrm{~mm}$ \\
\hline \multicolumn{3}{|l|}{ Performance } \\
\hline$S / N(1 w)$ & $10^{45}$ & $10^{+} 3$ \\
\hline MI & $0.4 \% / \mu \mathrm{m}$ & $3 \% / \mathrm{mm}$ \\
\hline Calculated noise & $2 \times 10^{-3} \mu \mathrm{m}$ & $2 \times 10^{-1} \mu \mathrm{m}$ \\
\hline Null error/axis & NA & $1.5 \%$ \\
\hline Per balance & & $500 \mu \mathrm{m}$ \\
\hline Required accuracy/axis & $7 \mu \mathrm{m}$ & $500^{\circ} \mu \mathrm{m}$ \\
\hline
\end{tabular}




\section{PATHLENGTH SENSOR}

\section{PATHLENGTH EQUALIZATION}

The 100 psec $(3 \mathrm{~cm})$ pulse generated at the master oscillator is split and sent down the 20 amplifier chains of the Shiva laser. The resulting 20 pulses must arrive at the target simultaneously with an accuracy of a small fraction of this pulse width. Some sort of fringe counting technique like those used in current interferometers is not applicable because the chains a re physically seperate and because path length ambiguity is far in excess of a single fringe. This section describes a technique with which the required accuracy is obtainet by measuring the differential times of propagation of a mode locked laser pulse down the 20 channels.

\section{PROPOSED INSTRUMENTATION}

The proposed configuration is shown in Figure 7-1. The CW mode locked Nd:YAG alignment laser output is sent through the quiescent amplifier chains and reflected from a surrogate target sphere. The pulse train returns to a beamsplitter (BSI) and is directed to a heterodyned detector. The local oscillator beam is obtained via a frequency shifter ${ }^{*}$ and retro (cat's eye). The rotating wedge generates a sinusoidal variation of the optical length of the local oscillator arm to aid in the signal detection, as described below. The optical length of the $L O$ arm is $X_{o}$, and the optical length of the amplifier chains is $X_{n}(n=1, \ldots 20)$.

The principle of operation is illustrated in Figure 7-2. The laser output is a train of pulses of width $C T(\approx 60 \mathrm{~mm})$ and spacing $C T(x)$ meter $)$. If the LO and signal pulse trains arrive simul,taneously, then mixing occurs, and the difference frequency $\omega_{L O}$ will be present at the detector output. As the differerce in length of the $\mathrm{LO}$ and signal arms deviates from an integral multiple of pulse separations $\mathrm{mCT}$, then the two pulse trains no longer coincide, and the IF signal at $\omega_{L O}$ goes to zero. Without the rotating wedge and phase lock amplifier, the output signal would vary as the curve $v_{0}$, and path length

*Applied Physics Letters, p, 46, 1 October 1962. 


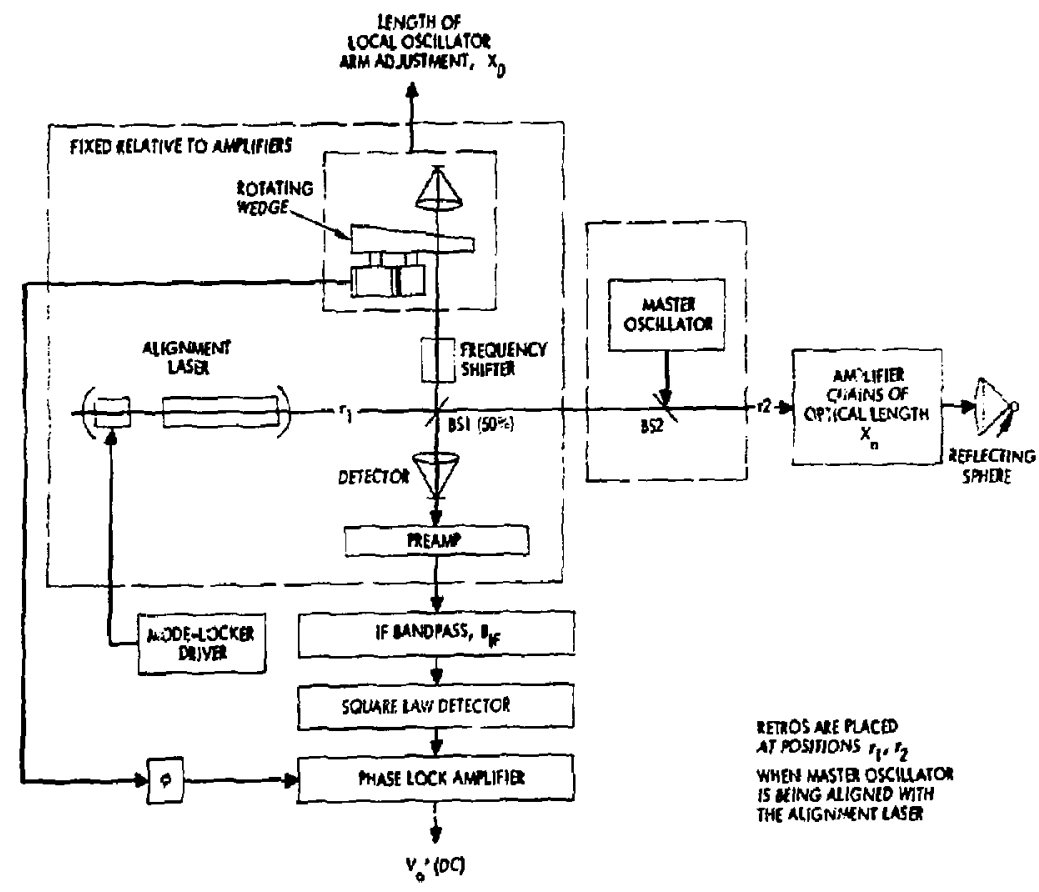

Figure 7-1. Proposed Configuration

equalization involves measurement of the precise location of the peaks of $v_{0}$ as a function of $X_{n}$ (which is separately adjustable). An effective way to measure the peak is to essentially differentiate it by inserting the rotating wedge and causing $X_{0}$ to vary as $X_{0}$ to vary as $X_{0}+\Delta X$ coswt, with $\Delta X$ small compared to $\mathrm{C}_{T}$. The signal $\mathrm{V}_{0}$ ar the rotation frequency w now goes through a null which can be found precisely if the pulse train is stable and the system SNR is high.

\section{ALIGNMENT}

The proposed system is easy to align. First, the alignment laser/receiver compiex is permanently positioned relative to the amplifier chain, with the exception of the LO retro/rotating wedge assembly. The latter assembly, however, causes no alignment problem, because the retro need never be positioned 

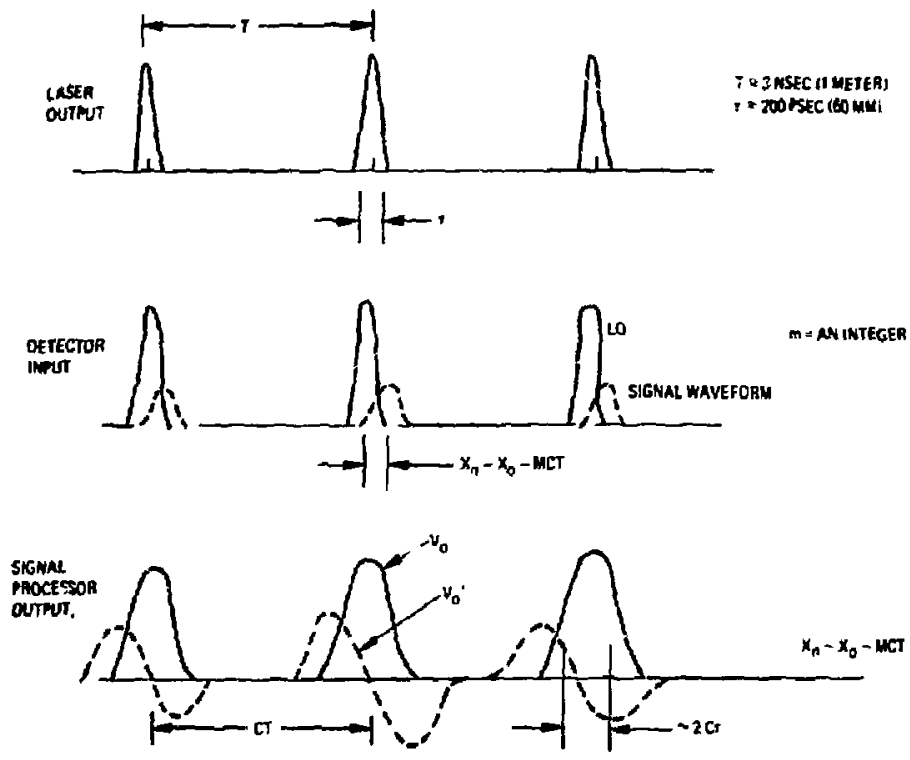

Figure 7-2. Principle of operation

precisely. Second, the $L O$ beam will always be automatically aligned with the signal beam at the detector because of the use of this same (LO) retro.

The alignment sequence wili proceed as follows:

1. The alignment laser is oriented properly relative to the amplifier chain by means of the lateral and focusing adjustments described in other sections of this report. Everything else is now aligned relative to this laser.

2. A retro is placed at $r 2$ and the beamsplitter (BSI) adjusted to maximize the signal $v_{0}$. The path lengthe $X_{n}$ are now adjusted in sequence for a null in $v_{0}^{\prime}$. 


\section{MATHEMATICAL RELATIONSHIPS}

The mode locked laser output may be written

$$
\begin{aligned}
E(t) & =\sum_{n} E_{n} \cos \left(\omega_{n} t+\alpha_{n}\right) \\
& =\operatorname{Re}\left[\exp \left(j \omega_{0} t\right) \quad \sum_{n} E_{n} \exp \left(j n \Delta \omega t+j a_{n}\right)\right]
\end{aligned}
$$

where the field $E(t)$ is first represented as a sum over longitudinal modes of frequency $\omega_{n}=\omega_{0}+n \Delta \omega(\Delta \omega=2 \pi / C / 2 L)$ and $\phi_{n}$ is the phase of each mode. For a properly stabilized mode locked laser, the phases $\phi_{n}$ may fluctuate a little around zero, without effecting significantly the amplitude of the mode locked pulse train at $t=0$ where the modes add in phase. The second form for $E(t)$ exposes more clearly the periodic nature of the pulse train; the sum is a phaser that varies slowly compared to the optical freguency $\omega_{0}$ (the period is $T=2 \pi / \Delta \omega$ ). As an example of the shape of the pulse train, let $E_{n}=$ constant $=1$ and $\phi_{n}=O$; then $(N=$ number of modes)

$$
E(t)=N \cos \omega_{0} t\left[\frac{\sin (N \Delta \omega t / 2)}{N \sin (\Delta \omega t / 2)}\right] \text {. }
$$

The function in brackets typically occur 8 in the theory of diffraction gratings and has secondary maxima which are small as $\mathrm{N}$ grows large $\langle\mathrm{N} \geq 20$ for a Nd:YAG mode locked laser). The width of the principal maxima peaks is approximately $\frac{2 \pi}{\Delta \omega^{\circ}} \cdot \frac{1}{N}$, and the secondary maxima are lower than the principal maxima by the factor $\sim \frac{1}{N \pi}$.

The detector response to a signal field

$$
E_{S}=y \sum_{n} E_{n} \cos \left(\omega_{n} t+a_{n}^{\prime}\right)
$$


and a local oscillator field

$$
E_{L O}=\sum_{n} E_{n} \cos \left[\left(\omega_{n}+\omega_{L O}\right)(t+t)+\phi_{n}\right]
$$

is proportional to

$$
R(\tau)-\left|E_{B}+E_{L O}\right|^{2}
$$

where $y$ is the attenuation of the signal path $\left(: \approx 10^{-9}\right)$ relative to the LO path. ${ }^{\omega_{1 O}}$ is the LO frequency shift, $T$ is the difference in propagation time, and $a_{n}^{\prime}$ is the distortion of the pulse train created by dispersion in the amplifier chain (signal arm). If the IF bandpass allows only a nar row range of frequencies near $\omega_{L O} \ll \Delta \omega$, then the IF output contains only the following signal term from $R(\tau)$

$$
\begin{aligned}
& S(\tau)-Y \sum_{n} E_{n}^{2} \cos \left[\omega_{L O} i+\left(\omega_{n}+\omega_{L O}\right) \tau+\Delta \alpha_{n}\right] \\
& =\gamma \operatorname{Re}\left[\operatorname{expj}\left(\omega_{L} L O^{t+\omega_{0}} O^{T+\omega_{L}} L O^{T}\right) \Sigma_{n} E_{n}^{2}\right. \text { expj } \\
& \left.\left(n \Delta \omega t+\Delta \alpha_{n}\right)\right] \text {. }
\end{aligned}
$$

The second form for $S(\tau)$ contains a sum factor that is very similar to that derived earlier for $E(t)$; in fact for $E_{n}=1$ and $\Delta \alpha_{n}=0$, it is the same. In addition, for $a_{n}=0$, the sum factor with $T=t$ is proportional to the power $P(t)$ in the pulse train. Thus, in calculating the SNR at $r=O$ (where SNR is a maximum), use the peak laser power for the LO conversion power instead of the average power which is appropriate for calculating the shot noise. For typical peak-to-average powers of 20 , the heterodyne SNR is therefore a factor of 20 better at $T=O$ than is obtained for the usual CW LO oscillator. 


\section{ESTIMATED ACCURACY}

The SNR ratio is now calculated assuming the beamsplitters BS1 and BS2 are both 50 percent

$$
\begin{aligned}
& P_{I} \text { (average) }=0.5 \mathrm{~W} \text { (la ser power) } \\
& P_{S}=\left(20 P_{L}\right)\left(\frac{1}{2}\right)^{4}\left(10^{-9}\right)=6 \times 10^{-10} \mathrm{~W} \text { (signal power) } \\
& P_{n}=\frac{2 h v}{\eta} \sqrt{{ }^{B} I F^{B}}=6 \times 10^{-15} \mathrm{~W} \text { (noise equivalent power) } \\
& \text { S:VR }=10^{5},
\end{aligned}
$$

where, conservatively taken,

$$
\mathrm{B}_{\mathrm{IF}}=10^{6} \mathrm{~Hz} \text { (IF bandpass) }
$$

$B=10 \mathrm{~Hz}$ (integration band - width of phase lock amplifier).

The width of the IF bandpass must be enough to pass any doppler shiśts present in the system. The two most important sources of doppler shift are

1. Adjustment doppler shift =

$$
\frac{2 v}{\lambda} \leq 2 \frac{(1 \mathrm{~cm} / \mathrm{sec})}{\left(10^{-4} \mathrm{~cm}\right)}=20 \mathrm{KHz}
$$

2. Dither doppler shift

$$
\begin{aligned}
& \pm \pm \mathrm{fd} / \lambda= \pm 100 \mathrm{KHz} \\
& \text { (for } \mathrm{f}=10 \mathrm{~Hz}, \mathrm{~d}= \pm 1 \mathrm{~mm} \text { ). }
\end{aligned}
$$

The accuracy available with a given SNR is readily obtained if the curvature near the peak is modeled by a gaussian. Let the shape of the signal as a function of $X=c T$ be given by

$$
v_{0}(X)=\exp \left(-x^{2} / 2 \sigma^{2}\right),
$$


where $3 \sigma$ is roughly the half power width of the curve which we take to be $6 \mathrm{~cm}$. The dither signal $v_{0}^{\prime}(X)$ is given by

$$
V_{0}^{\prime}(X)=\Delta X \frac{d V_{0}(X)}{d x}=v_{0}(X) \cdot \frac{x \Delta X}{\sigma^{2}}
$$

For equal no:se powers

$$
\frac{V_{0}^{\prime}(X)}{V_{0}(X)}=\frac{S N R}{S N R}=\frac{X \Delta X}{\sigma^{2}}
$$

The question can now be asked: For fixed $\Delta X$, how small can $x$ be hefore the dither SNR (SNR') is smaller than say $10^{\circ}$

From the last equation,

$$
\begin{aligned}
X & \leq \sigma\left(\frac{\sigma}{\Delta X}\right)\left(\frac{S N R}{S N R}\right) \\
& =(2 \mathrm{~cm})\left(\frac{2 \mathrm{~cm}}{.4 \mathrm{~cm}}\right)\left(\frac{10}{10^{4}}\right) \\
& =.1 \mathrm{~mm},
\end{aligned}
$$

where

$$
\begin{aligned}
\Delta \mathrm{X} & =4 \mathrm{~mm} \\
\mathrm{SNR} & =10^{4} .
\end{aligned}
$$

\section{ALIGNMENT LASER REQUIREMENTS}

An average power of $0.5 \mathrm{~W}$ was shown to be more than adequate for the path lengtli equalization measurements. It has been increased over the $0.1 \mathrm{~W}$ propised earlier to make easier and improve the accuracy of the designs for the lateral alignment sensors. In addition, a high degree of stability in average power is desired for the alignment task, so that a stabilized power 
supply is included. The essential features of the alignment laser are summarized in Table 7-1.

Table 7-1. Alignment laser parameters

\begin{tabular}{|ll|}
\hline Model & GTE Sylva lia 607 \\
Output Power & $0.5 \mathrm{~W}$ mode locked \\
& $1.6 \mathrm{~W} \mathrm{TEM}_{\mathrm{oo}} \mathrm{CW}$ \\
& $6 \mathrm{~W}$ multimode \\
& 200 psec \\
Pulse Wid̈th & 0.01 percent \\
Amplitude Stajility & Open loop; maintains mode lock several \\
Operation & hours without adjustment. \\
\hline
\end{tabular}

\section{POTENTIAL PROBLEMS}

There are two unresolved issues that might cause probicms:

1. Reflections from optical surfaces near the input to the amplifier chain are not highly attenuated and could mask the relatively weak signal.

2. Large flluctuations in the phases $\alpha_{n}$ of the modes making up the pulse train could lead to fluctuations in the position of the nuli joint and hence degrade the position measurement.

There are several factors that should reduce the presence of ghost signals. First, all optical surfaces are tilted except for the beam apodizers, and these introduce curvature in the reflected beam. The pinholes will greatly attenuate any ngtica: beams with the wrong curvature; whether this attenuation reduces the ghost reflections below the signal strength has not been calculated.

The effects of phase fluctuations are difficult to handle analytically because the behavior of the node locker must be known. However, since the mode locker driver is an open loop resonator adjusted in frequency to conform to the laser cavily mode separation, il would appear that any flucluations would occur on a pulse to pulse basis and be completely averaged out over the time constants $(-0.5 \mathrm{sec})$ characteristic of the final measurement 
bandwidth. In order to interfere with a sequence of 0.5 sec measuiements, these would have to be flucluations associated with time constants of durations from about 0.1 seconds to 0.1 hours. The re do not appear to be any such time constants in the alignment laser. Note that distortion in the pulse train due to dispersion is of no consequexice to first order becruse the pisase diffe:ence $\Delta \phi_{n}$ is proportional to $n$ (let $\Delta \phi_{n}=a n$ ) and the periodicity of the signal $S(t)$ is simply changed from $T=2 \pi / \Delta w$ to $I^{\prime}=(2 \pi-a) / \Delta w$.

It is felt that the se factors, although not quantitatively resolved, wi!! not materially degrade the projected performance. The attenuation of $10^{-9}$ assumed for the two way transmission through the ampliner chain uces not take into account aberrations which could lead to greatly increased attenuation at the pinhole. This factor could reduce the position accuracies estimated earlier, or would require more sophisticated signal processing. 


\section{IWO AXIS MIRROR GIMBAL}

\section{FUUCTIONAL DEFINITION}

The first mechanization to be discussed in this section is a device whose purpose is to perform the function of properly aligning the main laser beams on the target chamber and target pellet after they have exited the amplifier chain. The two specific alignment functions which must be accomplished are 1) centering the footprint of the beam on the target chamber focusing lens and 2) pointing of the beam to properly inter cept the target pellet. These two adjustments can be accomplished by imposing two-axis angular adjustment on the beam at two locations in it path as it travels from the last element of the amplifier chain to the target chamber. Since each chain has at least two turning mirrors aiong this path (two chains have three turning mirrors) it is an obvious choice to use these mirrors as adjustment elements operating on the beams. It is the only choice if the restric tion is imposed that no optical elements be added along the path solely for adjustment purposes. Thus it is proposed, and will be described in detail herein, that two turning mirrors in each laser chain be mechanized to provide two-axis angular adjustment to the laser beam path.

Before proceeding to a description of the various alternatives studied to accomplish two-axis adjustment of the turning mirrors, two topics will be reviewed as background information pertinent to the design considerations and tradeoffs concerning these adjustments. These topics are 1) the performance requir ements of the adjustment functions to satisfy overall system requirements, and 2) the environmental conditions as they exist at the location where these adjustments are to be accomplished.

\section{PER EORMANCE REQUIREMENTS}

The first requirement to be considered is what the operating mode of the adjustment device is to be; toanual, remotely actuated, or automatic. Due to the relatively inaccessible physical location of the turning mirrors and a system requirement that these adjustments be accomplished in a very short 
time period immediately preceeding an actual shot, it becomes a requirement that these adjustments be either remotely actuated (e.g. at the control console) or done automatically (e.g. servo controlled). To satisly the system accuracy requirements for pointing at the target the adjustment device must be capable of an angular resolution at least $0.4 \mu \mathrm{rad}$. This, and all the following requirements, apply to each of the two axes of adjustment. The accuracy requirement for centering the beam on che focusing lens calls for a resolution of at least $20 \mu \mathrm{rad}$. An active range of \pm 9 mrads is requized for remote or automatic angular adjustment of the turning mirrors. This requirement is dictated by the combined adjustment ranges for pointing and centering. The mounting orientation of the alignment device must be capable of being adjusted over a range of $\pm 40 \mathrm{mrads}$ and with a resolution of at least $80 \mu \mathrm{rads}$ upon installation. As mentioned earlier, the adjustment device must be capable of both remote and automatic operation. The automatic mode, in which adjustment would be servo-controlled, imposes furcher requirements on the physical characteristics of the device. First, the surning mirror must have a natural resonant frequency of greater than $10 \mathrm{~Hz}$ and must have a damping factor of greater than 0.1. These are necessary in order to implement a stable servo loop of reasonable bandwidth. There are no specific requirements on the device concerning linearity or hysterisis, as long as the other requirements are met.

\section{ENVIRONMENTAL CONDITIONS}

The turning mirrors jor all of the laser chains are mounter onto the massive space frame which surrounds the target chamber. When these mirrors are used for two-axis centering and pointing they must satisfy the performance requizemusts reviewed above while being exposed to the environmental conditions which exist at the space frame location. The ambient air temperature is $24^{\circ} \mathrm{C}$ nominally and is controlled to vary a maximum of $\pm 0.5^{\circ} \mathrm{C}$. This variation will be cyclic in nature with a period of approximately 15 minutes. The air is flowing vertically from the ceiling to the floor with a velocity of 0.4 meters $/ \mathrm{sec}$. The maximum ceiling to floor temperature differential is $1.0^{\circ} \mathrm{C}$. Dita on the estimated vibration spectrum 
shows resonant peaks at approximately $16 \mathrm{~Hz}$ and $23 \mathrm{~Hz}$. The amplitudes of vibration at these peaks correspond to acceleration levels of $5.6 \times 10^{-6} \mathrm{G}$ : $\mathrm{s}$ at $16 \mathrm{~Hz}$ and $4.5 \times 10^{-6} \mathrm{G} \cdot \mathrm{s}$ at $23 \mathrm{~Hz}$.

\section{GIMBAL TECHNIQUES}

To begin the study of a device capable of meeting the pointing and center ing adjustment requirements a review was made of the various techniques available for suspending the turning mirrors such that their angles of inclinaticn could be adjusted. The various gimbal configurations considered fall into two categories: 1) those which have a center of totation at the mirror surface, and 2) those whose center of iotation is behind the mirror surface.

\section{Quad Pod}

The quad-pod is a gimbal technique which has a center of rotation at the mirror surface. This type of configuration is illustrated in Figure 8-1. With this particular technique the mirror and its mounting cell comprise the middle or coupler link of a four-bar linkage. The legs upon which the mirror stacds connect the mirror to ground. The linkage pivots to ground at the

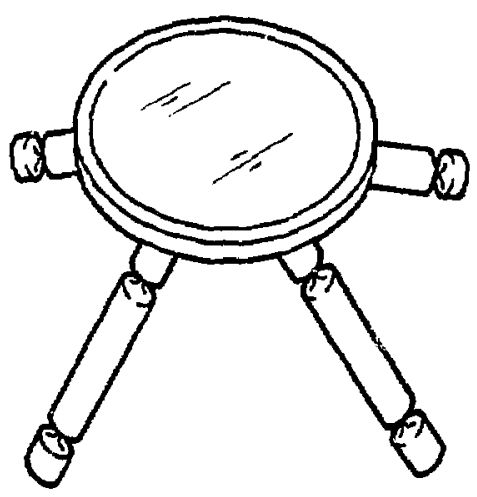

Figure 8-1, Quad pod gimbal technique 
base of each of the legs and pivots at the interface between each leg and the mirror. An instantaseous center of rotation at the mirror surface is achieved by designing the gimbal such that lines extended trom each of the supporting legs will intersect at the surface plane of the mirror. When this is the case, it is seen that as each leg rotates through small angles about its base pivot the motion at the mirror end of the legs will be tangent to the inter section point just defined. Thus, if this point is designed to be at the miror surface and two other points on the mirror structure (i.e. the leg pivot points) are caused to move tangentially to a circle whose origin is at this point, the mirror will be rotating about its own surface, as illustrated in Figure 8-2. This argument holds true only for snall angle movements, such as those required for the adjustment range of the mirrors. For small angles, the pivot points can be advantageously configured as flexurc pivots rather than using pinned joints or bearings. The use of flexure pivots offers the advantages of eliminating friction and backlash, imposing only springrate. For the very small angle resolution required, friction and backlash should be avoided to as large a degree as is possible. This gimbal technique is adaptable to becoming a two-axis type device simply by standing the mirror on four legs rather than two. This is illustrated in Figure 8-1. It is from this configuration that the name quad-pod is derived. The configuration requires that each of the flexure pivot points be capable of pivoting in two directions. This can be readily accommodated by having each of the four iegs be symmetrically necked down adjacent to the mounting points at the base and at the mirror.

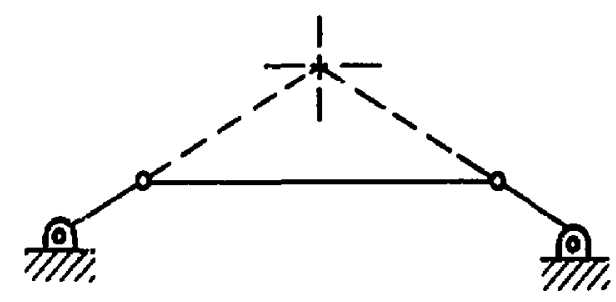

Figure 8-2. Center of rotation for quad pod gimbal 


\section{Edge Flexures}

The mirror can also be rotated about its own surface by mounting it betxeen two pivot points whose axis lies in the ceflecting plane of the mirror. This type of configuration is illustrated in Figure 8-3. The mirror and its cell ar: basically comprising an axle between the two pivot points. Bearings or pir red joints could be used as the pivots, but again, as was the case with the quad-pod described earlier, it is preferrable to use flexure joints to eliminate friction and backlash. A type of flexure which could be used for this gimbal cenfiguration is the cross-strap, illustrated in Figure 8.4. The flexure is made up of two sheet metal straps oriented at right angles to each other, one end of each strap being secured to the base structure and the other end of each to the gimbaled element. This type of flexure allows rotation in the desired plane by means of cantilevered beam flexing of the two straps. The flexure is very stiff in resisting rotations in any axis other than the desired one and is also very stiff in resisting translations. This is due to the fact that any of these undesired motions can occur only when the straps are ilexed about an axis having very high moment of inertia or are loaded in pure tension or compression.

In order to adapt this second technique to a iwo-axis gimbal configuration, a gimbal ring must be employed, as is illustrated in Figure $6-5$.
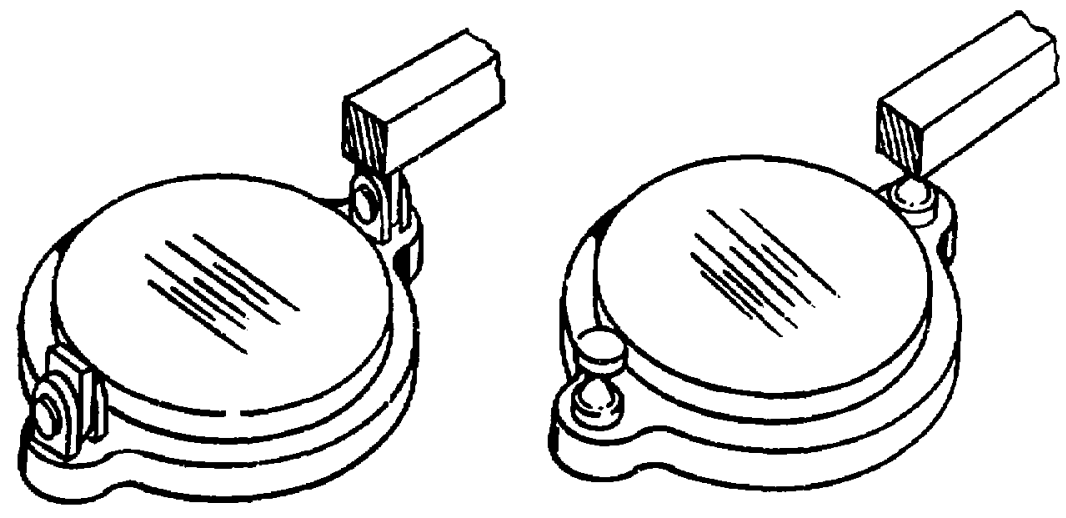

Figure 8-3, Double pivot gimbal 


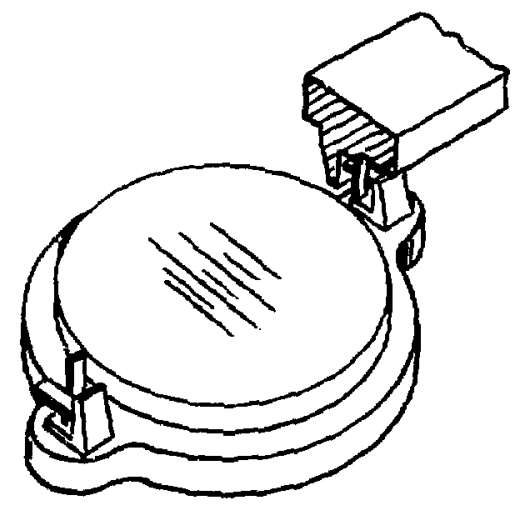

Figure 8-4. Cross-strap flexure pivot gimbal.

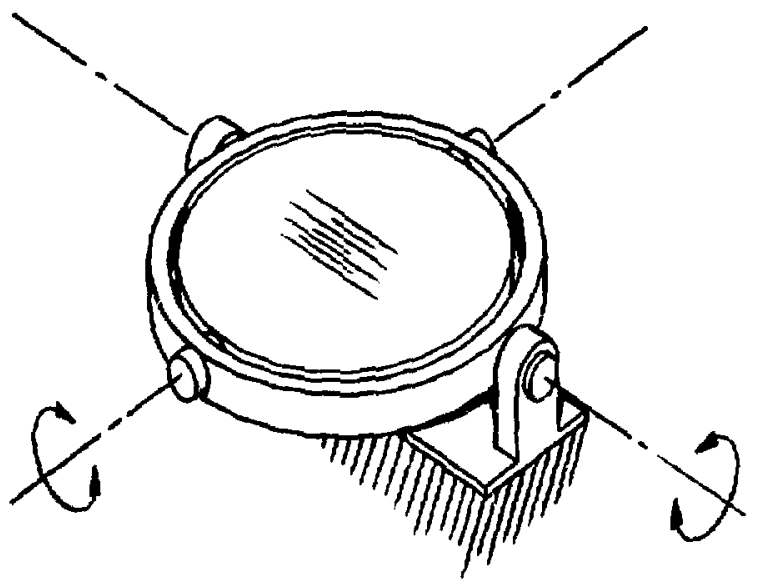

Figure 8-5. Two axis gimbal using gimbal ring

\section{I wo-Axis Flexure Column}

If the center of rotation for the mirror is allowed to be inehind the reflecting surface, this introduces other possibilities for mirror suspension. A gimbal technique which could be used for this application is a two-axis 
flexure column. This type of configuration is illustrated in Figure $8-6$. Hughes as considerable experience in using this type of gimbal system on steering mirrors for various laser pointing systems. The operating concept is very simple. The mirror can be made to assume various angle orientations by causing the flexure to bend as a cantilever beam from its base. For the small range of centering and pointing angles required, such a technique can be applied, The flexure column will exhibit very negligible friction and backlash. It also appears that for small angles, having the center of rotation behind the mirror surface will not adversely effect the way the mirror functions in the pointing system.

\section{Universal}

Other options for gimbaling the mirror behind its surface are to use either bearings or cross-strap flexures arranged in a universal type pivot. There are commercially available components which utilize the cross strap flexure technique discussed carlier. An example of these is shown in the accompanying data sheet, Figure 8-7. A universal type pivot is basically similar to concepts using a gimbal ring which were discussed earlier. But by moving the center of rotation to belind the mirror the size of the gimbal ring and the spread between suspension points can be greatly decrezsed, making a more compact gimbal device.

\section{Comparisons}

Before proceeding to a discussion of various means of actuating the mirror, a brief discussion of some of the relative advantages and disadvantages of the various gimbal techniques studied will be useful. There are

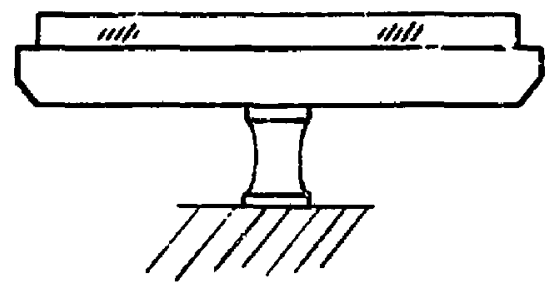

Figure 8-6. Two axis flexure column 


\section{Flexural Universal Joint}

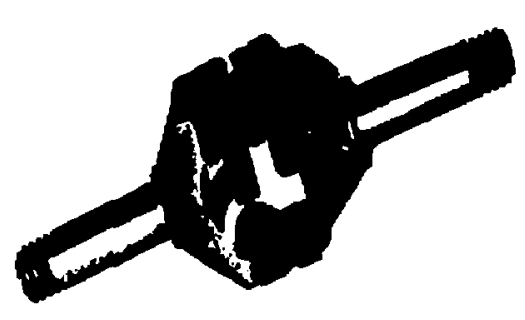

Sizes

1/2" Jiameter to 3" alometer over center of jolnt using standerd internal parts.

Larger dianotorg are availoble with spactat perts.

Cannecting shufes to suit applirmtion.

\section{Features}

Wa vear

Friectaniess

No abbing ports

Smooth running

we backlash

Ho lubrication

One-piece asseably ready for use

flexiblffty of count ing conflguration

Easy installiction

Elibinates expenslue repalr and overhaul
Handix fluld Dower bivision has on exeleing new approoch to unlversel joints for crecision Iastrulents and controls. slexibilley of the jolnt it atcoined through the use $0^{x}$ peraanently installed

flexure aeubers. Ir conbining proven opoduction atehods frae the flexural pivot pro. duet line, it is now possible to supply ready asseabled. frictionless,universal joints desigred for high=volune epplications.

\section{Materials}

\author{
STAMQARD - AISI 420 and \\ AI5: 410 brazed \\ construction.
}

SPECIAL - Designed for welded consiruction apallication.

FOR SDECISIC REQUIREMENTS, COUTACT:
The Bendla corporation
fluid Power Division
211 Seward Avenue
Ueice, Mew rork ijsoj
Flex-pivot soles
(315) 797-2500

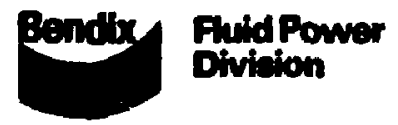

Figure 8-7. Bendix data sheet 
several areas in which we can conceptually evaluate and compare the various types of devices without taking each to a detail desiga level; these are: 1) mechanical comp exity and number of parts required, 2) desirability of the location of the required gimbal parts, 3) adaptability of the concept to a twoaxis gimbal device, 4) location of center of rotation, 5) size of device/space sequired, 6) friction and backlash, and 7) relative cost. The relative strong points of the quad-pod type gimbal technique are that all of the gimbal structure is behind the plane of the mirror while the center of rotation is still at the reflective surface, that the concept is readily adaptable to a two-axis configuration, and that if flexure type pivots are used the gimbal exhibits negligible friction and becklash. Some possible drawbacks to the quad + pod technique are that it is relatively complex, and therefore relatively expensive. It is complex in the sense that a two-axis gimbal would require eight two-axis flexure joints. This can be compared to a two-axis flexure column gimbal which, while it does not have its center of rotation at the mirror suriace, requires only one two-axis flexure joint. The space required for the quad-pod device can be tailored to meet design constraints. There are an infinite number of choices for the length and angle orientation of the legs of the de ce thus making the size of the gimbal a design variable.

The gimbal technique which employs pivots in the plane of the nirror on both siojes offers the advantage of not having the gimbal structure extending backwards behind the plane of the mirror for any great distance. The relative importance of this depends on the mounting concept and space limitations on the space frame. Adapting this type device for a two-axis gimbal requires a gimbal $r$ ing. While the use of a gimbal $r$ ing is not technically complex, it does add extra parts and also makes it more difficult to achicve high mechanical resonance. A two-axis device using a gimbal ring would require four pivots, Again flexures would be desirable.

The two-axis flexure column, while it does have a center of rotation behind the mirror surface; does have several strong points. The entire gimbal reouires only one part, even for two-axis freedom. This part does extend rearward behind the mirror cell, but requires very litcle space. Friction and backlash are negligible, and low cost is inherent in the lack of complexity and low number of parts required. 


\section{ACTUATING IECHNIQUES}

Various concepts and methods for actuating the gimbal mounted pointing and steering mirrors will now be discussed. The primary requirement which any actuating technique must satisfy is moving the mirror to its required location with adequate resolution and maintaining that location after all power is removed from the actuating and sensing devices. Achieving a resolution of $0.4 \mu \mathrm{rads}$ requires very small mechanical motions. If, for example, the actuation is applied at a radius of $25 \mathrm{~cm}$ from the center of rotation, motions of $0.1 \times 10^{-6}$ meters must be resolved and held. Thus, in considering actuating co.icepts, it is obyious that stention must be paid to eliminating ant mechanical backlash and isolating components of mechanical tolerance.

Reduction Linkage

The first concept to be discussed is a linkage reduction device, illustrated in F tgure 8-8. The device is shown in the figure for only one axis of actuation and is moving 2 mirror gimbaled with two pivots. A single axis actuator wili be described first, and then its adaptability to a two-axis configuration. In this concept, the mirror cell becomes the output member of the linkage which is shown schematically in Figure 8-9. An angular motion into the inpu: link drives the mirror through a reduced angular motion. As is shown, the input link is extended to achieve a high mechanical advantage. The engle reduction ratio of the linkage is $\theta$ out $/ \theta$ in $=b / a$. The arc-length of the input at the end of the extender arm is $X$ in $=\theta$ in (c). The change in mirror angle for linear motion input is then $\theta$ out $=X$ in $\left(\frac{b}{a \varepsilon}\right)$. By making $b$ snall and $a$ and $c$ large, very small angle changes can be achieved with reasonable si\%e mechanical motions. Figure $8-10$ illustrates how this concept might be implemented mechanically to accomplish the required mirror adjustments. The translation input to the linkage reduction device is provided by a stepper motor driving a ball screw. The linkage is configured using a saw cut block to provide flexure pivots. By using flexures pivots and a preloaded ball

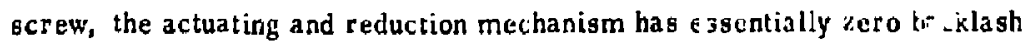
and very low friction. The mochanism shown has a predicted oerformance of $0.5 \mu \mathrm{rad}$ mirror angle change for an input of 4.8 degrees to the ball screw shait. The mirror is gimbaled with its center of rotation at the reflecting 


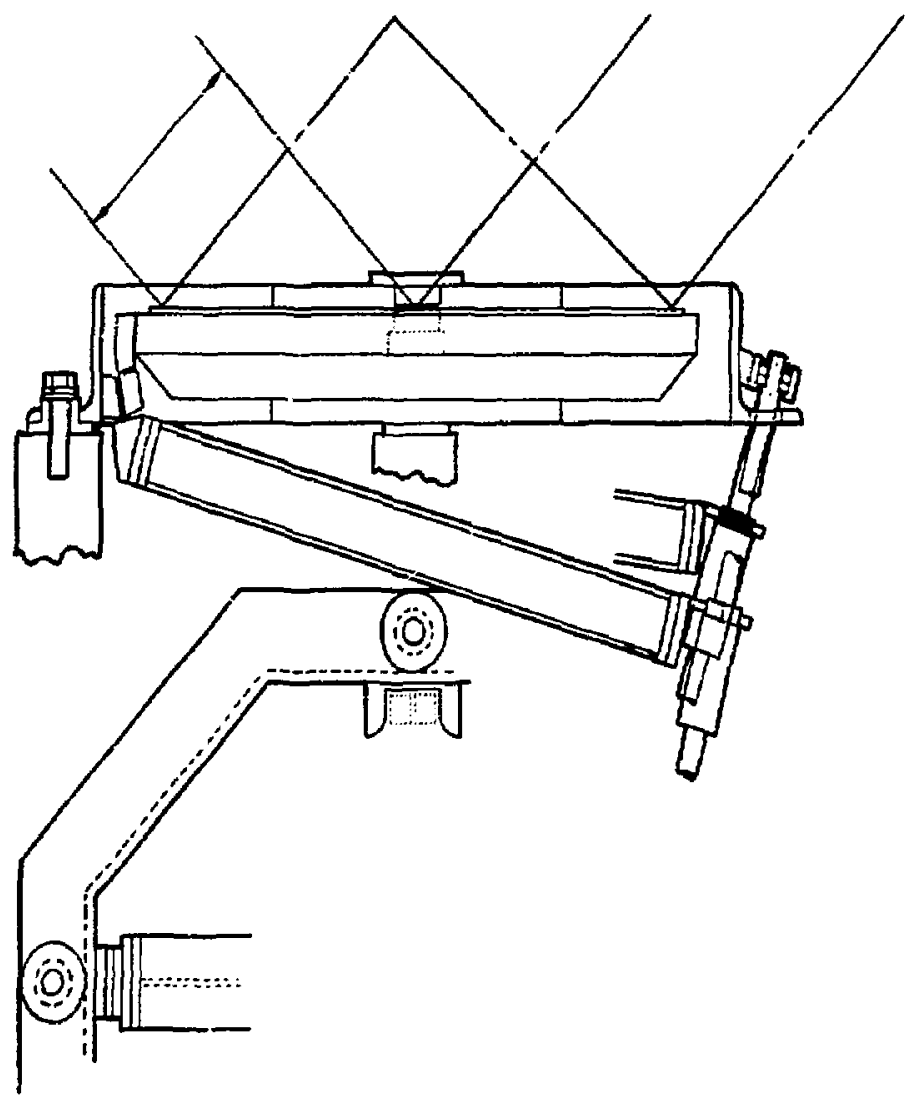

Figure B-8. Linkage reduction device (steering mirror layoul)

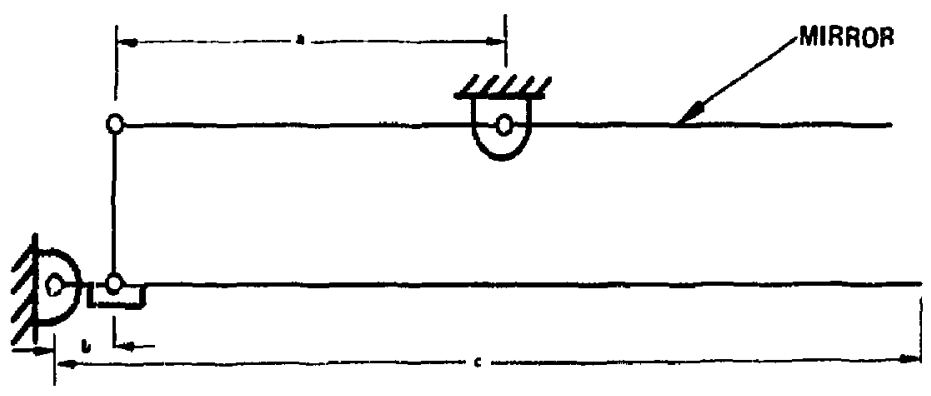

Figure $8-4$. Linkage reduction device schematic diagram 


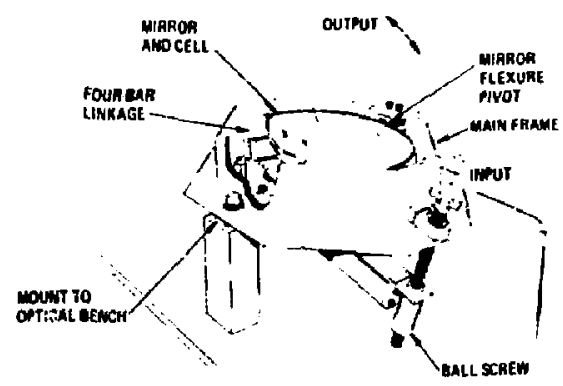

Figure 8-10. Linkage reduction device

sut face using flexures on either side. This is a single-axis type gimbal and thercfore a gimbal ring must be used to adapt for two-axis operation. Also, the actuation/reduction mechanism for the inner gimbal ni:st be mounted to and move with the gimbal ring, while that for the outer gimbal would be mounted to the base structure. The linkage reduction technique appears to have several disadvantages. It is relatively complex (and therefore costly) due to the fact it requires at least four flexures for a single axis and possibly as many as six. it is also bulky and would require considerable space behind the mi:ror, especially if tro mechanisms were used. Inch Worm

Also studied was an inch-worm actuating concept capable of actuating/ indexing in increments small enough so that no intermediate reduction mecharism would be required. The concept is illustrated in Figure 6-11. The device consists of two electro-mechanical clutches and a piezo-electric crystal, which compose the carriage, and a shaft, composing the stationary base. The two shaft-type clutches, one of which is normally open and the other normally closed, are secured to either end of the hollow crystal. The carriage may then be stepped in inch-worm fashion along the shait by sequencing through the following steps: 1) excite the crystal, causing it to lengthen a very small increment, 2) exile the normally open clutch, catusing it to grip the shait, 3) excite the normally closed clutch, causing it to release the 


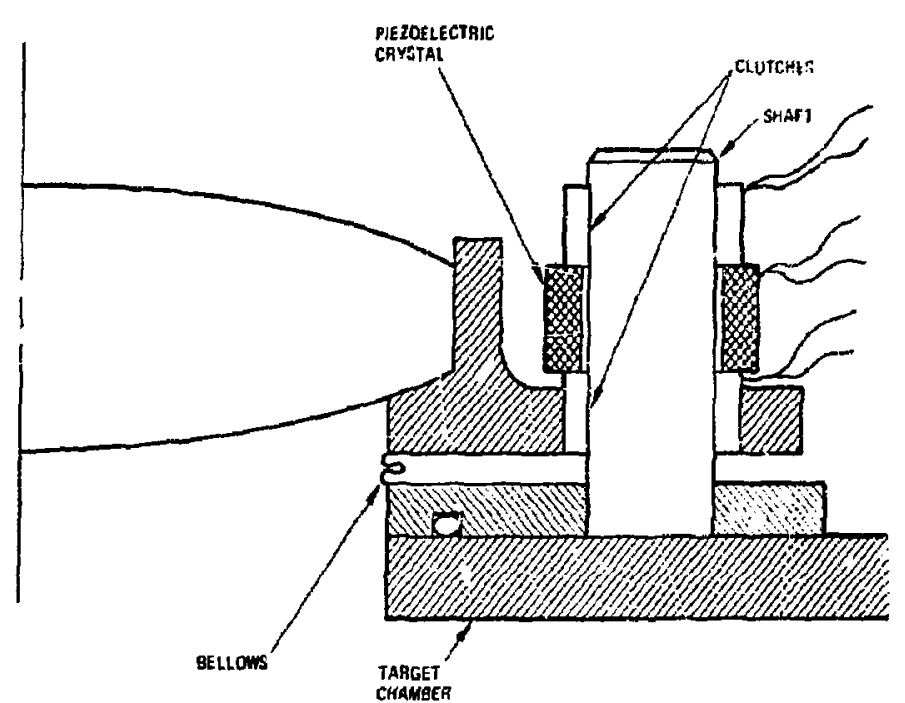

Figure 8-11. Inch-worm device:

shaft, 4) remove excitation from the crystal, causing it to shrink to its friginal length. 5) remove excitation from the normally closed clutch, calising it to regrip the shaft, and (6) remove excitation from the normally open shaft, causing it to release the shaft. Either the carriage or the shaft couid be mounted to the periphery of a gimbaled mirrar with the opposite half teing mounted to the base. The step size can be tailored by design of the nomina! length of the piezo-electric crystal and the roltage applied to it. Advantages to this actuating concept are that it is mechanically simple and it occupies very little space. Possible disadvantages are that the electronics reçuired weuld be relatively complex (compared to simply driving a motor), the device is not easily adaptable to twn-axis gimbal techniques since decentering would cause problems, and the technique could be relatively expensive to implement.

\section{Spring Ratio}

A concept utilizing the ratio of two springs was alos studied as a reduc:tion technique. Figure $8-12$ is a schematic representation of how the concept functions. The mirror is mounted on a gimbal system which exhibits a very high angular string-rate $\left(\mathrm{K}_{1}\right)$, such as a two-axis flexure column. Mounted to the mirror periphery are two linear eprings of values $\mathrm{K}_{2}$ and $\mathrm{K}_{3}$ at 


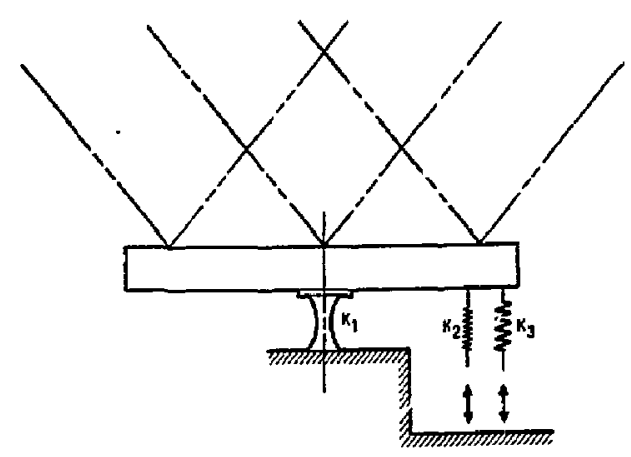

Eigure 8-12. Spring ratio reduction technique

distances $r_{2}$ and $r_{3}$ from the center of rotation. The effective angular spring rates of the two springs are $\mathrm{K}_{2}\left(r_{2}\right)^{2}$ and $\mathrm{K}_{3}\left(r_{3}\right)^{2}$, respectively. The parameters $K_{2}, K_{3}, r_{2}$, and $r_{3}$ are chosen so that $K_{3}\left(r_{3}\right)^{2}$ is approximately equal to $K_{1}$ and $K_{2}\left(r_{2}\right)^{2}$ is much much less than $K_{1}$. Translation inputs into the unattached end of $\mathrm{K}_{3}$ are used to accomplish coarse adjustment of the mirror angle and input into $K_{2}$ are used for fire adjustment. Expressions for change in mirror angle $(\theta)$ for small translation inputs $(\Delta)$ into either $K_{2}$ or $K_{3}$ can se derived with little difficulty. Balancing torques on the mirror we have for an input of $\Delta$ into the coarse spring $K_{3}$;

$$
\Sigma i=0=K_{1} \theta+k_{2} \theta\left[r_{2}\right]^{2}-K_{3} r_{3}\left[\Delta-\theta r_{3}\right]
$$

Solving for the mirror angle change pex translation input we have;

$$
\theta_{c} / \Delta=\left[K_{3} r_{3}\right] /\left[K_{1}+K_{2} r_{2}^{2}+K_{3} r_{3}^{2}\right] . \text { (Coarse) }
$$

Similar expressions can also be written for inputs to the fine adjustment spring, $\mathrm{K}_{2}$ ' 


$$
\begin{gathered}
\Sigma I=0=K_{1} \theta+K_{3} \theta\left[r_{3}\right]^{2}-K_{2} r_{2}\left[\Delta-\theta_{2} r_{2}\right] \\
\theta_{F} / \Delta=\left[K_{2} I_{2}\right] /\left[K_{1}+K_{2} r_{2}^{2}+K_{3} r_{3}^{2}\right] \quad \text { (Fine) }
\end{gathered}
$$

This particular reduction technique offers several advastages. It is very simple mechanically, having only a small number of non-complex and relativeiy inexpensive parts. The concept is readily adaptable to two-axis operation, since the linear springs are capable of complying wish smail angle changes due to adjustments in tuo-axes, Only a small space is reguired to implement this concept. Friction and backlash are essentiaily eliminated. And a straightforward method of actuating the free end of the springs can be used, such as a stepper motor driving a ball screw.

\section{BASELINE DESIGN}

Based on all the various concepts studied for mirror gimbaling, actuation, and motion reduction, a baseline was chosen upon which is develop a detailed design of a mechanism to accomplish centering the laser beam on the focusing lens and pointing at the target pellet through precision angular adjustment of the turning mirrors. This baseline design which will be described in detail beiow, is the design which is proposed to be the best overall methor of accomplishing the required adjustments.

\section{Configuration}

The type of gimbal structure chosen for the baseline design was the twoaxis flexure column. This type of gimbal has many advantages, with the only possible disadvantage being that tise center of rotation is lccated behind the reflective surface. For the very small range of angular adjustment required this was judged not to be a serious problem. This technique provides a twoaxis gimbal using besically only one part other than the mirror cell and the base structure. It is therefore very compact, free of complexity and inexpersive. The gimbal is inherently a two-axis device, thus eliminating any arlaption to accomplish two-axis operation. As was stated earlier, Hughes has considerable experience building hardware which applies this gimbal 
technique. There are several shapes which can be used for the llexure column, namely cylindrical, spherical, and elliptical. Analysis and hardware experience have both shown that the best results are obtained with a flexure column having an elliptical profile. This particular shape offers the best trade-off of characteristics for stress per unit angle, elimination of stress concentrations, and rigidity against undesired motions. An example of the type of analysis which has been done to evaluate the characteristics of the elliptical profile flexure is given in Appendix G.

Chosen to actuate the mirror was a combination of the spring type reduction technique being driven by a stepper motor/ball screw assembly providing translation input. This particular combination offers many advantages. It is non-complex, both mechanically and electrically, and relatively inexpensive. The technique is easily adaptable to two-axis operation, requiring no itcrease in complexity over a single-axis device. It also has the characteristics of very low friction and backlesh. The spring reduction concept is very advantageous because it serves to greatly reduce any errors attributed to slight errors or tolerances in the stepper motor, shaft, or ball nut. And it does this without having any mecharical tolerances of its own. In a system where resolution on the order of one millionth of an inch is striving to be achieved this becomes very important. Mechanical tolerances simply cannot be held that close, as evidenced by the fact that Bureau of Standards gage blocks are accurate to only about 5 millionths of an inch. The adjustment of the mirrors occurs due to a very very slight change in the force balunce on the mirror rather than attempting to input a very small translation. The transiation input is, rather, into a very weak spring, and thus can be very large compared to millionths of an inch. All of the components required for the reduction/actuation mechanism (springs, st/pper motors, ball screws) can be of commercial type quality and still achieve the ultra-precise adjustments required. This will be shown later, in the description of a model device which was built and tested.

Now will follow a description of the actual physical design which has been developed to accomplish the lunctional requir ements for centering and pointing. This is a product design of the baseline concepts and techniques which have just been diecussed. For the product design, the coarse and the fine 
adjustment springs have been drranged differently than was shown in the schematic diagram of the concept in Figure 8-11. Rather, the springs have been arranged coaxially, with the fine spring and its actuator assembly riding on a carriage which is the actuator end of the coarse adjustment spring. This is illustrated in Figure 8-13. Reference to this figh.e will make it easier to understand the detailed mechanical design of the mechanism. It should also be noted that the rotary input from the stepper motor goes into the ball mut, rather than the shaft.

The detailed design of the actuation/reduction mechanism is sbown in Figure 8-14. Following through an adjustment sequence will serve to describe the decailed parts and their operation. To begin, it is assumed that the pointing (or centering) sensor has detected an error in the laser bean alignment which must be corrected. A command is given to the coarse stepper motor to step, and it does. The motor drives the coarse preloaded ball screw through a rotation of one step size of the motor. The motor is coupled to the ball screw through a bellows coupling (to prevent side loads

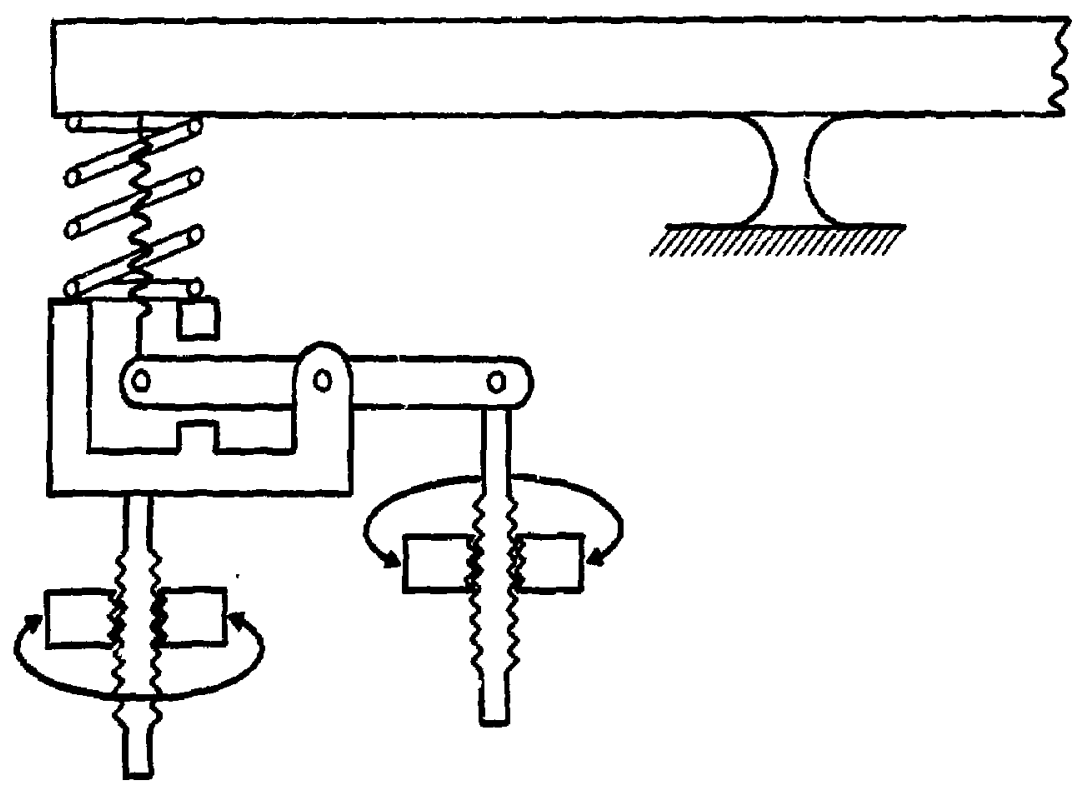

Figure 8-13. Spring ratio actuator device 


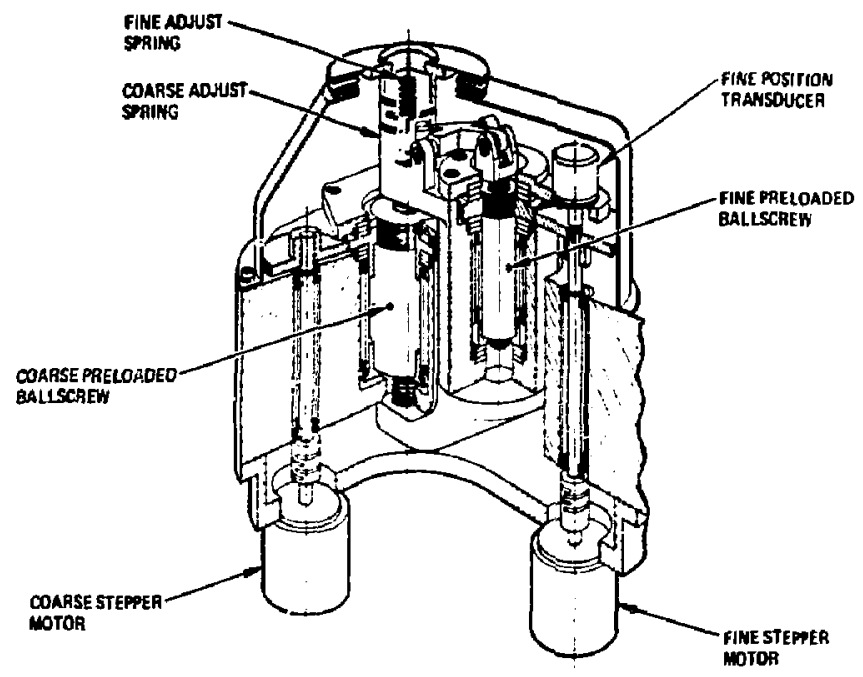

Figure 8-14. Mechanical design - spring ratio actuator device

on the motor shaft) and through a gear pair. The gears are used to reduce the total extended height of the derice. As the ballscrew is rotated, the shaft translates a distance equal to its lead multiplied by the ratio of one step size to $360^{\circ}$. The length of the coarse spring is in turn changed slightly, varying the force into the mirror cell. The mirror cell, being mounted on its gimbal flexure, changes angle according to the expression developed earlier. Commands continue to be given to the coarse motor until the mirror alignment comes to within a certain threshold around null which is within the range of the fine adjustment spring. During coarse adjustment, the fine adjustment mechanism has been carried along on its carriage, as can be seen by referring to the figures. Once the fine adjustment threshold has been reached, commands cease to be given to the coarse motor and start to be given to the fine stepper motor. When this motor is given a command it steps, causing the fine preloaded ball screw to rotate, being couplat to it through a bellows coupling and a pair of mated gears, similar to the coarse. Iranslation of the fine adjust shaft causes a change in length of the fine spring, the shaft being coupled to the spring by means of a rocker arm asstmbly. The coarse spring remains stationary during the fine adjustment sequence. The change in length of the fine springs causes a very small change in force into the mirror cell. This small force is working against two very stiff 
springs, the coarse adjust spring and the gimbal flexure. Thus, an ultra-small change in mirror angle results. The button at the very top of the mechanism in Figure $8-14$ is the point which exerts force on the mirror cell. The mirror and its cell are not shown in this figure. Figure 8-15 show's what the complet. installed two-axis device might look like with the mirror and its ceil, the gimbal flexure, a coarse/fine actuation/reduction mechanism for each axis and the base struclure.

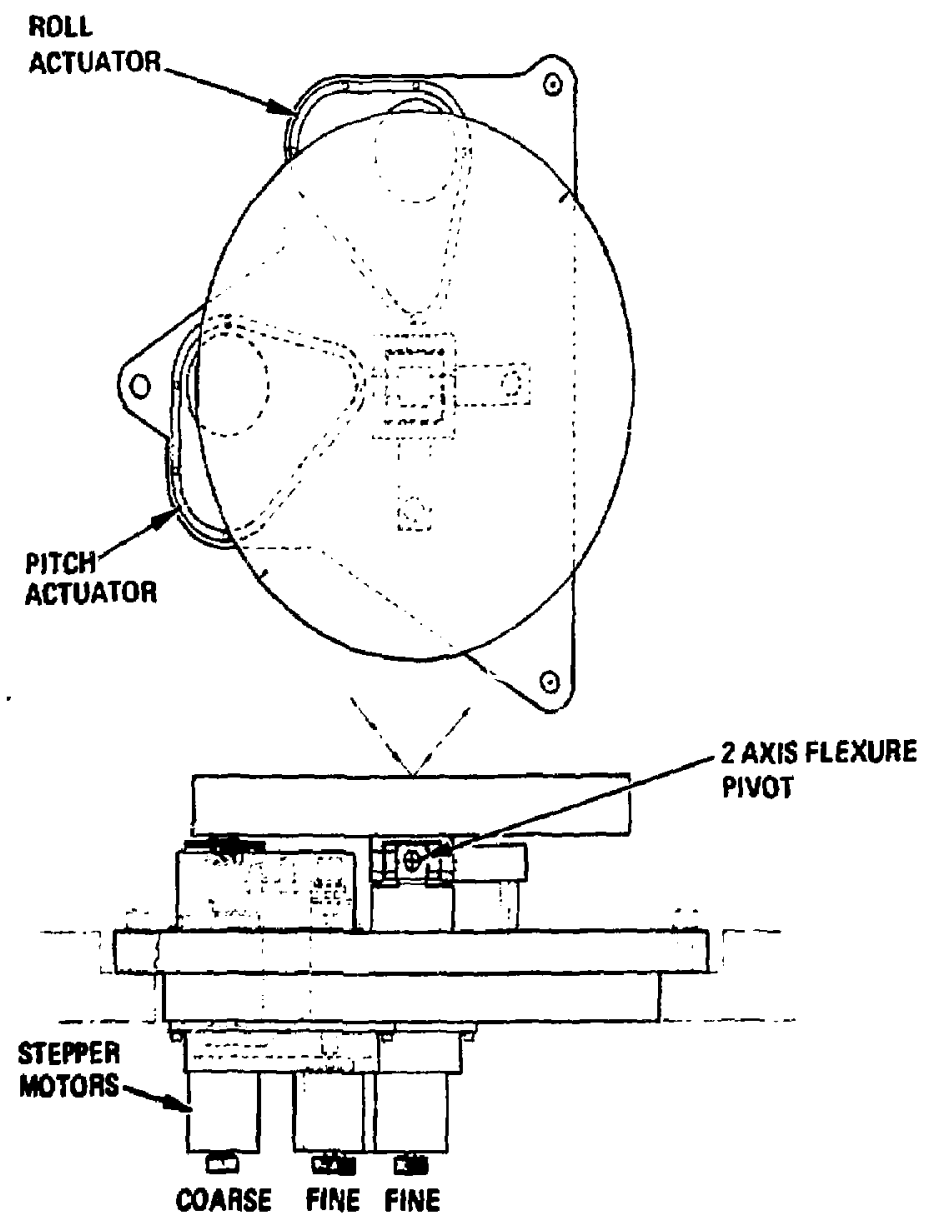

Figure B-15. Two-Axis mirror actuating assembly 


\section{Performance Parameters}

The mechanical description leads next into a discussion of the physical and performance parameters of the device. The two-axis flexure column gimbal was designed to have a spring rate of $1.4 \times 10^{6} \mathrm{in}-\mathrm{lb} / \mathrm{rad}$. The coarse and fine adjustment springs were chosen to have values of $1.4 \times 10^{4} \mathrm{lb} / \mathrm{in}$ and $140 \mathrm{lb} /$ in respectively. Each acts a radius of 10 inches from the gimbal center. All of the stepper motors have a step size of $1.8^{\circ}$ and the ball screws all have a lead of 0.050 inches. Combining these parameters yields a scale factor of $25 \mu \mathrm{rads}$ per coarse motor step and $0.2 \mu \mathrm{rads}$ per fine motor slep. Data sheets on the various comnercially available parts which are components in the mechanism have been included in Appendix $C$.

Polential problems in the actual operation of this device are errors due to thermal dissipation and undamped resorances. The thermal dissipation problem is handled by insuring that the power which is dissipated as heat in the stepper motors is not able to initiate transients in the reduction mechanism and support structure. Although it does not show explicitly in Figure 8.14, this is accomplished by placing insulating shims between the motors mounting bases and the associated mounting structure, and by exposing the motors to the flowing air, maximizing convection. Using these techniques, it is possible so make the thermal resistance for heat conducting into the base structure several orders of magnitude greater than that for convection to the air. This will insure an isothermal structure, avoiding errors in alignment due to temperature changes.

Controlling resonances will probably take a semi-empirical approach during the fabrication and testing of the first mechanisms. It is difficult to predict at what frequency the primary resonance will occur, except that every effort should be made to have as high a ir equency as can be obtaired. Techniques which can be appiied 10 add damping and control resonance are the insertion of damping materials between the mirror cell and the base structure, and also the use of metals having high damoing characteristics for varinus parts such as the mirror cell, the gimbal flexure, and others. One such material is Incramute, a copper alloy. A data bulletin on this material has 
been included in Appendix K, An elastomeric damping material inserted between the mirzor cell and tire base was used successfully to control resonance on the model device which has been built and tested. This will be described in greater depth further on in this repori.

The product design for the two-axis mirror adjustment mechanism has been accomplished with the following in mind. The design is such that boith the pointing and centering functions can be accomplished by the same derict. This is eificient not only from a design and development standpoint, but also in terms of parts procurement, spares, and maintenance. Also the design can easily accommodate upgrades from manual to emote to automatic. The actuation/reduction design used is compatible with all of these modes.

TEST MODEL

Design

A breadboard type rest model was designed and built, using Hughes funds, to verify that the techniques proposed here for ultra-precision mirror pointing are feasible. Undertaking this task was possible because of the relatively simple mechanical approach and the commercial availability of the principle components. The concepts and technigues of the test model device are identical to the proposed product design, with some modifications to the physical arrangement to make the device easy to test and relatively quick and easy to fabricate. The test model design is illustrated in Figure $8-16$. The test modci was constructed to be only a single-axis anjustment device. It is felt that the feasibility of the design can be adeguately verified by testing only a single axis. Referring to Figure 8-16, the test model had two stepper motors and two ball screws. These drove the coarse compression coil spring and fine extension coil spring. These springs were attached to a bracket on the rear of the mirror cell. The mirror cell was gimbaled to the base by a flexure column. For the test model the gimbal hexure had a spring rate of $14,000 \mathrm{in-lb} / \mathrm{rad}$, the coarse and fine springs had rates of $120 \mathrm{lb} / \mathrm{in}$ and $1.5 \mathrm{lb} / \mathrm{in}$ respectively. They were mounted at radiuses of 10 inches and 8 inches from the gimbal center. Thus the angular spring rates for these are $12,000 \mathrm{in}-\mathrm{lb} / \mathrm{rad}$, and $96 \mathrm{in}-\mathrm{lb} / \mathrm{rad}$. Both stepper motors had a step size 


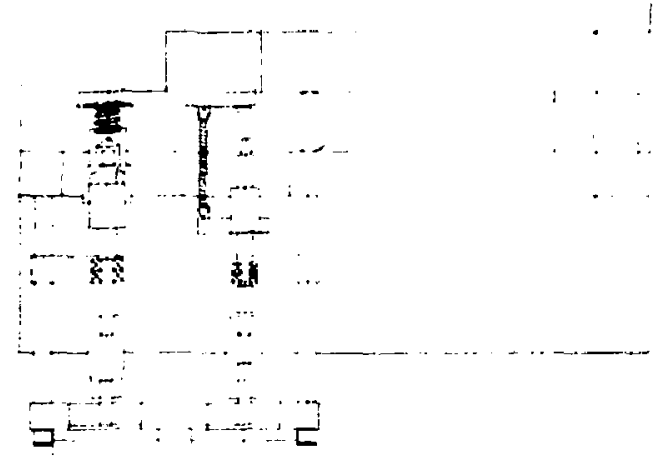

Figure 8-16. Mechanical design - test model device

of $1.8^{\circ}$. The coarse ball screw had a lead of 0.1 inches while the lead on the fine ball screw was 0.05 inches. These parameters lead to calculated scale factors of 23 irads per step for the coarse adjustment and $0.12 \mu \mathrm{rads}$ per step for fine. The inertia of the mirror was also determined and its resozance was calculated to be $10 \mathrm{~Hz}$.

Testing

The test device was set-up on a granile slab type optical bench. An zutocolimator was arranged to measure the angle of the mirror using a test configuration which is shown in Figure 8-17. A detailed series of tests is

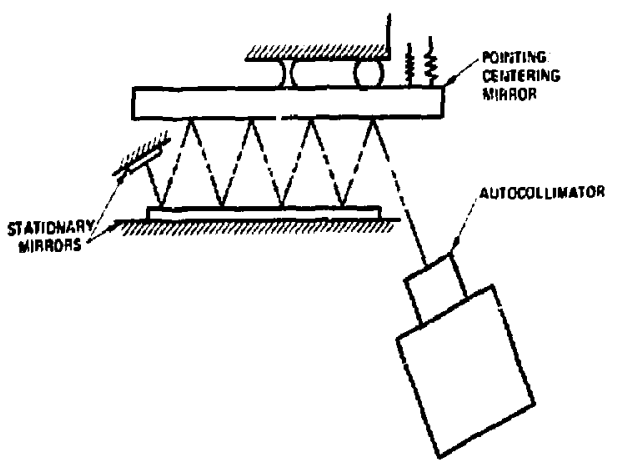

Figure 8-17. Test Configuration 
in the process of being run and the results will be available soon. Quick-lock preliminary tests have been accomplished, yielding the following results. The coarse step size was measured to be nominally $29 \mu \mathrm{rads}$. The fine step size was measured to be nominally $0.15 \mathrm{\mu rad}$. The total variunce in step size in a sample of 50 steps was approximately \pm 15 percent. The bysterises in going up $\mathbf{5 0}$ steps ard then returning was approximately 3 percent. During preliminary tests it was observed that each time a coarse step was taken the mirror was excited to resonate at approximately $8 \mathrm{~Hz}$ and it appeared to take about 30 seconds for the vibration to die away. The mirror did not appear to be excited when a fine step was taken. Throu, home experimentation it was discovered that by wedging a sphere of high-damping elastomer between the mirror cell and the base no evidence of the lightly damped tesonance could be observed. These ojservations bear out what could be intuitively reasoned, that the spring inertia system is by itself very lightly damped and will resonate freely, and that the vibration car be controlled by inserticn of damping material between the mirror inertia and the base structure. The actual performance of the device agreed well with the calculated predicted performance. This, in conjunction with the fact that the design technique used for the test model is very similar to the proposed design, adds greater conidence to being able to successfully design, fabricate, and operate the proposed design. 


\section{FRONT-END POSITIONLNG}

\section{FLNCTIONAL DESCRIFTION}

The next group of adjustment functions for which solutions were studied are those which must be accomplished at the beginsing of the laser uptica! chains before the beams enter the amplifier nodules. There are a large number of alignnent functions which fall into this category and these will tie briefly described. The master oscillator, the source of the 20 lase: beams, must be precisely alizned to the master pinhole. This encompasses the following specific alignment parazreters: If two-axis ( $x$ and $y$ translation adjustment and 2/ iwo-axis (a and $\beta$ ) angular adjustment. Adjusting these four parameter allows the master oscillator beam to be properly ceatered on the finhole and to exit the pinhole at the proper angle. The sane alignnent funct:on requige ents which exist for the master oscillator are al so necessary for proper adjustment of the alignment laser. Its beam too must be adjusted in $x$ and $y_{0}$ and $a$ and $\beta$ to properly orient it to the pirhole. After these wo adjustnents are acconplished the master oscillator and the aligmnent laser are then boresighted to each other. Xext, the oscillator bench, which mounts the master osciilato:, alignnent laser, and master pinhole, must be aligned to the bench nounting all of the bearn splitters for the 20 chains. This adjustment consists of translation is $x$ and $y$ and of rotation in $\alpha$ and $\beta$. The exisiance of this adjustment capability makes it possible to realign trom the oscillator bench to all 20 laser channels (after they are initiall $\%$ aligned with a single adiustment, rather than 20. This will be very valuab.e when pertubations occur on tile osciliator bench. Once the master oscillator beam has beun split into 20 beas.s, each beam mest then have the following adjustrients. fiach must be centered on its apodized aperture and each must also be cemered on the final spatial filter at the end of each amplifier chain. Both of these functions can be acconplished by imposing $x$ and $y$ translational adjust.ment and a and fotational adjustrinent on each bean at some point before it enters the apodized aperature. And iastiy, all of the 20 laser bans must be adjusted so that the total path length of each is equal. This may be calied a translation adjustnsent in the direction. 


\section{PERFORMANCE REQLIRENENTS}

The quatitative and quantitative requirements on all of the adjustment functions intcoduced will now be considered. First. for the alignment of the master oscillator and the alignment laser to the pinhcle, the beam must be adicstable in $x$ and $y$ over a range of $1.4 \mathrm{~mm}$ and with a resolution of $3.6 \mathrm{gm}$. In angle, the beam must be adjusted in 0 and $\beta$ over a range of 0.1 mrad and with a resolition of $1.8 \mu \mathrm{rads}$. The adjustments to the alignment laser beam need only be done in a manual mode, but the adjustments to the master oscillator beam nust be accomplished automatically to satisfy total system requirements. To properly align the 20 beams to their apodized apertures and spatial i1lters. the capabitity of adjusting the beams with a resolution of 175 microns over a range of $10.5 \mathrm{~mm}$ in translation and with a resolution of $35 \mu \mathrm{rad}$ over a range of $7(10) \mu$ rads in angle must exist. These adjustments to the individual laser chains seed only be accomplished in a manual mode. The path length adjistment ior each bean must be accomplished with a resolution of $11.7 \mathrm{~mm}$ over a range of $\$ .5 \mathrm{~cm}$. This function zequires manual mode adjustment only.

\section{ADJUSTMEXT TECHNIQUES}

Various concepts and tecinniques which can be applied to accomplishing the $x, y, z, y$ and $\beta$ iranslation and angle adjustments described will now be considered. It shculd be noted that the adjustnents considered here are perforned on the beant at a point where its physical diameter is much snalier and its powe much lower than was the case for the adjustments at the far end of tise laser chain, considered earlier. Also the resolution requirements are less stringent. Thus it is pussible to consider simpler techniques and smaller mecianisms tha. for the previous adjustments. A possible solution for the $\alpha$ and $\beta$ angular adjustnents would be to mourt a smail mirror on a two axis flex :re column. For the functions requiring only manual adjustment, the mirror c suld be driven by some very simple means, such as micrometer heads. F'or a case where autonatic adjustment is required, the micrometer neads could le adapted to be riotor driven. Care would have to be taken that the torque characteristics of the motor and friction of the micrometer head 
were compatible with the resolution required. It appears that the sesuluticn and range requirements could be met using simple mechanical parts and commercially available parts.

A concept which appears to be a very simple, ..t effective trethod of accomplisining $x, y$, and $z$ translation adjustments is to relay the laser bear into a corner reflector which can ba translated in three-axes. A strong point of this concept is that maintaining precise angular alignment of the corner reflector is not critical since the in-going and out-going beams are always parallel. Thus, adjustments in translation will never have any effect on angular alignment. The properties of the corner reflector are such that as it is translated, the output beam moves with respect to the input bearn by twice the amount the reflector is moved. That is, if the reflector is moved $1 \mathrm{~mm}$ in the $\mathrm{x}$ dirention, the relative displacement between the in and out going beams will change by $2 \mathrm{~mm}$. In using this concept it is necessary to prevent the beam from ever stradling the fold lines of the corner reflector, thus maintaining its polarization state.

A concept using two plane mirrors mounted on a parallelogram linkage was considered for accornplishing path lengt! adjustment and is illustrated in Figure 9-1. Moving the linkage causas the path which the beam follows to

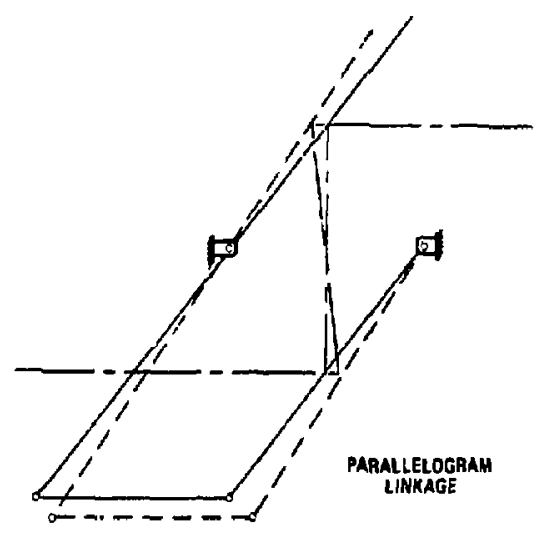

Figure 9-1, Path length adjustment concept 
change, without changing the exiting angle of the beam or the location of the centerline. Rigidly mounting the two mirrors together and rotating this rigid assembly would also accomplish path length adjustment but it would cause the exiting beam centerline to wander. Thus, the unique prcyerties of the parallelogran linkage are required to apply this concept. Figure 9-2 illustrates what this type of adjustment device might look like. Flexures are shown at the pivot points to eliminate friction, backlash, and misalignment. It is apparent that the complexity and cost of implementing such a device make it compare poorly with a very simple technique, such as a translating corner reflector. Thus it is not considered to be a serious candidate for proposed adjustment mechanisms.

Another option which was studied to accomplish adjustments at the front end of the laser cheins was to use commercially available optical adjustment stages, mounting the element necessary to accomplish the adjustment on the stage. For example, a two-axis tilt stage with a mirror mounted on its

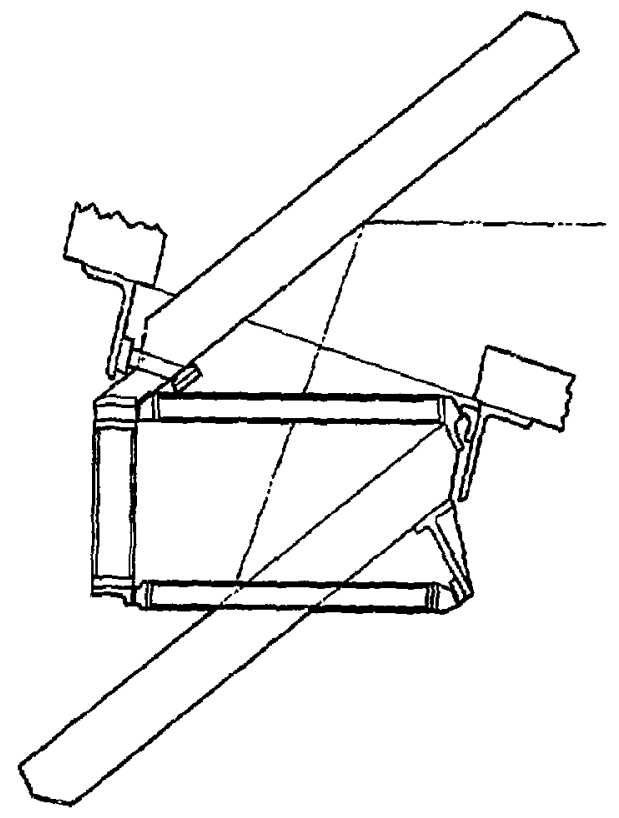

Figure 9-2. Mechanical design - path length adjustment device 
rotating platform could be used to accomplish $\alpha$ and $\beta$ angle adjustments. Likewise, a linear translation stage with a corner reflector mounted on it could be used to accomplish either $x, y$, or $z$ translation of the beam, depend on the orientation between the direction of stage movement and the beam path. The use of commercial stages offers several advantages. The devices have designed in provisions for the mounting of optical elements. Also, even though designing and building similar devices would not be difficult from a technical standpoint, it would be very difficult to be cost and schedule competitive against devices which are already designed and in production. If devices are available which can satisfy the resolution, range, and operating mode requirements discussed earlier, it appears advantageous to use them where possible.

During the discussion of the required adjustment functions at the front end of the laser chains it became obvious that there was redundancy in the types of functions required. All the various points which required adjustment required two axis translation and two axis rotation. The only added requirement is adjustment along the beam path in each of the twenty beams. it appears therefore that one adjustment mechanism device could be designed and enough of them built to use at every location where adjustment is required at the front end. This concept should be cost effective both from the standpoint of design and development expense and from the standpoint of parts procurement in quantities. Such a device, capable of performing all front end adjustment functions, will be called the front end positioner (FEP). The FEP will become part of the proposed system baseline and a discussion of its mechanization will now be presented.

\section{BASELINE DESIGN}

If a universal FEP is to be used, it must satisfy the most stringent requirements put upon it by all the various adjustment functions. Comparing all the requirements shows that the FEP must have a resolution capability of $3.6 \mu \mathrm{m}$ in translation over a range of $1.5 \mathrm{~cm}$. Likewise in rotation, the FEP must be capable of 1.8 prads resolution over a range of 7.2 degrees. The FEP must also be capable of being operated in either a manual or an automatic mode. 
Figure 9-3 shows the proposed FEP device. It is capable of adjusting the orientation of the outgoing beam with respect to the incoming beam in five parameters, $x, y, z, \alpha$, and $\beta$. It accomplishes this by having the beam reflect off of the three surfaces of a corner reflector and of of a turning mirror, nominally at 45 degrees. The corner reflector can be translated in three directions by the use of precision translation stages. Shown in the baseline design are Aerotech Inc, devices, model number ATS301MM. Mounted orthogonally, these translation stages provide the $x, y$, and $z$ adjustment functions, with the corner reflector mounted on the innermost stage. An Aerotech Inc. model number ATT185-5 two-axis tilt table is used to accomplish $\alpha$ and $\beta$ adjustments. Mounted on the stage is a turning mirror arranged to intersept the beam as it leaves the corner reflector. All of these devices are packaged onto a base structure, making a compact assembly which can be taken and mounted at all the various locations in the front end, as required.

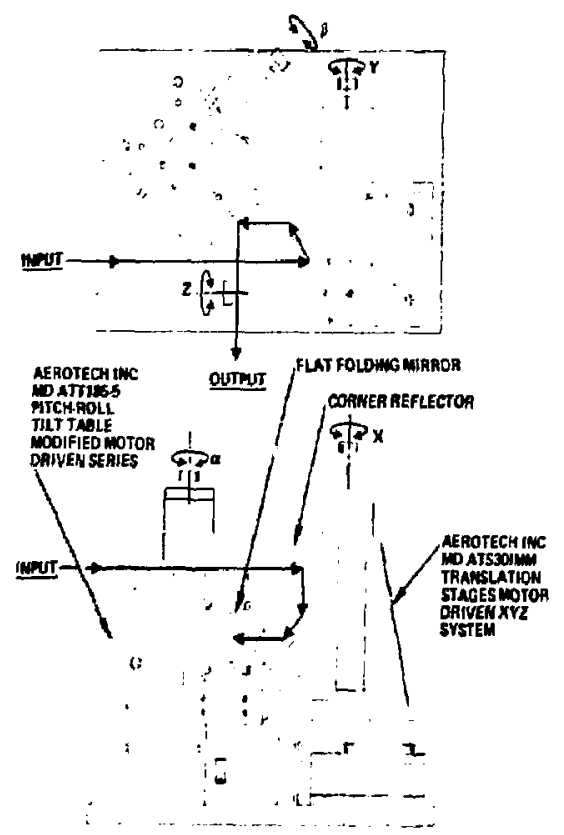

Figure 9-3. Front end positioner (FEP) device 
Included in Appendix $C$ are data sheets from Aerotech Inc, en the translation and tilt stages used in the FEP. Some of the more important parameters will be reviewed here. The linear translation stages can be procured either as manual or as motor driven devices. For the manual configuration the scale factor is $2.0 \times 10^{-6}$ inches per degree infut to the adjustment knob. The motor driven device used a 1.8 degree per step stepper motor and has a scale factor of $2.0 \times 10^{-6}$ meters per step. The range of either stage is 1.0 inch. The two-axis tilt stage is available from Aerotech only in a manual configuration. This device has a scale factor of 0.4 acuseconds per degree input to the adjustment knobs and has a total range of \pm 10 degrees. To satisfy the automatic operating mode requirement the device would hâve tn be modified to be motor driven after it wa s procured. Inquiries were made to Aerotech concerning the feasibility of this niudification. They concurred that it could be accomplished, taininis cart to provide flexible coupling between the motor shaft and the tilt stage dris'e shait and providing a motor with 35 oz-inches torque, minimum. Several other questions concerning scale factors, resolution definition in manual nodes, quantity discounts, retrofit, and temperature and creep sensitivity were discussed with Aerotech. Both the letter of inquiry and herotech's response are included in Appendix C.

This proposed design of the FF.P gives a compact relatively inexpensive device which is capable of universally accomplishing all adjustment functions at the front end. It meets all of the resolution and range requirements and meets the operating mode requirements with slight modification. And it appears to be very adaptable into the systems configuration of the entire front end of the laser gystem, 


\section{APERTIRE LNDEXING DEVICE}

\section{FIXCTION AYI REQLIRENENTS}

The eensing technique being used requires that two difierent si/es of aperture stops be indexed into the beam path upon command. The stops must be capabla of being remotely indexed into and out of the beam path with a very high degree of accuracy and repeatability. The two stop sizes required are $2.0 \mathrm{~mm}$ and $18.0 \mathrm{~mm}$ diameters. lipon being indexed into the bean, the stops must be concentric to each other and to the beam within 0.0003 inch, and their location must also be repeatable to within 0.0003 inch.

\section{DESIGiN}

Shown in Figure $10-I$ is the design concept which is proposed to satisfy the requirement stated above. The two stops are each mounted in an actuatur arm which is piroted to the base of the mechanism. The stops are aligned upon installation in the arms such that both centerlines are equidistant from the pivot point. Each actuating arm is driven by a rotary solenoid having a 90 degree step. The solenoids are arranged such that when in the unexcited state the stops are out of the beam path. If either solenoid is actuated, it swings the arm into the beam path. Very precise position indexing is accomplished by having hand tooling buttous on the arm and on the base structure come into contact. The solenoid holds this contact until power is removed, and thus power must be applied only when a stop is indexed into the beam. 


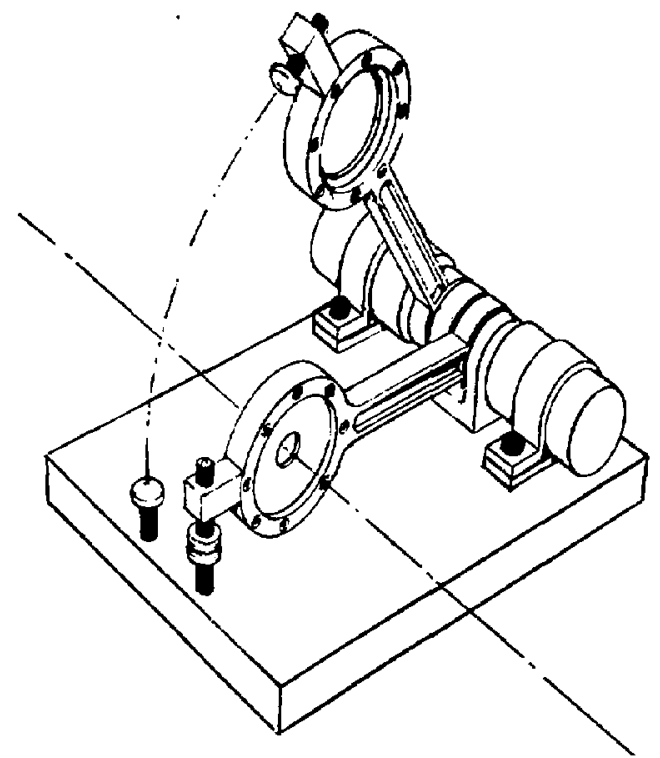

Figure 10-1. Aperture indexing device 


\section{OPTICAL DESIGN AND ANAL YSIS}

Those parts of the optical design and analysis efforts that involve quantitative ray trace evaluations are presented in this section. These evaluations are in support of the overall system design effort and fall into two general activity categories:

1. System synthesis and configuration evaluation.

\section{Sensor Design}

System synthesis and configuration evaluation was performed quring the early phases of the program in support of system concept generation. This effort was concerned with the geometrical setup of the alignnent system and how the alignment beam is generated and propagated to the various sensor packages. Several possible system designs were first considered from which a baseline optical configuration was selected. This was followed by a series of parametric tradeoffs based on ray tracing to determine the geometrical relationships between the size of surrogate targets and the alignment beam.

Sensor Design effort was concerned with the detailed design of the focus, pointing and final lens centering sensors. Detailed performance evaluations were made and positional sensitivities determined. Based on data generated in the parametric tradeoff study, three variations of the baseline configurations were analyzed. Systematic diffraction evaluations were carried out to determine the susceptibility of sensors to various input aberrations. Sensitivities were then studied in which performances of different sensors were analyzed after taking into account the presence of input beam pointing errors and position errors of the sensor package and the surrogate target.

These two activities are described in a general overview in the following sections. They are then described in detail in subsequent sections. 


\section{SYSTEM SYNTHESIS AND CONFIGURATION EVALUATION OVER VIEW}

\section{Initial Consideration of Possible Design Solutions}

The focused beam impinging on the surrogate target (a spherical ball) creates a virtual image $I_{0}$ inside the ball. This image in turn serves as the virtual object for the reflected alignment beam. There are basically five different ways (see Figure 11-1) in which the alignment beam can be reflected back toward the focusing lens, depending on the location of $I_{0}$ relative to the ball position. In Figure 11-1, Case 1 corresponds to the setup with $I_{0}$ at the center $f$ the ball. This is the condition for the input beam to be autoreflected back along its own path. The alignment beam zan therefore be sensed either at the region close to the preamplifier pinhole, or at a suitable location after passing through the focusing lens.

In Case 2, the position of $I_{0}$ is at a distance $d$ from the front surface of the ball, with d less than half the radius, $r$, of the ball. In this case, the reflected alignment beam is convergent.

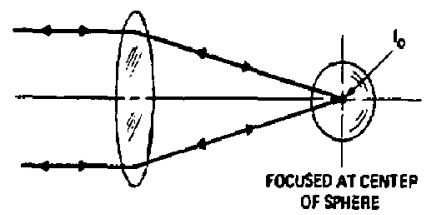

CASE 1

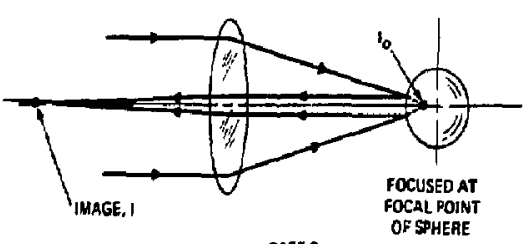

CASE 3

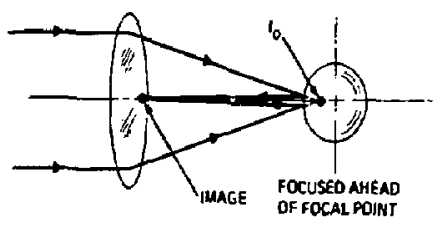

CASE 2

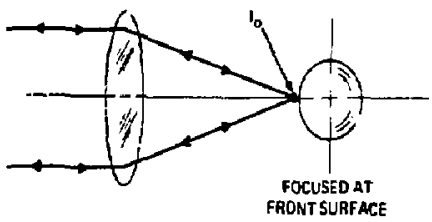

CASE 4

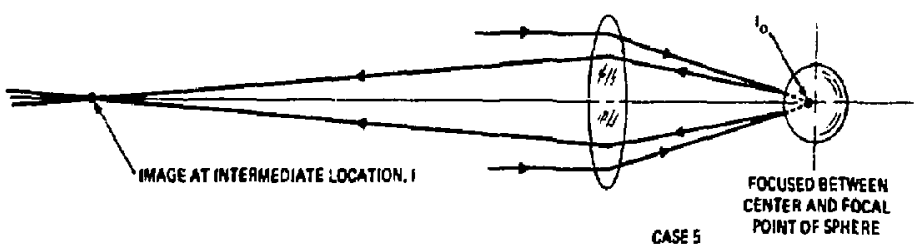

Figure 11-1. Five ways of reflecting the alignment beam 
in Case 3, $i_{0}$ is located with $d=r / 2$ (i.e. at the focal point of the spherel. The alignment beam is reflected off the ball as a parallel beam and, after passing through the fucusing lens, a real image is formed at the focus of this lens.

in Case $4, I_{0}$ is located at the front surface of the ball and the alignment beam is reflected back to the lens with the wavefront rotated through $180^{\circ}$.

In Case 5, $I_{0}$ is located with $r / 2<d<r$ and the final image, $I$, is formed beyond the front focus of the focusing lens.

With minor variations to the above cases, alignment schemes have been generated and sensors devised to detect the returning alignment beam. Figures 11-2 through 11-7 show six possible alignment configurations.

In Figure 11-7, the alignment beam has a small numeric al aperture aiter emejging from the central portion of the focusing lens. Because of the large difference between the numerical apertures of the focusing lens and the alignment beam, the sensor package has reasonably loose tolerances on its reference location relative to the target. The angular alignment of the sensor is carried out with the aid of a retro-reflector as shown schematically in Figure 11-7.

Of the six alignment configuraticns outlined above, configurations in Figures $11-4,11-5$ and $11-6$ were not considered attractive because of the need for mechanical motion. The configuration shown in Figure 11-3 was rejected because it required the use of large optics and, more importantly,
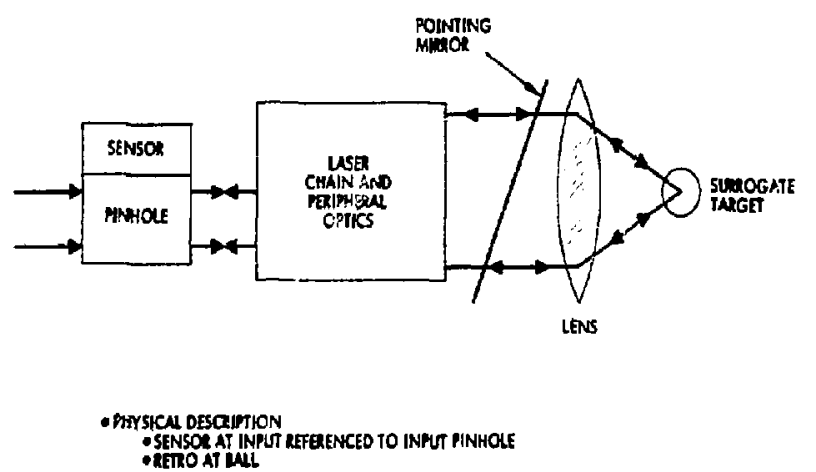

- SENSOA AT INPUT REFGENCEO TO INFUT PINHOL

Figure 11-2. Alignment configuration with sensor at input referenced to input 


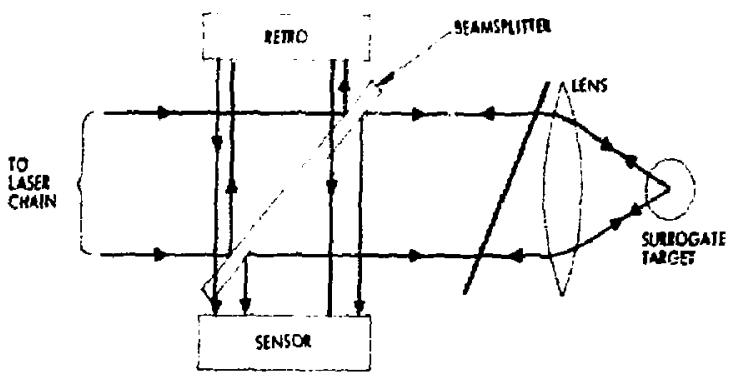

PAYSICAL OESCAIPTION

SENSOI NEA FCOUSING LENS

SENSOR REFERENCE TO INFJ OEAM VIA BEAMSPLITTER

AND RETRO

ANDO A aul

Elther fULL OR PARTIaL APERTIME

Figure 11-3. Alignment configuration with sensor nea $r$ focusing lens

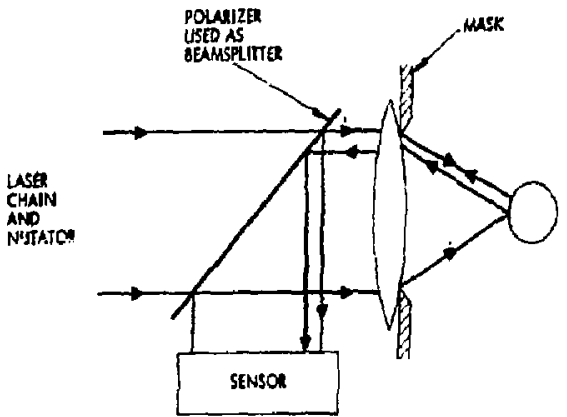

PHYSICAL DESGIPIION

IMAGE ON WASK AT EOEE DF FOCUS LENS

NUTATING WEO GE NLA PINHOLE FO ALL CMARINES

MONULATION OCCU AT NASX

SENSOR IS ENERGY-IN-WLCKET TYIE

Figure 11-4. Alignment configuraticn witi image on mask at edge of focus lens 


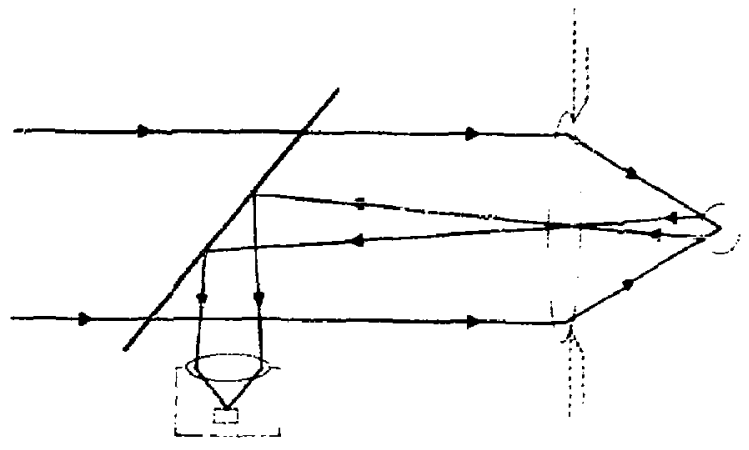

DhYSICAL DESCAIPTION

IBEGE IN PLANE OF FOCUSINE LENS

MASK AT OUIE EDGE IS REFELENCE LOA HOLE IN CENTER

Of LENS)

FCCUSINO LENS REIMAGEU ON DETECTOR

Figure 11-5. Alignment configuration with image in plane of focusing lens

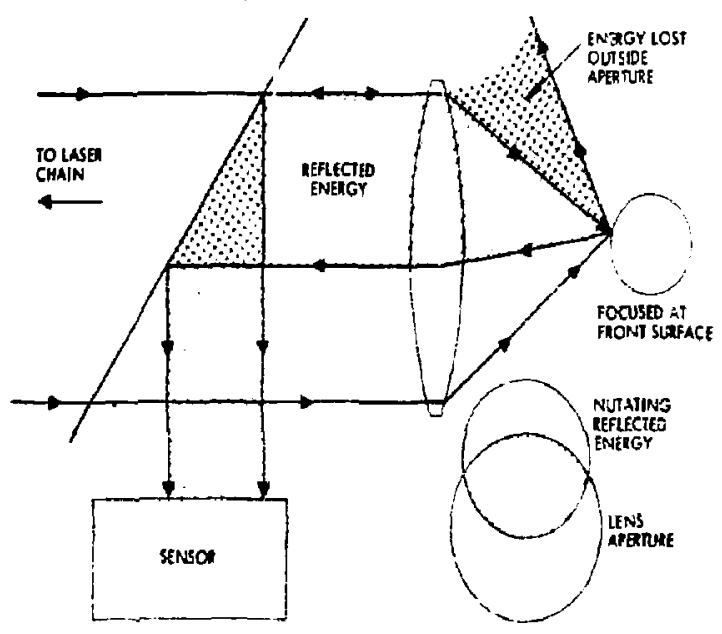

- pirsical descaimtisn

- FOTLSED AT SURFACE OF SHALL 34L

- NUTATVE WTH 30 M CONE ANGIE

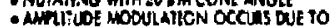

VIGNitiNG

SENST IS ENEEGY-IN-NUCKET TYF

Fipure 11-6. Alignment configuration with focus at surface of small ball 

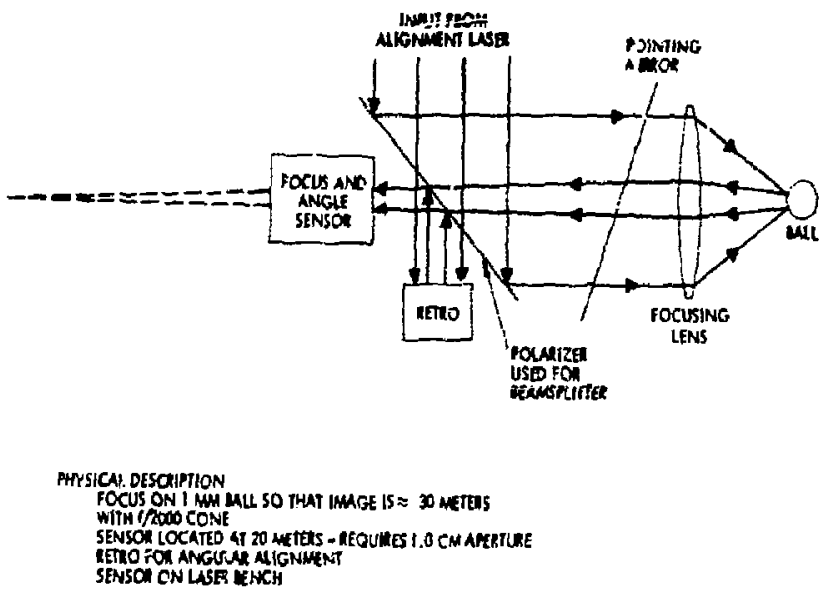

Figure $11-7$, Baseline alignment configuration

the focus sensor reference point could not be precisely located for non-collimated input beam. As for the configuration shown in Figure $11-2$, it was recognized from the statt that the reference pinhole offered the best sensor reference; however, since the alignment beam has to return all the way back to the pinhole, the wayefront of this beam would be twice degraded by the inherent aberrations of the optical train, thereby compounding the degradation of sensor modulation index. Moreover, the presence of any nonaxiallysymmetric aberrations (such as coma) in the system would also introduce correstondingly larger uncertainties in the detection of pointing errors.

In the alignment contigr:ation, Figure $11-2$, the alignment beam is autoreflected from the ball. The sensor is located at the input end of the optical train, close to the pinhole and is referenced to the location of this pinhole.

In the alignment configuration shown in Figure 11-3, the alignment beam is again zutoreflected from the ball. The sensor is located near the locusing lens and is referenced to the irput beam with the beamsplitter and retrorefilector.

In the configuration, Figure $11-4$, the alignment beam is nutated by means of a rotating wedge located near the pinhole. The alignment beam 
reflected from the ball is imaged onto a mask placed at the edge of focusing lens. The nutating alignment beam is modulated by the mask, and is detected by a sensor located near the focusing lens. The sensor is an energy-in-a-bucket type of detector.

In the aligament configuration, Figure $11-5$, the alignment beam after reflection from the ball is brought to an irtermediate focus at the foctsing lens. A sensor lens subsequently relays this image to the detector. The presence of a small central obscuration in the focusing lens requires use of a nutation scheme for the alignment beam.

In the aligmnent configuration, Figure $11-6$, the alignment beam is focused at th. surface of a suitably small ball, and the input beam is nutated. Part of the retuected alignment beam is vignetted by the limiting aperture of the focusing lens, thereby introducing amplitude moduiation into the transmitted portion of the alignment beam. The sensor is an energy-in-a-bucket type of detector.

Figure 11-7 illustrates the selected pointing and focus sensor configuration. After reflection from the sur rogate target and passage through the fccusing lens, the alignment beam is brought to focus at an intermediate distance of approximately 30 meters and with an $\mathrm{f} / \mathrm{no}$. of 2000 . The sensor is located prior to this point and uses a retroreflected beam as an angle and position reference.

Sensitivities to position errors, both angular and translational, are greatly reduced because of the high $\mathrm{f} /$ no. and the size of sensor objective lens is small because of the beam diameter.

\section{Parametric Iradeoffs Based on Ray Tracing}

Ray trace evaluations were performed for different diameters of surrogate target for the baseline configuration, Figure 11-7, to determine values of ti:e following parameters associated with the alignment beam over a range of image distances (back focal lengths as measured from the focusing lens). The parameters in question were:

Alignment beam diameter at focusing lens

Alignment beam $\mathbf{t} /$ no. 


\section{Diffraction spot diameter}

Relative peak irradiance" at diffraction focus

It was shown that, for the baseline configuration, the relative peak irradiance*, computed for collimated input beam with uniform amplitude distribution, was greater than 95 percent for all ball sizes under $25 \mathrm{~mm}$ diameter, and image distances greater thas 22 meters. Ray trace evaluations were first performed using a collimated input beam to the focusing lens, and then later reiterated with a divergent input beam. The dse of a divergent input beam took into consideration the beam matching between the exit aperture of the spatial filter optics ( $20 \mathrm{~cm}$ diameter) and the entrance aperture of the focusing lens $(25 \mathrm{~cm}$ diameter).

Figure 11-8 illustrates additional parameters considered in tradeolf studies for the baseline configuration. The divergent : nput beam is focused onto the ball by the focusing lers. The reflected beam impinges on the focusing lens with a beam footprint of diameter $d_{l}$. The alignment beam emerging irom this lens is intercepted by the sensor lens, with a beam footprint diameter $d_{2}$. This sensor lens then focuses the alignment beam at $I_{2}$ a distance $F_{2}$ from the lens. $I_{3}$ is the ims of the focusing lens as seen by reflection off the ball. $I_{3}$ has a diameter of $d_{3}$ and is located at distance irom $\mathrm{I}_{2}$

The focus and pointing sensors are located between $\mathrm{I}_{3}$ and the sensur lens. If the focusing lens defines the entrance pupil of the alignment system, then $I_{3}$ is the corresponding exit pupil; a lateral displacement of input beam at the focusing lens will theretore be geometrically relayed to the image at $I_{3}$. As described in an appendix of this report, this image cannot, however, be used as a beam ceatering reference.

Part of the parametric tradeoif study was to examine the value of $d_{1}$, $d_{2}, d_{3}, F_{2}$ and $d_{2}$ for different ball diameters and different sensor lenses. Some of the selected alignment systems derived from this tradeoff study were

\footnotetext{
"Relative peak irradiance is defined as the ratio of the peak irradiarze computed at the diffraction focus to that computed for a perfect system.
} 

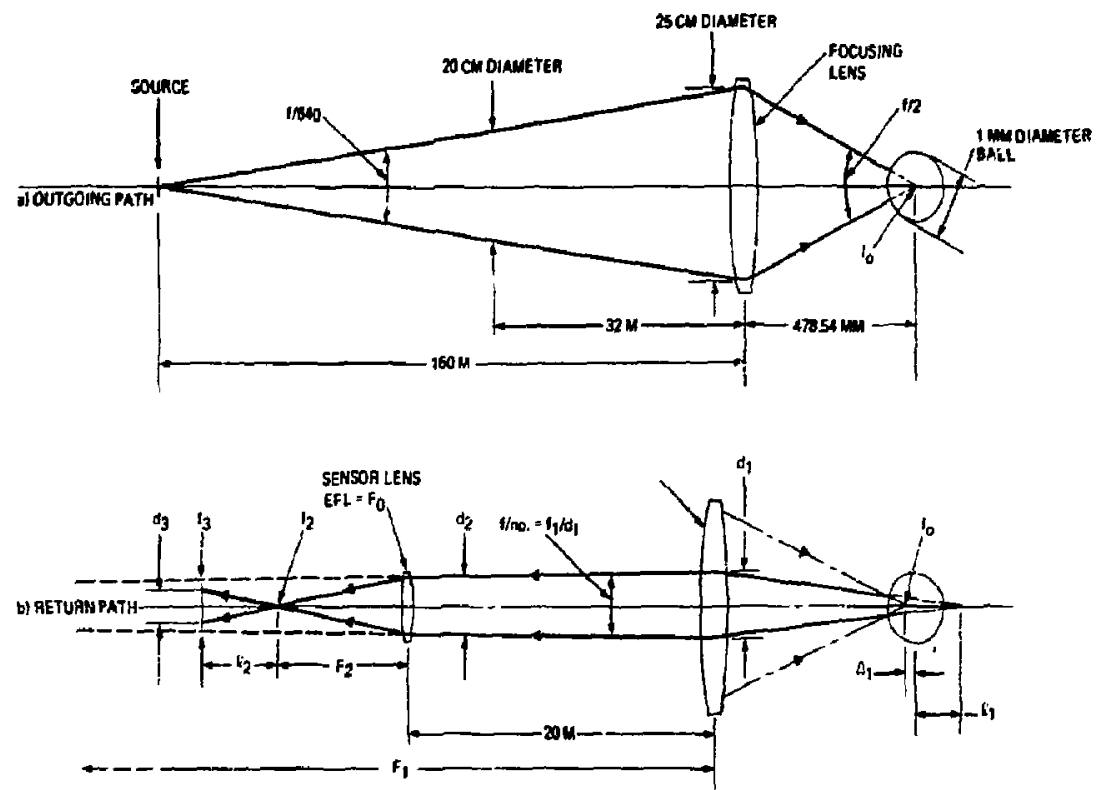

Figure 11-8. Baseline configuration of sensor package and tradeoff study parameters

further analyzed: problems associated with sensor geometry, sensitivity of sensor package to position errors and input wavefront aberrations, initial acquisition, etc. were evaluated under separate tasiks.

\section{SENSOR DESIGN STUDY}

The objectives of this study were:

1. To set up a valid mathematical model for the pointing and focus Bensors

2. To carry out diffraction analyais of alignment systems and to evaluate the susceptibility of sensors to various input wavefront aberrations (diffraction analysis takes into account of the parabolic aperture apodization)

3. To generate point spread and line spread functions for use in the derivation of sensor performance 
Based on data generated in the parametric tradeoff study, three variations (nystems $\mathrm{a}, \mathrm{b}$ and $\mathrm{c}$ ) of the baseline configuration were analyzed. Figure 11-9 depicts the geometrical setup of systems $a, b$ and $c$; the main differences between these systems were that $25 \mathrm{~mm}, 2 \mathrm{~mm}$ and $1 \mathrm{~mm}$ diameter balls were employed, and that the $\mathrm{f} /$ no, and image distance assaciated with the respective alignment beams were different.

The computational details related to the diffraction evaluation are presented in a later Section. Very briefly, the irradiance distribution at or near the image plane is given by tie modulus squared of the Fourier transform of the pupil function. For each optical system (system $3, \mathrm{~b}$ or $\mathrm{c}$ ), the pupil function was evaluated at the exit pupil of the system. This pupil function took into account both the parabolic aperture apodization and the wavefront aberration. The latter included the inherent aberration of the system, and where appropriate, the additional, mathematically simulated input aberrations. The outputs from the diffraction analysis included point spread function, line spread function, knife-edge trace and radial energy distribution,

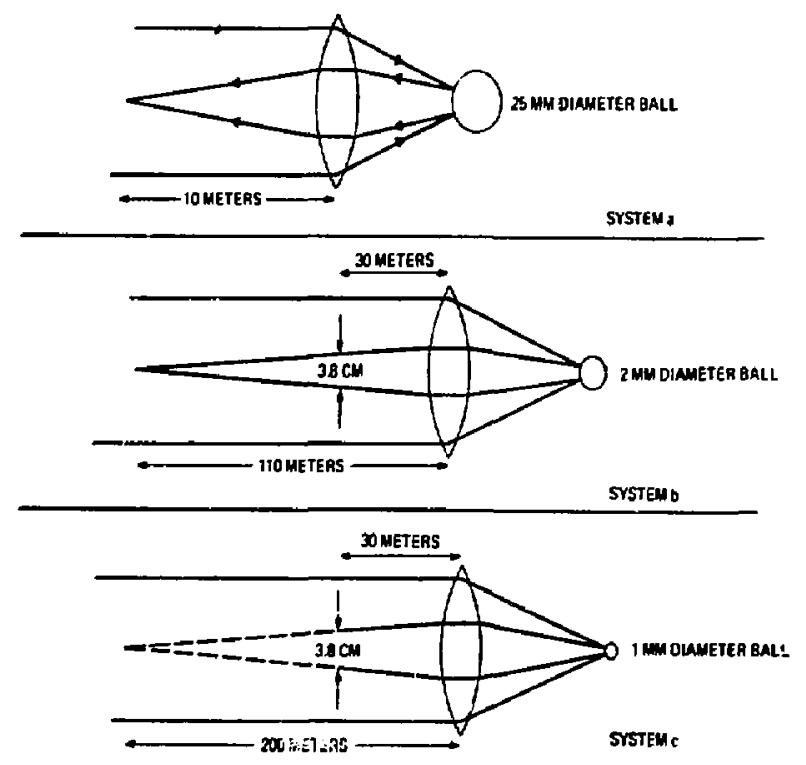

Figure 11-9. Three variations of baseline configuration 
For evaluating the focus sensor, 2 series of through-focus radial energy distributions was first computed. Based on this data, the normalized energies collected by detectors of different sizes were then derived for different defocused image planes. Computational results showed that, due to the parabolic aperture apodization and the presence of aberration, the energy distribution as a function of axial defocusing was asymmetrical about the diffraction locus. Since the proposed focus sensor detects and compares the energy distribution on both sides of the diffraction focus, the instrumental error resulting from asymmetrical energy distribution must therefore be calibrated out by suitably biasing the focus sensor system. Further details related to the focus sensor study are presented in a later Section.

Th: line spread functions and knife-edge traces associated with different input aberrations and different defocused image planes provided useful information for tile pointing sensor design und for calculating the modulation Index of sensors. It is shown in a later Section that, if the aberrated input wavefront contains asymmetrical components (such as coma), this will result in null shift errors in the pointing sensor that can vary whenever the alignmert beam is defocused. This null thift can be expressed in terms of equivalent energy centroid translation at the surrogate target. For system $b$, Figure 11-42 shows the variation of this equivalent translation as a function of defocus error at the surrogate target; the corresponding input waveaberration is one waveleagth $(1.06 \mu \mathrm{m})$ coma peak-to-peak and the focusing lens has an aperture of $\mathrm{f} / 2.0$. Figure $11-42$ shows that the total equivalent translation resulting from this aberration is less than $1 \mu \mathrm{m}$ when the incident beam at the target is defocused through $\pm 7 \mu \mathrm{m}$, the latter being the focus error tolerance.

The effects of equivalent translation on the sensor design will be examined from the system standpoint in a later Section. Further fetails related to the optical aspects of the sensor design study will also be presented in a later section.

Sensitivity Study

The sensor package should be reasonably insensitive to the tolerances of its location and orientation, relative to other system elements. On the other 
hand, since the function of this sensor package is to detect the alignment errors at the target, the package should therefore be highly sensitive to these errors. This sensitivity study is devoted entirely to these considerations as applied to the chosen basline design.

The items investigated are:

i. Axial and lateral movements of the sensor package

2. Axial and lateral movements of the surrogate target, for equivaleridy the focusing lens)

3. Pointing angles of the input beam

For all axial movements, the criterion for sensitivity is the growth in spot diameter on the detectoz. For all lateral movements and pointing angles of the input beam, it is the lateral shift of the 50 percent energy point of this spot, relative to the center of the quadrant detector.

For axial movements, it was found that there is negligible growth of the spot diameter as the sensor package is axially displaced by as much as $\pm 10.0 \mathrm{~mm}$; whereas, the spot diamater doubles when the surrogate target for the focusing lens) is axially displaced by only $\pm 10 \mu \mathrm{m}$. For lateral movements, similar characteristics can be observed. When the sensor package is laterally displaced by $2.0 \mathrm{~mm}$, the lateral shift of the 50 percent energy point on the detector is only $48 \mu \mathrm{m}$ which compares to a lateral displacement of the surrogate target of $2 \mu \mathrm{m}$ and causes a shift as large as $118 \mu \mathrm{m}$. A pointing angle of only 4 mrad causes the 50 percent energy point on the detector to shift laterally by as much as $235 \mu \mathrm{m}$. Further details of this sensitivity study will be presented in a later Section.

\section{CONFIGURATION STUDIES AND TRADEOFFS}

\section{Initial Design Solutions}

The alignment configuration, shown in Figure 11-7, was selected as the baseline configuration for the design study. Further discussions related to the different configurations will be presented in this section. 


\section{Alignment Configuration (Figure 11-2)}

For this alignment configuration, the wavefront of the alignment beam would be twice degraded by the inherent aberrationg: of the optical train, the reby compounding the degradation of sensor modulation index. An example showing why this degradation is not acceptable follows,

It will be shown in this Section later that, if the alignment beam has a wavefront containing asymmetrical components (such as coma), the result is null shift errors in the pointing sensor that can vary as the alignment beam is focused. This null shift can be expressed in terms of equivalent energy centroid translation at the surfogate target. Figure 11-42 shows the variation of this equivalent translation as a function of defocus error at the surrogate target. The wave-aberration of the incident beam at the target ball is one wavelength $(1.06 \mu \mathrm{m})$ coma** peak to peak, and the focusing lens has an aperture of $\mathrm{f} / 2,0$. Figure $11-42$ show 3 the total equivalent translation that results from this aberration is less than $1 \mu \mathrm{m}$ when the incident beam at the target is defocused through $\pm 7 \mu \mathrm{m}$; the latter is the focus error tolerance.

The results shown in Figure 11-42 correspond to the case in which the alignment beam is detected by a sensor located neas the focusing lens (i,e. the sensor-at-pinhole approach is not used here). For this case, $1 \lambda$ coma in the input wavefront will result in $.1 \lambda$ coma in the alignment beam. The resulting $1 \mu \mathrm{m}$ equivalent translation at the surrogate target can be considered as the marginally acceptable error budget for the pointing sensor. However, for the case corresponding to the configuration in Figure 11-2, the sensor-at-pinhole approach requires the alignment beam to return all the way back to the pinhole. The alignment beam will be twice degraded, and the total coma in the alignment beam will now be $2 \lambda$. It is clear that the resulting equivalent translation at the surzogate target must necessarily exceed the $1 \mu \mathrm{m}$ error budget discussed above.

\footnotetext{
*The inherent aberrations of the optical train are not predictable at this time. *** This $1 \lambda$ coma simulates the total odd-component wavefront errof of the input wave after traversing the entire optical train from the reference pinhole to the focusing lens. The magnitude of this abertation is considered to be a worst-case estimate of the quality of the optical train.
} 


\section{Alignment configuration (Figure |1-3)}

As discussed in the Technical Overview above the alignment beam for this configuration is autor eflected of the ball, and the sensor package is located close to the focusing lens (See Figure 11-3). As compared with the configuration in Figure 11- 7 , the alignment beam here must necesarily be much larger. It was pointed out in the Technical Overview that this configuration was rejected becauso it required the use of relatively large optics and, more importantly, the focus senjor zeference point could not be precisely located for noncollimated input beam. In the selected baseline configuration, Figure 11-7, only small and simple optics are required, and the problem associated with focus sensor reference is overcome by using large f/no, alignment beam.

\section{Alignment configurations (Figures 11-4, 11-5, and 11-6)}

It was also pointed out in the technical overview, above, these configurations were rejected because they all required the use of a nutuation scheme. Such a scheme generally introduces additional complications into the system and renders the alignment configuration less atiractive as compared with the simple baseline configuration, Figure 11-7. Moreover, each of these alignment configurations has additional problems which also contributed to its unfavorable disposition. Thus, in the configuration shown in Figure 11-4, the nutated beam incident on the ball would introduce aberrations whenever the beam is displaced off the vertex of the ball. The reflected alignment beam is sensitive to both the aberrations and the focus error. Furthermore, no compatible focus scheme could be found. In the configuration shown in Figure 11-5, the focusing lens acts as a field lens for the returning alignment team. Because this beam forms an intermediate image at the focusing lens, this image location can be ased to provide pointing and focus references, However, with the alignment beam nutated to bypass the small central obscuration in the focusing lens, a large and relativeiy complex sensor lens would be required as compared with the small and simple lens used in the alignment configuration in Figure 11-7.

"requirement as specified by Lawrence Livermore Laboratory. 
In each of the alignment configurations of Figures 11-4, 11-5, and 11-6, the nutated alignment beam is highly sensitive to input wave aberrations containing asymmetrical components such as coma. The presence of such aberrations causes uneven degradation in the nutated aligament beam, resulting in unacceptable pointing errors. It is interesting to note that these errors show up even when the target ball is properly aligned. An example is presented here for the configuration shown in Figure 11-4. The input beam is nutated about the optical axis with an angle of $130 \mu \mathrm{rad}$. It is reflected off the surface of the ball and brought to a focus at the annular mask located at the focusing lens. Figure 11-10 represents schematically the relationship between the annular mask and the positions of the focused spot as the input beam nutates. If the ball is precisely aligned with the center of the annular mask, which is pre-aligned with the optical axis, then the circular locus of the nutating forused spot will be concentric with the annulus. When the ball is decentered, this locus will no longer be concentric and the energy transmitted through the annulus will vary as the input beam nutates. The

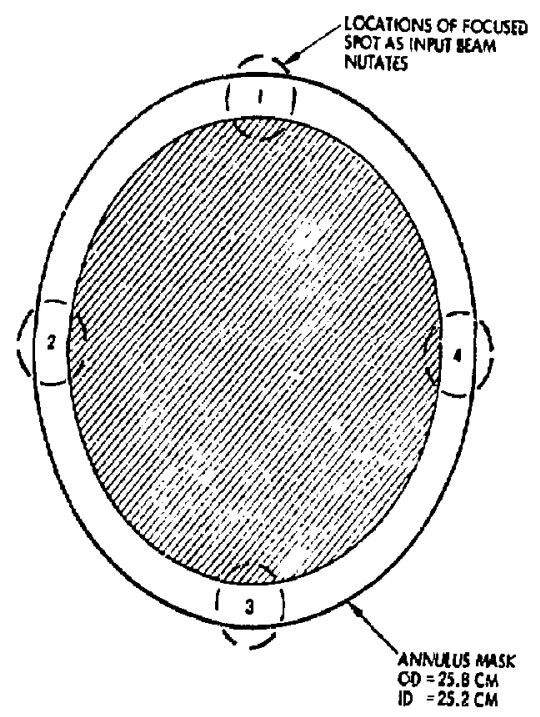

Figure 11-10. Relationship of focused $s$ pot locus with respect to the annulus mask as seen from detector 
minimizing of tinis variation of energy transmitted as the beam nutates, the refore, should be equivalent to centering the ball. As shown in Figure $11-10$, four locations at the mask were examined for transmitted energy as a function of ball decentering. The results are listed in Table $11-1$. For perfect input bearn, it is seen that the variation of transmitted energy at these four locations is quite drastic, even for a ball decentered by only $1 \mu \mathrm{m}$. When the input wavefront contains asymmetrical aberrations $(\lambda / 2$ coma, $1 / 2$ astigmatism) in adeition to $\lambda / 2$ spherical, however, the results are highly unpredictable. Not only do they show similar variations for different degrees of decentering, there is a variation by a factor of two even when the ball is perfectly aligned.

Alignment configuration (Figure 11-7) - the baseline configuration for the design study.

One of the objectives of this design study was to exploit the design possibilities of a potentially good alignment system without the specific need of a deformable mirror. The alignment configuration shown in Figure 11-7, was selected as the final baseline configuration for this design study. Some

Table 11-1. Transmitted energy at four locations on the mask as a function of ball decentering

\begin{tabular}{|c|c|c|c|}
\hline \multirow[b]{2}{*}{$\begin{array}{l}\text { Input Wave } \\
\text { Quality }\end{array}$} & \multirow{2}{*}{$\begin{array}{c}\text { Location } \\
\text { on } \\
\text { Mask }\end{array}$} & \multicolumn{2}{|c|}{ Percent of Input Energy Transmitted } \\
\hline & & $\begin{array}{c}\text { Ball Decentering } \\
\Delta y=0\end{array}$ & $\begin{array}{c}\text { Ball Decentering } \\
\Delta y=1 \mu \mathrm{m}\end{array}$ \\
\hline \multirow{4}{*}{ Perfect } & 1 & 1.000 & 0.360 \\
\hline & 2 & 1.000 & 1.000 \\
\hline & 3 & 1.000 & 0.020 \\
\hline & 4 & 1.000 & 1.000 \\
\hline \multirow{4}{*}{$\begin{array}{l}\lambda / 2 \text { Coma } \\
\lambda / 2 \text { Astigmatism } \\
\lambda / 2 \text { Spherical }\end{array}$} & 1 & 0.348 & 0.460 \\
\hline & 2 & 0.234 & 0.232 \\
\hline & 3 & 0.564 & 0.204 \\
\hline & 4 & 0.234 & 0.232 \\
\hline
\end{tabular}


introductory descriptions of this configization were outlined in the technical overview above. It was also pointed out that this configuration fulfilled the requirements associated with the optical design guidelines discussed in the overview. This matter will now be considered in greater detail.

To facilitate the discussion, a simplified example will be taken from the parametric trade-off study, below. Figure $|l-1|$ illustrates a particular geometric setup for the baseline configuration using a $1 \mathrm{~mm}$ diameter target ball; numerical values associated with the various parameters are exiracted from Table 11-3. Referring to Figure 11-11, the divergent input beam is focused on to the ball by the $f / 2$ focusing lens. The reflected beam impinges on the focusing lens with a beam footprint of diameter $d_{j}$. The alignment beam emerging from this lens is intercepted by the sensor lens, with a beam footprint diameter $\mathrm{d}_{2}$. This sensor lens then focuses the alignment beam at $L_{2}$, a distance $F_{2}$ from the lens. $I_{3}$ is the iniage of the focusing lens as seen by reflection off the ball. $I_{3}$ has a diameter of $d_{3}$ and is located at distance $\ell_{2}$ fr.m $I_{2}$.

The focus and pointing sensors are located between $l_{3}$ and the sensor lens. If the focusing lens defines the estrance pupil of the alignment system, then $I_{3}$ is the corresponding exit pupil; a lat ral displacement of the input beam at the

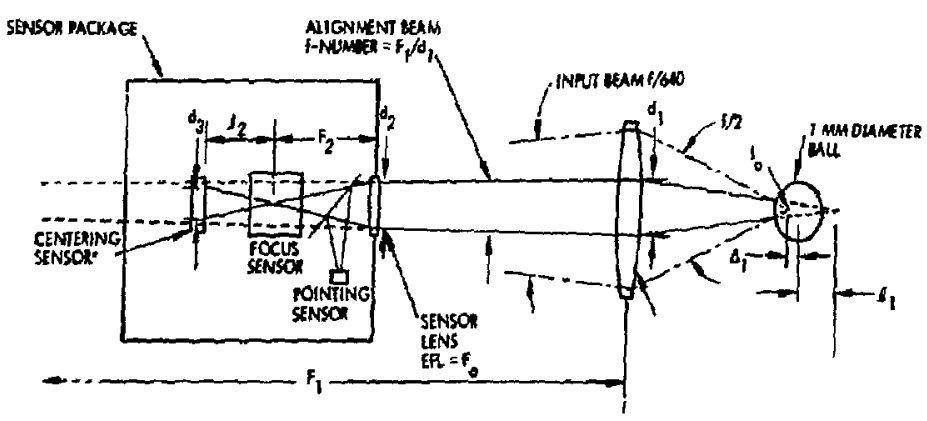

\begin{tabular}{|c|c|c|c|c|c|c|c|c|c|}
\hline $\begin{array}{l}\text { ALIGNNENT } \\
\text { RANUNA }=F_{1} / A_{1}\end{array}$ & $\Delta_{1}$ & $l_{1}$ & $d_{1}$ & $F_{1}$ & $d_{2}$ & $F_{2}$ & $t_{2}$ & $F_{\text {q }}$ & $d_{3}$ \\
\hline 372.2 & $0.25 \%$ & 1.2007 & 85,36 & 5758.0 & 10,100 & 500.0 & $2,05 \mathrm{~s}$ & sot.s & 0.117 \\
\hline
\end{tabular}

DATA DERTD FOMCASE (f) IN TALE X,3,2 (UNITS AN IN UWP)

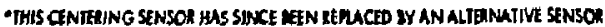
LOCATED AT THE FOCUSING LENS

Figure 11-11. An example of the geometric setup for the baseline configuration 
focusing lens will therefore be geometrically relayed to the image at $I_{3}$ and consequently a sensor at $I_{3}$ can serve as centering sensor: for the input bearn.

Before the focus sensor can be setup to detect the focus error at the target, it is first necessary to locate the sensor reference point relative to which the focus sensor was originally calibrated. By proper utilization of optical leverage, the sensor reference point as measured from the focusing lens can be given reasonably loose tolerances without introducing significant uncertainty errors at the target focus. The esample presented in Figures 11-11 and $1 \mathrm{I}-12$ shows how the sensor reference location is desensitized. Figure $11-12$ (a) shows a simplified version of Figure 11-11, with the sensor package deleted; the $\mathrm{f} / 2$ beam is focused at $I_{0}$ and the tinal image associated with the alignment beam is formed at $I$. Inet $\Delta Z_{0}$ be a small axial displacement of $I_{0}$ and $\Delta Z$ the resulting displacement of $I$. Using first order optics,

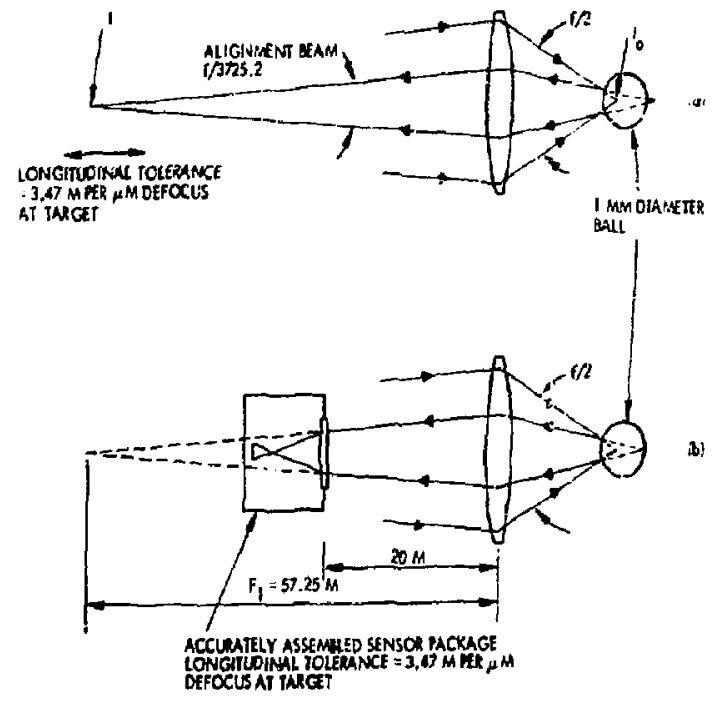

Figure 11-12. A simplified version of the example shown in Figure 11-11

\footnotetext{
*As described in an appendix of this report, an alternative centering sensor scheme is used in the finalized alignment contiguration.
} 
the ratio $\Delta Z / \Delta Z_{0}$ is related to the $l / n o . s$ of the input and the alignment beams by the equation

$$
\frac{\Delta Z}{\Delta Z_{Q}}=\left(\frac{f \text {-nuriber of alignment beam }}{\text { f-number of input beam }}\right)^{2}
$$

For the case snown in Figure 11-12, the $f$ 'no, of the input beam at the target is $i / 2$, and that of the slignmient beam is $f / 3725.24$. The corresponding value of $\triangle Z / \Delta Z_{0}$ is therefore

$$
\begin{aligned}
\frac{\Delta Z}{\Delta Z_{\mathrm{g}}} & =\left(\frac{3725.24}{2}\right)^{2} \\
& =3.47 \times 10^{6}
\end{aligned}
$$

Thus, if $\Delta Z_{0}=1 \mu \mathrm{m}$, then $\Delta Z=3.47$ or $3.47 \mathrm{~m}$ per $\mu \mathrm{m}$ iucus error at the targei. In other words, within the approximations of first order optics, an error of $3.47 \mathrm{~m}$ in the axial location of [ would he equiralent $10 \mathrm{i} \mu \mathrm{m}$ defocus of the $\mathrm{t} / 2$ beam at the ball, implying that, for the case shown in Figure 11-11, a loose tolerance can be assigned to the focus sensor reference.

In practice, a reaístic longitudinal tolerance $\sigma$, say, $\pm 0.5 \mathrm{~m}$ can be specified as the location error for positioning the : xcus sensor relative to the focusing lens. For the exaryple given in Figure $1 /-11$, the sensor reierence point, which also defines the norsinal location of the focus senşor unit, is $20 \mathrm{~m}$ from the focusing lens. If the focus sensor unit is accurately assembled (shown as $a$ box in Figure $11-12 b$ ), then the tolerance for $\Delta \mathrm{Z}$ as computed from Eq. (11-1) for the longitudinal displecement of the image point I will also define the longitudinal tolere nce for the sensor unit. Thus, if the sensor unit has a position error of $\pm 0.5 \mathrm{~m}$ relative to the sensor reference point, then the resultirg focus error at the target would $b a \pm(0,5 / 3,47$ $\left.\times 10^{6}\right)= \pm 0.14 \mathrm{um}$, which is negligible. In othei words, provided the focus sensor wiit is accurately assembled*, the sensor can be placed on the laser bench with $\pm 0.5 \mathrm{~m}$ position error relative to the sensor reference point without introducing noticeable focus error at the target. The large optical leverage associated with the configuration shown in Figure 11-7 makes it

For the case under consideration, an accurately assembled focus sensor fulfills the imaging requirements as defined in Figure 11-11. 
possible fo: the focus sensor to have loose longitudinal tole rance on its lockit:r relative to the target without degrading the detectabiity of focus errors at the teiget

It will be: show'n belory that she baseline configuration (Figure $11-7$ ) also ailows reasorable tolerances on sinsor translation and sensor angular stability. In view of the smail diameter oi the alignment beam (see Figure 11-11, and that the $\mathrm{f} / \mathrm{ho}$. of the sensor lens is of the vitar of $\mathrm{f} / 50$ :0 $\mathrm{f} / 100$, the sensor packagc requires only small and simple optir:. As shown beiow, the basic geometrical setup of the alignment configuration sicun in Figure 11-7 has negligible spherical aberration, and for all practical purpises, this alignment configuration does not further degrade the input wavefr:nt.

Additional details related to the alignment and acquisition proceniures for this bazeline configuration are presented in this Section, as are sen:ors for focus, pointing and centering detection. Finally, a computer nodel for the pointing and focusing sensors is ciescribed in which the susceptibility of sensors to various input wavefront aberrations is also studied.

Parametric Tradeoffs Based on Ray Tracing

The evaluation of difie rent geometrical setups of the baseline configuration are described in this subsection.

\section{Two Baseline Configuration Versions}

The baseline design configuration chosen and described in the previous subsection was given in two ver sions: 1) for collimated input beam, as described in Figure 11-7 and(2) for divergent input beam, as described in Figure 11-8.

For the version with collimated input beam, an $t / 2$ focusing iens of 500. mm equivalent focal length (EFL) was used; for that with divergent input beam the lens used was $\mathrm{f} / 1.586$ with $395.45 \mathrm{~mm}$ EFL. Each of these lenses was aspherized to minimize the spherical aberration at the target plane, A complete description of the characteristics of focusing lenses are listed in Tabie 11-2. 
Table 1:-2. Characteristics of focusing lenses used in baseline configuration

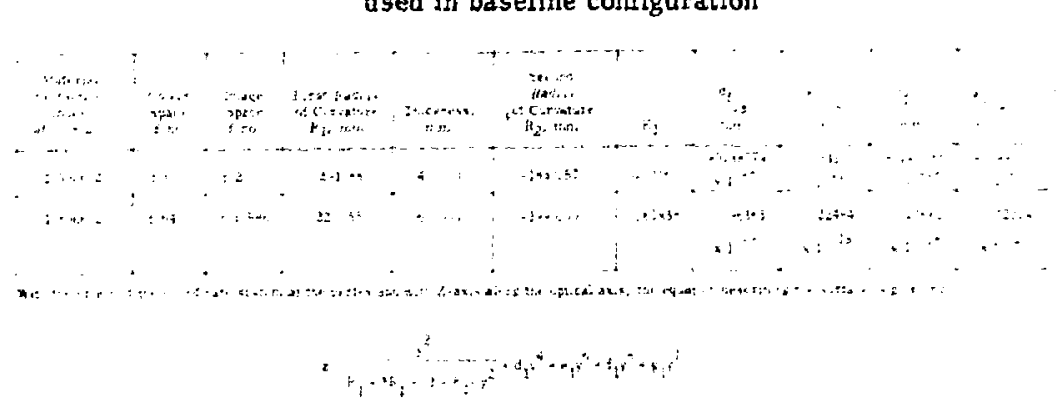

\section{Description of Parameters Involved in Tradeoff Study}

Since the surrogate target is effectively a mirror, the spot focused by the focusing lens is ro-imaged $b y$ it. The new image, in turn, serves as an object for the focising lens on the return path. By moving the focusing lens along the optcal axis, the returning beam (or the alignment beam) can be manipulated in terms of its $\mathrm{f} / \mathrm{no}$., image distance and beam diameter at various lonations of interest. In other words, a parameter tradeoff study can be performed.

For convenience, these parameters are arbitrarily divided inta two groups: irdependent and dependent parameters. The independent parameters include the ball diameter, ${ }_{B}$, image distance, ${ }_{i} ;$ and, at later stages of the study, the diameter, $d_{2}$, of the returning beam at the sensor lens and its focal disiance, $F_{2}$. (See Figure 11-11.) The dependent parameters include the $f / r: o_{*}, f_{1} / d_{1}$, of the returning beam as it leaves the focusing iens, bean diameters at the focusing lens, $d_{1}$, and at the sensor lens. $d_{2}$; and, in later stages, the distance, $l_{2}$, between the focus sersor and the exit pupil of the system, and, finally, the diamete: $d_{3}$ at the exit pupil. The exit pupil oi the system, where the centering sensor is locate ${ }^{*}$, is the image of the focusing lens as reflected off the surrogate target and refracted through the focusing and gensor lenses.

\#As described in the appendix of this report, an alternative centering sensor is uged for the fitalized alignment configuration. 
In addition to the dependent parameters mentioned above, some $\varepsilon / n o$. ielated parameters such as the diffraction spot diameter and its relative peais irradiance, RPI, were also examined in the early stages of the tradcoff study.

Throughout the tradecff study, trigonometric ray tracing was employed to compute the parameters. Parayial ray tracing, though helpful, was inadequate because the focusing lenses are of low $\mathrm{f} / \mathrm{ho}$, and highly corrected for spherical aberration at the target plane.

\section{Tradeaff Study Sequence and Results}

Collimated Inpit Beam - The tradeofis performed for this case are primarily concerned with the effects of ball diameters, $d_{j y}$ and image distances. $F_{1}$, on the size of the sensor package and image quality of the returning beam, which is of cuurse related to its $\mathrm{f} / \mathrm{no}$. The results are given in Figures 1 :-13 through :1-16.

From Figure : $1-\$ 3$, it is seen that $l\}$ the beam diameter, $d_{j}$ at the focusing lens increases as the image distance, $F_{1}$, ircreases, and 2) $d_{1}$ cecreases for decreasing ball diameters. Figure 11-14 shows that, as a function of image distance, the $f / n o$. of the returning beam remains fairly constant, but it is inversely proportional to the ball diameter. The consequence is shown in Figure 11-15 the diffraction spot diameter increases with derreasing ball diameter. The diffraction spot diameters were computed sccording to

$$
d=2.3 \hat{8} \times \lambda \times t / n o, \text { where } \lambda=1.06 \mu \mathrm{m}
$$

These are, of course, theoretical limits for a parabolically apodized input beam intensity. Eor real systems, the relative, eak irradiance (RPI) as defined above serves as a measure of how closely it approaches its theoretica1 limit. Figure 11-16 shows that, for image distances beyond 22 metexs, the RPI is above 95 percent for all ball diameters less tnan $25 \mathrm{~mm}$.

Divergent Input Beam - As the tradeoff study proceeded from this point, the degree of divergence given and the focusing lens used underwent some changes. These changes, however, did not alter the general characteristics of the results. 


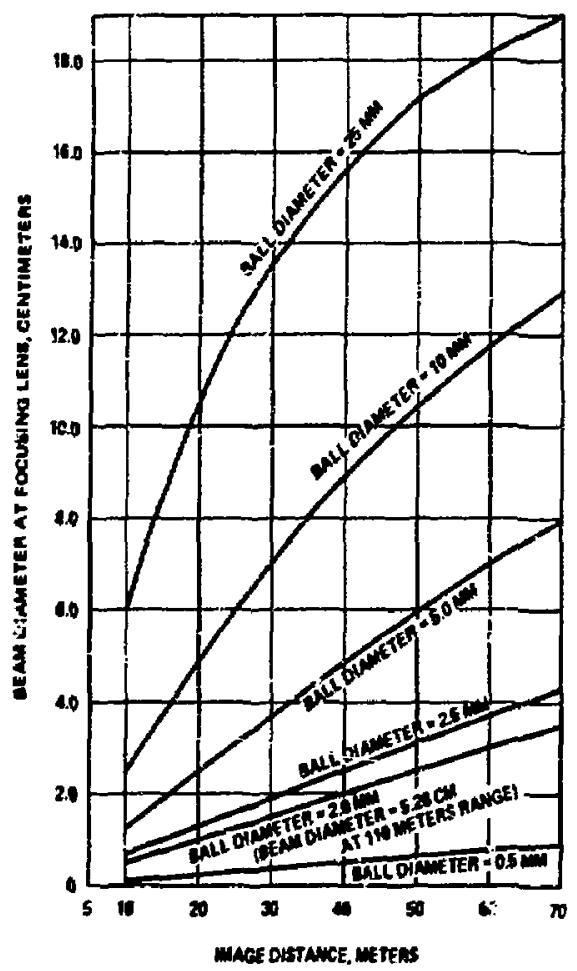

Figure 11-13. Bea;n diameter al focusing lens as a function of image distance for various ball diameters 


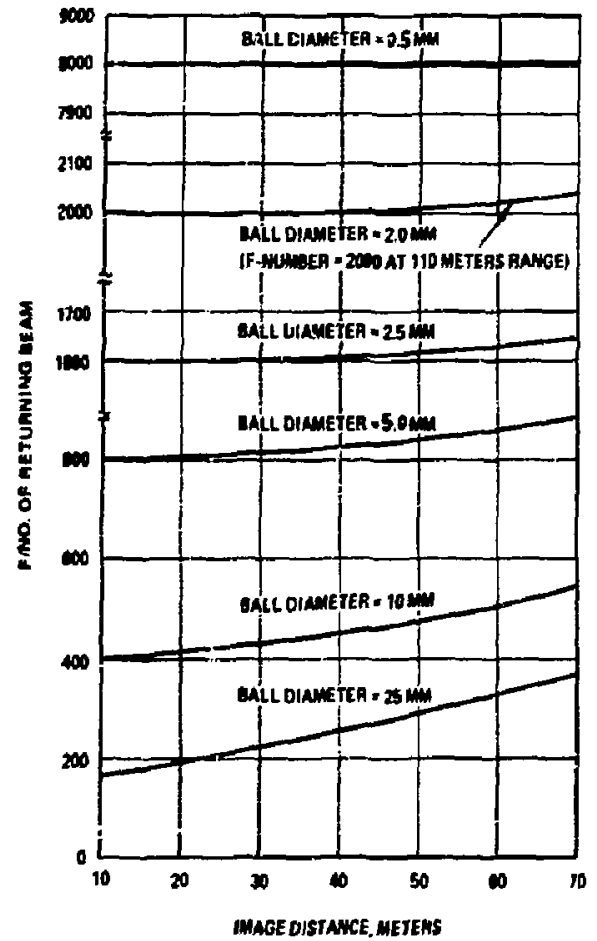

Figure $11-14$. F/no, as a function of image distance for various ball diameters 


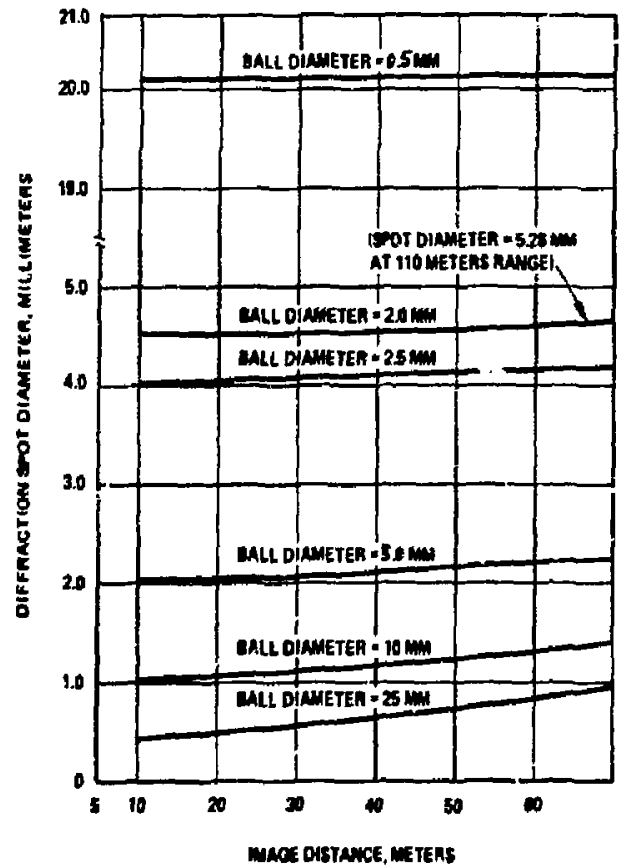

Figure 11-15. Ciffsaction spot diameter as a function at image distance for various ball diameters 


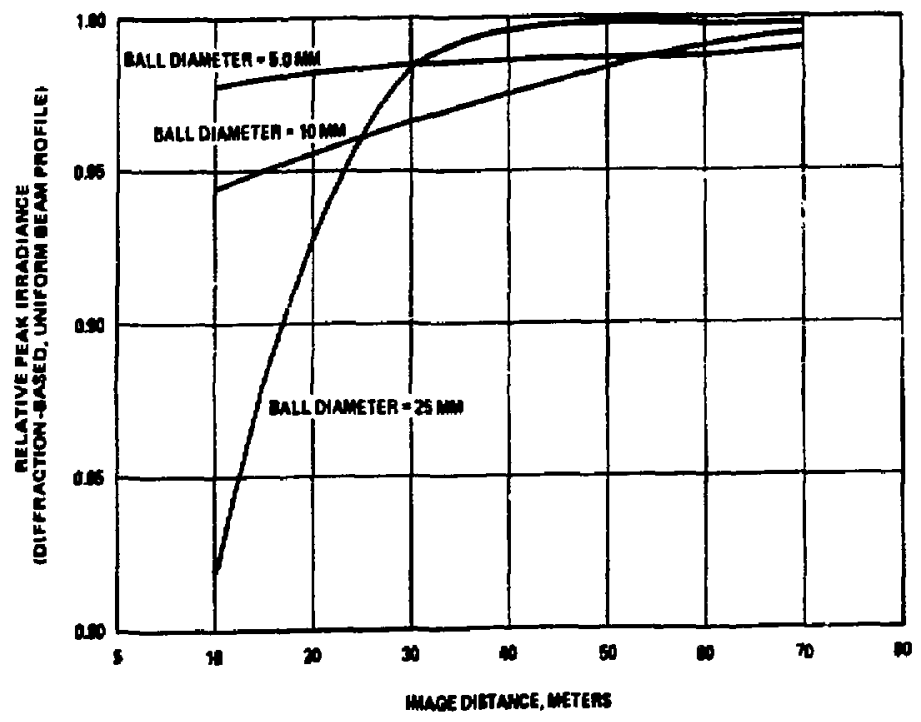

Figute 11-16. Relative peak irradiance as a function of image distance for variouy ball diameters

Originally, the degree of divergence of the input beam was $\mathrm{f} / 245$, with the equivalent point source located at 61.25 meters from the focusing lens. This was the same lens used in the collimated beam case. Figires $11-17$ through 11-19 were obtained under these conditions. They are similar to Figures 11-13 through 11-15 and, therefore, similar conclusions can be drawn from them. From considerations of the size of sensor package, available space, and sensitivity of the sensor package to the alignment errors, a $1.0 \mathrm{~mm}$ diameter was chosen and the distance between the focusing lens and the sensor lens was fixed at 20 meters.

At this stage, the input beam divergence was changed to $f / 640$ with the equivalent point source located at 160 meters from the focusing lens. The independent parameters for this phase are the diameter, $d_{2}$, at the sensor lens, and its focal distance, $F_{2}$. The results are listed as cases $(A)$ through (F) in Table 11-3. In this table, the symbols are identical to those used in Figure 11-8.

Finally, the $f / 2$ focusing lens was replaced by the $f / 1.586$ lens, and results are listed as cases (G) and (H) in Table 11-3. 


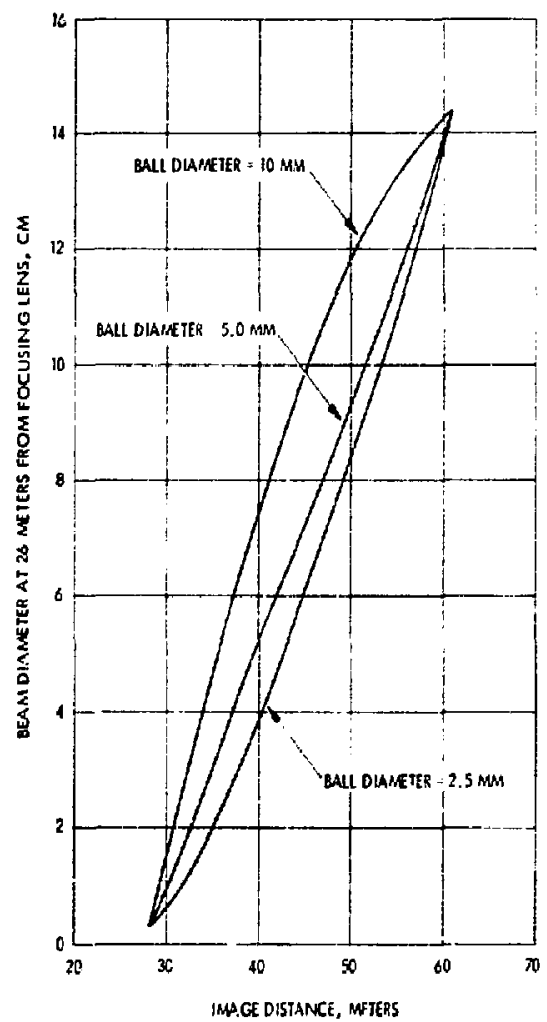

Figure 11-17. Beam diameter at 26 meters from focusing lens as a function of image distance for various ball diameters 


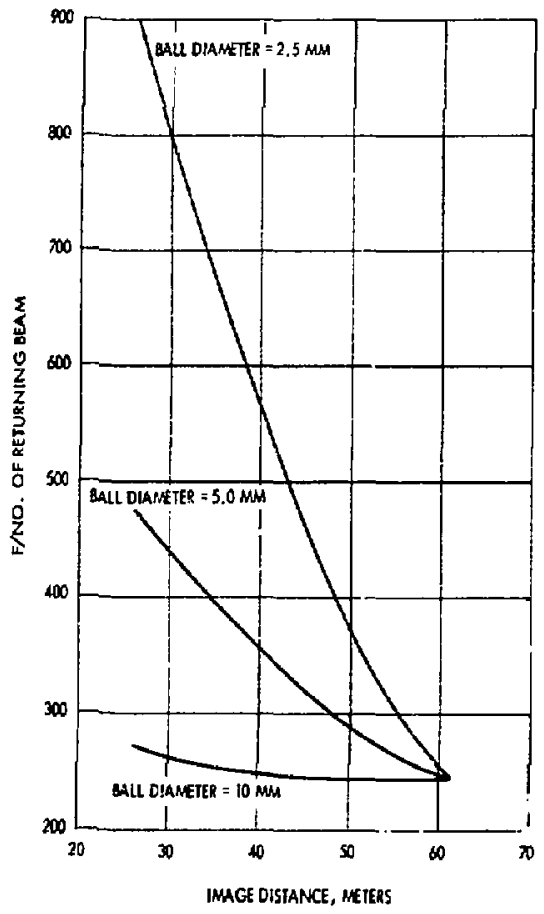

Figure 11-18. F-number as a function of image distance for various ball diameters 


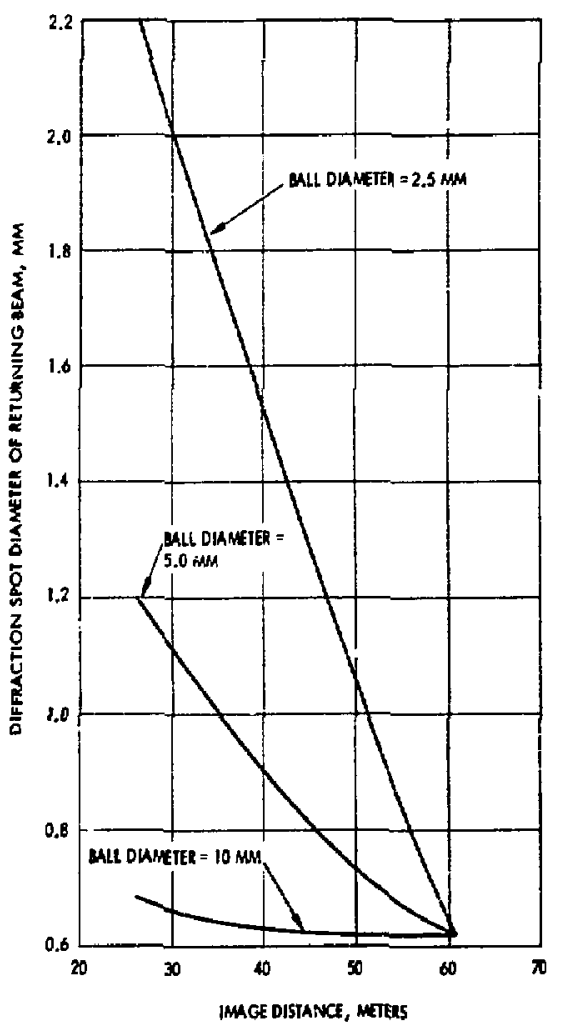

Figure 11-19. Diffraction spot diameter as a function of image distance for various ball diarseters 
Table 11-3. Results of parametrij tradeoffs with f/640 input beam divergence,

$1.0 \mathrm{cmin}$ diameter ball (For cases (A) through (F), tho focusing

lens used is $f / 2$; for cases (G) and (H), it is $f / 1.586$. )

\begin{tabular}{|c|c|c|c|c|c|c|c|c|c|c|c|}
\hline Cases & $A_{10}$ & in. & $\begin{array}{l}\mathbf{u}_{1} . \\
\text { minn }\end{array}$ & $\begin{array}{l}\text { Nivers } \\
\text { melers }\end{array}$ & $\begin{array}{c}f=\text { number. } \\
F_{1} / d_{1}\end{array}$ & $\mathbb{m}_{2}$ & $\begin{array}{l}F 2 \cdot \\
m+1\end{array}$ & us & ${ }_{2}$ & ${ }_{m}^{k}$ & $\begin{array}{l}\text { dis, } \\
\text { mn }\end{array}$ \\
\hline (A) $\begin{aligned} d_{2} & =3 \mathrm{dm} \\
k_{2} & =100 \mathrm{~d}_{2}\end{aligned}$ & 13.2124 & 1.3344 & 34,742 & $\$ 1.277$ & $213+2.5$ & 318. 1406 & $313(x, n$ & 0.0761 & $54.61 \%$ & 3119.8 & 0.5972 \\
\hline (B) $\begin{aligned} d_{2} & =3 \mathrm{~cm} \\
F_{2} & =50 d_{2}\end{aligned}$ & 0.2124 & $1.33+1$ & 317.712 & $81.27 \%$ & $20+2.5$ & 30.0000 & 1500.0 & 0.03 .98 & 13.532 & 1527.2 & 0.2981 \\
\hline (c) $\begin{aligned} d_{2} & =2 \mathrm{~cm} \\
F_{2} & =130 d_{2}\end{aligned}$ & 0.2237 & 1. 96,65 & $28 .+64$ & $63.3 \mathrm{BH}$ & 2367.2 & $211,01,3$ & 2007.5 & 0.0987? & 310.931 & 2067.5 & 0. 3087 \\
\hline (D) $\begin{aligned} d_{2} & =2 \mathrm{~cm} \\
F_{2} & =50 d_{2}\end{aligned}$ & 0.2237 & 1. 9665 & 28.468 & 67.358 & 2367.2 & 20.027 & 1000.8 & 1). 05uz & 7.6285 & 1015.5 & 0.2031 \\
\hline (E) $\begin{aligned} d_{2} & =1 \mathrm{~cm} \\
F_{2} & =100 d_{2}\end{aligned}$ & 0.2370 & 4.2037 & $15.36 \%$ & 57. 252 & 3725.2 & (10. & Ju100, o & 1. $16 \mathrm{HS}$ & 11.5114 & 1018.7 & 0.2566 \\
\hline (F) $\begin{aligned} d_{2} & =1 \mathrm{~cm} \\
F_{2} & =50 \mathrm{~d}_{2}\end{aligned}$ & 0.2370 & 4,21937 & 15.369 & 57.252 & 3725.2 & 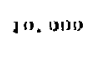 & 500.0 & 0.0743 & 2. 4546 & 504.63 & 0.1176 \\
\hline (a) $\begin{aligned} d_{2} & =2 \mathrm{~cm} \\
v_{2} & =b 1) d_{2}\end{aligned}$ & 0.223 , & 1. inest & $32 . \cdot 77$ & $5,3.3 \times H$ & 166.0. & $20,(1)(1)+1$ & 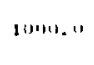 & $12,196,1646$ & 27.252 & $1033+6$ & $0 .+735$ \\
\hline (t) $\begin{aligned} w_{2} & =1 \mathrm{~cm} \\
F_{2} & =5 \times d_{2}\end{aligned}$ & $0.233 \mathrm{i}$ & 2.453 & 20,81 & Bis. 83 & $131 ; 3.6$ & $\mid t i, t w n\}$ & b.the." & 11,0853 & 16.3110 & 5218.18 & (1.2381 \\
\hline
\end{tabular}




\section{SENSOR DESIGN STUDY}

\section{Image Evaluations}

The objectives of the sensor design study have been outlined in the Technical Overview, In this section, diffraction evaluation of three variations of the baseline alignment configuration will be discussed and the susceptability of sensors to different inptt wavefront aberrations will be analyzed. Parts of the diffraction output generated in this study, suck as point spread and line spread functions, are to be used for the purpose of providing reference data to the theoretica: derviation of sensor performance.

The three variations of the baseline configurations, namely systems a, $b$ and $\varepsilon$ in Figure 11-9, were first selested from data generated in the parametric tradeoff study (see Figure 11-13). Of these three systems; only system b was analyzed in detail. The other two systems were evaluated only for providing reference performance data. System a used $25 \mathrm{~mm}$ ball target, and the image distance as measured from the focusing lens was 10 meters. Systems $b$ and $c$ used $2 \mathrm{~mm}$ and $1 \mathrm{~mm}$ balls, respectively. These two systems were set up to har: identicai aignment beam diameters of $3.8 \mathrm{~cm}$ at $30 \mathrm{~cm}$ from the focusing lens. The inac'e distances for systems and b were 110 and 200 meters, respectively. I: all systems, the input beams were taken to be collimated. Non-collimated inputs were not consider ed because they would not cristribute any acititional useful information to the sensor design study. The fecusing lens shown in Figure 11-9 was the f/2 aspheric lens describe in Table 11-2.

Computational details related to the diffraction evaluation are presented in the following subsection. Very briefly, the irradiance distribution at or near the image plane is given by the modulus squared of the Fourier transform of tre pupil funtion. For each of the systems $a, b$ and $c$, the pupil function was evaluated at the exit pupil of the system and took into account both the parabolic aperture apodization as weil as the wavefront aberration. The latter included the inherent aberration of the system, and, where appropriate, the additional mathematically simulated input abersations. The outputs from the diffraction analysis included point spread function, line spread function, knife. edge trace and radial en' rgy distribution. 


\section{Diffraction Evaluation Approach}

\section{Laser Input Beam}

The aperture irradiance function $I(r)$, associated with the high-power laser beam at the focusing lens is $g$ : ven by

$$
\begin{array}{rlrl}
I(r) & =I,\left[1-r^{2}\right]+\frac{I_{0}}{50} \exp \left\{-r^{-5}\right\}, & r & \leq 1 \\
& =0, & r>1
\end{array}
$$

where $I_{0}$ is the peak irradiarce at $r=0$, and $r$ is the relative polar radius. The value of $r$ is calculated from the relative pupil coordinates $(x, y)$ by

$$
r=+\left(x^{2}+y^{2}\right)^{1 / 2},
$$

with $I=1$ at the limiting aperture of the focusing lens. The nominal aperture diameter of the latter is $25 \mathrm{~cm}$. An isometric view of the aperture irradiance function is shown in Figure 11-20.

\section{Diffraction Formulation}

The emergent wavefront at the exit pupil of the alignment system will be associated with a pupil function $f(x, y)$ defined by

$$
f(x, y)=C(x, y) \exp \left[i \frac{2 \pi}{h} W(x, y)\right]
$$

Where $C(x, y)$ is the amplitude of the wavefront at the point $(x, y)$ in the exit pupil; $(x, y)$ are tis rulative pupil coordinates as defined in Eq. (11-3)*; $\lambda$ is the ravelength $(=1.06 \mu \mathrm{m})$, and $W(x, y)$ is the wave aberration of the system. $C(x, y)$ is geometrically related to the square root of the aperture irradiance function $I\left(x_{8} y\right)$ - see Eqs.. 11-2 and 11-3, and has the value zero for points cutside the cxit pupil. $W(x, y)$ includes both the inherent

* Note that, for collimated input beam, the relative pupi! coordinates $(x, y)$ associated with the input beam at the focusing lens are identical to the relative pupil coerdinates at the exit pupil. 


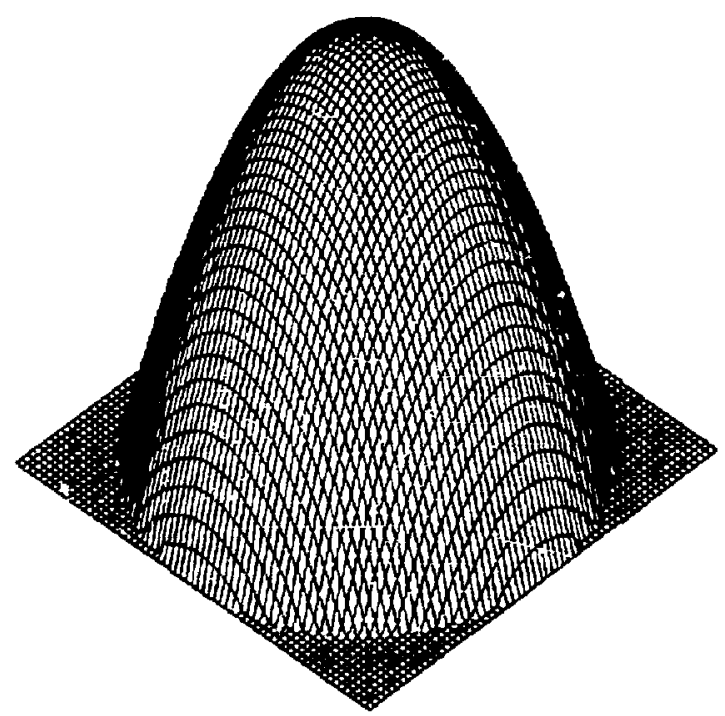

Figure 11-20. Isometric view of the aperture irradiance function

a'serraiions that are present in the systems as shown in Figure $11-28$, and the wavefront aberration of the input beam. Using the Fraunhofer diffraction integ;al, the scalar wave complex amplitude $E(X, Y)$ of the diffracted beam at the image point $(X, Y)$, a distance $d$ from the exit pupil, is gi:: en by

$$
F(X, Y)=\frac{2 \pi}{\lambda d} \int_{-\infty}^{\infty} f(x, y) \exp \left[i \frac{2 \pi}{\lambda d}(x X+y Y)\right] d x d y
$$

Where a phase facto: $\exp \left[i(\right.$ constant $\left.)\left(X^{2}+Y^{2}\right)\right]$ has been omitted on the right hand side.

The irradiance $H(X, Y)$ of the difiracted beam is defined as

$$
H(X, Y)=|F(X, Y)|^{2}
$$


In the image plane, the radial energy distribution ( $R E D$ ) is defined as the energy passing through a circle of radius $b$, centered at the reference point $\left(X_{0}, Y_{0}\right)$, and is given by

$$
\operatorname{RED}(\zeta, \mathrm{d})={\underset{\mathrm{X}}{\mathrm{I}_{-}}}_{\mathrm{Y}_{-}^{+}}^{\mathrm{X}_{+}} \mathrm{H}(\mathrm{X}, \mathrm{Y}) \mathrm{dXdY}
$$

Where

$$
\begin{array}{ll}
Y_{+}=Y_{0}+\zeta & Y_{-}=Y_{0}-\zeta \\
X_{+}=+\left[\zeta^{2}-\left(Y-\Psi_{0}\right)^{2}\right]^{1 / 2}+X_{0} & X_{-}=-\left[\zeta^{2}-\left(Y-Y_{0}\right)^{2}\right]^{1 / 2}+X_{0}
\end{array}
$$

The knife-edge trace $\left(K_{\mathrm{V}} E T\right)$ along $X$-aris, up to the line $X=\alpha$, is given by

$$
\operatorname{KET}(\alpha)=\int_{Y=-\infty}^{Y=\infty} \underset{X=-\infty}{X=\alpha} H(X, Y) d X d Y
$$

Likewise, the knife-edge trace along the $\mathrm{Y}$-axis up to the line $\mathrm{Y}=\alpha$ may be be computed from an equation similar to Eq. (11-9), but with the ranges of integration for $X$ and $Y$ interchanged.

The line spread function (LSF) along the $X$ - and $Y$ - axis are zespectiveiy given by

$$
\operatorname{LSF}(X)=\int_{-\infty}^{\infty} H(X, Y) d Y
$$

and.

$$
\operatorname{LSF}(\mathrm{Y})=\int_{-\infty}^{\infty} H(X, Y) d X
$$




\section{Wave-Aberration Function}

In order to study the susceptability of sensors to different input wavefront aberrations, it is first recessary to modify the plane wavefront of the collimated input beam. The plane wayefront can be deformed by a ware abetration function of the form

$$
w(x, y)=w_{22^{2}}\left(x^{2}-y^{2}\right)+w_{31}\left(x^{2}+y^{2}\right) y+w_{40}\left(x^{2}+y^{2}\right)^{2}
$$

Where

$$
\begin{aligned}
& W_{22}=\text { third order astigmatism } * \\
& w_{31}=\text { third order circular coma* } \\
& w_{40}=\text { third order spinerical aberration }
\end{aligned}
$$

The resultant wave aberration $W(x, y)$ in the pupil furction, Eq. $(11-4\}$, is then defined as the sum of the input wave aberration given by Eq. (11-12) and the inherent wave aberration of the system. The different values of input aberration used in the actual diffraction evaluation are discussed next.

\section{Computational Approach}

The evaluation of the complex amplitude distribution $F(X, Y), E q .(1)-5)$, can be carried out by a two-dimensicnal Fourier transform techrique ${ }^{2}$, operating on the pupil function $f(x, y), E q$. (11-4). The number of sampling points required for this purpose is $64 \times 64$, with the points mapped over the exit pupil.

The two-dimensional irradiance function, $\mathrm{H}(\mathrm{X}, \mathrm{Y})$, can then be computs $;$ from Eq. $(11-6)$, and a plot of $H(X, Y)$ gives the point spread tinction. of : se diffration pattern. The values of radial energy distribution, knifeedge traces and line spread functions are derived from $H(X, Y)$ by numerical integration, using Eq. (11-7) through (11-11).

\footnotetext{
"By definition of Eq. $(11-12)$ : the values of $W_{22}$ and $W_{31}$ will botl be given by cne half of the peak-to-peak wave aberration values. The astizmatism ae defined in Eq. $(11-12)$ correspond s to the case in whish the orthogonal sectioss of the wavef ront have equal and opposite curvatures.

t: The use of Gucrtzel-Wertz algorithm is particularly suitable for this purpose.
} 
Diffraction Evaluation of the Baseline Configuration

The flow chart presented in Figure 11-21 shows the basic steps performed in the diffraction evaluation of system $b$. The different aberration values uged to simulate the input wavefront were defined by cases 1 through 6 in Table 11-4. For each case, a series of through focus diffraction evaluation was first carried out; based on the data generated from the radial energy distribution at different image planes, the energy collected by detectors of different sizes were then detarmined as a function of defocus. The entire computational procedure was repeated for the next case of input aberration until all six cases in Table 11-4 were sudied.

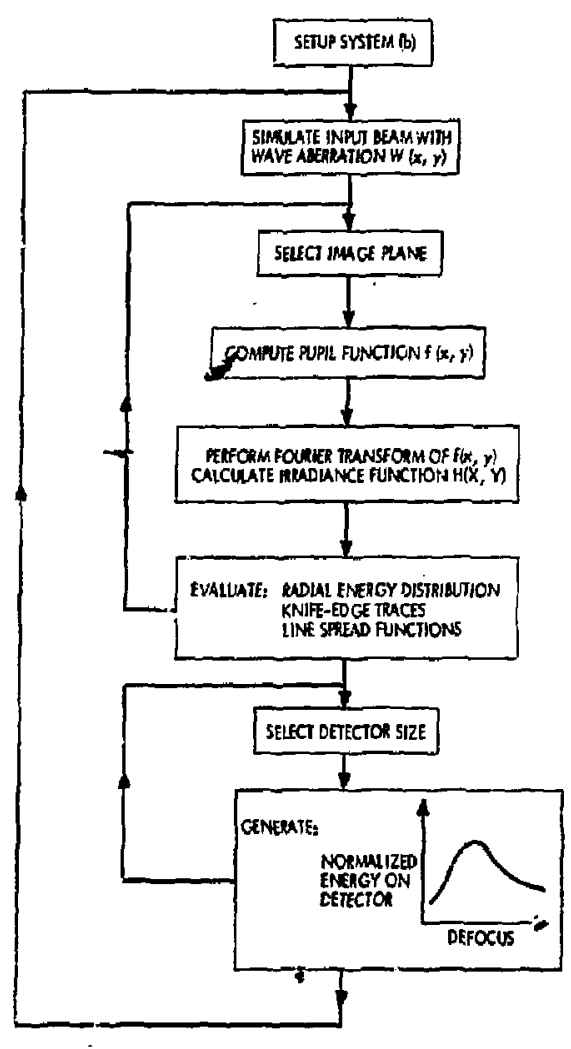

Figure 11-21. Flow chart for diffraction evaluation of system (b) 
Table 11-4. Aberration values for simulating the input wavefront

\begin{tabular}{|c|c|c|c|}
\hline \multirow{2}{*}{ Case } & \multicolumn{3}{|c|}{ Aberration Value (peak-to-pea. ) , $\lambda$} \\
\cline { 2 - 4 } 1 & Astigmatism & Coma & Spherical \\
2 & 0 & 0 & 0 \\
3 & $1 / 2$ & 0 & 0 \\
4 & 1 & 0 & 0 \\
5 & 0 & $1 / 2$ & 0 \\
6 & 0 & 1 & 0 \\
\hline
\end{tabular}

As mentioned earlier in this Section, of the three systems $a, b$ and $c$, only system b was analyzed in detail; the other two systems were evaluated for the purpose of providing reference performance data. The computational output will now be presented below.

\section{Computational Results and General Discussion}

It has been pointed out earlier that the objectives of the diffraction evaluation were to study the susceptability of sensors to different input wavefront aberrations and to provide reference data to the theoretical derivation of sensor performance. It is therefore appropriate to present first a preliminary description of the computational results, including an outline of the information that can be derived from these data; this will then be followed by a detailed discussion of the graphical results.

\section{Preliminary Description of Computational Results}

\section{Normalized Energy Incident on Detectors of Differen' Sizes and at} Different Defocused Image Planes, in Presence of Different Input Wave Aberrations - Based on data generated from the radial energy distribution plcts for different aberrations and at different defocused image planes, the energy incident on focus detectors of different gizes can be evaluated. Graphical plots of normalized energy on different detectors versus defocus are presented in Figures $11-43$ through 11-47; in each case, the enerzy itcident on a given detector at a particular defocused plane was normalized by 
the peak energy incident on the same detector at the best focal plane. By comparing the graphs associated with cilferent detector sizes, it is possible to evaluate the relative merits of using the ge focus detectors in the presence of different aberrations.

It should be pointed out that, because of the nomuniform aperture irradiance function, the energy distribution is a symmetrical about the best focal plane. This asymmetrical property can be mathernatically proven for the simple case in which the input beam has a Gaussian beam profile*, but for the present problem in which the aperture irradiance function, Eq. 11-2, is non-Gaugsian, and the aberration is also nonzero, no analytical solution can be found for the irradiance distribution near the focal region; a numerical technigue must therefors be employed to evaluate the diffraction integrai.

Since the proposed focus sensor detects and compares the energy distribution on both sides of the diifiaction focus, the instrumental error resulting from asymmetrical energy distribution must therefore ie calibrated out by suitably biasing the focus sensor system. The data based on diffraction analysis (e.g. Figures $11-43$ through $11-47$ ) can provide useful indication about the magnitude of the focus correction.

Knife-Edge Trace and Line Spread Function - The knife-edge trace (KET) is a quantitative evaluation of the goint spread fuction; it provides the data for deriving

1. t'se modulation index of a quadrant detector for translation alignment, and

2. the null shift introduced by the presence of asymmetrical components of wavefront aberration (e.g, coma).

The line spread function (LSF) is the derivative of KET. The peak of ISF therefore corresponds to the maximum slope of KET and the maximum modulation index of a quadrant detector. In the presence of coma, Figuses 11-41 and 11-42 show that the lateral displaciment of the peak of LSE

*"Parametric Study of Apertured Focused Gaussian Beams, " D. A. Holmes, J.E. Korka and P. V. Avizonis, Applied Optics, 565, March 1972. 
is different from the amount of null shift as determined from KET; this positional mismatch should be accounted for in optimizing the pointing sensor design.

Dependence of Pointing Sensor Null Shift on Defocusing - If the aberrated input wavefront contains asymmetrical components (such as coma), this will result is null shift errors in the pointing sensor that can vary as the alignment beam is focused. This null shift can be expressed in terms of equivaient energy centroid translation at the serrogate target. The variation of this equivalent translation as a function of defocus error at the target is shown in Figure 11-42. The data used for this plot were derived from KET's at different defosused image planes. In designing the pointing sensor system, Figure 11-42 can provide useful information for budgeting the pointing error tolerances.

Optical Performance Comparison Between Systems $a, b$, and $c$ of the Baseline Configuration - The performance discrepancies resulting from using different target ball sizes were compared: the conclusion was that it was the input aberrations, not the ball sizes, that ultimately jetermined the sensor performance.

Pointing Sensing Based on Nutation Schemes - During the diffraction analysis, preliminary investigation has bein carried out to determine the possible effects of asymmetrical aberration upon the translation pointing error if a nutating alignment beam were employed. Assuming that a pinhole detector was used, the pointing axis would be defined by the effective center point of a selected iso-irradiance contour. Regardless of whether the nutation angle was fixed or variable, there would be a pointing axis error that can vary whenever the alignment beam was defocused. Graphical plots showing these effects are presented in Figure 11-52; these data were derived from through focus point spread functions (PSF). The corresponding input wave aberration was taken to be $1 \lambda$ coma peak to peak.

\section{Computational Results}

Based on the preliminary results generated in the diffraction evaluation of systems $a, b$ and $c$ (see Figure 11-9), system $b$ was selected as the prime 
candidate for detailed analysis; the other two systems were evaluated for the purpose of providing referense performance data. All of the essential computational results have been conveniently reduced to graphical form.

Figures 11-22, 11-23 and 11-25 show the effects of coma upon the PSE. An isometric view of wavefront deformation with $1 \mathrm{k}$ coma is shown in Figure 11-24. Graphs in Figure 11-26 show the variation of peait irradiance on axis versus defocus for different input aberrations; in each case, the graph shows that the diffraction focus is longitudinally shifted from the geometrical focus, where the defocus value is zero. Figure 11-27 shows that, in the presence of asymmetrical aberration ( $1 \lambda$ p-p coma in this case), the true diffraction focus is located by a transverse focal shift in addition to the longitudinal focal shift shown in Figure 11-26. The data presented in Figures 11-22 through Figure 11-27 are all for system a, which has an inherent wavefront aberration of $0.3 \mathrm{~h}$ spherical. This inherent aberration is larger than those for systems $b$ and $c$ becauge system a has much smaller f-number.

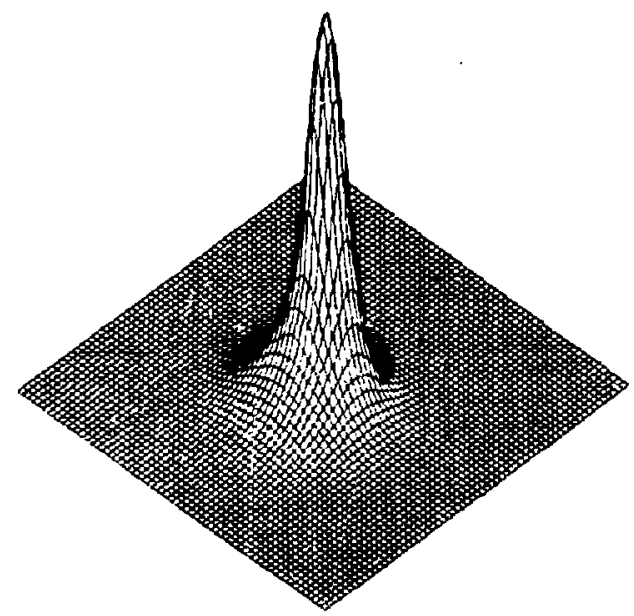

Figure 11-22. Isometric view of point spaced function with $1 \lambda$ coma input wave aber ration. (System (a), $25 \mathrm{~mm}$ ball) 


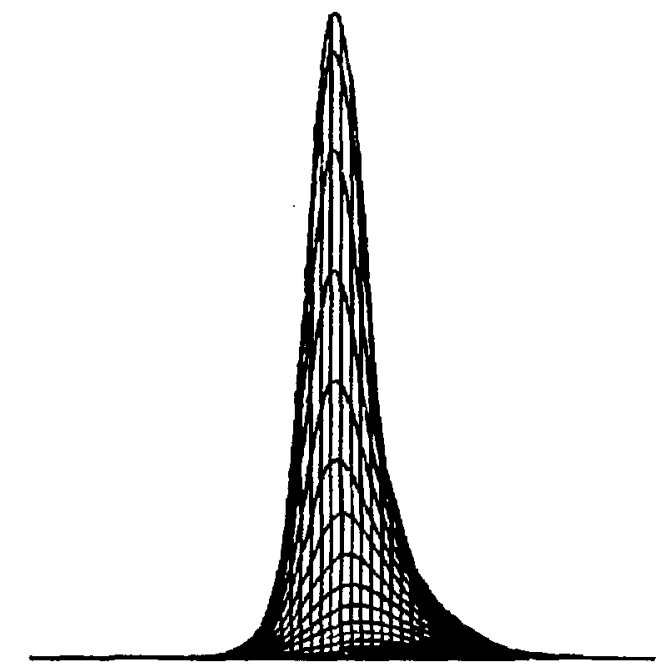

Figure 11-23. Sectional views of point spread function with $1 \lambda$ coma input wave aberration (System (a), $25 \mathrm{~mm}$ ball)

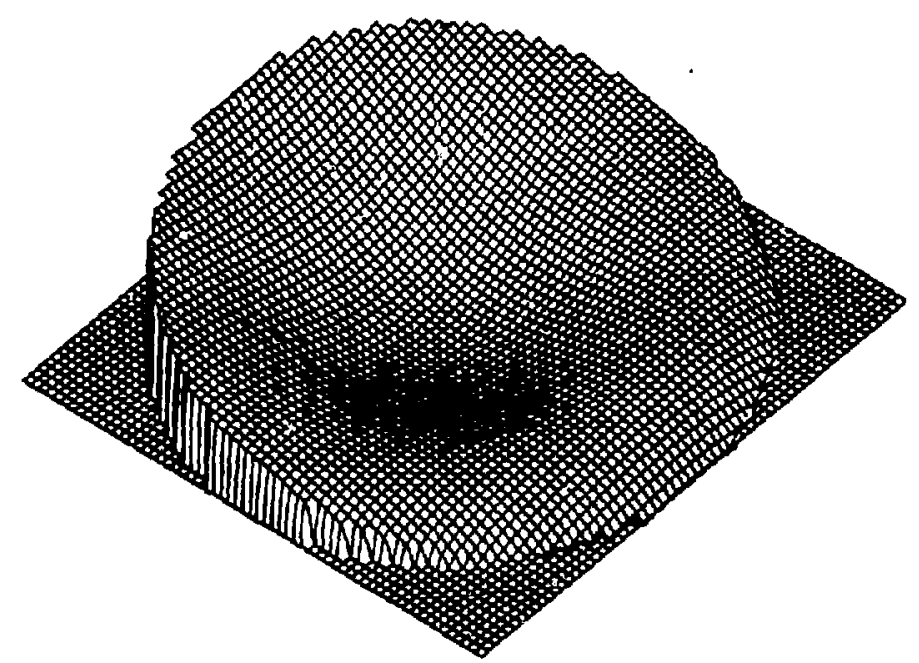

Figure 11-24. Isometric view of wavefront deformation with $1 \lambda$ coma 

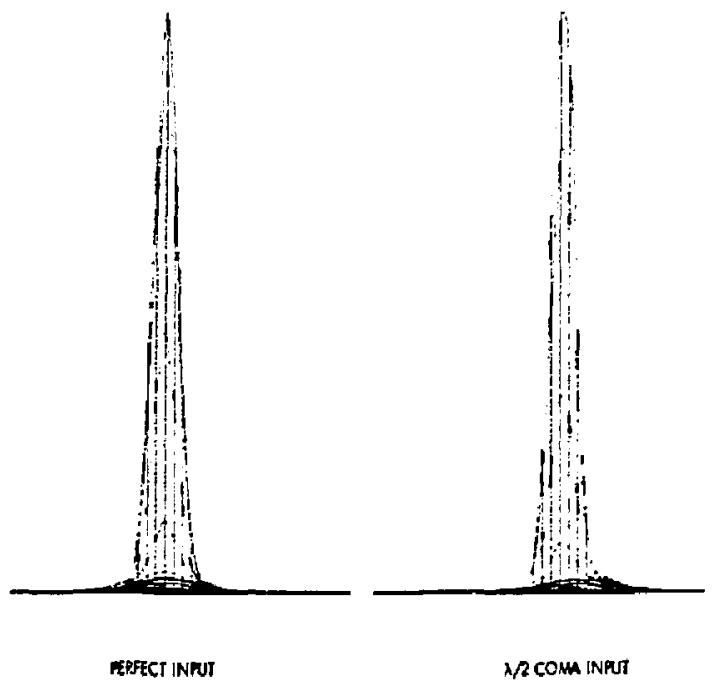

Figure 11-25. Sectional views of point spread functions with and without $\lambda / 2$ coma input wave aberration (System (a), $25 \mathrm{~mm}$ ball)

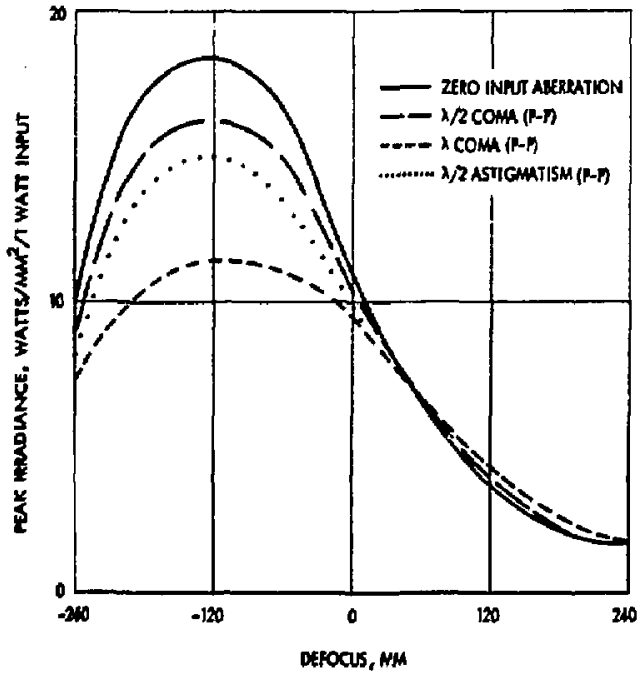

Figure 11-26. Graphs of peak irradiance on axis versus defocus for different input aberrations. (System (a), $25 \mathrm{~mm}$ ball) 


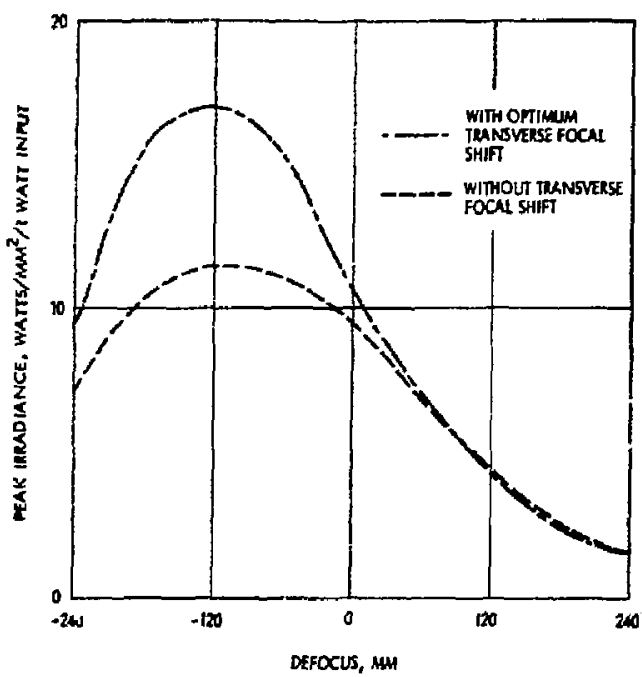

Figure 11-27. A comparison of peak irradiance versus defocus for cases with and without transverse focal shifts. Input wave aberration is $1 \lambda$ coma (p-p). (System (a), $25 \mathrm{~mm}$ ball)

Figure 11-28 gives a comparison of the very small inherent wave aberrations associated with systems $b$ and $c$. Figure 11-29 gives comparison of wave aberration maps of systems $b$ and $c$ in the presence of identical input wavefront aberrations. The wave aberration variances* for these systems are 0.05495 and 0.05251 , respectively. Because of the small numerical differences between these variances, the diffraction performances computed for systems $b$ and $c$ would be very similar; the respective diffraction patterns, irradiance distributions and focal shifts would, of course, be different because their $f$-numbers are not the same.

The computational results of through focus diffraction evaluation for system b will now be presented. Figure 11-30 shows the plots of Y-slice through PSF** at different image planes $1,2,3,4$ and 5 , the input

*The wave aberration variances is expressed in the units of $\lambda^{2}$. **The $Y$-slice through PSF is a sectional view of the PSF in the plane $X=0$. 

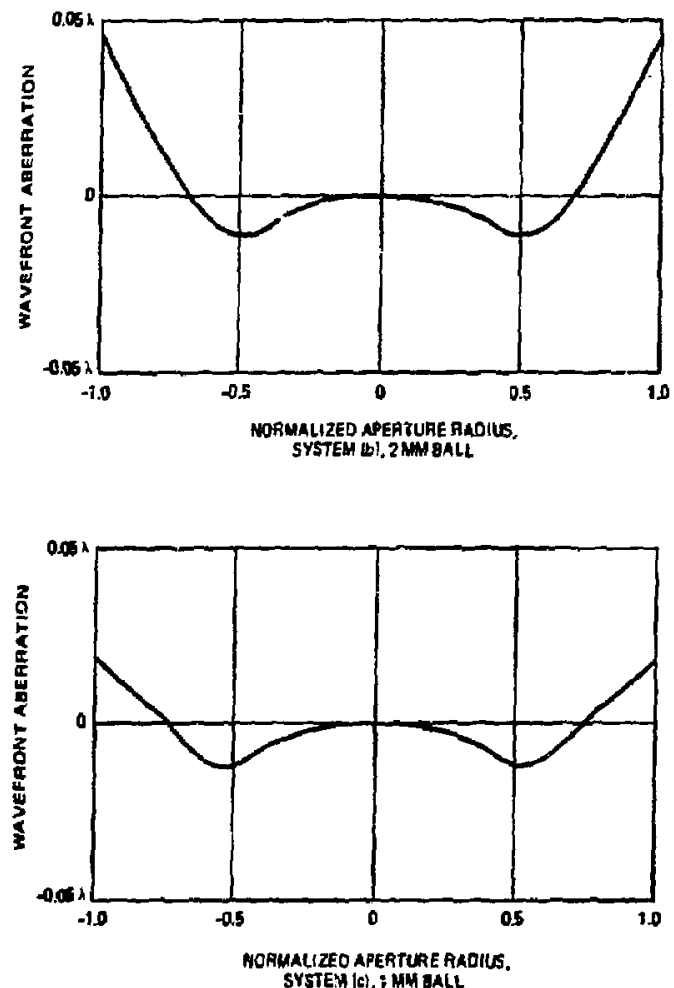

Figure 11-28. A comparison of wave aberration of system (b) and system (c). The input wavefront being perfect 
1

1

I

1

I

I

I

I

I

1

I

I

1

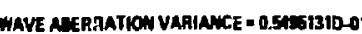

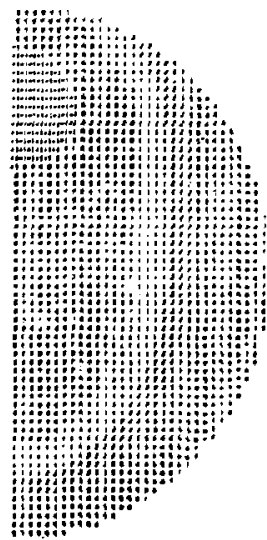

2 MM DAMETER CALL
1 UATT - Q. WAVELEHSTHS

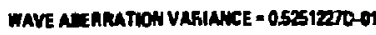

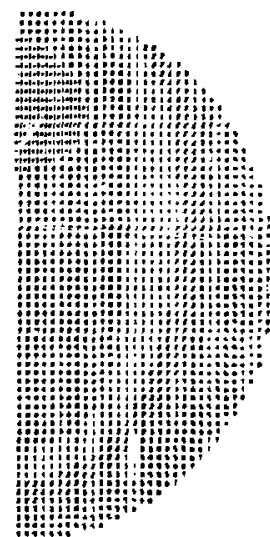

1 KWM DAMETER RAL

Figure 11-29. A comparison of wave aberration maps of sy stem (b) with $2 \mathrm{~mm}$ ball and system (c) with $1 \mathrm{~mm}$ ball, The corresponding input wavefront for each system has $1 / 2 \lambda$ coma, $1 / 2 \lambda$ sphericn $L$ and $\lambda / 2$ astigmatism 


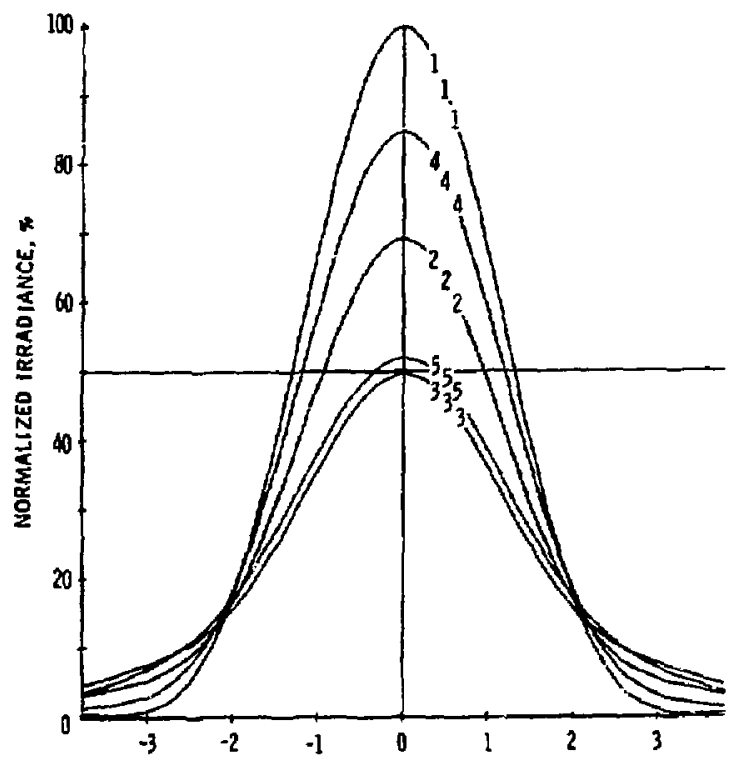

FOCUS AT -5000.0OSYMBOL IS I Y-AXIS, MM

FOCUS AT -18M0.0 SYMBOL IS?

FOCUS AT -25TM.0 SYMBOL IS 3

FOCUS AT 9000.00 SYMBOL IS 4

FOCUS AT 1500.0 SYMBOA IS 5

Figure 11-30. Plots of Y-slice through PSF at different defocused image planes $1,2,3,4$ and 5 . Ac defined above, image plane 1 is defocused $-5000 \mathrm{~mm}$. Input aberration is zero (System (b), $2 \mathrm{~mm}$ ball)

aberration being zerc. The image plane 1 is defocused by -5 meters from the geometrical focus; the other image planes are defocused by different amcunts as defined in the figure. These image space focal shifts can be related to the target space coorlinates in the fol' 'wing way: the nominal f-number of the alignment beam in system $b$ is $f / 2092$, and sincs the focusing lens has an f-number of $\mathrm{f} / 2$, it follows from $\mathrm{Eg}$. (11-1) that one meter defncus at the image plane is approximately equivalent to $1 \mu \mathrm{m}$ logitulinal focal shift at the target; similarly, image plane coordinates (X, Y) (see Eq. 11-5) is geometrically related to the target space coordinates by the tra.1sverse magnification ratio of $10^{3}$. 
Referring again to Figure 11-30, the ordinates define the normalized irradiance with the value of 100 assigned to the peak irradiance correspondir.g to the reference image plane $:$, the irradiance distributions at other image planes are proportionally scaled so that a direct comparison can be made relative to the scaled jrradiance distribution at the reference plane.

Figure 11-31 shows the Y-direction KET plots for the PSFs as represented in Figure 11-30. Each KET is normalized to have a maximum energy of 100 percent. In the absence of any asymmetrical wavefront aberration, the 50 percent energy point must necessarily be on the optical axis, corresponding to zero null shift for a pointing sensor using quadrant detector.

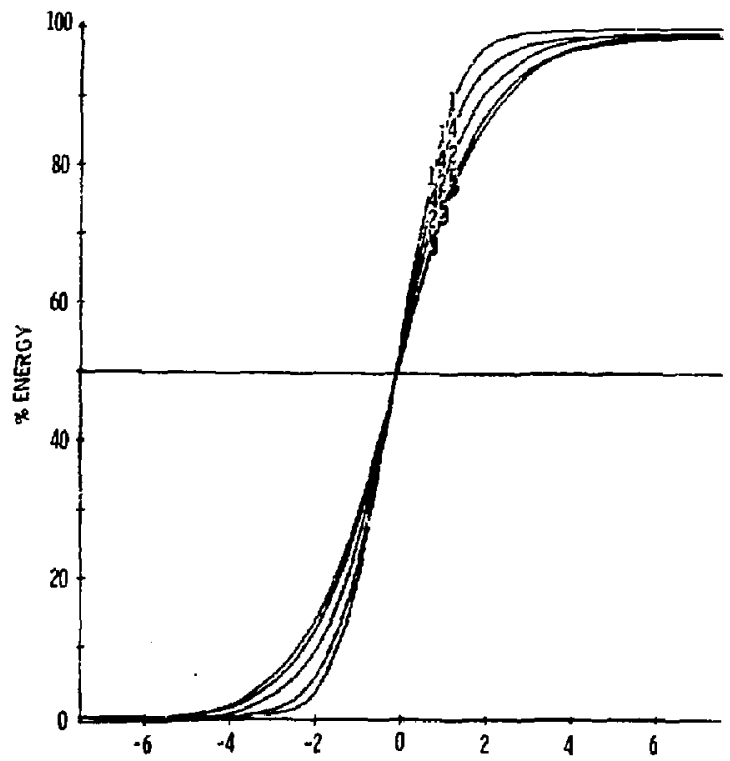

FOCUS AT -5000.00 SYMBOL IS]

Y-AXIS, MM

FOCUS AT -18000 .0 SYMBOL IS ?

FOCUS AT -25000.0 SYMBOL IS 3

FOCUS AT 8000.00 SYMBOL IS A

FOCUS AT 25000.0 SYMBOL IS 5

Figure 11-31. Plots of KET in $Y$-direction at different defocused image planes $1,2,3$, 4 and 5. As defined above, image plane 1 is defocused $-5000 \mathrm{~mm}$. Input aberration is zero. (System (b), $2 \mathrm{~mm}$ ball) 
Diffraction evaluation of syatem $b$ in the presence of different input aberrations will now be considered. Since differeni input aberrations would give rise to different best focal planes, a series of preliminary efforts was made to determine the best focal planes for the different aberrations under consideration. This effort was carried out prior to the selection of the defccused image planes $1,2,3,4$ and 5 .

For the case with input wavefront aber ration given by $1 \lambda$ astigmatism (p-p), the plot of peak irradiance versug defocus is shown in Figure 11-32. Figure 11- 33 shows the plots of Y-slice through PSF at different image planes. Figure 11-3i presents the LSF plats in Y-direction. Again, these plots have been normalized in a similar manne $\mathrm{x}$ as that described above for the PSFs, Figure 11-30. The KET plots in X-and $\mathrm{Y}$-directions for the corresponding series of defocused image planis are given in Figures $11-35$ and 11-36, respectively. The usefulness of these plo's have been discussed above, under prelimithary description of computational results.

For the case with input wayefront aberration represented by $1 \mathrm{~A}$ cona (p-p), plots of X-and Yaslices through PSF at different image planes at a

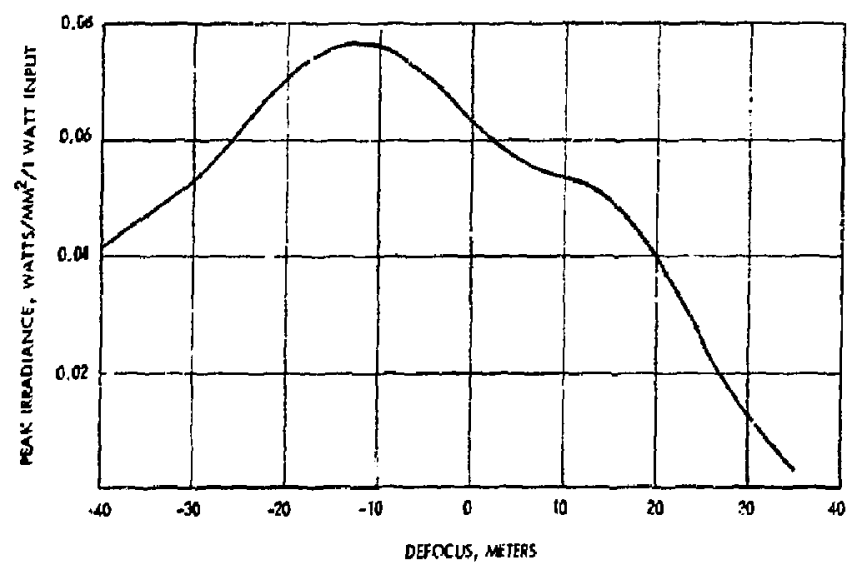

Figure 11-32. Grapi of peak irradiance versus defocus for $1 \lambda$ astigmatism $(p-p)$ input waye aberration. (System (b), $2 \mathrm{~mm}$ ball) 


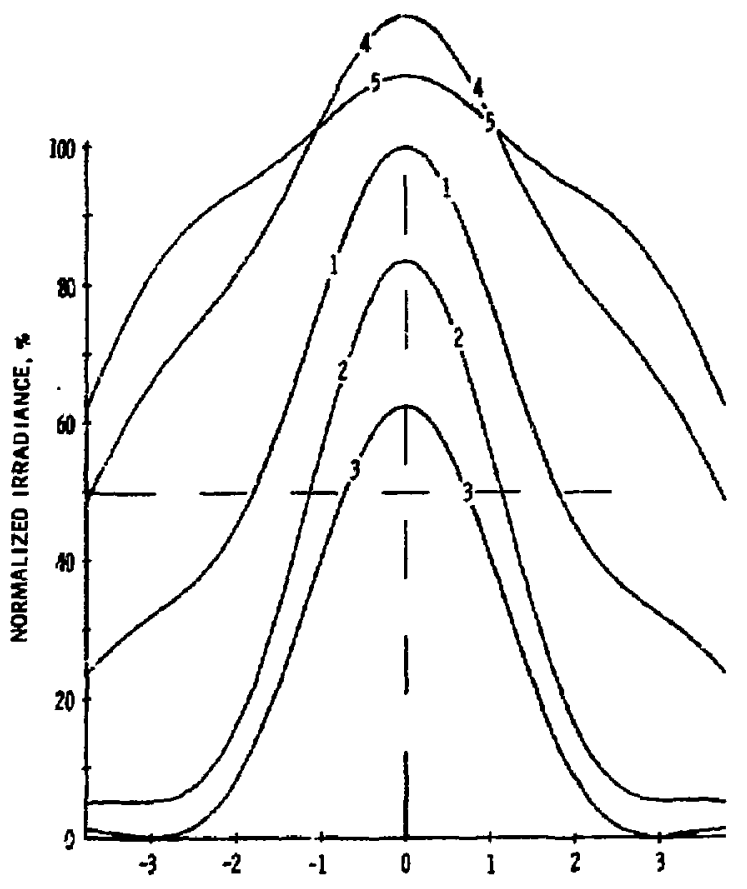

FOCUS AT 0.000000 SYMBOL IS I Y-AXIS, MH FOCUS AT 10000.00 SYMBOC is? FOCUS AT 20000.00 SYABOL IS 3 FOCUS AT -10000.0 SYMBOL IS 4 FOCUS AT -2000,O OSYMBOL IS 5

Figure 11-33. Plots of $\bar{Y}$-slice through PSF at different defecused image planes $1,2,3,4$ and 5 . As defined above, image plane 4 is defocused at $.1000 \mathrm{~mm}$. Input aberration is $1 \lambda$ astigmatism ( $p \cdot p$ ) (System (b), $2 \mathrm{~mm}$ ball). 


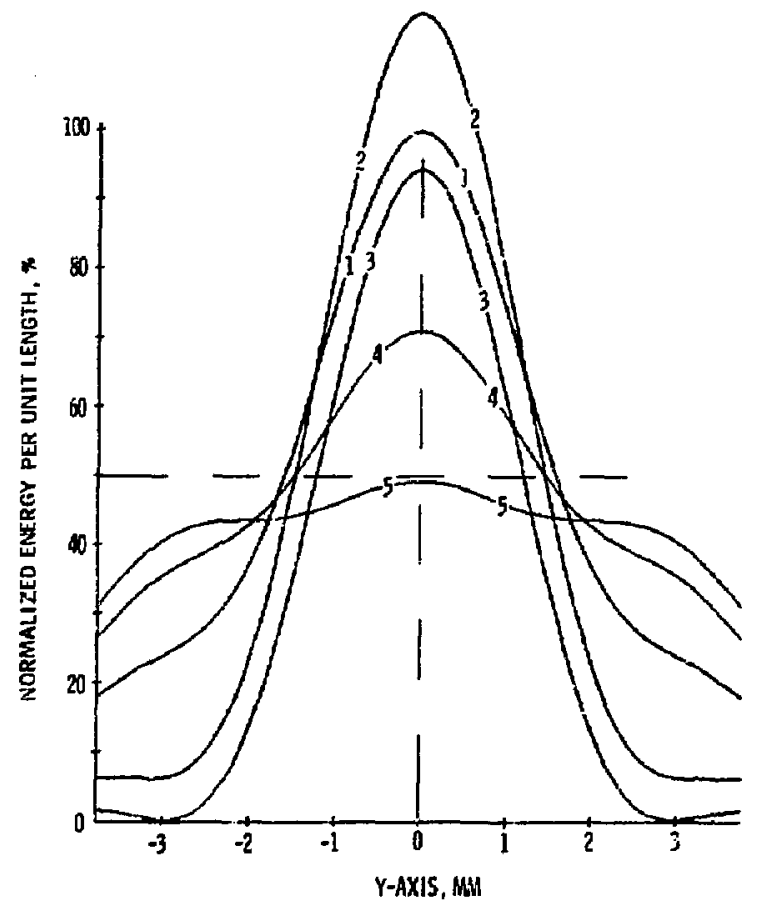

FOCUS AT 0.00000O SYHEO: IS I

FOCUS AT 10000.00 SYMBOL IS?

FOCUS AT 20000.005 YMBOL IS 3

FOCUS AT -10000.0 SYMBOL IS 4

FOCUS AT -20000.0 SYMBOL IS 5

Figure 11-34. Plots of LSF in Y-direction at different defocused image planes $1,2,3$, 4 and 5 . As defined above, image plane 4 is defocused $-1000 \mathrm{~mm}$. Input aberration is $1 \lambda$ astigmatism (p-p) (System (b) $2 \mathrm{~mm}$ ball] 


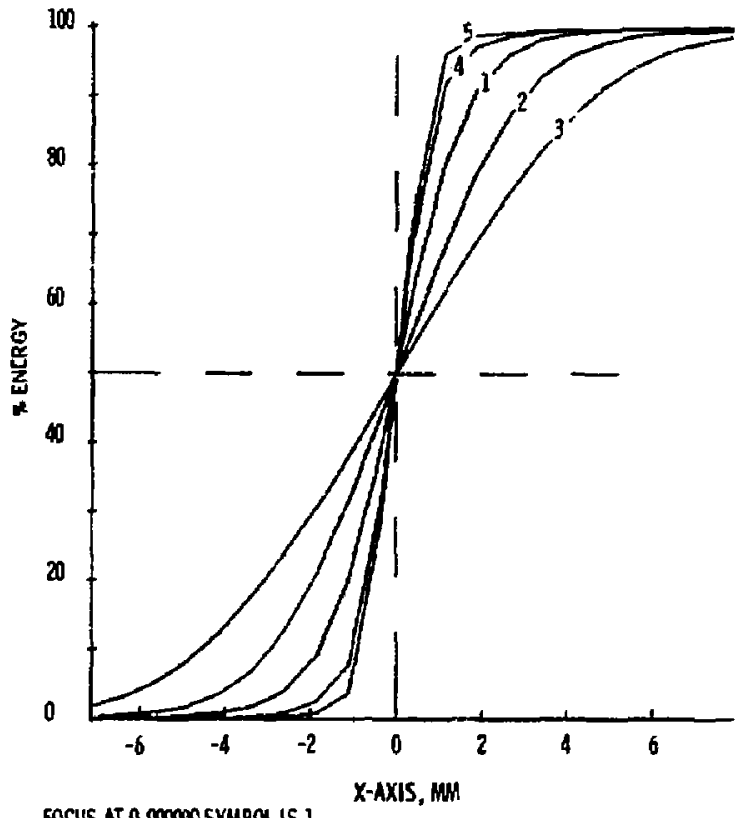

FOCUS AT O. MOOMOSYMBOL IS I

FOCUS AT 10000.00 SYMBOL IS 2

FOCUS AT 20000.00 SYMBOL IS 3

FOCUIS AT -10000.0 SYMBOL IS A

FOCUS AI -20000.0 SYMBOU IS 5

Figure 11-35. Plots of KET in X-direction at different defocused image planes $1,2,3$, 4 and 5. As defined abcve, image plane 4 is defocused $-10000 \mathrm{~mm}$. Input aberration is $I \lambda$ astigmatism (p-p). (System (b), $2 \mathrm{~mm}$ ball) 


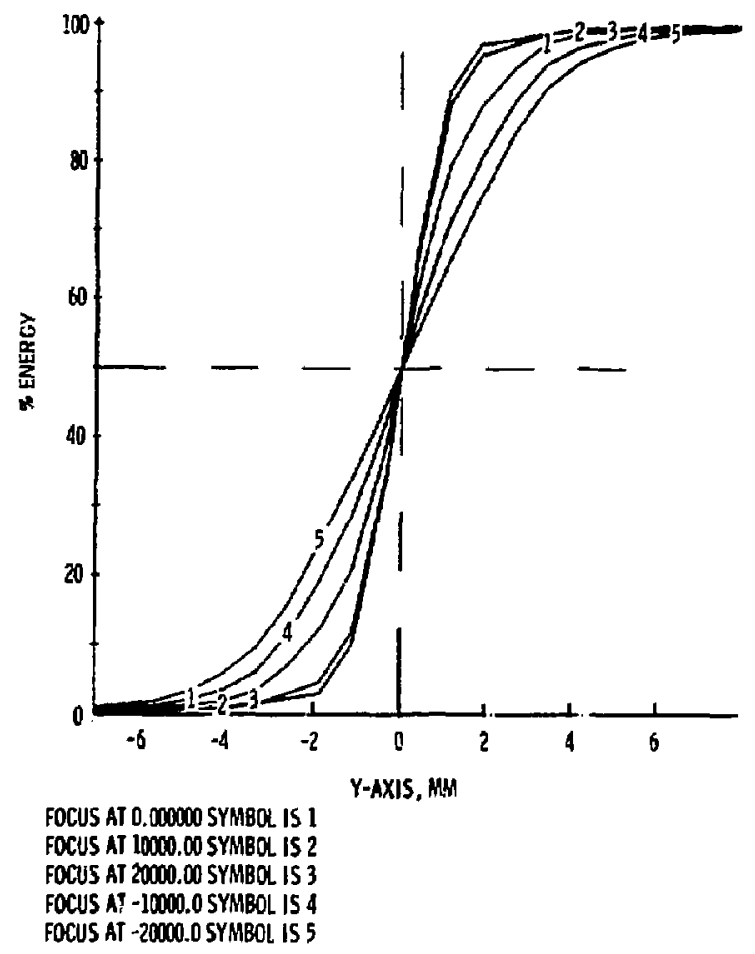

Figure 11-36. Plots of KET in Y-direction at different defocused image planes $1,2,3$, 4 and 5. As defined above, image plane 4 is defocused $-10000 \mathrm{~mm}$. Input aberration is $1 \lambda$ astigmatism (p-p). (System (b), $2 \mathrm{~mm}$ ball)

given in Figures 11-37 and 11-38, respectively. Figures $11-39$ and $11-40$ show the graphs of LSF in X-and $Y$-directions at different defocused image planes. Figure 11-41 gives the KET plots in Y-direction, As pointed out above a careful comparison between Figures $11-40$ and $11-41$ show that, for corresponding image planes, the lateral displacements of the peak of LSF are different from the amounts of null shift as determined from KET, This implies that the presence of $1 \lambda$ coma ( $p-p)$ results in the maximum slope of the KET not occurring at the 50 percent energy point; this effect should be accounted for in the optimization of the pointing sensor design. 


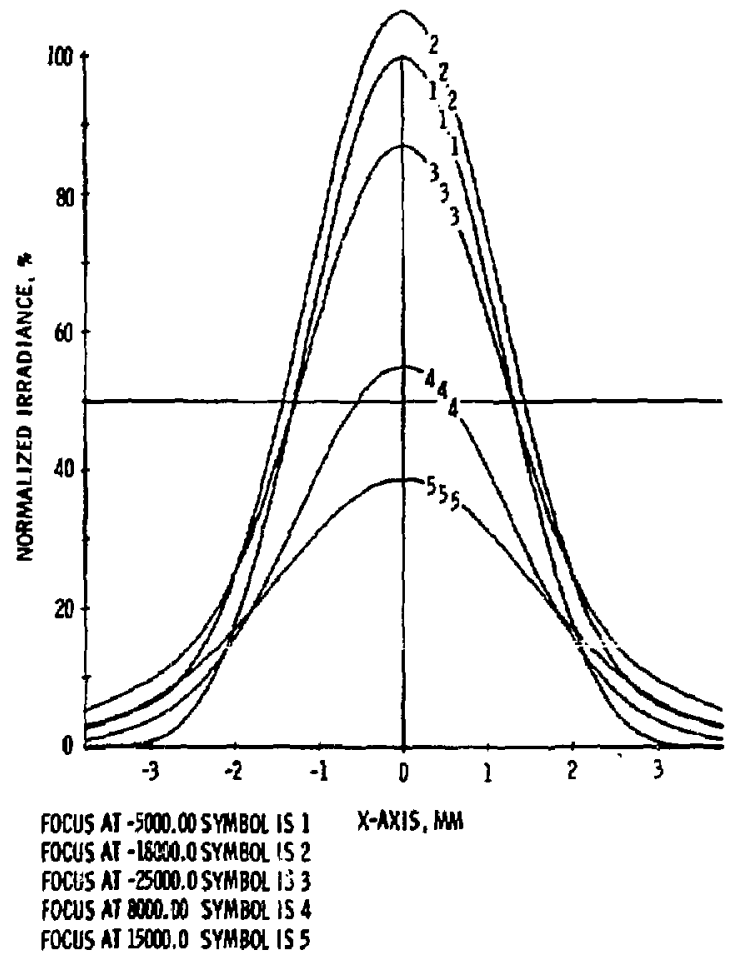

Figure 11-37. Plots of X-slice through PSF at different defocused image planes $1,2,3,4$ and 5. As defined above, image plane 1 is deiocused $-5000 \mathrm{~mm}$. Input aberration is ith coma p-p. (System (b). $2 \mathrm{~mm}$ ball) 


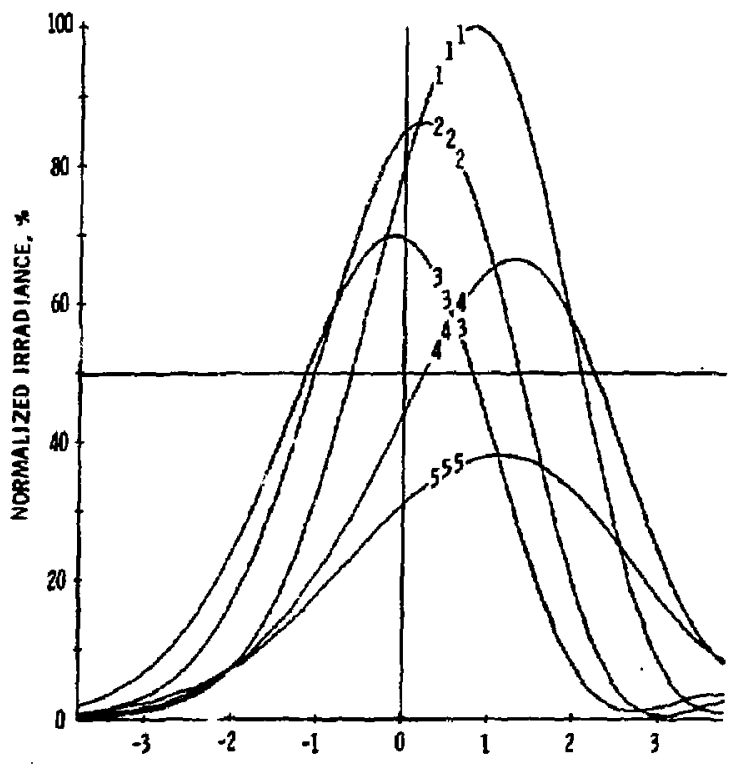

FOCUS AT -5000.00 SYMBOL IS I Y-AXIS, MH

FOCUS AT -18000. OSYMBOL IS 2

FOCUS AT - 25000.0 SYMBOL IS 3

FOCUS AT 2000.00 SYMBOC is 4

FOCUS AT I5OOD.0 SYMBOL IS 5

Figure 11-38, Plots of Y-slice through PSF at different defocused image planes $1,2,3,4$ and 5 . As defined above, image plane 1 is defocused $-5000 \mathrm{~mm}$. Input aberration is $1 \lambda$ coma p-p. (System (b), $2 \mathrm{~mm}$ ball). 


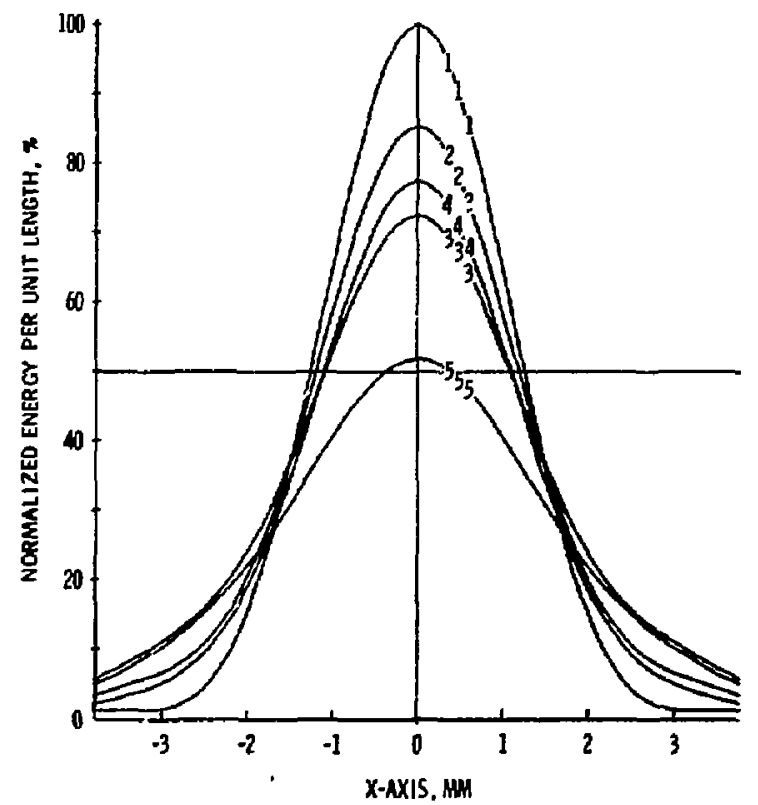

FOCUS AT -5000,DOS SYMBOL IS I

FOCUS AT +18000.0SYMBOL 152

FOCUS AT -2500.0 DSYMBOL IS 3

FOCUS AT 8000.00 SYMBOL IS 4

FOCUS AT 15000.0 SYMEOL 155

Figure 11-39. Plots of LSF in X-direction at different defocused image planes $1,2,3$, 4 and 5. As defined above, image plane 1 is defocused $-5000 \mathrm{~mm}$, Input aberration is li coma (p-p) (System (b), $2 \mathrm{~mm}$ ball) 


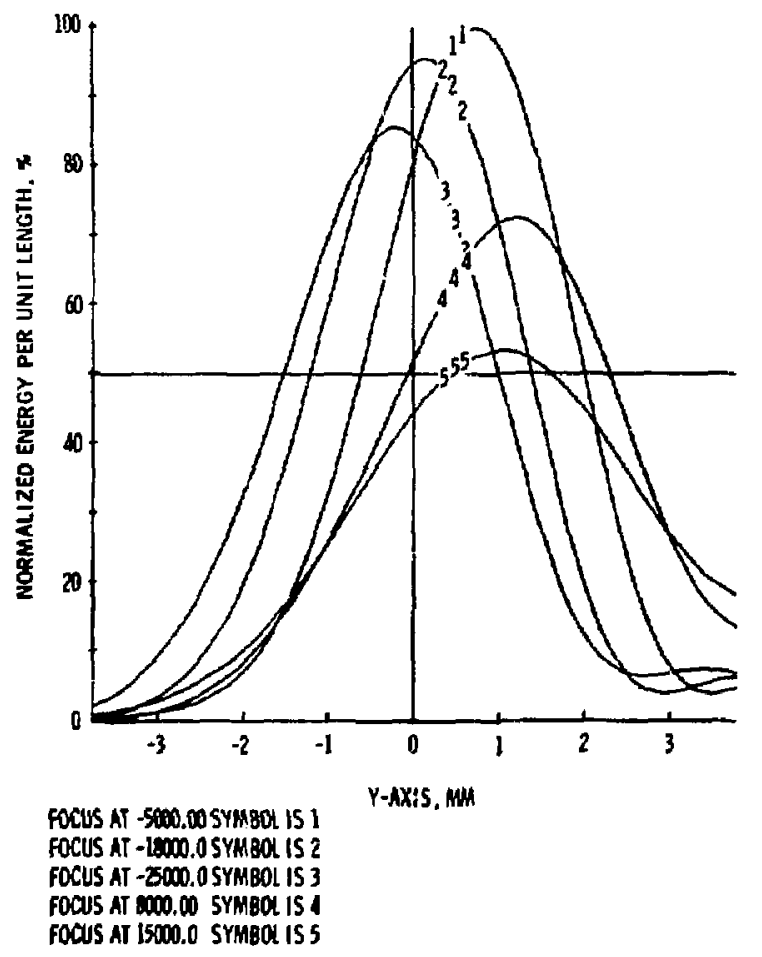

Figure 11-40. Plots of LSF in Y-direction at different defocused image planes $1,2,3$, 4 and 5. As defined above, image plane 1 is defocused $-5000 \mathrm{~mm}$. Input aberration is $1 \lambda$ coma (p-p) (System (b), $2 \mathrm{~mm}$ ball) 


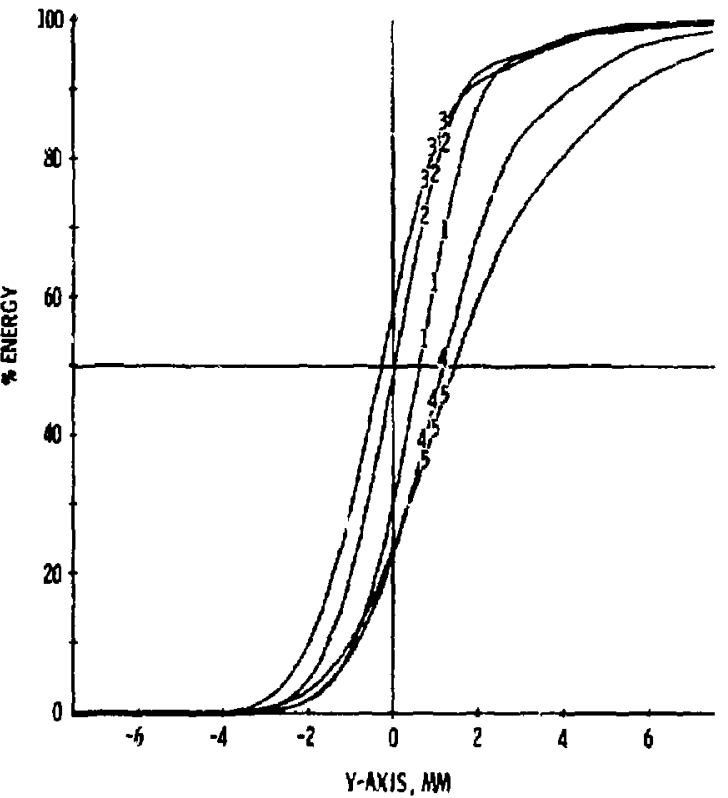

FOCUS AT -5000.00 SYMBOL IS 1

FOCUS AT -10000.0 SYMBOOL IS 2

FOCUS AT -25500.0 SYMBOQ IS 3

FOCUS AT SOO.00 SYMBOL IS 4

FOCUS AT ISOO0.0 SYMBOL IS 5

Figure 11-4l. KET plots in Y-direction at different defocused image planes $1,2,3$, 4 and 5. As defined above, image plane 1 is defocused $-5000 \mathrm{~mm}$. Input aberration is $1 \lambda$ coma $p-p$ (System (b), $2 \mathrm{~mm}$ ball) 


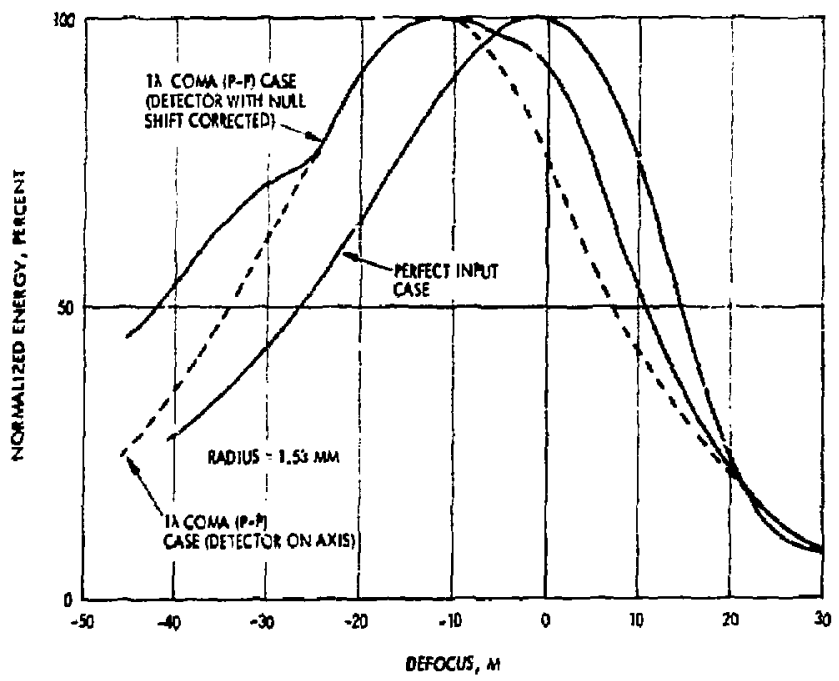

Figure 11-44. Graphs of normalized energy incident on detector versus defocus for the perfect input case and the 11 corna ( $\mathrm{p}-\mathrm{p}$ ) case with detector located (1) at position with null shift corrected, and (2) on axis. Radius of detector is $1.53 \mathrm{~mm}$. Before normalization, this defector size encompasseg, at the best focus, 52 percent of the input energy for the perfect case and 46 percent of the input energy for the $1 \lambda$ coma case. (System (b), $2 \mathrm{~mm}$ ball) 


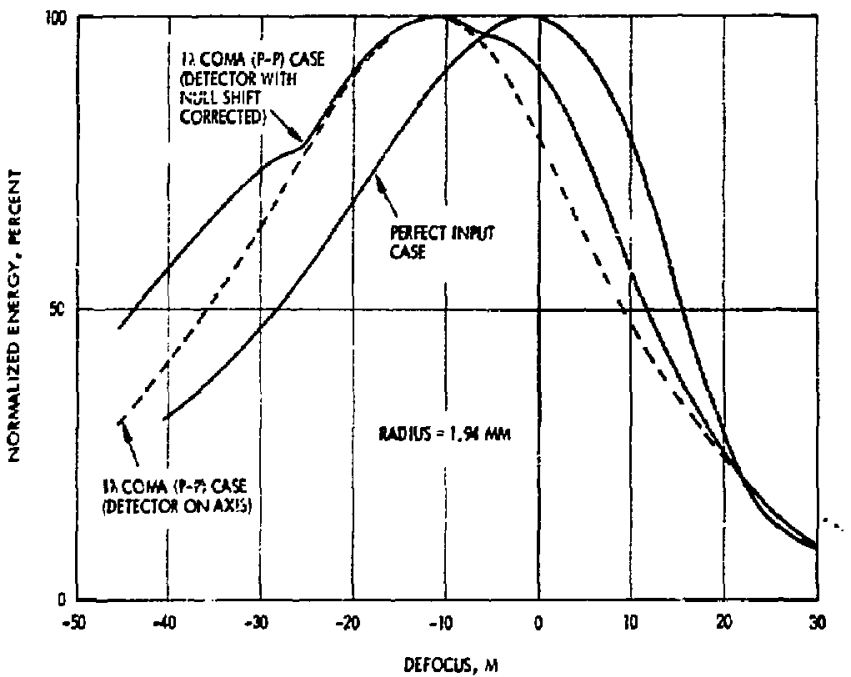

Figure 11-45. Graphs of normalized energy incident on detector versus defocus for the perfect input case and the 17 coma (p-p) case with detector located (1) at position with null shift corrected, and (2) on axis. Radius of detector is $1.94 \mathrm{~mm}$. Before normalization, this de tector size encompasses, at the best focus, 76 percent of the input energy for the perfect case and 69 percent of the input energy for the li coma case. (Systen (b), $2 \mathrm{~nm}$ ball) 


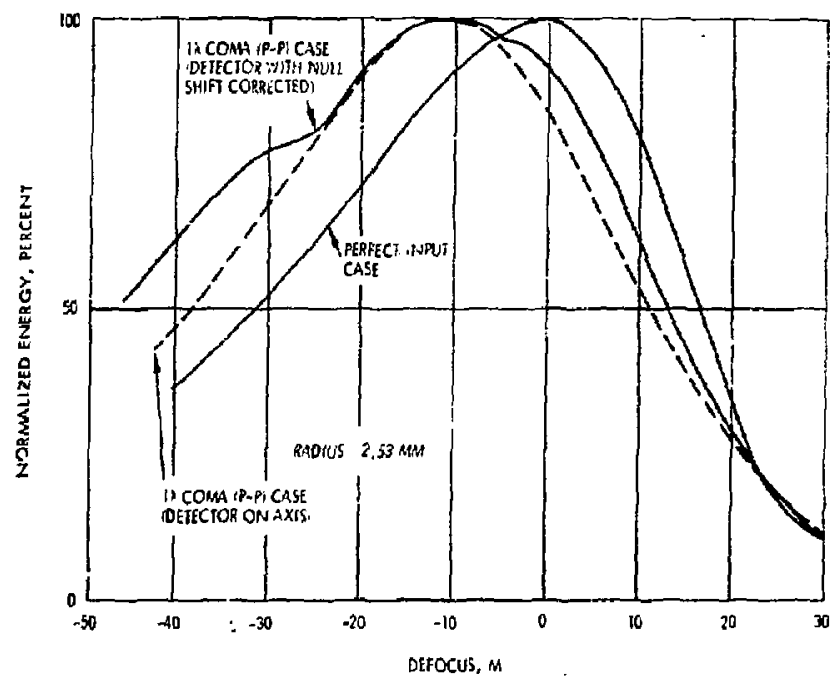

Figure 11-46. Graphs of normalized energy incident on detector versus defocus for the perfect input case and the $1 \lambda$ coma ( $p$-p) case with detector located ( 1 ) at position with null shift corrected, and (2) on axis. Radius of detector is $2.53 \mathrm{~mm}$. Before normalization, this detector size encompasses, at the best focus, 85 percent of the input energy for the perfect case and 79 percent of the input energy for the $1 \lambda$ coma case (System (b), $2 \mathrm{~mm}$ ball) 
14 percent of the total input energy at the diffraction focus ; this encompassed energy, together with other encompassed energies at different defocused detector positions, were all scaled up by 100/14; the results were then plotted in Figure 11-43 as the noima zed colid curve for the perfect input case. In the presence of $1 \mathrm{~A}$ coma $(p-p)$, the same detector size was found to encompass only 12 persent of the total input energy at the diffraction focus ${ }^{\text {th }}$, the normalized solid curve in Figure 11-43 for 1: coma case wae then derived in a similar way as that for the perfect input case, except that the energr normalization was achieved by using a scaling factor of $10 \mathrm{c} / 12$. The no:malized broken curve in Figure 11-43 for $1 \lambda$ coma case shows the normalized results corresponding to the same detector but sampling the incident energy along the optical axis (i.e. the pointing error resulting from coma was not corrected out prior to the energy sampling by the focus detector).

Figure 11-47 whows the normalized energies contained within forr focus detector radii $(0.51 \mathrm{~mm}, 1.55 \mathrm{~mm}, 1.97 \mathrm{~mm} \text { and } 2.53 \mathrm{~mm})^{\text {t.t. }}$ as a function of defocus; the data used here are extracted from Figures 11-43 through 11-46 for the case corresponding to $1 \lambda$ coma ( $p-p)$ input wave aberration and with the pointing errors corrected out. Referring to Figure 11-47, all the four curves lie reasonably close to each other, signifying that all four focus detector sizes have similar energy senaing capabilities, a ssuming that the signal/noise is not a limiting factor, and that the input energy can be artitrarily increased to compensate for the seduced-size detectors.

It has been pointed out earlier that, due to the parabolic aperture apodization and the presence of aberration, the normalized energy distribution as a function of defocus is asymmetrical about the diffraction focus fsee Figure (1-47). Since the proposed focus sensor detects and compares the

\footnotetext{
* The energy encompassed within a selected radius at a given image plane was determined from the appropriate radial energy distribution (RED) date - wee Eq. 11-7. The RED is dependent on the input aberration and the amount of defocus of the image plane.

** A seen from Figure 11-38, the presence of in coma in thi input beam results in a diffraction focus which hae both nonzero longitidinal and transver sai focal shifts from the geometrical focus.

${ }^{*}$ These values of detector radius encompass 14, 52, 69 and 85 percent of energy of perfect input case.
} 


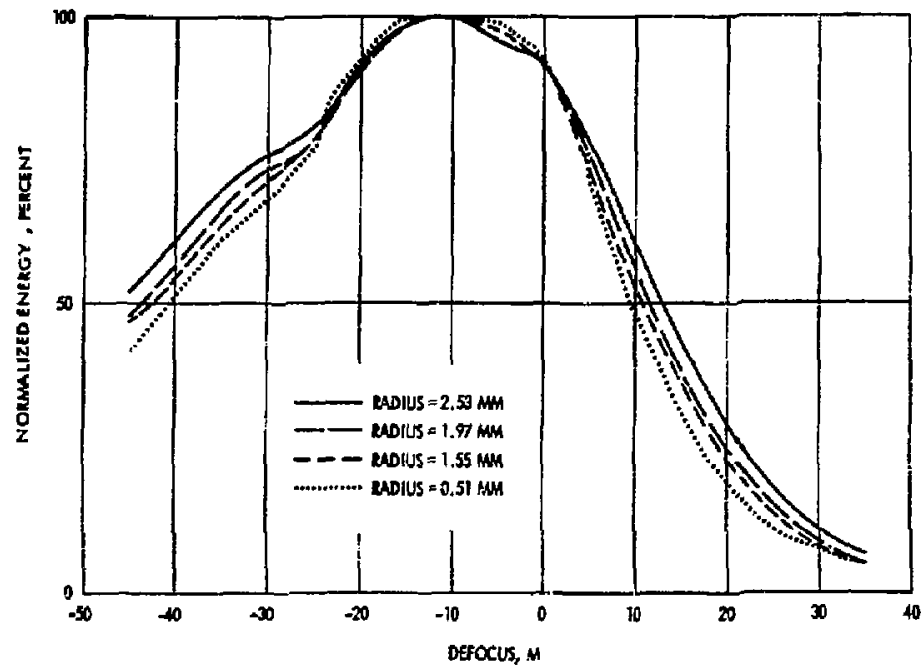

Figure 11-47, Graphs of normalized energies contained within four focus detector radi $(0.51 \mathrm{~mm}, 1.55 \mathrm{~mm}, 1.97 \mathrm{~mm}$ and $2.53 \mathrm{~mm})$ as a function of defocus. Date used here are extracted from Figures $11-43$ through $11-46$ for the case corresponding to $1 \lambda$ coma (p-p) input wave aberration and with the nuli shift corrected out. (Gystem (b), $2 \mathrm{~mm}$ ball)

energy distribution on both sides of the diffraction focus, the instrumental error resulting from asymmetrical energy distribution must the refore be calibrated out by suitably biasing the focus sensor system.

The final portion of the diffraction evaluation was carried out for the case with input wavefront abberration given by $\frac{1}{2} \lambda$ coma ( $\left.p-p\right), \frac{1}{2} \lambda$ a stigmatism (p-p) and $\frac{1}{2} \lambda$ spherical. Figure $11-48$ shows the plots of LSF in $Y$-direction at different defocused image planes; graphs of KET in X-and $\mathrm{Y}$-directions corresponding to the se image planes are given in Figures $11-49$ and 11-50. Based on direct comparisons between the se results and those presented earlier, it is clear that the asymmetrical wavefront aberrations (such as comal can cauge greatest degradation in sensor performance. These aberrations not only introduce added problems in optimizing the modulation index of sensor, but can also cause null shifts in the pointing sensor that vary with defocus. 


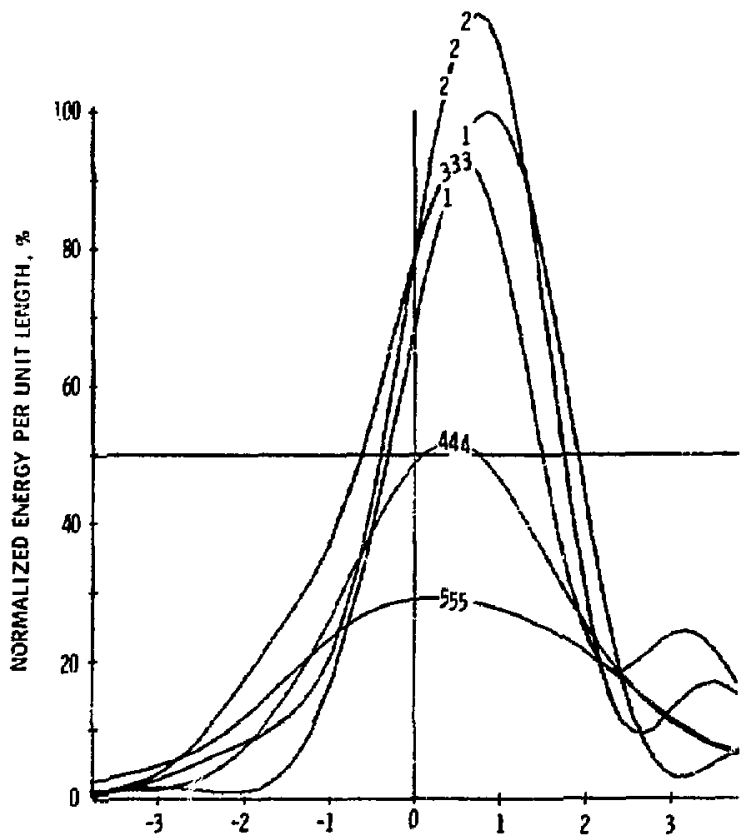

FOCUS AT -5000 .00 SYMBOL IS I Y-AXIS, MM

FOCLS AT - 18000 . OSYMBOL IS 2

FOCUS AT - 25F00.0 SYMBOL IS 3

FOCUS AT 2000.00 SYMBOL IS 4

FOCUS AT 15000.0 SYMBOL IS 5

Figure 11-48. Plots of LSF in Y-direction at different defocused image planes $1,2,3,4$ and 5 . As defined above, image plane $I$ is defocused $-5000 \mathrm{~mm}$. Input wavefront aber ration include $3 / 2 \lambda$ coma (p-p), $1 / 2 \lambda$ astigmatiom ( $p-p)$ and $1 / 2 \lambda$ spheriral. (System (b), $2 \mathrm{~mm}$ ball) 


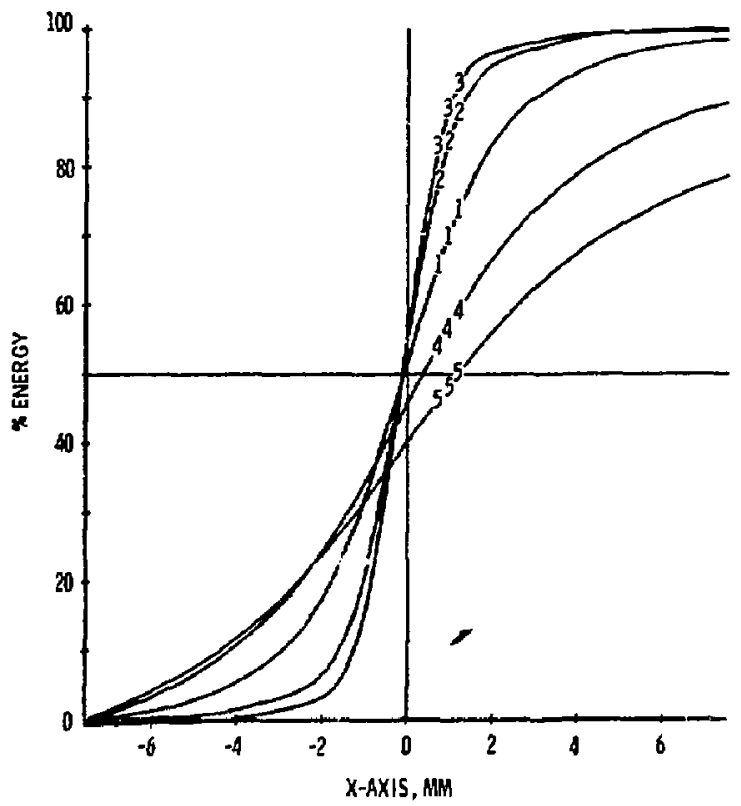

FOCUS AT -5A00.0O SYMBBOL IS I

FOCUS AT - $16000.05 \mathrm{YMBOL}$ IS 2

FOCUS AT -25000.0 SYMBOL IS 3

FOCUS AT 8000.00 SYMBOL IS A

FOCUS AT 15000.0 SYMBCL IS 5

Figure 11-49. Plots of KET in X-direction at different defocused image planes $1,2,3,4$ and 5 . As defined above, image plane 1 is defocused $-5000 \mathrm{~mm}$. Input wayefront aberration includes $\lambda / 2$ coma $(p-p), 1 / 2$ astigmatism ( $p-p)$ and $\lambda / 2$ spherical. (System (b), $2 \mathrm{~mm}$ ball) 


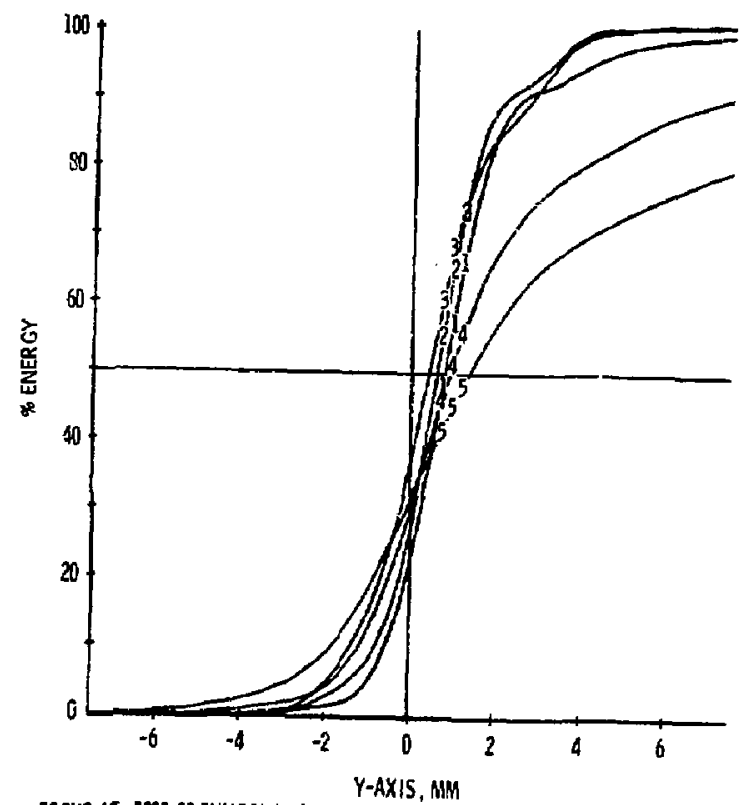

FOCUS AT -5000.00 SYMBOL IS I

FPCUS AT -18000.0 SYMBOL 152

FOCLS AT-25000.0 SYMBOL 153

FOCUS Át 8000.00 SYMB BOL is 4

FOCUS AT BSWO. SYMBOL IS 5

Figure 11-50. Plots of KET in Y-direction at different defocused image planes $1,2,3,4$ and 5 . As defined above, image plane $i$ is defocused $-5000 \mathrm{~mm}$. Input wavefront aberration inc ludes $1 / 2 \lambda$ coma $(p-p), 1 / 2 \lambda$ astigmatism $(p-p)$, and $\lambda / 2$ spherical. (System $(b)$, $2 \mathrm{~mm}$ ball)

\section{Pointing sensing based on nutation schemes}

During the diffractior analysis, preliminary investigation has been carried out to determine the possible effects of asymmetrical aberration upon the translat:ia pointing error if a nutating alignment beam were employed. Assuming that a pinbole detector was used, the pointing axis would be defined by the effective center point of a selected iso-irradiance contour of the diffraction blur. Regarcless of whether the nutation angle was fixed or variable, there would be a pointing axis error that can vary whenever the alignment beam was defocused. Figure 11-51 shows the iso-irradiance conlour plots 

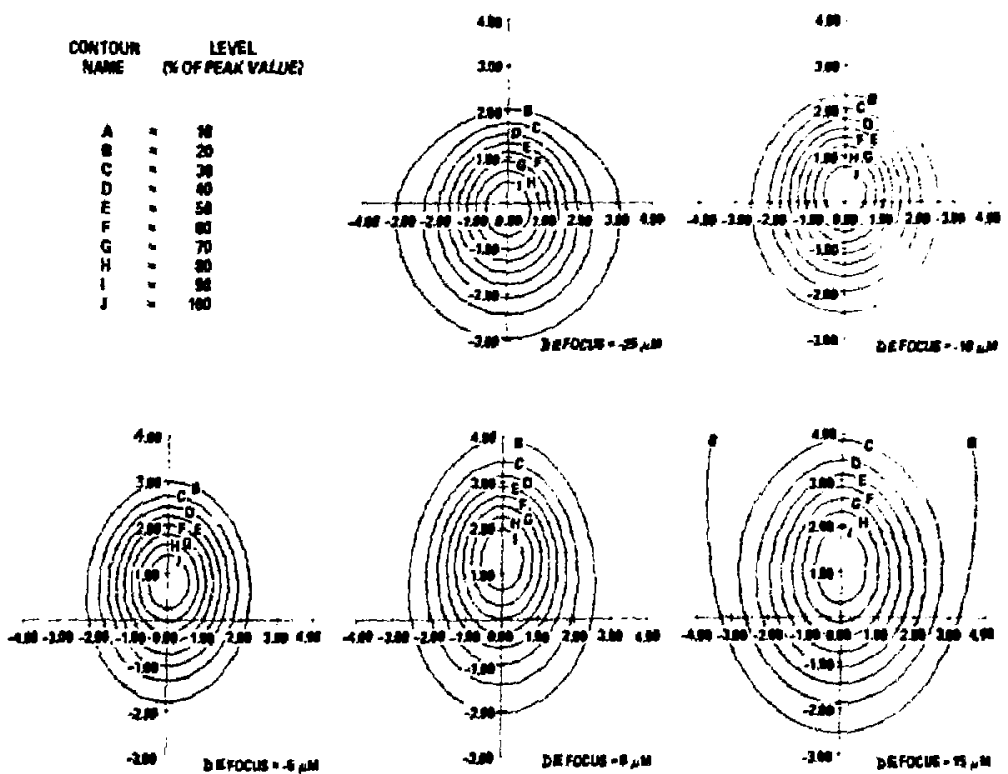

Figure 11-51. Through focus iso-irradiance contour plots of PSF. Input wave-aberration is $l \lambda$ coma $(p-p)$

of the PSF at different focal planes, the input wave-aberration being $1 \lambda$ coma (p-p); this figure was derived from the PSF shown in Figure 11-38. For convenience, both the graphical scales and the defoens values in Figure 11-51 are expressed in terms of tayget space coordinates. By using Figure 11-51, it is pos sible to estimate the variation of the pointing error with defocus. Graphs in Figure 11-52 show the transverse null shift versus defocus for two different nutation angles: the upper curve corresponds to the case with equivalent radius of nutation at target of $1.83 \mu \mathrm{m}$, which is half of the distance between the inter section points of the 75 percent iso-irradiance contour with the axis of ordinates in the best focal plane (see contour plats at defocus $=-5 \mu \mathrm{m}$ in Figure $11-51$ ); the lower curve correspond 8 to the case with equivalent radius of nutation of $2.77 \mu \mathrm{m}$, which is as sociated with the 50 pereent iso-irradiance contour. 


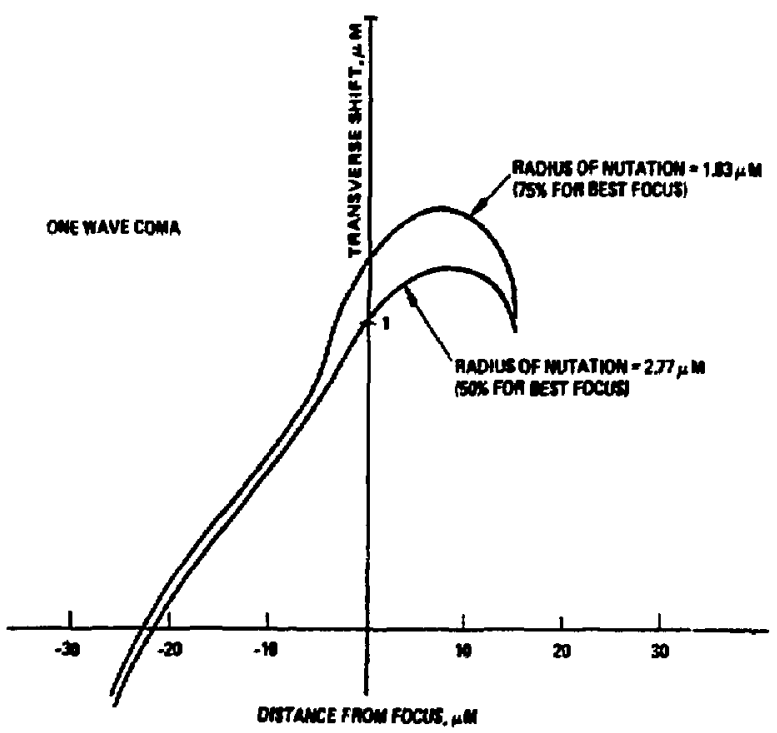

Figure 11-52. Graphs of transverse null shift versus defocus for two nutating input beams. The input wave-aber ration is IX coma (p-p)

\section{Sensor Package Sensitivity Study}

One of the factors influencing the choice of a sensor design configuration and its parameters is the sersitivity of the sensor system to: 1) input beam steering, 2) axial and lateral movements of the surrogate target, (or equivaleritly the focusing lens), and finally, 3) angular and translational displacements of the sensor package itself. Throughout this section, all angular quantities will be denoted by $\Delta \theta_{i}{ }^{\prime} s$ whereas lateral displacements will be denoted by $\Delta Y_{i}^{\prime}$ 's and axial movements by $s Z_{i}{ }^{\prime} s$. The subscript $i$ can be one of the following: The input beam, (I), the focusing lens, (L), the sensor lens, ( $($ ), the focus sensor detector, (D), and finally the beam centering detector\%, (CD).

Since the purpose of the sensor package is for the alignment of the input beam, the focusing lens and the surrogate target, its sensitivity to their positioning should be maximizer,. On the other hand, 28 has been mentioned

HAs described in an appendix of this report, an alternative centering sensor scheme is used in the finalized alignment configuration. 
at the beginning of this Section, it should be reasonably insensitive to its own positioning relative to the surrogate target so that the system instrumental errors can be minimized. The criterion for the system sensitivity to angular and lateral displacements is chosen to be the relative motion of the 50 percent energy point of the focused spot on the sensor detector. For the axial displacements, the criterion is the growth of the spot diameter on the detector.

As was the case in the parametric trade-off study above, the sensitivity investigation was carried out in several stages. First, with collimated input beam and the $\mathrm{f} / 2$ focusing lens, the effects of $\Delta \theta_{I}, \Delta \mathrm{Y}_{\mathrm{L}}, \Delta \mathrm{Y}_{\mathrm{l}}+\mathrm{D}^{\prime} \Delta \mathrm{Z}_{\mathrm{L}}$ and $\Delta Z_{l+D}$ were examined. The results are listed in Tables $11-5$ through $11-9$.

Table 11-5. Sensitivity of sensor package to collimated input beam steering; with $\mathrm{f} / 2$ focusing lens and $1 \mathrm{~mm}$ diameter ball

\begin{tabular}{|c|c|}
\hline$\Delta \theta_{1}$ of Input Beam & $\begin{array}{c}\text { Lateral shift of } 50 \text { percent energy point } \\
\text { of fochsed spot on detector }\end{array}$ \\
\hline $0 \mu \mathrm{rad}$ & $0.000 \mathrm{~mm}$ \\
$1 \mu \mathrm{rad}$ & $0.059 \mathrm{~mm}$ \\
$2 \mu \mathrm{rad}$ & $0.118 \mathrm{~mm}$ \\
$4 \mu \mathrm{rad}$ & $0.235 \mathrm{~mm}$ \\
\hline
\end{tabular}

Table 11-6. Sensitivity of sensor package to lateral dis placements of the $\mathrm{F} / 2$ focusing lens; with collimated input beam and $1 \mathrm{~mm}$ diameter ball

\begin{tabular}{|c|c|}
\hline$\Delta Y_{\text {L or } B}$ & $\begin{array}{c}\text { Lateral shift of } 50 \text { percent energy point } \\
\text { of focused spot on detector }\end{array}$ \\
\hline $0.0000 \mathrm{~mm}$ & $0.000 \mathrm{~mm}$ \\
$0.0005 \mathrm{~mm}$ & $0.030 \mathrm{~mm}$ \\
$0.0010 \mathrm{~mm}$ & $0.059 \mathrm{~nm}$ \\
$0.0020 \mathrm{~mm}$ & $0.118 \mathrm{~mm}$ \\
\hline
\end{tabular}


Table 11-7. Sensitivity of sensor package to its own lateral displacements; with collimated input beam, $\mathrm{f} / 2$ focusing lens and $1 \mathrm{~mm}$ diameter ball

\begin{tabular}{|c|c|}
\hline$\Delta Y_{\text {}}+\mathrm{D}$ & $\begin{array}{c}\text { Lateral shift of } 50 \text { percent energy point } \\
\text { of focused spot on detector }\end{array}$ \\
\hline $0.0 \mathrm{~mm}$ & $0.000 \mathrm{~mm}$ \\
$0.5 \mathrm{~mm}$ & $0.012 \mathrm{~mm}$ \\
$1.0 \mathrm{~mm}$ & $0.024 \mathrm{~mm}$ \\
$2.0 \mathrm{~mm}$ & $0.048 \mathrm{~mm}$ \\
\hline
\end{tabular}

Table 11-8. Sersitivity of sers or package to axial movements of the $\$ / 2$ focusing lens; with collimated input beam and $1 \mathrm{~mm}$ diameter bali

\begin{tabular}{|l|c|}
\hline$\Delta Z_{\text {L or B }}$ & $\begin{array}{c}\text { Spot diameter } \\
\text { on detector }\end{array}$ \\
\hline $0.000 \mathrm{~mm}$ & $0.253 \mathrm{~mm}$ \\
$0.001 \mathrm{~mm}$ & $0.278 \mathrm{~mm}$ \\
$0.005 \mathrm{~mm}$ & $0.379 \mathrm{~mm}$ \\
$0.010 \mathrm{~mm}$ & $0.505 \mathrm{~mm}$ \\
\hline
\end{tabular}



Table 11-9. Sensitivity of sensor package to its own axial movements; with collimated input beam, $\mathrm{f} / 2$ focusing lens and $l \mathrm{~mm}$ diameter ball

\begin{tabular}{|c|c|}
\hline$\Delta \mathrm{Z}_{\ell+\mathrm{D}}$ & $\begin{array}{c}\text { Spot diameter } \\
\text { on detector }\end{array}$ \\
\hline $0.0 \mathrm{~mm}$ & $0.253 \mathrm{~mm}$ \\
$2.0 \mathrm{~mm}$ & $0.253 \mathrm{~mm}$ \\
$5.0 \mathrm{~mm}$ & $0.253 \mathrm{~mm}$ \\
$10.0 \mathrm{~mm}$ & $0.253 \mathrm{~mm}$ \\
\hline
\end{tabular}

As expected, (see Tables $11-5$ and $(1-6)$, the influence of $\Delta \theta_{1}$, and $\Delta Y$ LorB on the lateral shift of the centroid of the focused spol on the detector is quite large. On the other hand, this shift due to $\Delta Y_{f+D}$ is relatively small, as shown in Table 11-7. In Table $11-8$ and $11-9$ the influence of $\Delta Z^{\prime} s$ on the increase of spot diameter show that again, the gystem is highly sensitive to $\Delta Z_{\text {Lor } B}$ and insensitive to $\Delta Z Z_{\ell+D^{\prime}}$

At the second stage of this study, the system used has an $f / f 40$ divergent input beam and the $f / 2$ focusing lens. The primary conceru here was to observe the effects of lateral shift of the sensor package itself on the movement of the 50 percent energy point of the light patch at the beam centering detector. The sensor package was first laterally shifted by $1 \mathrm{~mm}$, and tilted as a whole such that the focus senging detector reads null. As a result, the 50 percent energy point of the light patch at the beam centering detector is also shifted, giving rise to an instrumental error on beam centering. The results are presented in Table $11-10$. The symbols used in this table are exactly those of Figure 11-8. It can be seen that this instrumental error on beam centering is quite large and for this reason, among others, this scheme of bec.- centering was later discarded. Observing the extremely small tilt angles required to correct for the $1 \mathrm{~mm}$ lateral displacement of sensor package, one can deduce that, $2 s$ is in the case of collimated input beam, the sensor package is still insensitive to its own location. 
Table 11-10. Sensitivity of beam centering detectionst: to sensor package positioning; with $\mathrm{f} / 640$ divergent input beam, $/ / 2$ focusing lens and $1 \mathrm{~mm}$ diameter ball

\begin{tabular}{|c|c|c|c|c|}
\hline Cases" & $\begin{array}{l}\mathrm{Y}_{\ell+D} \\
\mathrm{~mm}\end{array}$ & $\begin{array}{c}\Delta \theta_{\ell-D} \text { for } \\
\text { zero reading on } \\
\text { focus sensing detector, } \\
\mu \text { rad }\end{array}$ & $\begin{array}{c}\text { Diameter of } \\
\text { spot on CD, } \\
\text { mm }\end{array}$ & $\begin{array}{l}\text { Lateral shif́t } \\
\text { of } 50 \text { percent } \\
\text { energy point } \\
\text { on CD, } \\
\text { mm }\end{array}$ \\
\hline $\begin{array}{l}d_{2}=3 \mathrm{~cm} \\
F_{2}=100 d_{2}\end{array}$ & 1.0 & 14 & 0.597 & 0.017 \\
\hline $\begin{array}{l}d_{2}=3 \mathrm{~cm} \\
F_{2}=50 d_{2}\end{array}$ & L. 0 & 14 & 0.298 & 0.009 \\
\hline $\begin{array}{l}d_{2}=2 \mathrm{~cm} \\
F_{2}=100 d_{2}\end{array}$ & 1.0 & 17.5 & 0.409 & 0.017 \\
\hline $\begin{array}{l}d_{2}=2 \mathrm{~cm} \\
F_{2}=50 d_{2}\end{array}$ & 1.0 & 17.5 & 0.203 & 0.008 \\
\hline $\begin{array}{l}\mathrm{d}_{2}=1 \mathrm{~cm} \\
\mathrm{~F}_{2}=100 \mathrm{~d}_{2}\end{array}$ & 1.0 & 26.2 & 0.257 & 0.015 \\
\hline $\begin{array}{l}d_{2}=1 \mathrm{~cm} \\
F_{2}=50 d_{2}\end{array}$ & 1.0 & 29.7 & 0.118 & 0.007 \\
\hline
\end{tabular}

"Symbols used here are those of Figure 11-8.

As Lescribed in an appendix in this report, an alternative centering sensor scheme is used in the finalized alignment configuration. 
At the third and final stage of this study, the $f / 2$ focusing lens was replaced by the $f / 1.586$ lens. And attention way given solely to the system sensirivity to target movements. Since the method for initial signal acquisition requires a $7 \mathrm{~mm}$ ball to be placed at the target point, the effects of its $\triangle Y$ and $\Delta Z$ on the motion of the focused spot are also included. The results are given in Table 11-11. 
Table 11-11. Sensitivity of sensor package to movements of the surrogate target: with $f / 640$ divergent input bcam and $f / 1.586$ focusing lens

\begin{tabular}{|c|c|c|c|c|c|c|}
\hline$\underset{\substack{\text { Ball } \\
\text { mm }}}{\underset{\text { Diameter, }}{\text { man }}}$ & $\underset{\mu \mathrm{m}}{\Delta \mathbf{Y}_{\mathrm{B}}}$ & $\underset{\mu \mathrm{m}}{\Delta \mathbf{Z}_{\mathbf{B}}}$ & $\begin{array}{c}\text { Spot Diameter } \\
\text { on Focus } \\
\text { Sensing } \\
\text { Detecior, mm }\end{array}$ & $\begin{array}{c}\text { Lateral Shift of } \\
50 \% \text { Energy } \\
\text { Point on } \\
\text { Focus } \\
\text { Detector } \\
\text { inm }\end{array}$ & $\begin{array}{c}\text { Spot Diameter } \\
\text { on Centering } \\
\text { Detectory, } \\
\text { mm }\end{array}$ & $\begin{array}{c}\text { Lateral Shift of } \\
50^{0 ;} \text { Energy } \\
\text { Point on } \\
\text { Centering } \\
\text { Detector. } \\
\text { mm }\end{array}$ \\
\hline 1.0 & 0 & 0 & o. 085 & 0 & 0.238 & 0 \\
\hline 1.0 & -27.8 & 0 & 0.077 & 1.264 & D. 260 & 0.011 \\
\hline 7.0 & $\mathbf{0}$ & 0 & 0.027 & 0 & 1. 426 & 0 \\
\hline 7.0 & 140 & 0 & 0.130 & -1.417 & 1.507 & -0.259 \\
\hline 7.0 & 0 & 100 & 0.030 & 0 & 1. 432 & 0 \\
\hline 7.0 & 0 & -100 & 0.080 & 0 & 1. 420 & o \\
\hline 7.0 & 140 & 100 & 0.161 & -1.421 & 1.511 & -0.258 \\
\hline
\end{tabular}

As described in an appendix in this report, an alternative centering sessor achome is uscel in the finalized alignment configuration. 


\section{SERVO ANALYSES}

\section{SERVO DESIGN REQUIREMENTS}

The servo design of the certering and pointing mirrors consists basically of selecting a motor and drive that is compatible to the system: requirements. The system requirements that are germane, to the position servo lonps $\equiv$ re listed below.

I. Resolution

The :esolution of the pointing mirror is $0.25 \mu \mathrm{rad}$.

2. Response

The response time of the servo is of secondary importan:e.

3. Maintain position with pcwer off

The position is to be maintained with power off to servo over a low vibration envirunment and subject to heat dissipation from the moto:

The resolution requirements and mairitenance of position with power of $i$ tend to be opposing. The maintenanc ; of position implies some sort of hoiring torque such as friction. The resolution accuracy requires essentially no friction. The synthesis, analysis, and design of the servo is comprised primarily of solving the above somewhat contradictory requirements. The synthes is consists of investigating and illustrating the effects of friction ano compliance. The analysis consists of estimating the stability and the response time of the servo selected and the coupling characteristıcs between the centering and pointing servos. The servo design consists of selecting an electromagnetic sensor and circuits which are compatible to the non-oprical measurement requirements.

\section{SYSTEM SYNTHESIS}

Due to the effects of friction, it is shown in the section that positiuning and holding can only be accomplished with a drive where the friction is isclated at the source of torque. The fricticit error is then reduced by increas ing the drive inertia or enclosing the friction in a magnet:c spring restraint loup. 
This section examines the required friction and compliance requirements of the servo drive. The section is divided into two portions. The first portion deals with servo drives that are fairly rigid such as a low pitch lead screw positioning, a flexure mounted load or a rotating wedge. A lead screw has a minimum of 5 inch-ounce of friction torque at the input. A rotating wedge has a minimum of $\mathbf{5 0}$ inch-ounce of friction torque at the load.

The second portion of the section deals with high motion reduction drives such as a lever drive or a spring drive, both used in conjunction with a ball or lead screw. These drives have a compliance that must be considered.

\section{Rigid Body Synthesis}

The friction is first examined in terms of a very basic conventional rigid body linear direct drive servo. A position loop is shown in Figure 12-1.

The friction acceleration is given by

$$
\text { Friction Acceleration }=\frac{\text { Friction Torque }}{\text { Rigid Body Inertia }}, \mathrm{rad} / \mathrm{sec}^{2}
$$

The relationship between the acceleration constant, bandwidth, and compensation is given by Eq. (12-2) and (12-3).

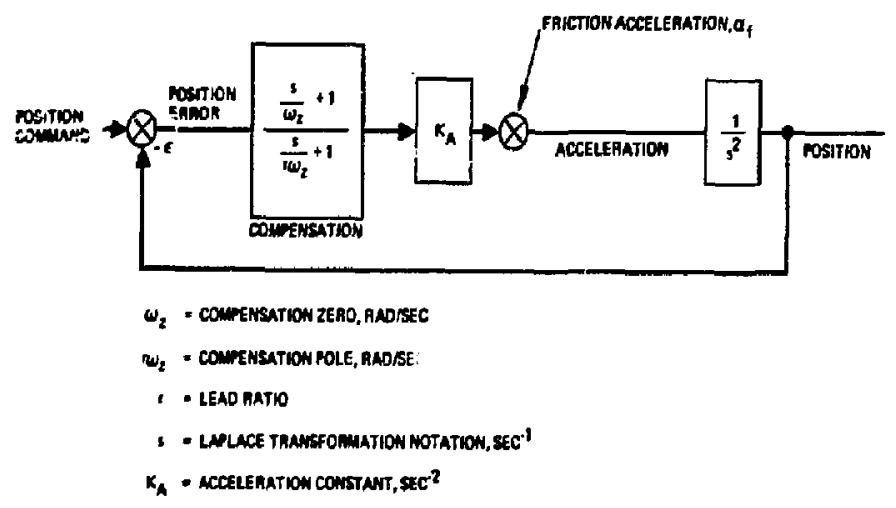

Figure 12-1. Basic position servo loop 


$$
\begin{aligned}
& K_{A}=\omega_{c}^{2} / \sqrt{r} \\
& \omega_{z}=\omega_{c} / \sqrt{r}
\end{aligned}
$$

In both equations, $\omega_{c}$ is the servo bandwidth in radians per second.

The lead ratio of about 6 gives a damping ratio of about 0.7 which gives the best time response for inpui commands. In order to obtain a physical intuition for the friction problem some realistic friction torques, estimated inertias, and required bandwidths are presented below as examples.

The mirror inerita is about 50 in. oz. $\sec ^{2}$. The rigid body inertia when reflected to the load is given by

$$
J=J_{L}+N^{2} J_{r}
$$

where

$$
\begin{aligned}
J_{L} & =\text { Load inertia } \\
N & =\text { Gear ratio } \\
J_{r} & =\text { Motor rotor inertia }
\end{aligned}
$$

The friction torque, $T_{f}$, at the drive that is applifd to the load is given by

$$
T=T_{f} N
$$

For a nominal rigid body gear ratio, the total load inertia may be doubled such that $\mathrm{J}_{\mathrm{L}} \approx 100$ in. $0 \mathrm{z}, \mathrm{sec}^{2}$. For a drive that has bearings or a lead screw, the friction at the load may be a minimum of 20 , $\mathrm{n}, \mathrm{oz}$.

The transier function for friction acceleration input to position error out is given by 


$$
\begin{aligned}
\frac{\epsilon}{\alpha_{f}} & =\frac{\frac{1}{s^{2}}}{1+\frac{\left(\frac{s}{\omega_{z}}+1\right) K_{A}}{s^{2}\left(\frac{s}{r \omega_{z}}+1\right)}} \\
& =\frac{K_{A}-1\left(\frac{s}{r \omega_{z}}+1\right)}{\left(\frac{s^{2}}{\omega_{c}^{2}}+2 S \frac{s}{\omega_{c}}+1\right)\left(\frac{s}{\omega}+1\right)}
\end{aligned}
$$

A frequency response of the amplitude of Eq. (12-7) is shown in Figure 12-2. The amplitude of the position error over the bandwidth of the servo is

$$
\epsilon=\frac{\alpha_{f}}{\bar{k}_{A}}, \quad|s|<\omega_{c}
$$

Therefore for an inertial load of 100 in. $02 . \sec ^{2}$ and a friction torque of $20 \mathrm{in}, o z$, the required acceleration for a resolution of $0.25 \mu \mathrm{rad}$ is $2 \times 10^{6} / \mathrm{sec}^{2}$, or from $E q .(12-3)$ the equivalent bandwidih is given by

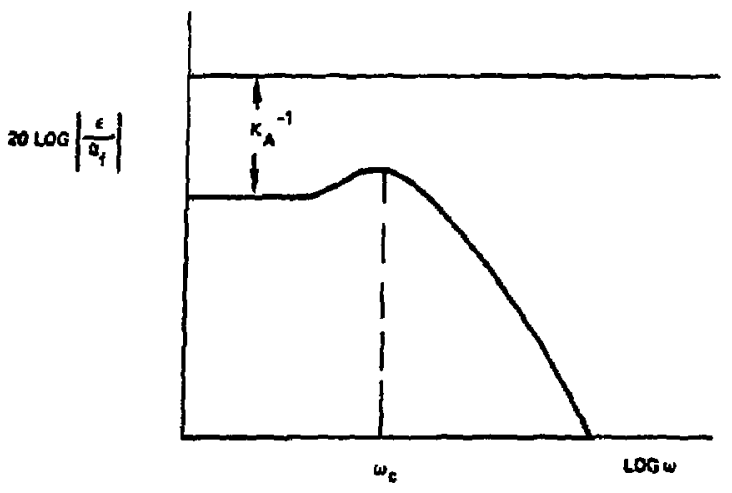

Figure 12-2, Frequency sesponse for equation (12-7) 


$$
\begin{aligned}
\omega_{c} & =\left(2 \times 10^{6}\right)^{1 / 2}(6)^{-1 / 4} \\
& =2200 \mathrm{rad} / \mathrm{sec} \\
& =350 \mathrm{~Hz}
\end{aligned}
$$

A bandwidth of $350 \mathrm{~Hz}$ is impossible to achieve for this particular strucsure. A bandwidth of $20 \mathrm{~Hz}$ would be difficull to achieve with any structure that has some sort of amplitude reduction between the drive and the load. $A$ plot of bandwidth and tolerable friction torque at the load is shown in Figure 12-3. It may be noted that realistic friction torque and bandwidth of a

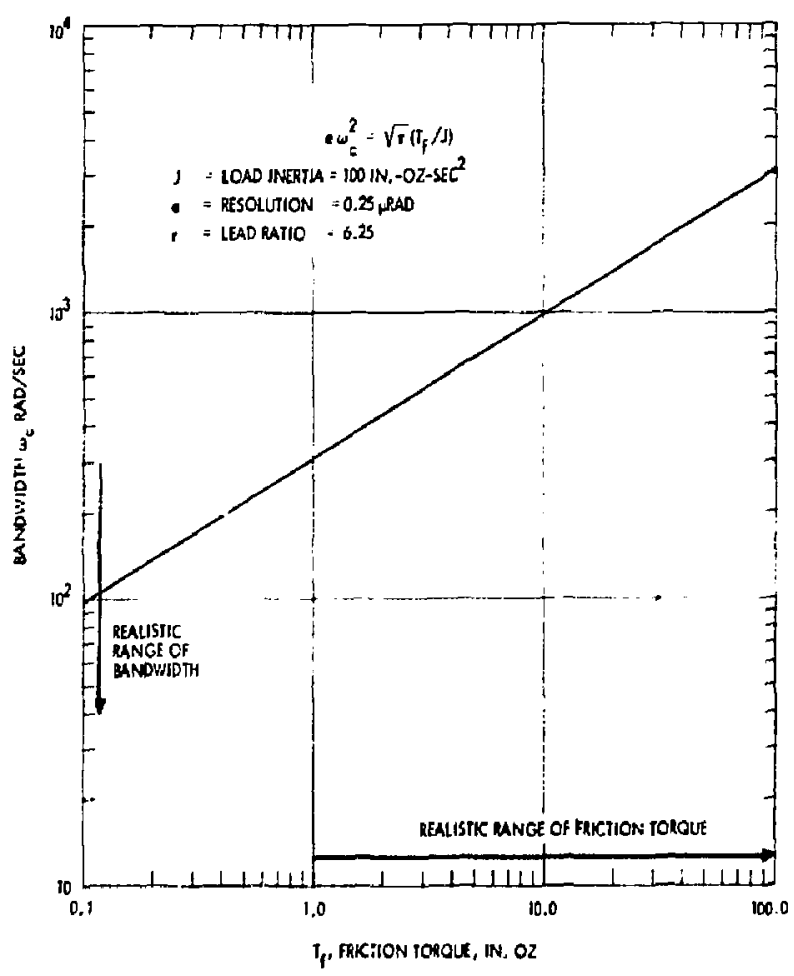

Figure 12-3. Bandwidth as a function of friction torque at load for linear servo with rigid drive 
linear conventional servo are, for this positioning accuracy, incongruous. From the above development two points are made clear:

1. High loop gain must be achieved to reduce friction disturbance.

2. The drive must be selected that results in a friction torque at the load that produces a position error within the resolution.

The fact that the above requirements may not be met does not preclude meeting the resolution requirements. If the energy that the inertia has when entering the friction dead zone is equal to the (friction torque) times the (friction error-resolution), then the load will come to rest within reso.ution. This type of control relies on random phenomera and is not considered good servo design. Also applying a dither torque by either the operatur or fixed superimposed signal results in reduction of effective friction torque. The superimposed dither signal results in increased power dissipation and wear.

High loop gain without excessive bandwidth implies nonlinear bang-bang control. A bang-bang controller with a rigid loac is shown in Figure 12-4. The principle of bang-bang control is explained in any optimal control or nonlinear control text ${ }^{(\text {Ref. } 1,2)}$. Constant torque is applied and reversed when

$$
\frac{\text { (2)(torque) }}{\text { (Inertia) }}=-|\dot{\dot{e r r}}| \quad(\text { érr }
$$

such that the error is reduced to zero in a minimum time.

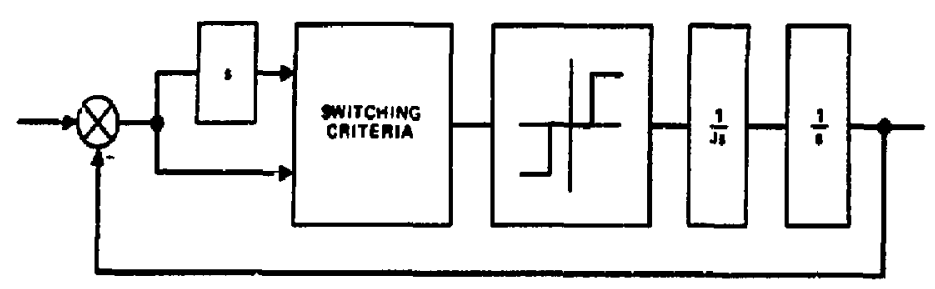

Figure 12-4. Bang-bang controller with rigid load 
The dead space is equal to the resolution of the servo. When the load tends to be nonrigid, there is cumpliance between the torque source and the load such that the commanded acceieration at the motor is not identical to that of the load anci a limit cycle will exist. Also in a flexure-supported load, the flexure torque biases the load acceleration.

The minimum structural compliance is now determined to see if "bangbang" control may possibly be used. A simple general mechanical diagram is shown in Figure 12-5. This figure describes a rotating wedge drive when $k_{f}=0$, and a flexure-mounted load when $T_{f \ell}=0$. Damping is not included but is presumed later.

\section{Flexure with Ball Screw}

From Figure 12-5, it may be seen intuitively that these are two sources of instability. The applied to rque may compress the drive spring and excite the spring mass system at its resonant frequency with a resultant amplitude that is greater than the resulution. The load friction may also excite the system. These sources of instability a re investigated by developing the equations of motion.

\section{$\Sigma$ Torques on Drive Inertia}

$$
T=J_{s} s^{2} \theta_{r}+I_{f r}+k_{s} \frac{\left(\theta_{n}-\theta_{l}\right)}{N}+\frac{T_{f d}}{N}
$$

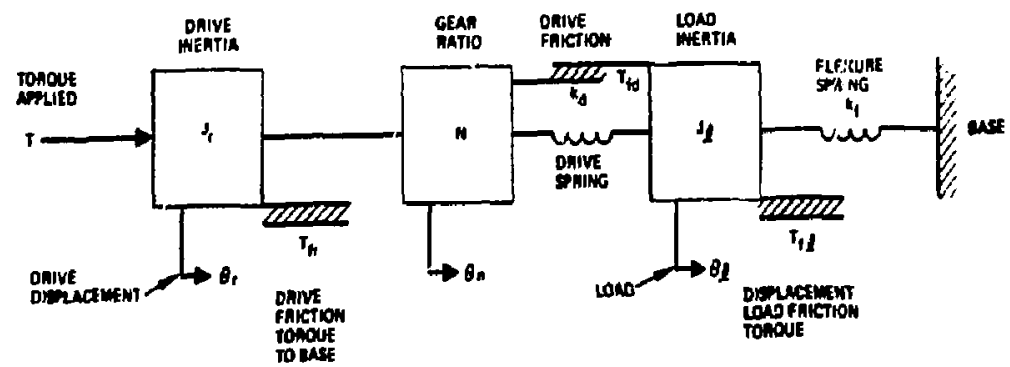

Figure 12-5. Mechanical diagram of flexible drive with friction 


\section{$\Sigma$ Torques on Load Inertia}

$$
\mathrm{T}_{\mathrm{fd}}+\mathrm{k}_{\mathrm{d}}\left(\theta_{\mathrm{n}}-\theta_{l}\right) \cdot \mathrm{J}_{l} \mathrm{~s}^{2} \theta_{l} \cdot \mathrm{T}_{\mathrm{fl}}+\mathrm{k}_{\mathrm{i}} \theta_{l}
$$

Motion Reduction

$$
\theta_{\mathbf{r}} \quad \because \theta_{\mathrm{n}}
$$

Eq. (12-11) through $(12-13)$ are further described with the aid of the block diagram of Figure 12-6.

The matrix of Eq. $(12-11)$ through $(12-13)$ is given below in Eq. $(12-14)$.

$$
\left[\begin{array}{ccc}
J_{r}^{2} & k_{d} N & -k_{d} N \\
-1 & N & 0 \\
0 & -k_{d} & J_{l} s^{2} \cdot k_{f}+k_{d}
\end{array}\right]\left[\begin{array}{l}
\theta_{r} \\
\theta_{n} \\
\theta_{l}
\end{array}\right]:\left[\begin{array}{c}
T-T_{f r}-T_{f d} X \\
0 \\
T_{f d}-T_{i \ell}
\end{array}\right]
$$

The characteristic equation of Matrix Eq. $(12-14)$ is given below in Eq. $(12-15)$ in the transfer function from torque applied to the drive inertia to load position.

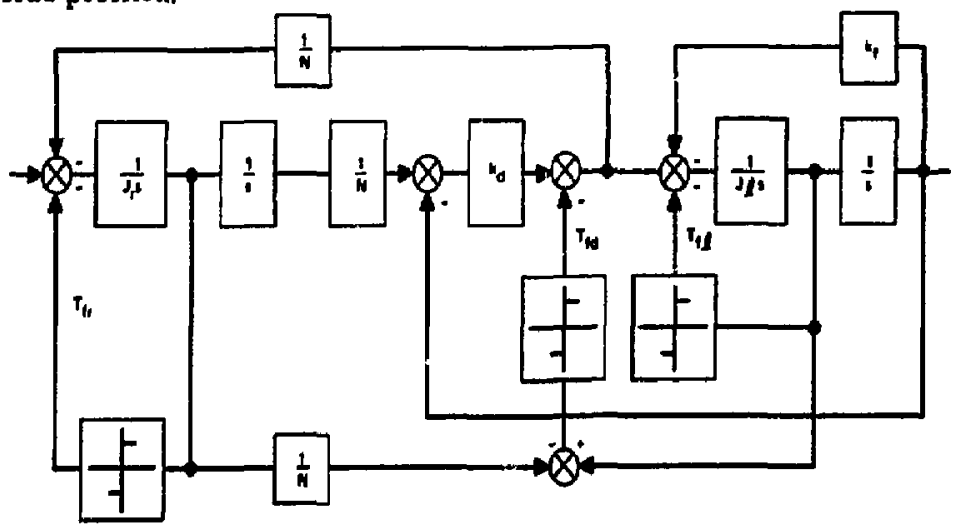

Figure 12-6. Block diagram of spring mass model of Figure 12-5, 


$$
\frac{\theta_{l}}{T-T_{f r}-T_{f d} / N}=\frac{N / k_{t}}{\frac{J_{l} N^{2} J_{r}}{k_{f} k_{d}} s^{4}+\left[N^{2} T_{r} \frac{\left(k_{f}+k_{d}\right)}{k_{f} k_{d}}+\frac{J}{k_{f}}\right] s^{2}+1}(12-15)
$$

If drive spring is very much greater than flexure spring

$$
\frac{L}{T-T_{i f}-T_{f d^{\prime}} N}=\frac{N / k_{f}}{\left(\frac{J_{l}}{k_{f}} s^{2}+1\right)\left(\frac{N^{2} g_{r}}{k_{d}} s^{2}+l\right)}
$$

The friction torque at the mutsr must be greater than the required flexure to rque at maximum load deflection.

$$
I_{\text {applied at motor }} \geq 2\left(\frac{\theta_{l \max } k_{f}}{N}\right)
$$

The dynamic deflection of the drive spring at the load resonance is a function of mechanical damping, $\delta$, and applied torque on the spring.

$$
\frac{\theta_{\ell} \max ^{k}\{}{2 \delta} \leq k_{d} \text { (resolution) }
$$

From inequality Eq. $(12-18)$ it looks as if it may be possible to orercome the friction problem theoretically by mears of bang-bang control. Inequality Eq. $(12-18)$ is now evaluated for some realistic parameters.

The minimum flexure spring rate is determined by the support requirements. For the support of interest, the flexure frequency is about $4 \mathrm{~Hz}$ such that for $J_{l}=50$ in-02-sec ${ }^{2}, k_{i}$ is about 30,000 in-02/rad from Eq. (12-17). The maximum load deflection is $10 \mathrm{mrad}$ and a worst case damping, $\delta$, of 0.05 is assumed. $k_{d}$ is thereiore given by Eq. $(12-19)$. 


$$
\begin{aligned}
k_{d} & =\frac{(0.01 \mathrm{rad})\left(3 \times 10^{4} \mathrm{in-0z} / \mathrm{zad}\right)}{(2)(0.05)\left(0.25 \times 10^{-6}\right)} \\
& =1.2 \times 10^{10} \mathrm{in-0z} / \mathrm{rad}
\end{aligned}
$$

This value of $k_{d}$ corresponds to a mechanical bandwidth of

$$
\begin{aligned}
& \omega_{n}=\sqrt{\frac{1.2 \times 10^{10} \mathrm{in-oz} / \mathrm{rad}}{50 \mathrm{in}-02-\mathrm{sec}^{2}}} \\
& =15,500 \mathrm{rad} / \mathrm{sec}
\end{aligned}
$$

The above bandwidth is physically uncealizable, but it is still interesting to note that if the required flexure spring rate were small enough, ideal bang-bang control could solve the friction problem.

\section{Wedge Servo}

The wedge servo is capable of being fairly rigid. However, the friction torque at the load tends to be high. The minimum friction torque expected is about 50 in-oz at the load. This friction torque applied to the drive spring must produce less displacement than the resolution at the load natural frequency

$$
\frac{T}{k_{d}^{2 \delta}} \leq \text { Resolution }
$$

If a worst case damping of 0.05 is assumed,

$$
k_{d}=\frac{50 \mathrm{in}-0 \mathrm{z}}{(2)(0.05)\left(0.25 \times 10^{-6} \mathrm{rad}\right)}=2 \times 10^{9} \mathrm{in-0z} / \mathrm{rad}
$$


The above drive spring rate is equivalent to a bandwidth of

$$
\omega_{n}=\sqrt{\frac{2 \times 10^{9}}{50}}=6200 \mathrm{rad} / \mathrm{sec}
$$

The above mechanical resonance is also nut physically realizable.

\section{Conciusion}

It has been shown that by trying to maintain the structural rigldity within a physically realizable realm dues not solve the friction problem.

\section{High Motion Reduction Servo}

A means of reducing the friction reflected to the load is to hare the friction isolateri near the torque surce and then have a large notion reduction by means of flexures or springs. These drives tend to be quitf compliant such that the stability must be carefully exaluated.

The friction acceleration at the load is given by

$$
\alpha_{i}=\frac{T_{f} N_{r}}{x_{r}^{2} J_{r}+J+\sum_{i} N_{i}^{2} J_{i}}
$$

where

$$
\begin{aligned}
& T_{f}=\text { Friction torque at motor } \\
& N_{r}=\text { Motion reduction ratio from motor } \\
& N_{i}=\text { Motion reduction ratio Irom } i^{\text {th }} \text { lever } \\
& J_{r}=\text { Motor inertia } \\
& J=\text { load inertia } \\
& J_{i}=\text { Inertia of } i^{\text {th }} \text { lever }
\end{aligned}
$$


When $x \rightarrow \infty$

$$
a_{i}=\frac{T_{f} J^{I}}{T^{2}}
$$

Figure 12.7 is a plut of Eq. 112-24, with the assumption that the drive : rigid.

$$
a_{1}-\omega_{c}^{2} i \sqrt{x}
$$

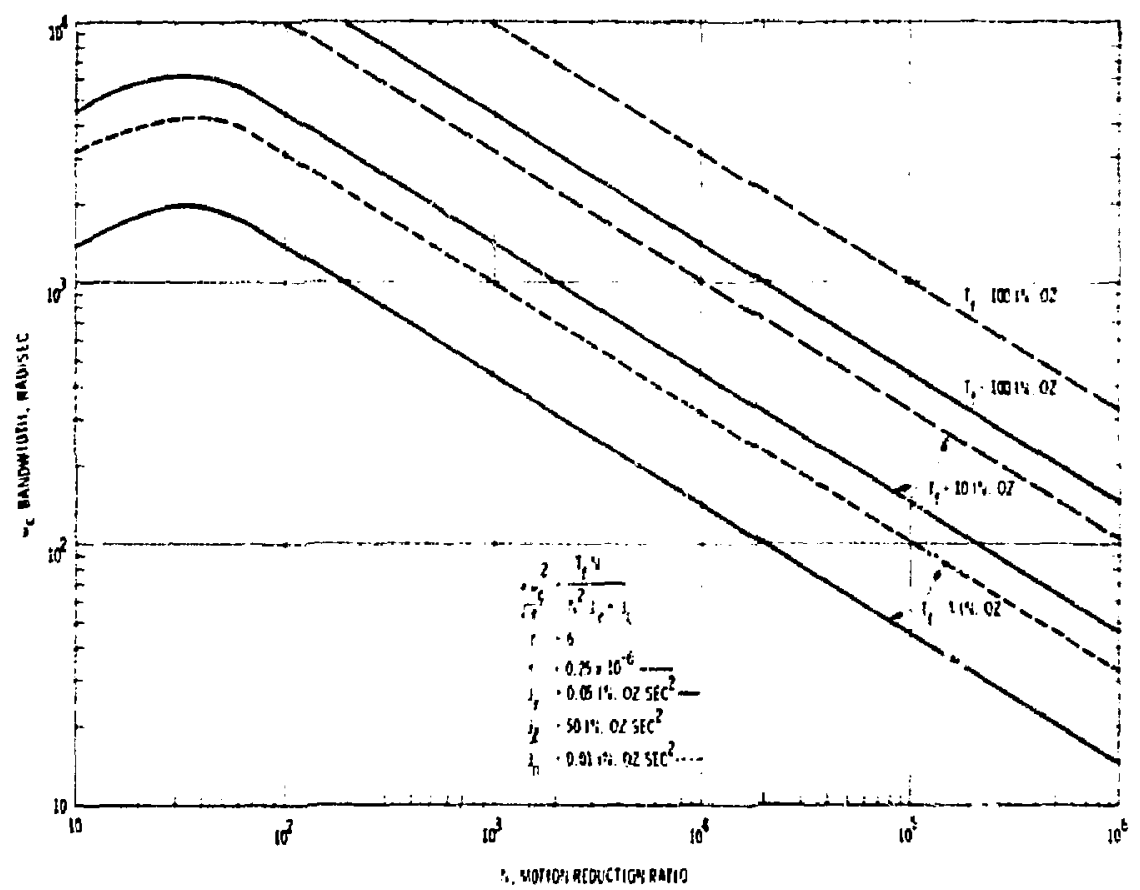

Figure 12-7. Plat of bandwidth versus motion exduction ratio of liteve rigid body servo with all friction located at drive 
where

$$
\begin{aligned}
& r=\text { resolution } \\
& \tilde{c}_{c}=\text { bandwidth } \\
& r=\text { lead ratio }
\end{aligned}
$$

The curves of Figure 12- 7 are useful in determining the meshanical resinance of the motion reduction ratio mechanism. A ruie of thumb is that the lowest mechanical resunance is six tines the servo bandwiath. For example. if

$$
\begin{aligned}
& J_{r}=0.05 \mathrm{in}-a z-\sec ^{2} \\
& \therefore=10^{6} \\
& J_{l}=50 \mathrm{in}-0 z-5 \sec ^{2} \\
& I_{f}=1.0 \mathrm{in}-(1)<
\end{aligned}
$$

From Figure 12-i the minimun esunance of the drive and the load is arat/sec.

The mechanical compliance problem is illustrated by analyzing the spring mass system shown in Figure 12-B. A rigid base is assur.ed in the Gigure, and damping is considered later The example shosen is that of a

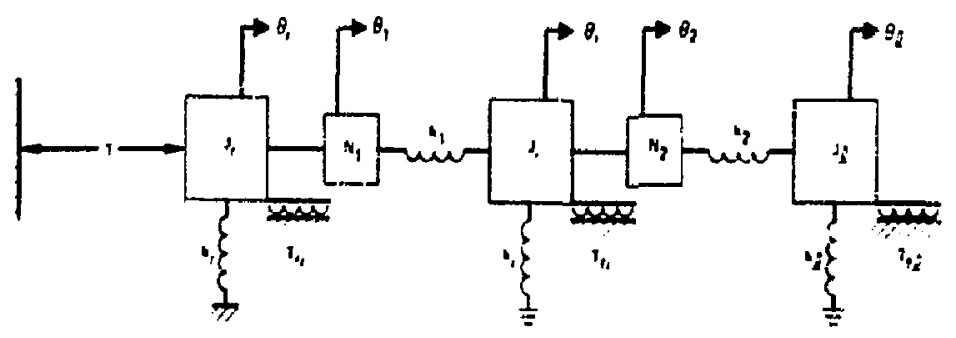

Figure 12-8. Mechanicaj luniped spring mass diacram 
systerc comprised of two motion reductions, three inertias, and wo springs or flexures.

The mcchanical parameters are defined bel.w.

$J_{r}=M_{2}$ itr rotor inertia plus any inertia rigidly attached to rotor

$T_{i r}=$ Friction torque between motor rotor and base

$k_{r}$ - Spring rate between rotor inertia and base, torque/displacement (stepper motor only)

$\sigma_{r}=$ Rotory displacementi of motor rotor

$\lambda_{1}=$ First motion reduction ratio

$h_{1}=$ Spring rate between rotor and first gear ratio

$0_{1}=$ Rotary displacement al input to first motion reduction

$s_{j}=$ Intermediate ineria

$f_{i}:$ Rotary displacement of intermediate inertia

$k_{i}=$ Spring rate between internediate tnertia and base

$T_{21}=$ Friction torque between intermeriate inertia and base

$k_{2}=$ Spring rate betwern intermediate inertia and second motion reduction, forque/displacement

$0_{2}$ = Rulary displacemert at inpat 10 sesond motion reduction

$X_{1}=$ Second motion reduction ratio

$J_{k}=$ Load inertia

$k_{p}=$ Spring rate between luad and base 
$T_{i l}=$ Friction torque between load and base

$\theta_{1}=$ Rotary displacement at load

The equations of motion of the various inertias and displacernent relationships a re given below in Laplace transform notation.

Rotor Inertia

$$
\left.T-10_{r}-\theta_{1}\right) k_{1}=J \mathbf{r}_{\mathbf{r}} \mathbf{s}^{2} \div k_{r} \theta_{r} \div \Gamma_{f}
$$

Firsl Motion Reduction

$$
0_{1}=N_{1} c_{i}
$$

Intermediate Inertia

$$
10_{i}-\theta_{1} \mid k_{1} N_{1}=J_{i} \theta_{i} s^{2}+k_{i} \theta_{i}+\left(\theta_{i}-\theta_{2}\right) i k_{2}+T_{f i}
$$

Second Motion Reduction

$$
\theta_{2}=s_{2} \theta_{l}
$$

\section{Load lneatia}

$$
\left(0_{1}-0_{2}\right) k_{2} N_{2}=J_{l} 0_{l} s^{2}+k_{l} \theta_{l}+T_{i l}
$$

A matrix of the abore equations is given in Eq. (12-32). A blucle diagram of the trive without friction sources is shown in Figure 12-9. 


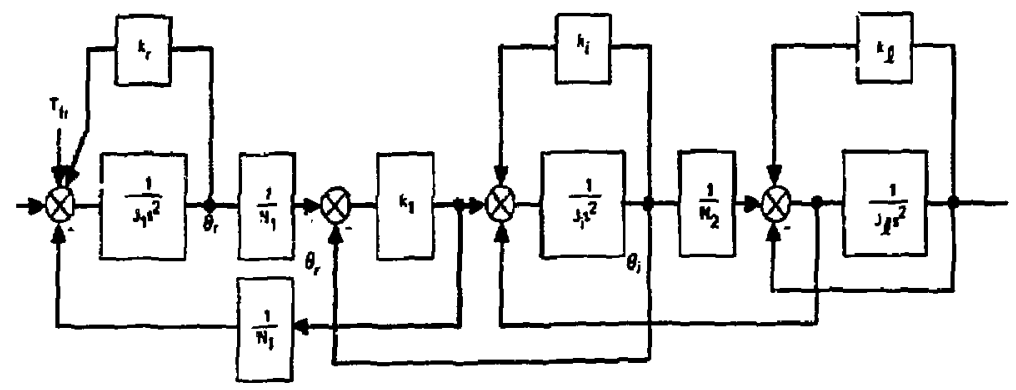

Figure 12-9. Block diagram of Figure 12-8

$\left[\begin{array}{ccc}J_{r} s^{2}+k_{r}+\frac{k_{1}}{N_{1}^{2}} & \frac{k_{1}}{N_{1}} & 0 \\ -\frac{k_{1}}{N_{1}} & J_{1} s^{2}+k_{1}+k_{i}+\frac{1}{N_{2}^{2}} & -\frac{k_{2}}{N_{2}} \\ 0 & -\frac{k_{2}}{N_{2}}\end{array}\right]\left[\begin{array}{l}\sigma_{i} \\ \theta_{i} \\ J_{s}^{2}+k+k_{2}\end{array}\right]=\left[\begin{array}{l}-T_{f i} \\ \theta \\ -I_{f}\end{array}\right]$

$(12-32)$

The transfer function for torque in to load position out of Matrix

Eq. $(12-32)$ is 


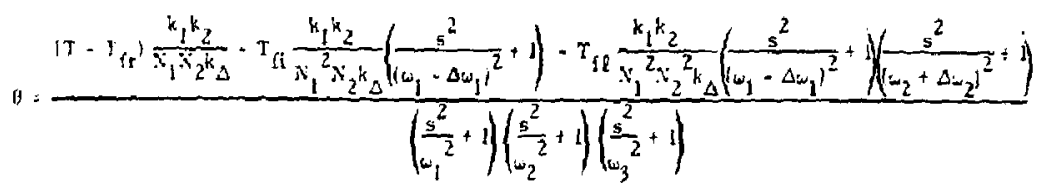

where

$$
\begin{aligned}
k \Delta= & k_{r} k_{1} k_{l}+k_{r} k_{i} k_{l}+\frac{k_{r} k_{2} k_{\ell}}{N_{2}^{2}}+\frac{k_{1} k_{i} k_{l}}{N_{1}^{2}}+\frac{k_{1} k_{2} k_{l}}{N_{1}^{2} N_{2}^{2}}+k_{2} k_{r} k_{1} \\
& +k_{r} k_{i} k_{2}+\frac{k_{l} k_{i} k_{2}}{N_{1}^{2}}
\end{aligned}
$$

and where

$$
\dot{i n}_{1}^{2}=\frac{k_{11}}{J_{11}} .
$$

primarily due tu resonance about motor. $\approx 0$ for conventional servo mutor

$$
\begin{aligned}
& k_{11}=k_{r}+\frac{1}{N_{1}^{2}}\left[\left(\frac{1}{k_{1}}+\frac{1}{k_{i}}\right)+\left(\frac{1 / N_{2}^{2}}{\left.\frac{1}{k_{2}}+\frac{1}{k_{2}}\right)}\right]\right. \\
& J_{11}=\left[\frac{1}{J_{r} N_{1}^{2}}+\left(\frac{1}{N_{2}^{2} J_{i}}+\frac{1}{7}\right)^{-1}\right]^{-1}
\end{aligned}
$$




$$
\omega_{2}^{2}=\frac{k_{22}}{J_{22}}
$$

Primarily due to intermediate inertia and connecting springs

$$
\begin{gathered}
\mathrm{k}_{22} \approx \mathrm{k}_{\mathrm{i}}+\frac{1}{\frac{1}{\mathrm{k}_{\mathrm{r}} \mathrm{N}_{1}^{2}+\frac{1}{\mathrm{k}_{1}}}+\frac{1}{\mathrm{~N}_{2}^{2}\left(\frac{1}{\mathrm{k}_{2}}+\frac{1}{\mathrm{k}_{l}}\right)}} \\
\mathrm{J}_{22} \approx \frac{1}{1 / \mathrm{N}_{1}{ }^{2} \mathrm{~J}_{\mathrm{r}}+1 / \mathrm{J}_{\mathrm{i}}+\mathrm{N}_{2}{ }^{2} / \mathrm{J}_{l}}
\end{gathered}
$$

and where

$$
\omega_{3}=\frac{k_{33}}{J_{33}}
$$

prima rily due to load isertia and flexure

$$
\begin{aligned}
& k_{33}=k_{\ell}+\frac{1}{\left[\left(\frac{1}{k_{2}}+\frac{1}{N_{2}^{2} k_{i}}\right)+\left(\frac{N_{2}^{2}}{\left(\frac{1}{k_{i}}+\frac{1}{N_{1}^{2} k_{r}}\right)}\right)^{-1}\right.} \\
& J_{33}=\left[\frac{1}{J_{l}}+N_{2}^{2}\left[\frac{1}{\left(1 / J_{i}\right)+\left(1 / N_{l}^{2} J_{r}\right)}\right]^{-1}\right]^{-1}
\end{aligned}
$$

The compliance problem has now been demonstrated in general. Now let us examine the use of a conventional servo motor $\left(k_{\mathbf{r}}=0\right)$ and a stepper motor $\left(k_{r} \neq 0\right)$ whose step is within the resolution necessary. When a 
conventional servo motor is used, $w_{1}$ is essentially zero. When a stepper motor is used, $\omega_{1} \gg \omega_{2}, \omega_{3} . \omega_{3}$, which is primarily the load ilexure resonance, is optimally designed for load support and flexure is about $4 \mathrm{~Hz}$. Now a conventional servo loop may be closed at about $10 \mathrm{~Hz}$ if the intermediate structure has resonances (i. e., $w_{2}$ ' greater than ó 0 fiz or may be closed at less than $2 / 3 \mathrm{~Hz}$ (from Figure 12-7) if load damping is about 0.5

$$
\text { Now, for } \begin{aligned}
& k_{r}=0 \\
& w_{2} \approx\left[\frac{N_{1}^{2} J_{r} J_{i} \cdot N_{1} J_{r}+J_{i}}{\left(k_{1}+k_{i}\right)}\right]^{-1} \\
& J_{5}=0.01 \text { in-oz-sec } \\
& k_{1} \approx 1.5 \times 10^{6} \text { in-oz, rad for a ball screw, } \gg k_{i} \\
& N_{1} \approx 500 \text { for a ball screw }
\end{aligned}
$$

therefore

$$
\omega_{2}=\sqrt{\frac{1.5 \times 10^{6}}{J_{i \max }}}=(60112 \pi) \mathrm{rad} \sec
$$

$J_{i \max }=10$ in-oz-sec ${ }^{2}$

An intermediate inertia, $I_{i \max }$ of $10 \mathrm{in-oz- \operatorname {sec } ^ { 2 }}$ is insufficient for structural rigidity in a series of levers. If a stepper motor were used, the spring rate of the intermediate structure would be increased and stru.tural rigidity could possibly be mainta ined. The dynamics of the stepper would have to 'ie matched with the loars.

It has now been shown that by means of levers and the use of a conventional servo motor, a low bandwidth servo loop will meet system requirements. The bandwidth should be less than the lowest resonant frequency of the drive by about a factor of $b$. This type ui servo has been develuped and desc ribed in Reference 3. 
In the above development, if the intermediate inertia could have been made small - such as in a spring ratio system - a higher bandwidth system may have been closed. The spring ratio position is now investigated.

\section{Spring Ratio Servo}

A motion reduction by means of balancing a light spring against a heavy spring is appealing from a packaging standpoint. The spring servo is comprised of the load mounted on a flexure spring, a motor, a light spring, a ball screw or a lead screw. A mechanical spring inertia diagram is presented in Figure 12-10.

The mechanical parameters are

$J_{r}=$ Motor shaft inertia

$J_{b}=$ Inertia at input to ball or leal

$\mathrm{N}=$ Equivalent gear ratio of ball or lead screw

$k_{f}=$ Fine spring rate

$J_{\ell}=$ Load inertia

$k_{c}=$ Flaxure spring

$T_{f}=$ Friction torque at ball or lead screw

$k_{r}=$ Motor rotor spring rate (stepper motor only)

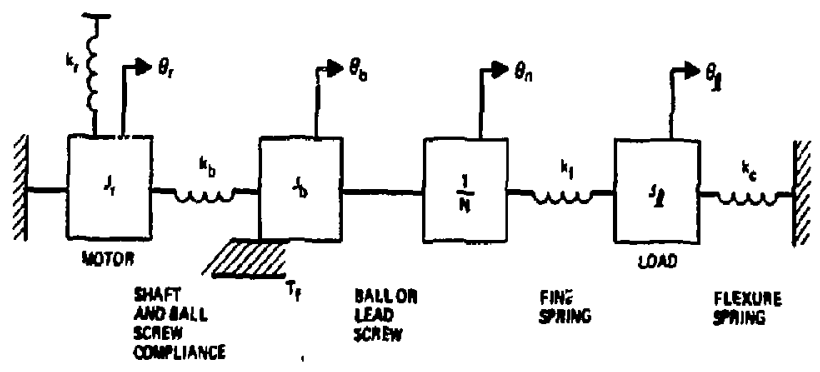

Figure 12-10. Mechanical diagram of spring ratio system 
The equations of motion of Figure 12-10 a re given below.

$\sum$ Torques on Motor Shaft

$$
T_{1}=J_{r} s^{2} \theta_{r}+k_{b}\left(\theta_{r}-\theta_{b}\right)+k_{r} \theta_{r}
$$

$\Sigma$ Torques on Ball Screw Inertia

$$
k_{b}\left(\theta_{r}-\theta_{b}\right)=J_{b} s^{2} e_{b}+k_{f}\left(\theta_{n}-\theta_{l}\right) \frac{1}{N^{2}}+T_{f}
$$

$\underline{\Sigma \text { Torques on Load }}$

$$
\left(\theta_{r}-\theta_{l}\right) k_{i}=J_{l} s^{2} \theta_{l}+k
$$

The matrix of Eq. (12-46) through $(12-48)$ is given by Eq. $(12-49)$.

$$
\left[\begin{array}{ccc}
J_{r} s^{2}+k_{b}+k_{r} & -k_{b} & 0 \\
-k_{b} & J_{b} s^{2}+k_{b}+\frac{k_{f}}{N^{2}} & -k_{f} / N \\
0 & -k_{f} / N & J s^{2}+k_{c}+k_{f}
\end{array}\right]\left[\begin{array}{l}
\theta_{r} \\
\theta_{b}
\end{array}\right]=\left[\begin{array}{l}
T \\
T_{f} \\
0
\end{array}\right] .
$$

$(12-49)$

A block diagram of Eq. $(12-46)$ through (12-48; is shown in Eigure 12-11. 


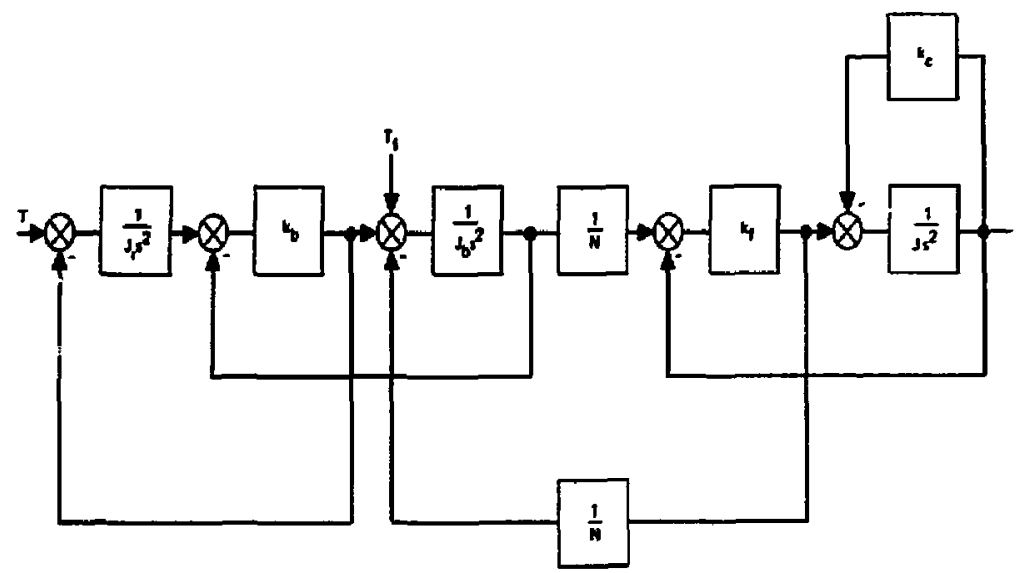

Figure 12-1i. Spring servo load block diagram

Expansion of the matrix of Eq. (12-49) gives the characteristic equation of the system.

$$
\begin{aligned}
\Delta_{r}= & J_{r} J_{b} J_{l} s^{6}+\left[\left(k_{r}+k_{b}\right) J_{b} J_{l}+\left(k_{b}+\frac{k_{f}}{N^{2}}\right)\left(J_{l} J_{r}\right)+\left(k_{c}+k_{f}\right)\left(J_{b} J_{r}\right)\right] s^{4} \\
& +\left[\left(k_{r}+k_{b}\right)\left(k_{c}+k_{f}\right) J_{b}+\left(k_{b}+k_{r}\right) \frac{k_{f}}{N^{2}} J_{l}+\left(k_{b} k_{c}+\frac{k_{f}}{N_{b}^{2} k_{c}}+k_{f} k_{b}\right) J r s^{2}\right. \\
& +\left(k_{r} k_{b} k_{c}+k_{r} \frac{k_{f}}{N^{2}} k_{c}+k_{b} \frac{k_{f}}{N^{2}} k_{c}+k_{f} k_{b} k_{r}\right)
\end{aligned}
$$

When a stepper motor is not used

$$
\begin{aligned}
\Delta_{r=0}= & J_{r} J_{b} J_{B}^{6}+\left[k_{b} J_{b} J_{l}+\left(k_{b}+\frac{k_{f}}{N^{2}}\right) J_{r} J_{l}+\left(k_{c}+k_{f}\right) J_{b} J_{r}\right] s^{4} \\
& +\left[k_{b}\left(k_{c}+k_{f}\right) J_{b}+k_{b} \frac{k_{f}}{N^{2}} J_{l}+\left(k_{b} k_{c}+\frac{k_{f}}{N^{2}} k_{c}+k_{f} k_{b}\right) J_{r}\right] s^{2}+k_{b} \frac{k_{f}}{N^{2}} k_{c}
\end{aligned}
$$


From Matrix Eq. (12-49), the transfer function of torque in to load position out is given by

$$
\theta_{\ell}=\frac{T\left(k_{b} k_{f} / N\right)-T_{f}\left(k_{f} / N\right)\left(J_{r} s^{2}+k_{r}+k_{b}\right)}{\Delta_{r}}
$$

Now certain simplifying assumptions are made

$$
\begin{aligned}
& J \gg J_{b} \approx J_{r} \\
& k_{b} \gg \frac{k_{f}}{N^{2}} \\
& k_{c} \gg k_{f} \\
& k_{b} \gg k_{r}
\end{aligned}
$$

Eqs. (12-50) and (12-51) are now rewritten after making use of the inequalities Eqs. (12-53) through (12-56)

$$
\begin{aligned}
\Delta_{r}= & J_{r} J_{b} J_{l} s^{6}+\left[k_{b}\left(J_{l} J_{b}+J_{\ell} J_{r}\right)+k_{c} J_{b} J_{r}\right]^{4} \\
& +k_{c}\left(k_{r}+k_{b}\right) J_{b}+\left(k_{b}+k_{r}\right) J+k_{b} k_{c} J_{r} s^{2}+k_{r} k_{b} k_{c} \\
\Delta_{r=0}= & J_{r} J_{b} J_{l} s^{6}+\left[k_{b}\left(J_{l} J_{b}+J_{l} J_{r}\right)+k_{c} J_{b} J_{r}\right] s^{4} \\
& +k_{b} k_{c}\left(J_{b}+J_{r}\right) s^{2}+k_{b} \frac{k_{f}}{N^{2}} k_{c}
\end{aligned}
$$


The above equations are used in determining the resonant frequencies of the drive and the inherent friction torque disturbance attenuation of the stepper motor. The static friction torque at the load for a stepper motor, obtained from Eqs. (12-52) and (12-57) is

$$
\epsilon_{s=0}=T_{f} \frac{k_{f}}{k_{c} k_{r} N} \text { (for stepper motor) }
$$

The static friction error at load of the conventional servo motor is obtained from Eqs. (12-52) and (12-58) and is simply that which was stated previously.

$$
\xi_{s=0}=T_{f} \frac{N}{k_{c}} \text { (ior conventional motor) }
$$

Eqs. (12-59) and (12-60) are now evaluated for some realistic parameters.

A ball screw has a friction torque of about 1 in-oz at its input and an equivalent $\mathrm{N}$ of 500 . The coarse spring may have a spring rate of about $2 \times 10^{5} \mathrm{in-02} / \mathrm{rad}, \mathrm{k}_{r}=6000 \mathrm{in-02} / \mathrm{rad}$ for a stepper motor. The fine-tocoarse spring rate ratio is about 1000 . The respective errors are then given as

$$
\begin{aligned}
& c_{\mathrm{s}=0}=0.66 \times 10^{-9} \mathrm{rad} \text { for a stepper motor } \\
& c_{\mathrm{s}=0}=0.25 \times 10^{-2} \mathrm{rad} \text { for a conventional motor }
\end{aligned}
$$

The static error is reduced by the servo loop gain. To achieve a resolution of $0.25 \mu \mathrm{rad}$ requires a gain of $10^{4}$ or an equivalent bandwidth of about $20 \mathrm{~Hz}$. The spring rate of a ball servo is about $2 \times 10^{6} \mathrm{in-oz} / \mathrm{rad}$ at the output such that it may be shown from Eq. (12-58) that one of the dominant resonances occurring between the drive and load is

$$
\sqrt{\frac{k_{b}}{I}}=200 \mathrm{rad} / \mathrm{sec}
$$


The above resonance precludes a $20 \mathrm{~Hz}$ servo loop.

The feasibility of using a stepper motor with a spring ratio drive has now been demonstrated. The dynamics of the stepper motor and load must now be investigated.

\section{Srepper Motor Load Dynamics}

The stepper motor as a servo element is described in Appendix $L$. It is basically an incremental positioning device which positions its rotor bidirectionally at a fixed repetition rate. Intuitively, the pulse rate command to the motor cannot be greater than the time it requires to make one step. This time is determined by the basic kinematic equation below

$$
\theta=\frac{1}{2} \alpha t^{2}
$$

where

$$
\begin{aligned}
& \theta=\text { angular displacement } \\
& t=\text { time } \\
& \alpha=\text { acceleration }
\end{aligned}
$$

If a time optimal response is assumed, such that peak acceleration is commanded over the first half of the step and peak de-acceleration over the second half of the pulse, the time to complete one step is given by

$$
T_{0}=2 \sqrt{\frac{\theta_{s}}{\sigma}}
$$

The acceleration for a rigid load is given by

$$
a=\frac{\text { Peak Torque }}{\left(\text { Load Inertia) } / \mathrm{N}^{2}+\right.\text { Rotor Inertia }}
$$


Eqquations $(12-64)$ and $(12-65)$ are now evaluated for sume realistic parameters.

Peak torque $=100$ in-02

Load Inertia $=50$ in-oz-sec ${ }^{2}$

Ball screw equivalent $\mathrm{N}=500$

Rotor plus ball screw inertia $=0.01$ in-oz-sec ${ }^{2}$

When these parameters are inserted into Eq. $(12-65), \alpha=10^{4}$ rad; $\mathrm{sec}^{2}$. For a step of 1.8 degrees or 0.031 radian, Eq. (12-64) gives a step time of $3.5 \mathrm{msecs}$. The pulse rate for a rigid load cannot exceed 285 gulses isec.

However, the load is not rigid such that the stepper motor and luad may get out of synchronization resulting in an instability. In order to demonstrate the operation of a stepper motor, a simulation was generated. The simulation program listing is given in Appendix M. The time responses shown are of the block diagram presented in Figure 12-12.

The possibility of the stepper inotor falling out of synchronization with the load is illustrated in Figure 12-13.

ia order to prevent the stepper motor from getting out of synchronization with the load, the pulse rate must be controlled. The pulse raie may be controlled by making it a function of the load damping. When the load receives a position command, the error response in the time domain is

$$
\epsilon(t)=e^{-\delta \omega t} \sin \omega t
$$

where

$\delta=$ Load damping ratio

$\omega=\sqrt{\frac{k_{c}}{J_{l}}}$, load natural f:sequency

This sesponse is shown in Figures $12-14$ through 12.16 for damping ratios of $0.01,0.1$, and 0.5 . It is seen from Eq. $(12-66)$ and the referenced figures that the nominal response follows the envelope of the damped sinusoid. 


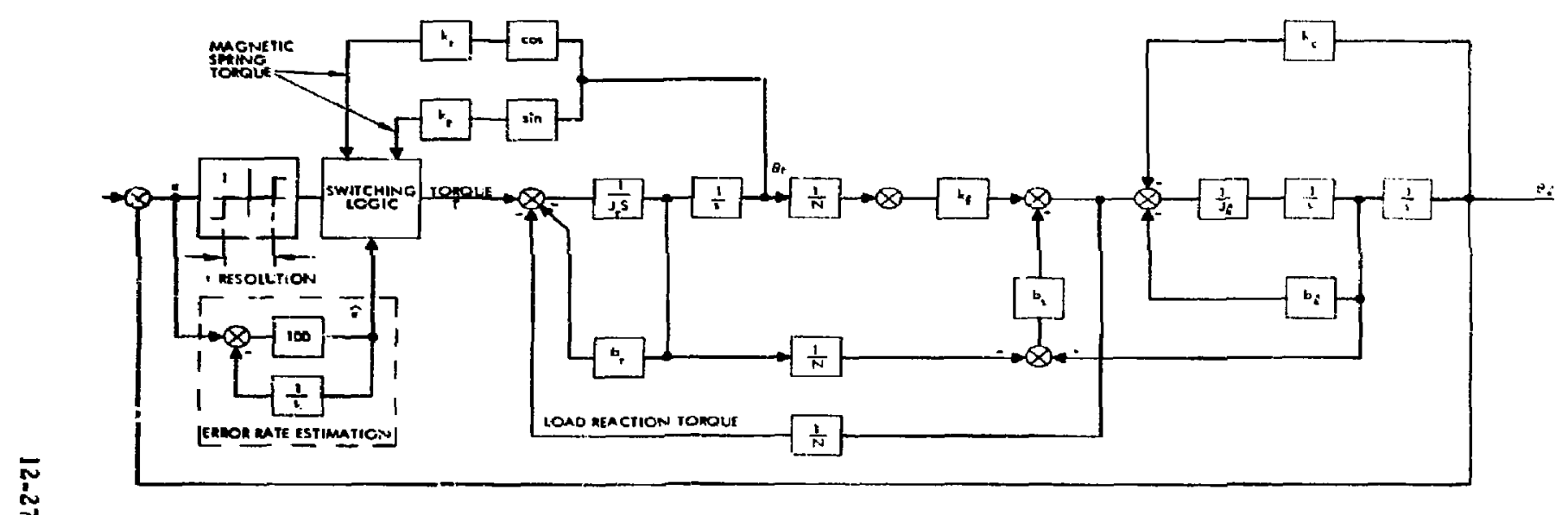

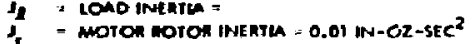
milt scmermatio = 300

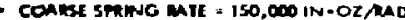

= Fine smanc maie a lso in -0zano

- smano onmpras - 0

$=$ Lond dampantic

$=z_{0}\left(J_{1} h_{e}\right)^{1 / 2} \mathrm{wn}-0 \mathrm{Oz} / \mathrm{mad} / \mathrm{sic}$

- Danpine raito 0.5-0.01
}

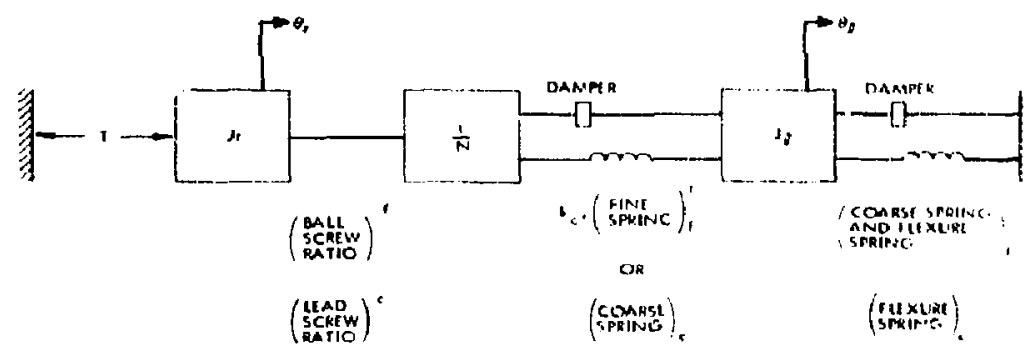

Figure 12-12. Block diagram of simulation 


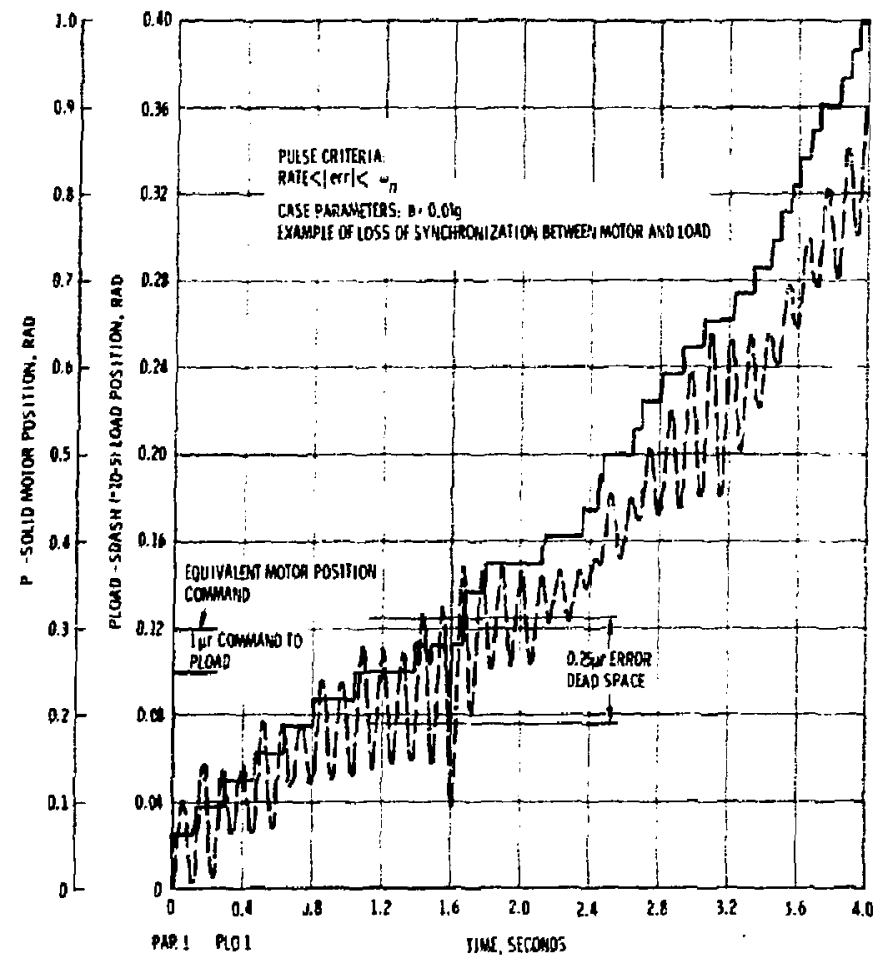

Fisure 12-13. Motor pusition and load position as a function of time 


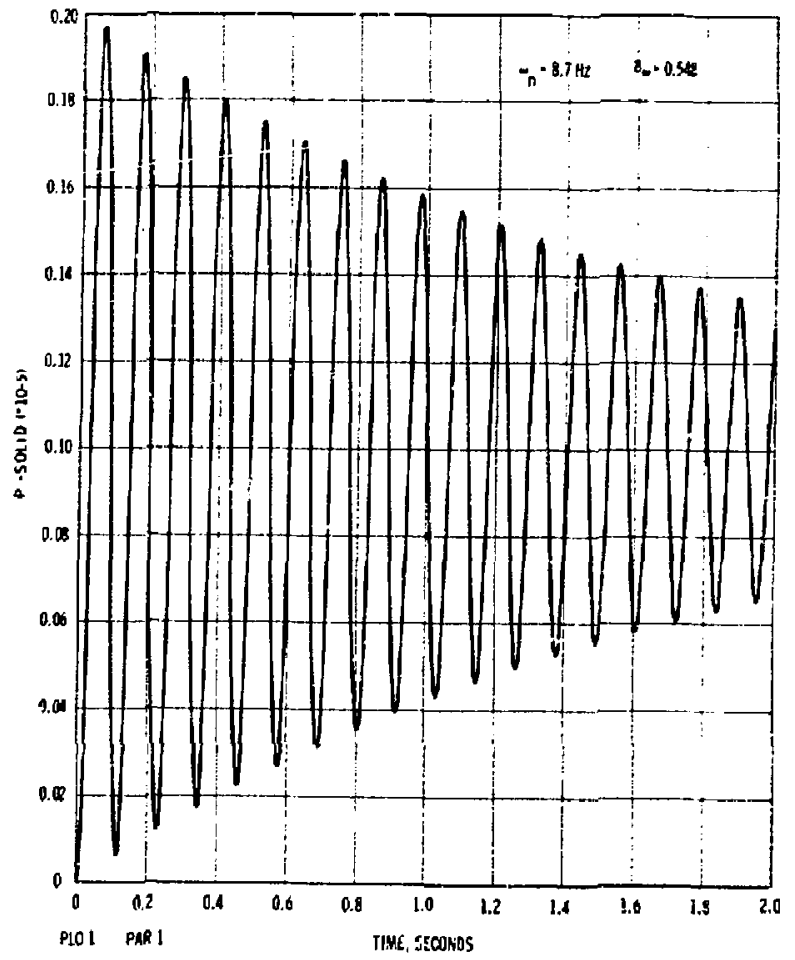

Figure 12-14. Position as a function of time for load with damping ratio of 0,01 and $l \mu \mathrm{rad}$ command 


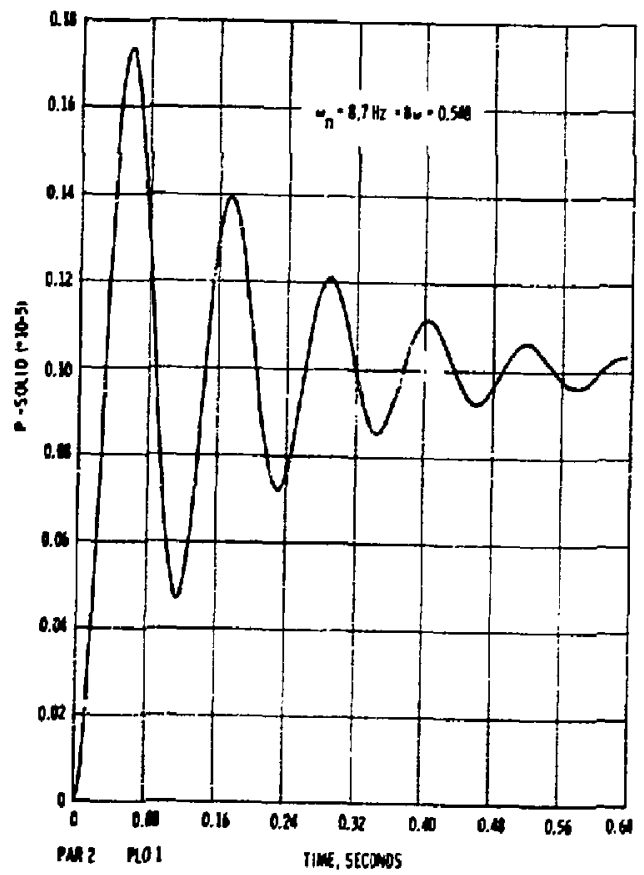

Figure 12-15. Position as a function of sime for load with damping of 0,1 and $1 \mu \mathrm{raa}$ command 


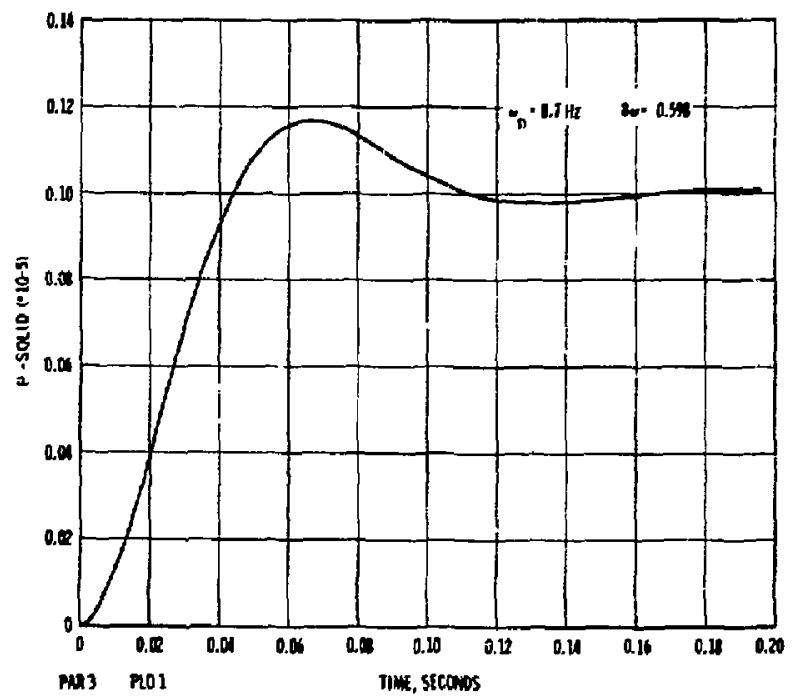

Figure 12-16. Position as a function of time for load with damping ratio of 0.5 and $l \mu \mathrm{rad}$ command

$$
\epsilon(t)=e^{-\delta \omega t}
$$

Therefore, to insure absolute stability the load should not receive anocher position command until it has damped out to where the position error is within the resolution of the system. This stability may be obtained by basically delaying the pulse rate in an open loop manner or controlling the pulse rate as a function of error magnitude, sign, and rate.

The delay of a pulse in an open loop manner is examined first. The servo loop of interest is shown in Figure 12-17.

The closed loop response is given by

$$
\frac{G(s)}{1+G(s)}=\frac{k(-T s+1)}{\left[+\frac{\left.\left[s^{2} ; \omega^{2}\right)+2 \delta(s / \omega)+1\right](T s+1)}{k(-T s+1)}\right.}
$$




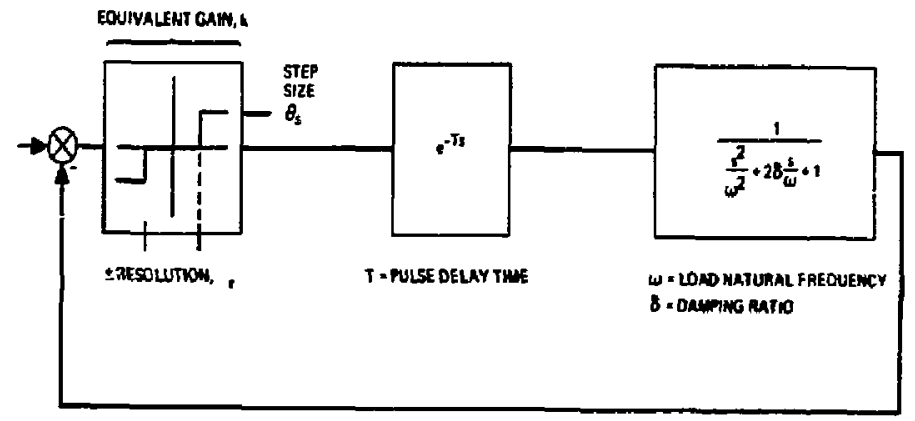

Figure 12-17. Block diagram of servo used to determine pulse delay requirement

The time delay, $T$, in Figure 2-17 is represented by means of a first order Padé approximation in Eq. (12-68). The nonlinear resolution element is represented by an equivalent gain. The stability of the loop is determined by the suots of the characteristic Eq. (12-68).

$$
\Delta(3)=\left(\frac{s^{2}}{\omega^{2}}+2 \delta \frac{s}{\omega}+1\right)(T s+1)+k(-T s+1)
$$

The Routh-Hurwitz stability criteria is applied to Eq. (12-69) for a ga in given by

$$
\begin{gathered}
k=\frac{\theta g}{c}=0.4 \\
\frac{(2 \delta \omega T+1)\left[(1-v) T \omega^{2}+2 \delta \omega\right]>\left(\omega^{2}+k \omega^{2}\right) T}{\omega(\omega T)^{2}(1-k)+(1-k) \omega T+4 \delta^{2} 2 \delta}>1+k \\
\frac{1.2 \delta(\omega T)^{2}+0.6 \omega T+4 \delta^{2}+2 \delta}{\omega T}>1.4
\end{gathered}
$$




$$
1.2 \delta(\omega T)^{2}-0.8 \omega T+4 \delta^{2}+2 \delta>0
$$

For example, if $\delta=0.1$

$$
\omega T=6.36
$$

Therefore, for a $10 \mathrm{~Hz}$ natural frequency at the load the maximum pulse rate is 10 pulses per second. Although the system is absolutely stable the response time is very long due to the fact that we are basically commanding the system open loop.

The possibility of controlling the pulse sate as a function of estimated error sate, magnitude and sign was invistigated by means of simulation. The pulse criteria is given by

$$
\begin{array}{ll}
\text { 1. } & |\dot{\epsilon}|<\delta \omega|\epsilon| \\
\text { 2. } & \text { iो }<0 \\
\text { 3. } & \epsilon>\epsilon
\end{array}
$$

The logic and error rate estimation may be implemented with a couple of operation amplifiers and some diodes.

The response of the system is shown in Figures $12-18$ through 12-20 which illustrate the effect of damping on the response when the pulse rate is controlled. Figures 12-21 and 12-22 illustrate the difference in response between a fixed pulse rate and a controlled pulse rate. Figure 12-23 shows the effect of changing the load resonance from $8.5 \mathrm{~Hz}$ to $10 \mathrm{~Hz}$ and also the load rate is brought up to the maximum pulse rate.

\section{Concluaion to Synthesis}

It has been shown that pointing and holding down to a resolution of $0.25 \mu \mathrm{rad}$ is possible with a conventional servo motor and flexure system if the inertia between the friction source and load is large enough. If the 


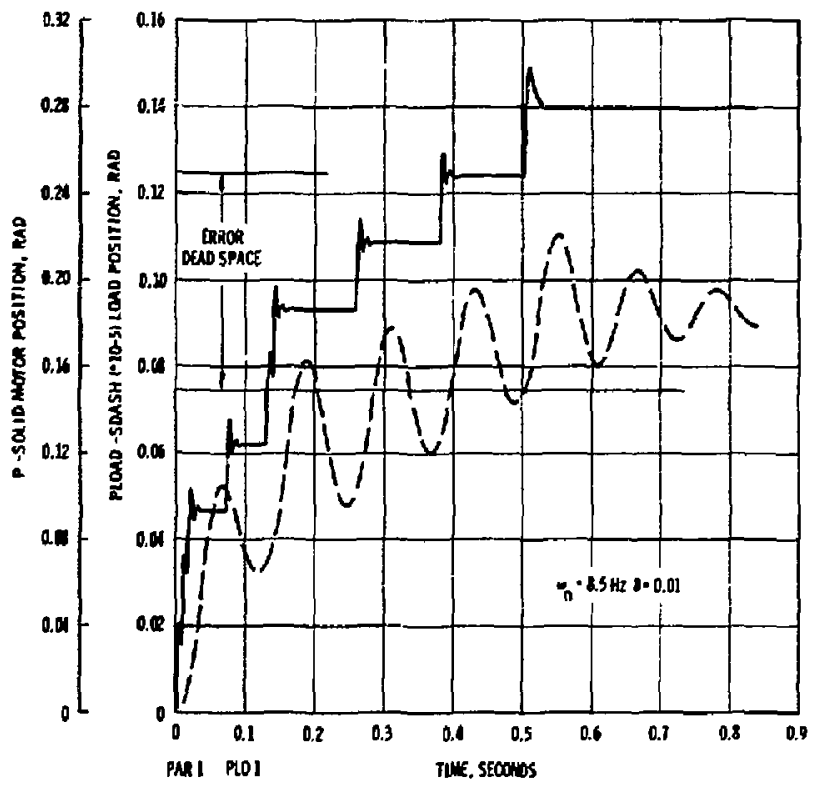

Figure 12-18. Motor and load position as a function of time for controlled pulse rate 


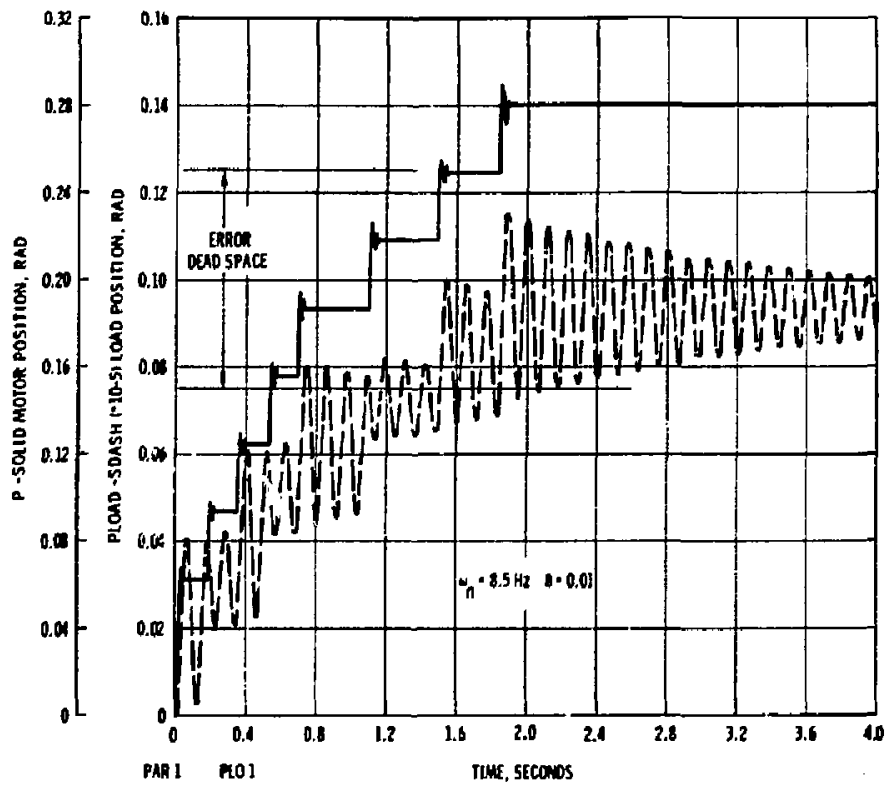

Figure 12-19, Motor and load position as a function of time for controlled pulse rate 


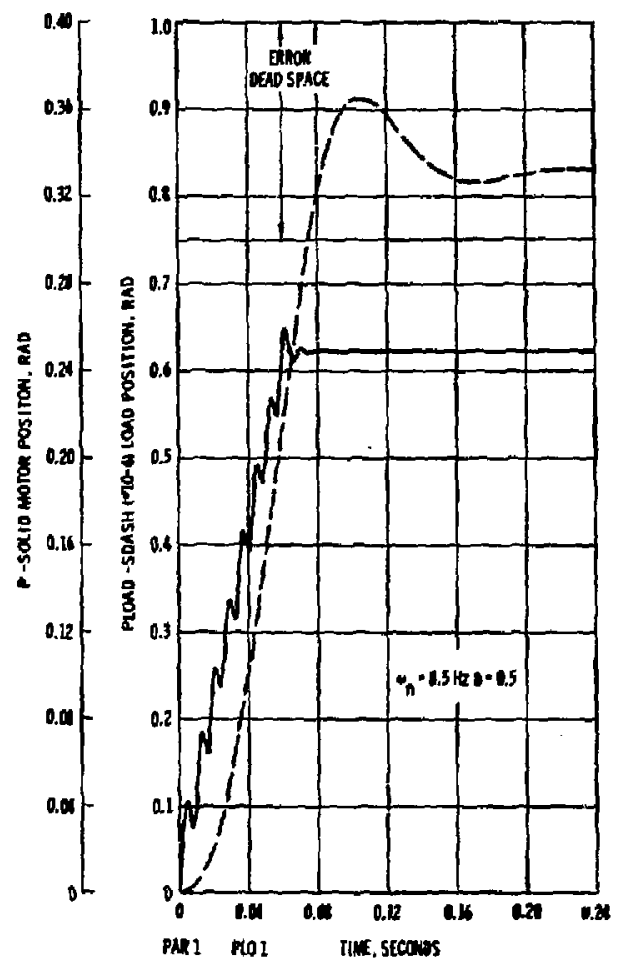

Figure 12-20. Motor and load position as a function of time for controlled pulse rate 


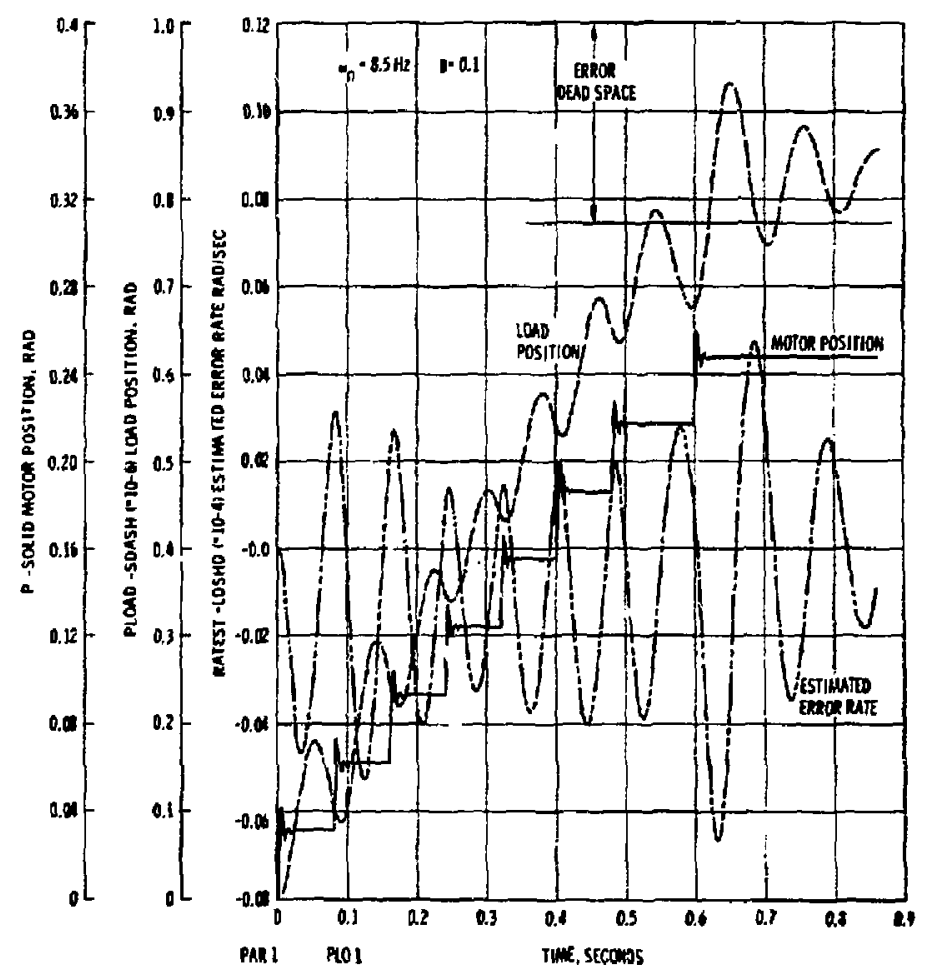

Figure 12-21. Motor position and load position as a function of time for fixed pulse rate of bw 


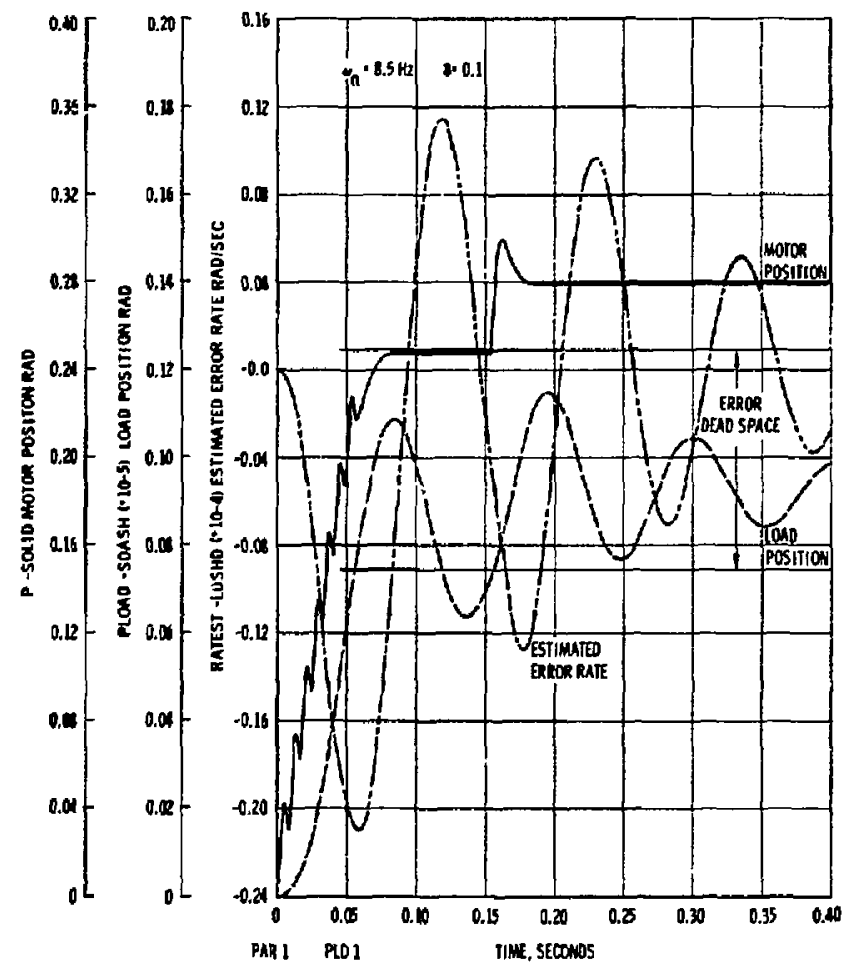

Figure 12-22. Motor position and load position as a function of time for controlled pulse rate 


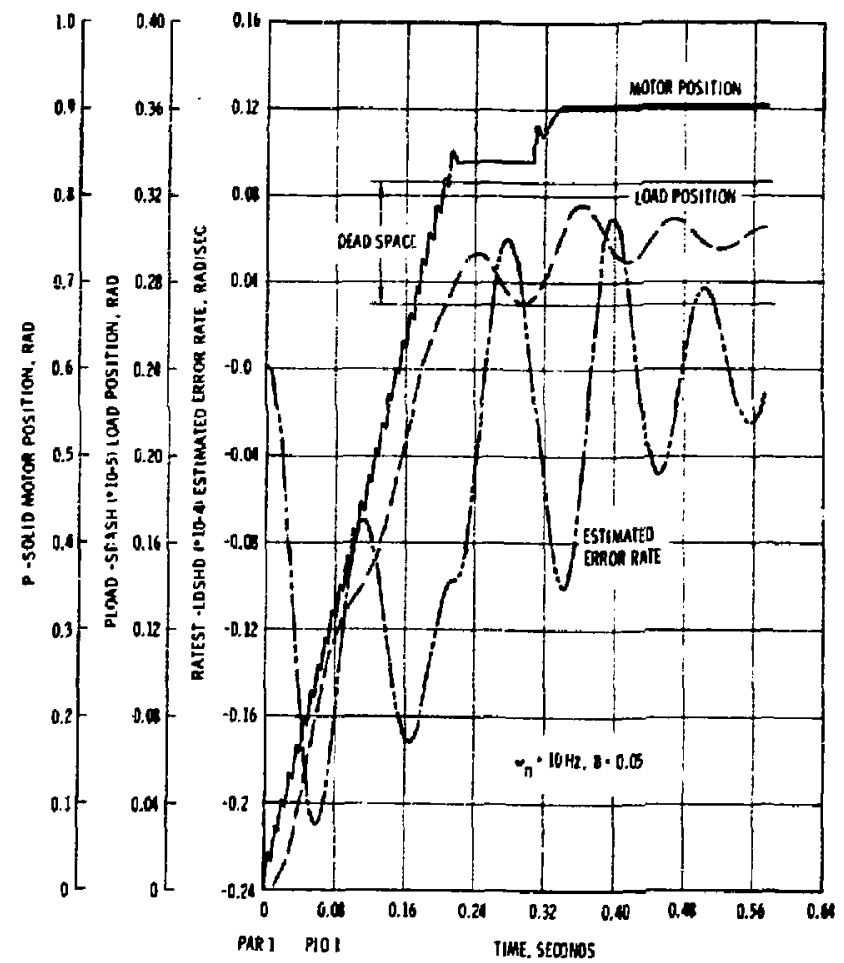

Figure 12-23. Motor and load position as a function of time for $\omega_{n}=10 \mathrm{~Hz}$, $\delta=0.05$

inertia is not large enough, a step motor should be used. The step motor attenuates the friction torque error at the motor through its magnetic spring rate. By controlling the pulse rate of the motor a linear response is achieved which has a time constant of $\delta w$ until maximum pulse rate at the motor is achieved. 


\section{SPRING RATIO SERVO ANALYSIS}

The synthesis in the previous section indicated that the pointing and holding with power off could be achieved with either a motion reduclion by means of levers and a servo motor or a motion reduction by means of springs and a stepper motor. The spring motion reduction was selected on the basis of packaging. This section consists of an analysis of the particular configuration selected. The analysis examines the static stability urfer power off conditions and the transient stability of stepping the motor and load to the desired pesition.

Mechanical analysis indicated that the total range cannot be covered by one spring and one rotary-to-linear motion conve rter. Two drives are used: a coarse drive to position the load to within $25 \mu \mathrm{rad}$ and a fine drive to position the load to within 0.25 prad. A mechanical diagram that is applicable to control analysis is presented in Figure 12-24. The coarse orive utilizes a lead screw to insure that reaction torque from the fine drive will not cause coarse drive displacement. The fine drive utilizes a ball screw to minimize friction.

\section{Calculation of Control Parameters}

The mechanical and electrical parameters of the load and motor which are germane to the servo analysis are now determined.

\section{Load Parameters}

The natural frequency of the load and flexure, $\omega_{n f}$, is about $4 \mathrm{~Hz}$. The load inertia, $J_{l}$, is about 50 in-oz-sec ${ }^{2}$. The flexure spring rate is then given by

$$
k_{\mathrm{cf}}=\omega_{\mathrm{nf}}^{2} \mathrm{~J}_{l}=(4)^{2}(6.28)^{2}(50) \approx 3.2 \times 10^{4} \mathrm{in-0z} / \mathrm{rad}
$$




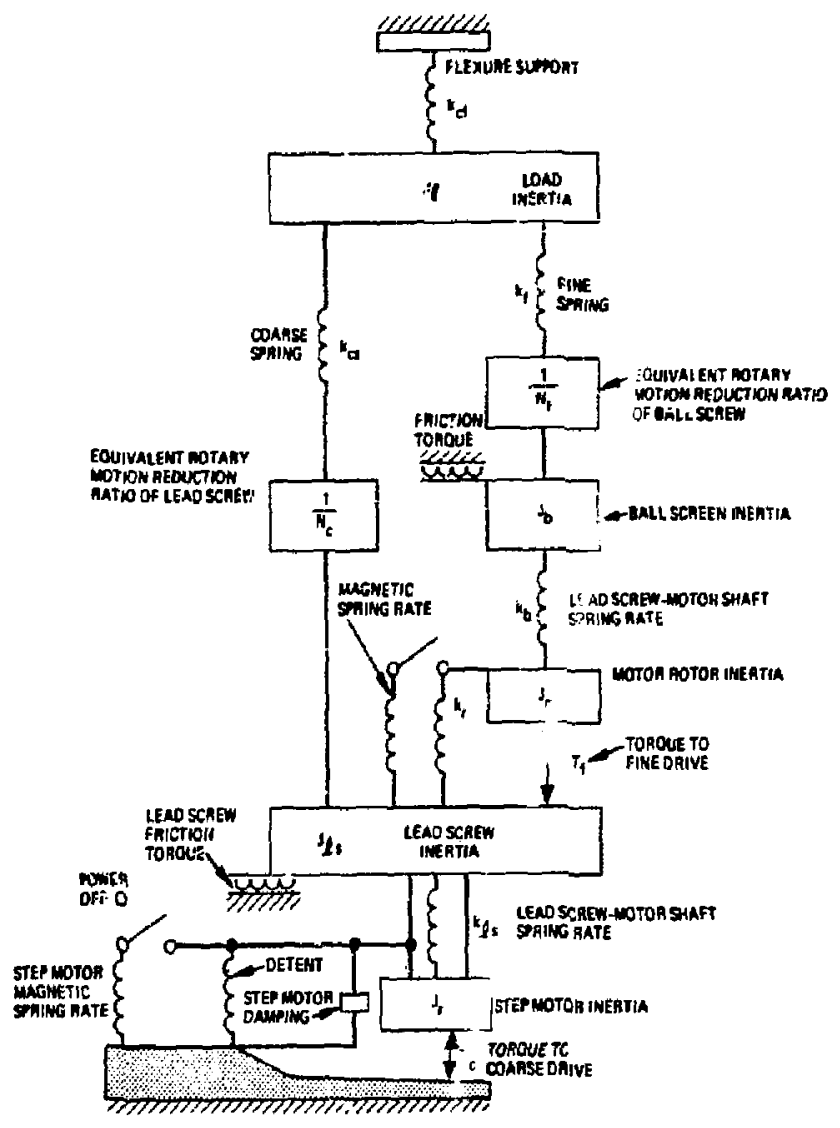

Figure 12-24, Mechanical diagram illustrating control parameters

\section{Coarse Spring Rate}

The natural frequency of the load, hexure, and coarse spring, $\omega_{n c}$ ' is about $10 \mathrm{~Hz}$. The coarse spring rate is then given by

$$
\begin{aligned}
k_{c s}=w_{n c}^{2} J_{l}-k_{c f} & =(10)^{2}(6.28)^{2}(50)-3.2 \times 10^{4} \\
& \approx 16.8 \times 10^{4} \mathrm{inmoz} / \mathrm{rad}
\end{aligned}
$$




\section{Composite Carse Spring Rate}

The spring rate that fint epring force is balanced with is the sum of the flexure spring, $k_{c f}$ and coisse spring rate, $k_{c s}$.

$$
k_{c}=k_{c i}+k_{c s}=20 \times 10^{4} \mathrm{i}:-0 z / \mathrm{rad}
$$

\section{Lead Screw_Prameters}

The inertia of the lead screw, $j_{\ell g^{\prime}}$ with reference to the input is about 0.005 in-oz-sec ${ }^{2}$. The equivalent angular spring rate, $k_{l s}$, of the lead screw and motor shaft is about $10^{7}$ in-oz/rad when measured at the input. The equivalent rotary moticn seduction, $\mathbb{N}_{c}$, of the lead screw is 1000 , a: given by

$$
\left.N_{c}=(\text { lead, in } / \mathrm{rad})^{-1} \text { (Moment arm at load }\right)=1000
$$

The inertia of the fine stspper motor when reflected back through the lead screw ratio is negligible. The friction torque of the lead screw at the input is 10 in 02 .

\section{Ball Screw Parameters}

The inertia of the ball screw, $J_{b}$, is about 0.005 in-oz-sec ${ }^{2}$ when referenced to the input. The spring rate of the ball screw is about $1.5 \times$ $10^{6}$ in-ozirad at the input. The friction toriue at the innut to the ball screw is 1 in-oz.

\section{Stepper Motor Parameters}

The stepper motor is selected on the basis of providing sufficient electromagnetic torque to minimize the effect of friction in the lead screw and ball serew and enough detent torque to provide holding in the fine drive. The motor tentatively selected as a sufficient torque source and minimum step of 1.8 degrees is a size 23 motor from Computer Devices, Model No. 23D-6309. The motor has 3 peak torque of 100 inmoz and a detent torque of $10 \mathrm{in-0z}$. The linear spring rate is illustrated in Figure 12-25. 


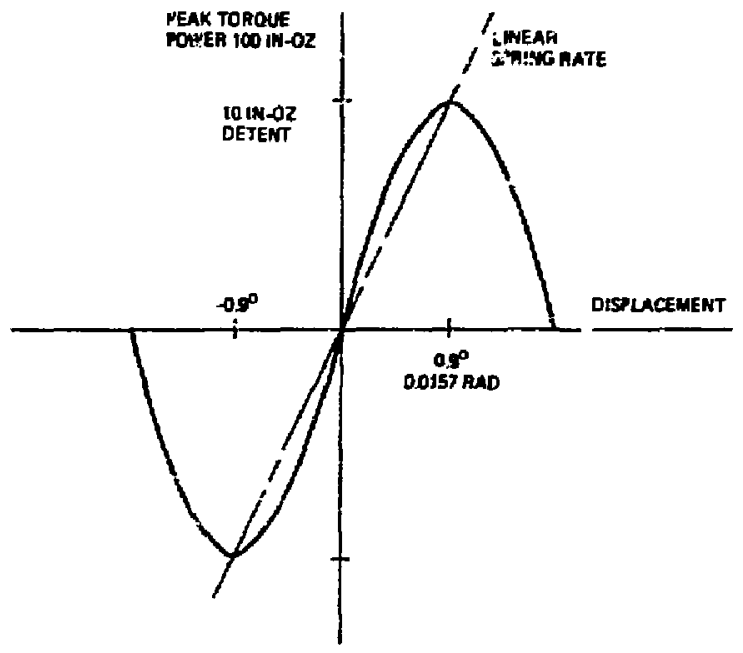

Figure 12-25. Linearized magnetic and electromagnetic spring rate

The linear electromagnetic and magnetic spring rates are given by Eq. (12-82) and (12-83), respectively.

$$
\begin{aligned}
& k_{r}=\frac{(2)(\text { Peak Torque) }}{\text { (Step Displacement })}=\frac{(2)(100 \mathrm{in-0a})}{(0.0314 \mathrm{rad})}=6380 \mathrm{in-oz} / \mathrm{rad} \\
& k_{\mathbf{r d}}=\frac{(2)(\text { Detert Torque })}{(\text { Step Dispiacement })}=\frac{(2)(10 \mathrm{in-oz})}{(0.0314 \mathrm{rad})}=638 \mathrm{in-0z} \mathrm{rad}
\end{aligned}
$$

The linear motor damping is approximated from the spetd torque curve shown in Figure 12-26.

For parameters approximated from Figure 12-26, the linear damping is given by

$$
\begin{aligned}
& b_{r}=\frac{120 \text { ineog }}{(1200 \text { pulses } / \text { seck0.0314 rad/pulse })} \\
& =3.2 \mathrm{in}-0 \mathrm{z} / \mathrm{rad} / \mathrm{sec}
\end{aligned}
$$




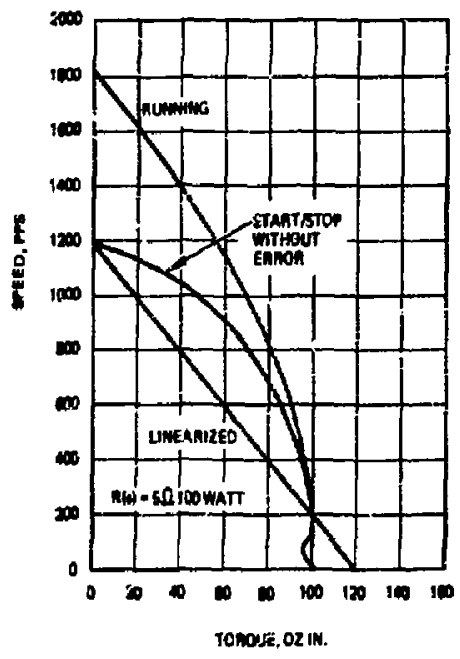

Figure 12-26. Speed torque curve

The motor bas an inertia of 0.005 in-oz- $\sec ^{2}$ which if rigidly coupled to the ball or lead screw results in an inertia of 0.01 inuoz-sec 2 . The linear motor transfer function for torque in to position out in is given by

$$
\begin{aligned}
\frac{\theta_{r}}{T_{r}} & =\frac{1}{J_{r} s^{2}+b_{r} s+k_{r}}=\frac{1}{0.01 s^{2}+3.2 s+6380} \text { \{electro magnetic) } \\
& =\frac{1 / 6380}{\left(s^{2} / 796^{2}\right)+(2)(0.2)(s / 796)+1} \\
& =\frac{1}{0.01 s^{2}+3.2 s+638} \text { (magnetic) } \\
& =\frac{1 / 638}{\left(s^{2} / 252^{2}\right)+(2)(0.65)(s / 252)+i} \text { (nagnetic) }
\end{aligned}
$$


It may be noted that the natural frequency, $\omega_{n}=796 \mathrm{rad} / \mathrm{sec}$, is quite a bit higher than the load resonant frequency. Because the motor and load coupling is cumpliant, a stability problem exists due to this large difference between load and motor natural frequencies.

The abrye electromechanical parameters are summarized in Table 12-1. Static Siability of Position Servo

The ability of the positioning mechanism to maintain the commanded position with prwer off is now investigated. The coarse gerro must meet two requirements. It must have

- Enough friction at the load to maintain the position at the travel extremes.

- Enough electromagnetic friction torque attenuation to meet the coarse positioning requirement of $25 \mu \mathrm{rat}$.

The maximum holding torque of the coarse servo at the load is given by the product of the maximum travel and the flexure spring rate.

$$
\begin{aligned}
\mathrm{T}_{\mathrm{f} \text { (at motor) }} & =\frac{\left(\begin{array}{c}
\text { Maximum } \\
\text { Displacement }
\end{array}\right)\left(\begin{array}{c}
\text { Fexure } \\
\text { Spring Rate }
\end{array}\right)+\left(\begin{array}{l}
\text { Reaction Torçue } \\
\text { from Fine Drive }
\end{array}\right)}{\text { (Lead Screw Ratio) }} \\
& =\frac{(0.010 \mathrm{rad})\left(3.2 \times 10^{4} \mathrm{in-0z} / \mathrm{rad}\right)+(200 \mathrm{in}-0 \mathrm{z})}{(1000)} \\
& =0.4 \mathrm{in-0z}<\mathrm{T}_{\mathrm{fcl}}=10 \mathrm{in-0z}
\end{aligned}
$$

The static friction error attenuation is obtained from Equation (12-59) of the Systhesis section. It is given approximately by

$$
\theta_{\ell}=\frac{\text { (Static Friction Torque)(Cuarse Spring Rate) }}{\text { (Electromagnetic Spring Rate)(Lead Screw Ratio) }\left(\begin{array}{c}
\text { Rate + Flexure } \\
\text { Spring Rate }
\end{array}\right)(12-86)}
$$

$$
=\frac{(20 \mathrm{in}-0 \mathrm{z})\left(16.8 \times 10^{4} \mathrm{in}-0 \mathrm{z} / \mathrm{rad}\right)}{(6380 \mathrm{in}-0 \mathrm{z} / \mathrm{rad})(1000)\left(20 \times 10^{4}\right)}=2.65 \mu \mathrm{rad}<25 \mu \mathrm{rad}
$$


Table 12-1. Summary of electro-mechanical parameters

\begin{tabular}{|c|c|c|c|}
\hline Parzmeter & Symbol. & Yalue & Units \\
\hline $\begin{array}{l}\text { Load Inertia } \\
\text { Motor Rotor Inertia } \\
\text { Ball Screw Input Inertia } \\
\text { Lead Screw Input Inertia }\end{array}$ & $\begin{array}{l}J_{l} \\
J_{x} \\
J_{b} \\
J_{l s}\end{array}$ & $\begin{array}{l}50 \\
0.005 \\
0.005 \\
0.905\end{array}$ & in-oz-sec ${ }^{2}$ \\
\hline $\begin{array}{l}\text { Eloctromagnetic Motor } \\
\text { Dampisg } \\
\text { Magnetic Alotor Damping } \\
\text { (Detent) } \\
\text { Laad Flexurc Damping }\end{array}$ & $\begin{array}{l}B_{I} \\
B_{r d} \\
B_{l}\end{array}$ & 3.2 & in-oz/rad/sec \\
\hline $\begin{array}{l}\text { Load-Flexuse, } \omega_{n} \\
\text { Load-Coarse Spring, } w_{n} \\
\text { Motor, Electromagnetic, } w_{n} \\
\text { Molos, Magmetic, } w_{n}\end{array}$ & $\begin{array}{l}\omega_{\mathrm{nf}} \\
\omega_{\mathrm{ne}} \\
\omega_{\mathrm{nm}} \\
\omega_{\mathrm{nind}}\end{array}$ & $\begin{array}{l}=4 \\
=10 \\
125 \\
40 \\
\end{array}$ & Hertz \\
\hline $\begin{array}{l}\text { Flexure Spring } \\
\text { Coarse Spring Rate } \\
\text { Fine Spring Rate } \\
\text { Ball Screw Liput Spring Rate } \\
\text { Lead Screw input Spring Rate } \\
\text { Electromagnetic Spring Hate } \\
\text { Magnetic Speing Ratc }\end{array}$ & $\begin{array}{l}k_{\text {ef }} \\
k_{\text {es }} \\
k_{1} \\
k_{b} \\
k_{i} \\
k_{r} \\
k_{\text {rd }}\end{array}$ & $\begin{array}{l}3.2 \times 10^{4} \\
16.8 \times 10^{4} \\
300 \\
1.5 \times 10^{5} \\
10^{7} \\
6380 \\
638\end{array}$ & jn-cz/rad \\
\hline $\begin{array}{l}\text { Eall Sesew Static/Seulomb } \\
\text { Friction } \\
\text { Lead Serew Static/Coulomb } \\
\text { Erietion }\end{array}$ & $\begin{array}{l}T_{f o b} / T_{f e b} \\
T_{\text {tse }} / T_{\text {fel }}\end{array}$ & $\begin{array}{l}1.010 .5 \\
20 / 10\end{array}$ & (itt-oz)-sign of ratio) \\
\hline $\begin{array}{l}\text { Ball Serew Equivalen! } \\
\text { Motion Ratio } \\
\text { Lead Serew Equivalen! } \\
\text { Motion Ratio }\end{array}$ & $\begin{array}{l}N_{b} \\
N_{l}\end{array}$ & $\begin{array}{l}500 \\
1000\end{array}$ & $\begin{array}{l}-\infty \\
=-\end{array}$ \\
\hline $\begin{array}{l}\text { Maximum Motor Torque } \\
\text { Detent Torque }\end{array}$ & $T_{\text {max }}$ & $\begin{array}{l}100 \\
10\end{array}$ & in $=0 z$ \\
\hline Motor Resistance per phtsc & $R$ & 0.48 & ohms \\
\hline Current per phase & 1 & A. 6 & amps \\
\hline
\end{tabular}


The fine drive static stability is now investigated. The fine drive must provide enough magnetic torque to hold the load at: its extreme displacement of 25 rad. The relationship is given by

$$
\begin{aligned}
& \frac{\left(\begin{array}{c}
\text { Coarse } \\
\text { Increment }
\end{array}\right)\left(\begin{array}{c}
\text { Flexure Spring } \\
\text { Rate }
\end{array}\right)+\left(\begin{array}{c}
\text { Coarse Spring } \\
\text { Rate }
\end{array}\right)}{\text { (Ball Screw Ratio) }}<(0.1)\left(\begin{array}{c}
\text { Peak Magnetic } \\
\text { Torque }
\end{array}\right)(12-88) \\
& \frac{\left(25 \times 10^{-6} \times(16.8+3.2) \times 10^{4} \text { in-oz } /\right. \text { rad }}{500}=0.01 \text { in-0z< }(0.1)(10 \text { in-0z) (12-89) }
\end{aligned}
$$

The maximum static displacement error of the fine drive is due to static friction torque, loar spring torque calculated above, and nominal step-tostep accuracy.

$$
\begin{aligned}
\begin{array}{l}
\text { Error due to Load } \\
\text { isplacement of } 25 \mu \mathrm{rad}
\end{array} & =\frac{(0.01 \text { in-oz })(\text { Magnetic Spring Rate })^{-1}}{(\text { Ball Screw Ratio) }(\text { Spring Ratio })} \\
& =\frac{(0.01 \text { in-oz })(638 \mathrm{in-oz} / \mathrm{rad})^{-1}}{(500)(1000)} \\
& =0.3 \times 10^{-10} \mathrm{rad}
\end{aligned}
$$

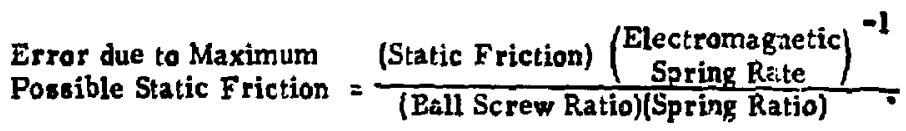
$=\frac{(1 \text { in-oz })(6380 \text { in-0z/rad })^{-1}}{(\text { Ball Screw Ratio)(Spring Ratio) }}$

$=0.3 \times 10^{-9} \mathrm{rad}$

Error due to Step = (Nominal Step Width)(Percent Variation) Accuracy (Ball Screw Ratio)(Spring Ratio)

$$
=\frac{(0.0314)(0.03)}{0.5 \times 10^{6}}=0.2 \times 10^{-8} \mathrm{rad}
$$


The static stability of both the coarse and the fine position mechanisms has been established.

Dynamic Stability of Position Serwo

There are three areas of possible stability problems.

1. Compliance between the stepper motor and load can result in loss of synchronization between the motor and the load and a resultant instability.

2. The centering and pointing gervos are in series such that excessive time lags within the individual sorvo loops may result in an outer loop stability problem.

3. The pointing servo is in a parallel path with the centering servo but operates in opposition to the centering servo. The static gain of the centering servo path is higher than that of the pointing servo such that statically the overall loop is always stable. However, dynamically it appears to be possible for the pointing servo to introduce positive feedback with a resulting instability.

Stability of Stepper Motor and Load

The stepper motor as a servo element is described in Appendix $L$ and in listed referencis. A block diagram of the fine servo is shown in Figure 12-27. The parameters in Figure 12-27 were identified in Table 12-1. The witching criteria consists of applying positive, negative, or zero cuFrent to a winding which is 90 electrical degrees away from the present position. The logic used in simulating the electronic switching is given in Appendix $M$. Ninety electrical degrees corresponds to one mechanical step of 1.8 degrees or 0.03141 rad. For a rigid drive, the derdspace should be at least twice the step size such that zero error may definitely be sensed in any one step. Because of the drive compliance and potential lack of damping in the load, the deadupace was chosen as five times the step size of the load. The step size at the load for the fine drive is $0.1 \mathrm{\mu rad}$. in Figure 12-27 the motor shaft is assumed to be rigidly coupled to the ball screw such that friction torque at the ball screw is reflected into the motor loop. 


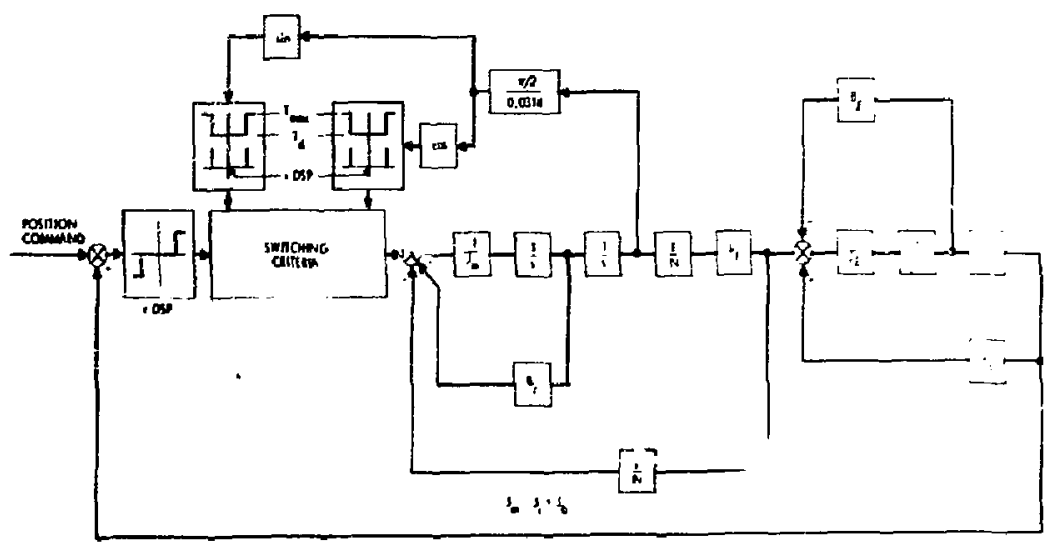

Figure 12-27, Position servo block diagram

The motor bandwidth is over 10 times the resonant frequency of the load and because the ball screw ratio reduces the reaction torque back on the motor, the servo of Figure 12-27 may be approximated by that shown in Figure 12-28. From the servo synthesis equation, it is shown that the delay required for syotem stability is

$$
T=\frac{6.36}{\omega_{n}}, \delta=0.1
$$

where

$$
\begin{aligned}
\omega_{n} & \equiv \text { Load Natural Frequency } \equiv \sqrt{\mathrm{k} / \mathrm{J}_{l}} \\
\delta & \equiv \text { Luad Damping Ratio } \equiv \frac{1}{2} \mathrm{~B} / \sqrt{J_{l} \mathrm{k}_{l}}
\end{aligned}
$$




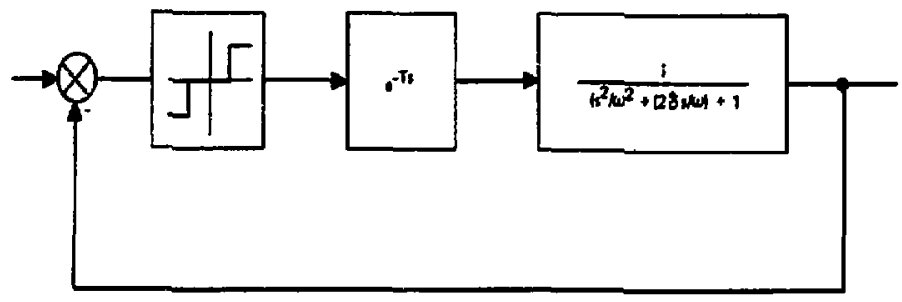

Figure 12-28. Simplified position servo block diagram

From Table 12-1,

$$
\omega_{\mathrm{n}}=62.8 \mathrm{rad} / \mathrm{sec}
$$

such that

$$
T=0.1 \text { for } \delta=0.1
$$

By the use of elastomers or hydraulic bellows, the damping ratio is assumed to be 0.1 , such that the maximum pulse rate is 10 pulses per second. The response over the range is the refore given by adding the response time of the coarse servo and fine servo.

$$
\begin{array}{rlrl}
\text { Total coarse range } & =2 \times 10 \times 10^{3}=2 \times 10^{4} \mu \mathrm{rad} & & (12-98) \\
\text { Total fine range } & =25 \mu \mathrm{rad} & & (12-99) \\
\text { Pulse rate } & =10 \text { pulsesio.c } & (12-100) \\
\text { Coarse pulse amplitude } & =25 \mu \mathrm{rad} & & (12-101) \\
\text { Fine pulse amplitude } & =0.1 \mu \mathrm{rad} & & (12-102) \\
\text { Total time to cover range } & =T_{\mathrm{TR}} &
\end{array}
$$




$$
\begin{aligned}
T_{T R} & =\frac{(\text { Iotal Range) }}{\text { (Pulse Ráte)(Coarse Pulse Amplitude })}+\frac{(\text { Coarse Pulse }(2)}{\left(\begin{array}{c}
\text { Pulse } \\
\text { Rate }
\end{array}\right)\left(\begin{array}{c}
\text { Fine Pulse } \\
\text { Amplitude }
\end{array}\right)}(12-103) \\
& =\frac{2 \times 10^{4} \mu \mathrm{rad}}{(10 \mathrm{pulses} / \mathrm{sec})(25 \mu \mathrm{rad} / \text { pulse })}+\frac{(25 \% \mathrm{rad})(2)}{(10 \mathrm{pulses} / \mathrm{sec})(0.1 \mu \mathrm{rad} / \mathrm{pulse})}(12-104) \\
& =80 \mathrm{sec}+50 \mathrm{sec}=130 \mathrm{sec}
\end{aligned}
$$

If a uniform distribution of target position over range is assumed, then the $1 \sigma$ time to arrive at the target is $130 / \sigma=130 / \sqrt{12}=38$ seconds, if the total range is assumed to cover $\pm 3 \sigma$ on a normal distribution and the target is uniformly distributed over the fine range, the 10 time taken to reach the target is 33 seconds.

By varying the pulse rate with the error rate and error as described in the section on servo synthesis, the travel time may be reduced to the extent that the error follows the envelope of the damped sinusoid.

Design parameters have now been estabiished to insure system stability and a response time based upon those parameters is shown in Figure 12-29.

\section{Coupling Stability}

The coupling between the centering mirror and the pointing mirror is now investigated. The control loops are shown in Figure 12-30 From Figure 12-30 it may be seen that without the dotted path of the centering servo error feed forward signal, the centering and pointing serve dy amics a re directly in series. It may easily be seen from Figure 12-31 and Eq. (12-106) and (12-107), the benefit of the feed forward signal

$$
\begin{aligned}
\frac{Q}{C} & =R+\epsilon \\
& =\frac{G(s !}{1+G(s)}+\frac{1}{1+G(s)}=1
\end{aligned}
$$




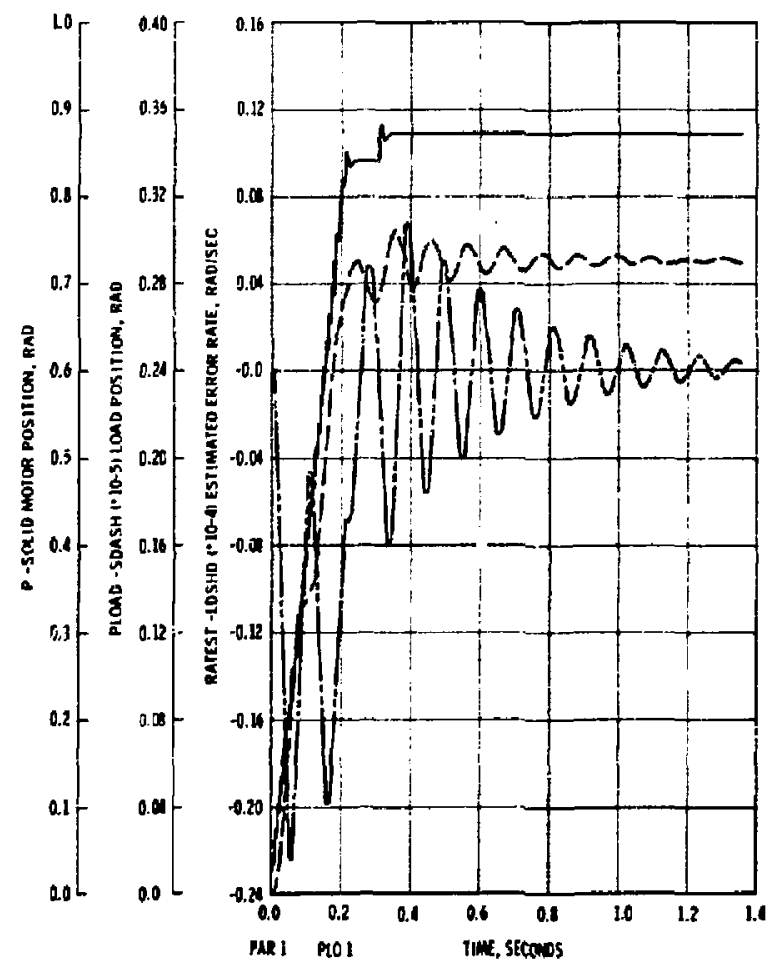

Figure 12-29, Motor position, load position, and estimated load rate for $3 \mu \mathrm{rad}$ command, $\pm 0.25 \mu \mathrm{rad}$ deadspace, 0.1 load damping, and a maximum pulse rate of 125 pulses per aecond 


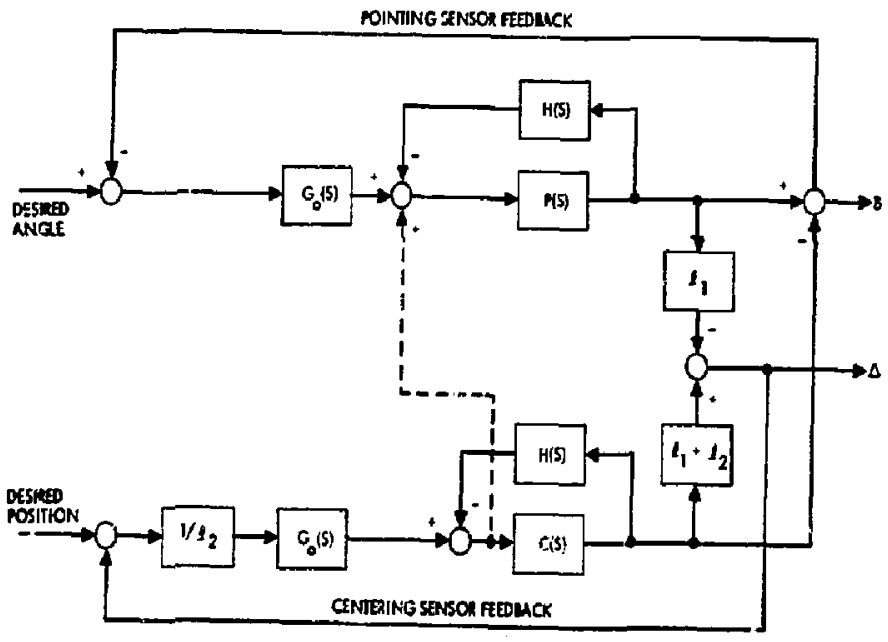

Figure 12-30. Automatic control of eentering and coarse pointing

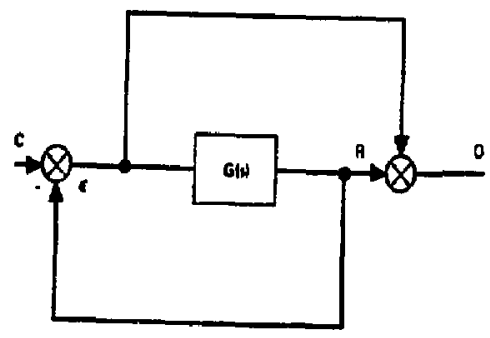

Figure 12-31. Feed forward of error ofgnal 
The possible instability of pointing ses'vo in a positive feedback path is shown in Figure 12-32 for the case of no centering servo dynamics. The parallel paths result in the transfer function given by Eq. (12-10̄8).

$$
1-\frac{k}{\left(s^{2} / \omega^{2}\right)+(2 \delta s / \omega)+1}=\frac{\left[s^{2} /(1-k) \omega^{2}\right]+[2 \delta s((1-k) \omega]+1}{\left(s^{2} / \omega^{2}\right)+(2 \delta s / \omega)+1}(1-k)(12-108)
$$

The numerator of Eq. 12-108 indicates that the resulting zeros are less than the poles. It may be shown with root locus techniques of Figure 12-33 that the loop is unconditionally stable. The integration in Figure 12-32 corresponds to the integrator in the optical loop. Because of the nonlinear nature of the loop, a simulation was also performed of the system shown in Figure 12-30 with the results shown in Figure 12-34. In Figure 12-34, the worst case moment arm example is used with a load damping of 0.10 . A disturbance of 1 mrad for 0.01 second was injected and the loops were stable.

It is concluded that coupling stability is not a problem.

\section{Conclusion}

The analysis of the servo has indicated that there are no stability problems.

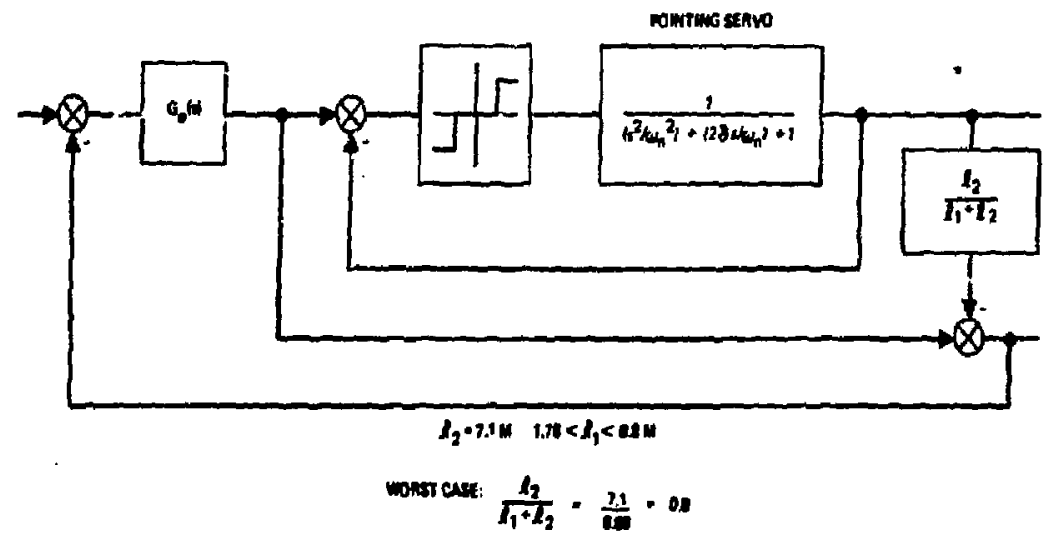

Figure 12-32. Simplified diagram of ponitive feadback path of pointing servo 


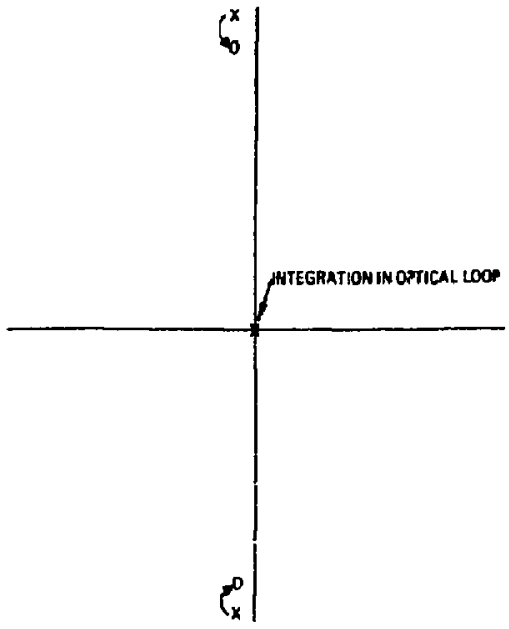

Figure 12-33. Root locus of parallel paths of pointing servo and centering beam

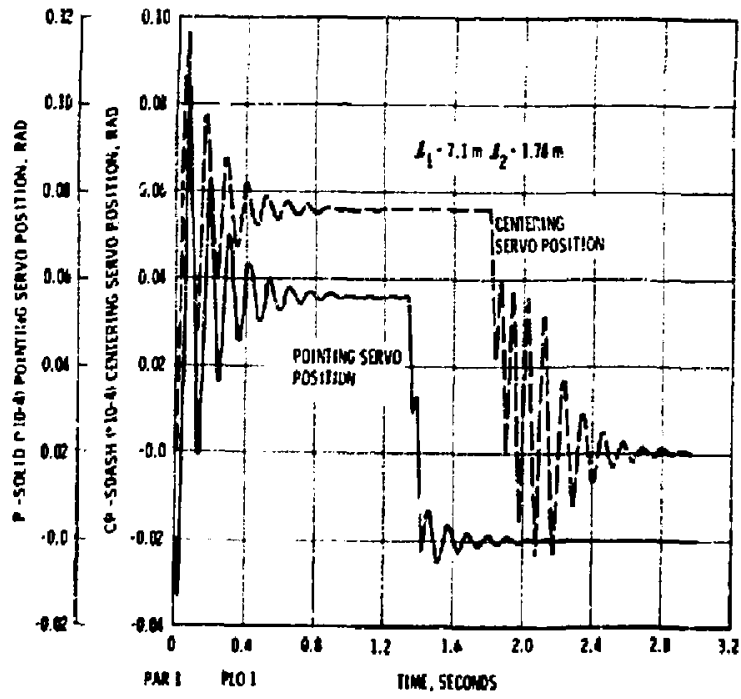

Figure 12-34. Response to a 1 mrad disturbance for 0.01 second into the centering servo and $-1 \mathrm{mrad}$ into the pointing servo. Centering and pointing servo positions versus time 


\section{SER VO HARDWARE DESIGN}

The servo design consists selecting a position sensor which is compatible with producing signals that are proportional to mirror position. The characteristics whicb are of greatest importance are sensitivity and resoiution. Electromagnetic sensors tend to meet these requirements best. Reference 4 provides a good synopsis of the various types of electromagnetic sensors quated below.

On Table 12-2, the relative advantages of each of the major members of this family of transducers is shown. These are four types of transducers. In each category the sypes are ranked relative to one another, number 1 being the best, number 2 next, etc. The numbers are not intended to have an absolute meaning. Some comments on the items in this table follows:

\section{Sensitivity}

The presence of a well defined air gap permits the E-core pickoff and the SCDT to have a greater sensitivity than the LVDT. The reluctance pickoff, varying the small gap length, has the grestest sensitivity of all.

\section{Linearify}

Because of the ease of maintaining a uniform gap length, the SCDT has been rated best. The LVDT can be made just as linear if the core is properly shaped and the coils are carefully wound. The presence of fringins in the movable core makes the maintaining of good linearily in the other two types more difficult.

Nuil Voltage

Since the modt troublesome cause of null voltace is the harmonic content of the output voltage, due to the nonlinearity of the rilagnetic material, the null voltage will be lower on the types having the larger air gap.

\section{Iranaverse Axis Sensitivity}

Both the LVDT and the SCDT are virtually insensitive to displacement in the transverse direction, 28 wes as to angular misalignment of the armature. In contrast, the E-core pickoff is extremely sensitive to these factors. 
Table :2-2. Relative advantages of SCDT

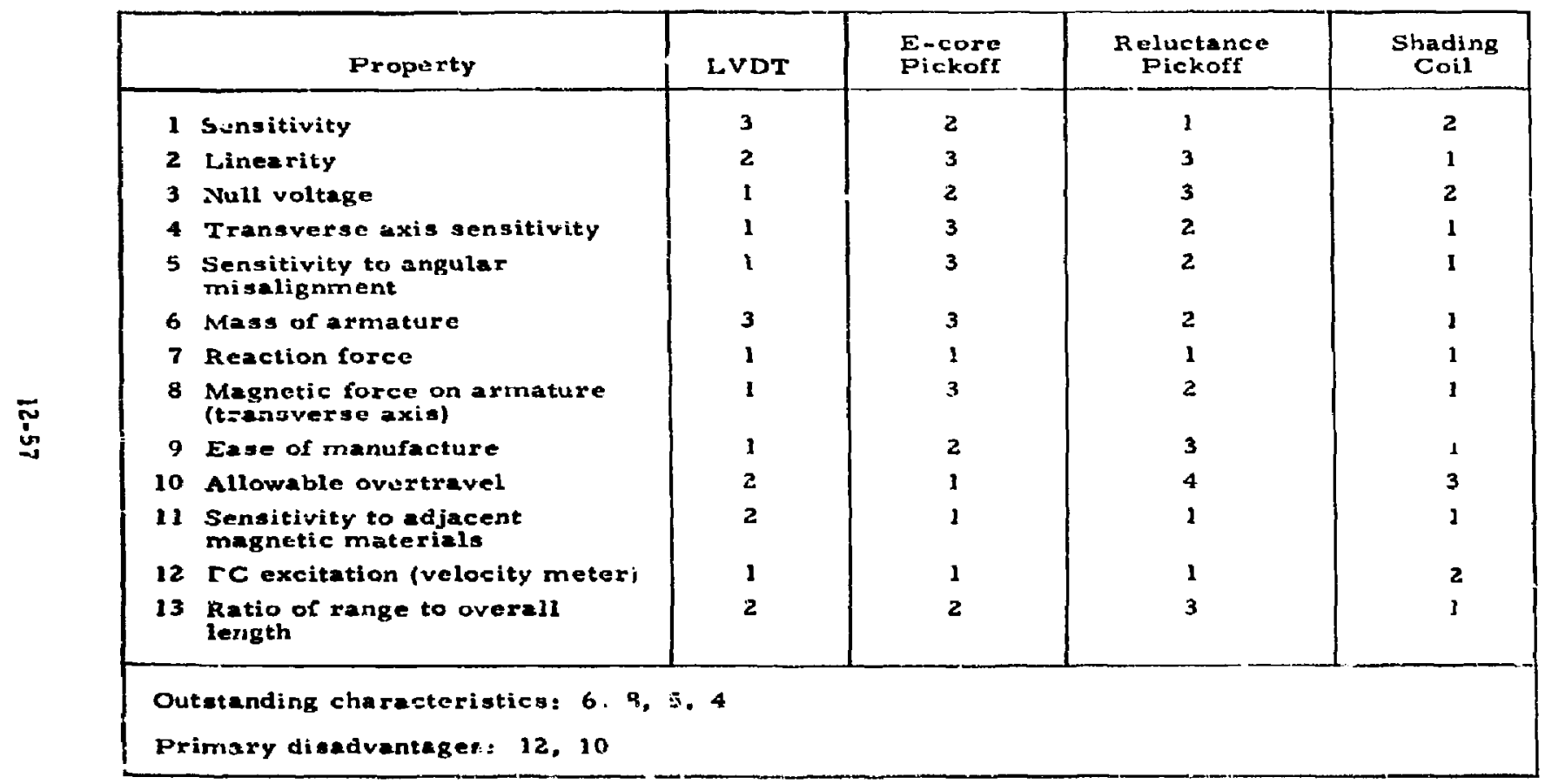




\section{Mass of Armature}

The small mass of the shading coil as opnosed to the much larger mass of the magnetic armature of the other types is an outstanding feature of the SCDT.

\section{Magnetic Force on Armature}

Just as the SCDT and the I.VDT display a low transterse axis sensitivity, so there is also a negligible magnetic force on the armature in the transverse axis. The E-core pickoff, which is the most susceptible to transverse axis motion, experiences the greatest rnagnetic force in the transverse direction.

Ease of Manufacture

Although the expense of manufacturing the components of each of these derices is ruaghly the same, the much more critical alignment of the E-core pickoff and the reluctance pickoii makes these types more difficult to assemble and align.

\section{Allowable Overtravel}

The E-core pickoff permits unlimited overtravel in either direction. The LVDT permits unlimited overtravel in one direciion and a large amount in the other direction. The SCDI permits limited overtravel in either direction. The relu:tance pickoff permits no overtravel.

\section{Excitation}

All of these transducers except the SCDT can be designed to use permanent magnetic excitation in order to measure velocity instead of displacement. Because of the transformer coupling between the shading coil and the output winding, this is not possible in the SCDT.

\section{Applicability}

The applicability profile which can be seen in Table 12-2 would indicate that the SCDT is ideally suited to applications requiring moderately high sensitivity, low transverse sensitivity, low magnetic forces, and especially smal? amature mass. On the other hand, it carnot be used as a velocity meter or in applications involving large overtravel. 
Tentatively, a LVDT witi a 0.05 -inch range is selected. The LVDT output signal is full wave rectified and filtered such that the re is some ripple of the carrier wave on the DC output. This ripple, if not eliminated by the differential amplifier, may be a problem in estimating the rate error. Although the LVDT has infinite resolution, the electronic that processes the signal introduces noise with the result that the meaningful resolvtion out of the LVDT is abjui $10 \mu \mathrm{rad}$ when all the noise sources are added vectorially. The IFDT therefore can only be used to position the coarse drive and fine drive to the base of the fine spring.

A hardware block diagram is shown in Figure 12-35. The tentative LVDT selected has a $6 \mathrm{~K}$ ohm output impedance. Th e signals therefo ve are sent to buffer amplifiers before they are sumned up in a differential cmplifier. There is a gain ircrease such that a diode breakdown voltage $c$ rresponds to $25 \mu \mathrm{rad}$ in that coarse drive and $250 \mu \mathrm{rad}$ in the fine drive rela: ve to the base of the fine spring. A spring ratio of 1000 is assumed.

The error rate estimation is performed with a $16 \mathrm{~Hz}$ loop in order to minimize noise amplification. The deadspace error, product of error and error rates, and comparison of error rate and error are all summed into an AND gate. The fine servo requires one more signal that centers the fine drive when the coarse drive is in motion. 

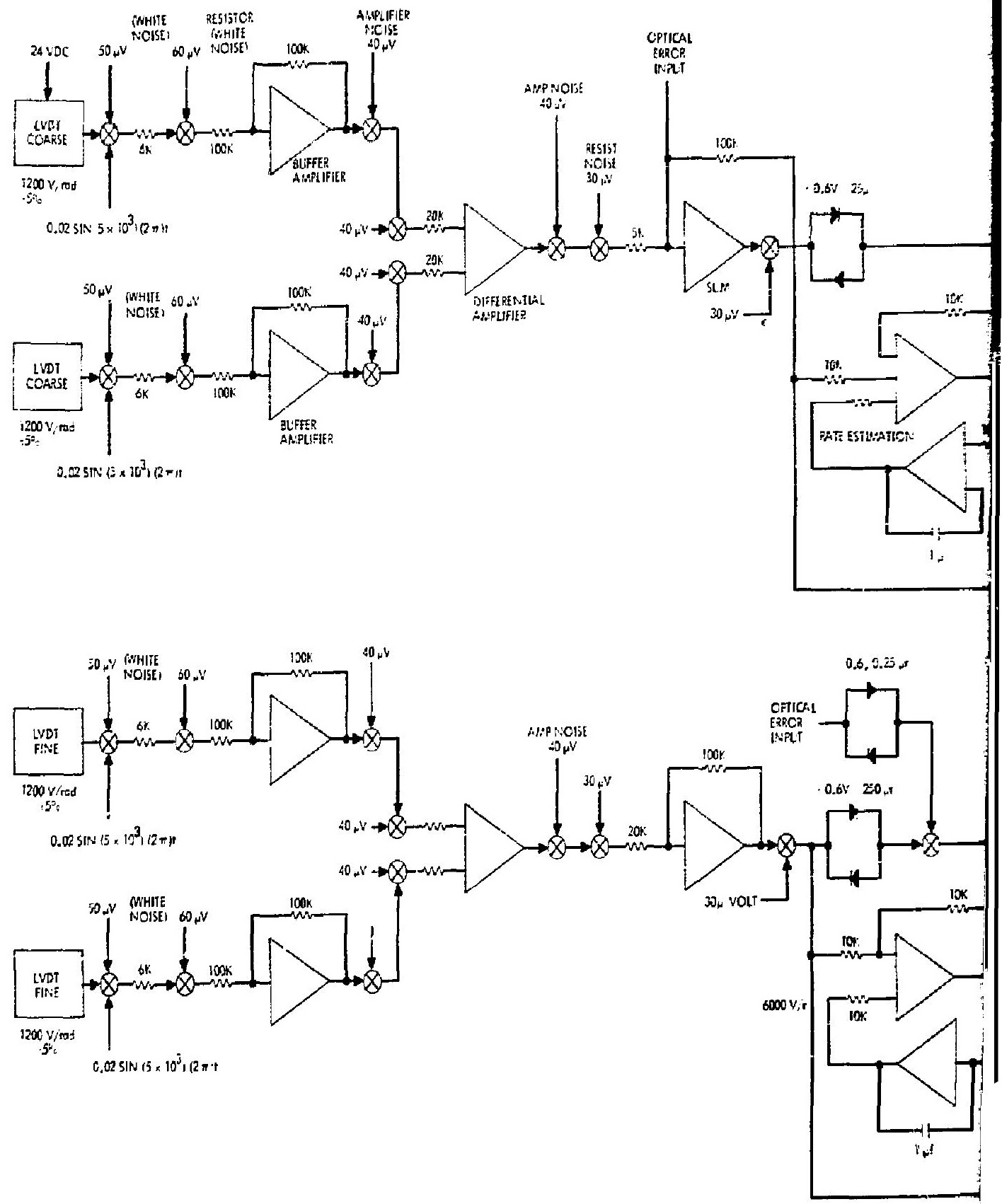


\section{SUMMARY OF CONCLUSIONS}

Various types of servo drive configurations have been evaluated. Since response time is not critical a spring ratio servo provides the required positioning resolution to $0.25 \mu \mathrm{rad}$. The LUDT selected provides position sensing to $10 \mu \mathrm{rad}$ which is within the coarse position resolution. By implementing simple control LRW the response approaches that of a step function command to the open loop spring inertia load. 


\section{REFERENCES}

1. Graham, D., D. T. MeRuser, Analysis on Nonlinear Control, J. Wiley and Sons, 1961, pp 375-418.

2. Pierre, D., Optimizalion Theory with Applications, J. Wiley and Sons, 1969, pp 501-512.

3. Higgins, R. A. Gorden, C. Hi., Control of Microinch Displacements. IEEE Trans, Ind. Elect. and Control Inst., Vol. IECI-20, No. 2, May 1973.

4. Miller, R. H., The Shaping Coil Displacement Transducer IEEE Conf. on Electromagnetic Components, 1971. 


\section{BIREFRINGENT SENSOR}

The bireiringent sensor described in this section is capable of sensing focus error, pointing error and wave front distortion. This sensor utilizes physical optic effects but must operate in the primary beam path. Therefore, it is proposid as a secondary sensor to be used as a totally independent measurement of alignment status. With this secondary sensor, the function of the primary sensors can be periodically checked or the alignment laser wavefront can be plotted as aid to beam diagnostics.

The in-focus criterion for this sensor occurs when the alignment beam is focused at the center of the surrogate target. When this condition is obtained, incoming and outgoing rays are coincident and parallel whether the incoming beam is collimated, divergent or convergent. See Figure 13-1.

This secondary or birefringent sensor is positioned in the beam and on the laser side of the final focusing lens. A polarization signature is imposed on the ingoing beam, a function of angle of passage. When the beam returns from the slirrogate target and focusing lens, a similar polarization signature is removed, also a function of angle passage. If the two angles of passage are exactly the same, the polarization signature is totally removed. A residual signature indicates a difference in augle between the ingoing and outgoing beams. This characte ri stic is illustrated diagramatically in Figure 13-2.

As illustrated by Figure 13-3, when positioned at the center of the focusing lens, this sensor ignoras focusing errors. In this position, it senses pointing errors only. At the outer edge of the focusing lens, it exhibits maximum sensitivity to focus error.

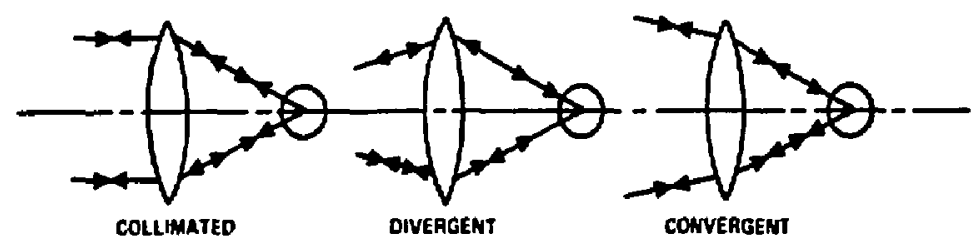

Figure 13-1. Parallel input-output rays focused at center of surrogate target 


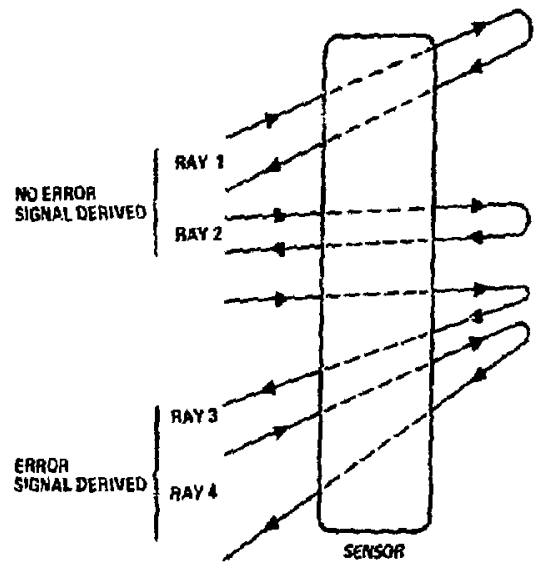

Figure 13-2. Sensor iunctional schematic

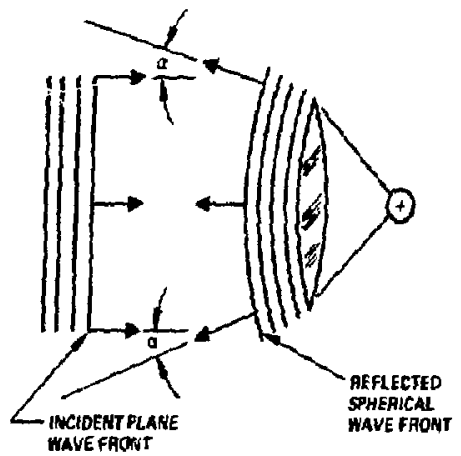

SENSOA WTH FDCUS EARDA

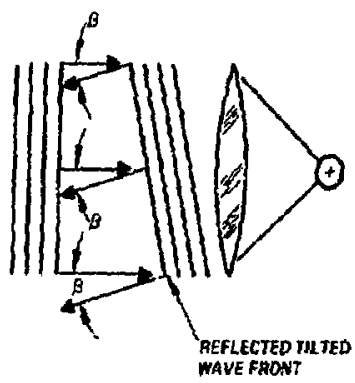

SENSOA WTH POWTHE ERAOA

Figure 13-3. Pointing and focus sensitivity as a function of sensor position

As illustrated in Figure 13-4, the sensor and its extrance /exit aperture are small compared to the final locusing lens. It is mounteo so that it can be traversed across the focus lens, and the traversing mechanism need not be precise. Precision ways are not required because the measurement made is the difference in angle hetween the ingoing and outgoing beams. 


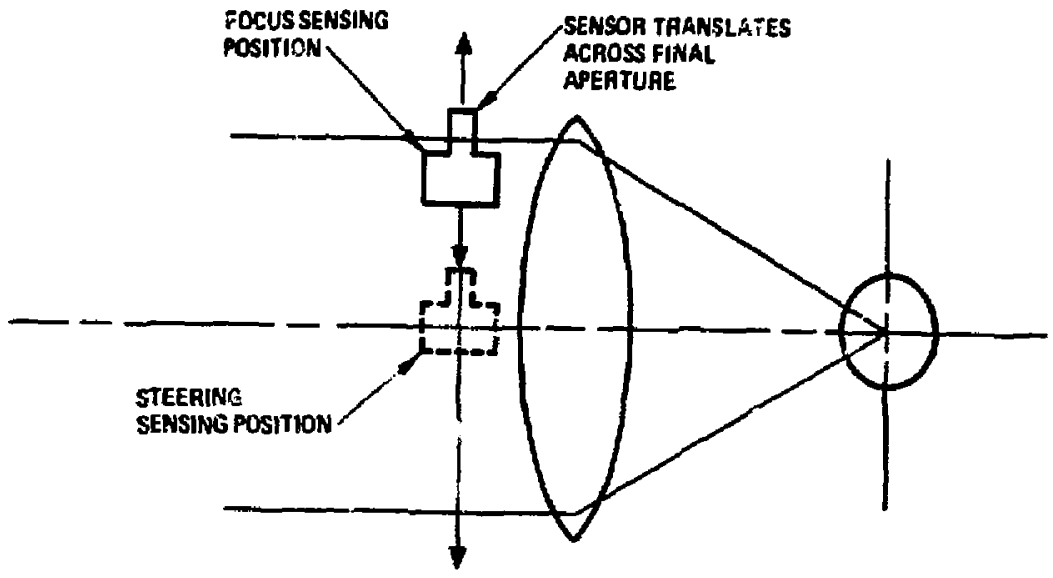

Figure 13-4. Strall sensor positioned in front of final aperture

The angle sensitivities required to sense a focus error of $\pm 7 \mu \mathrm{m}$ or a pointing error of $\pm 2 \mu \mathrm{m}$ are shown, in Figure $13-5$, to be $7 \mu \mathrm{rads}$ and $8 \mu$ rads respectively.

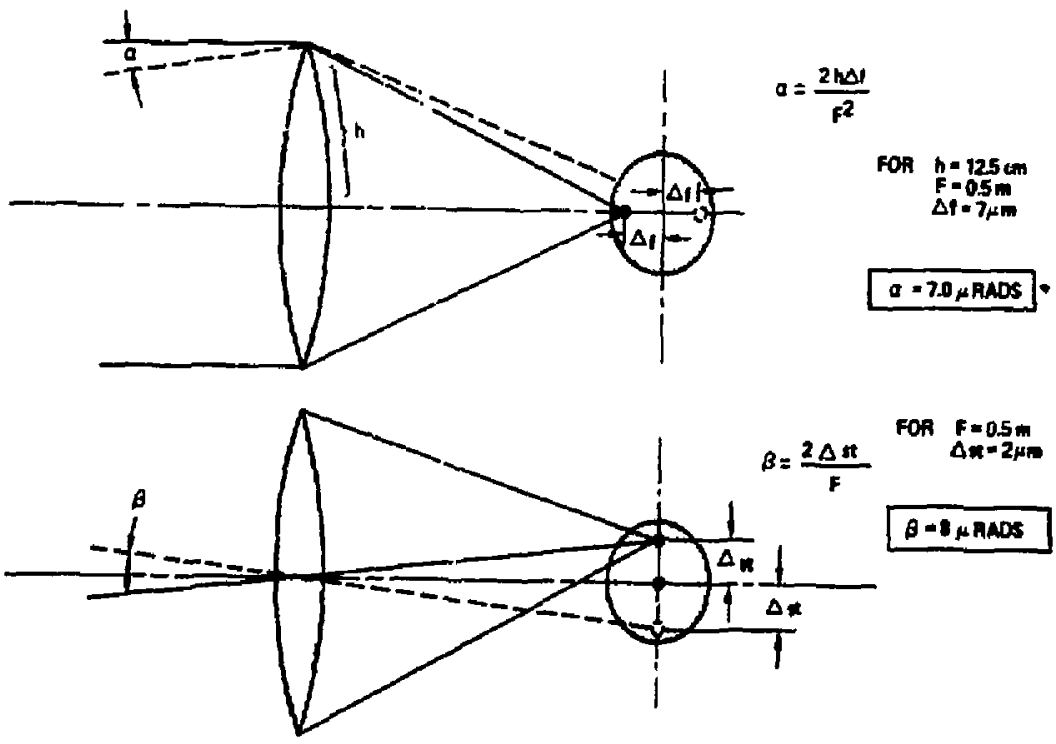

Figure 13-5. Return angle errors for surrogate target displacements 


\section{MEASURING ANGLE WITH BIREFRINGENT CRYSTALS}

Birefringent crystals such as quartz, calcite, and sapphire provide a basic tool for operating upon the polarization state of a light beam. The affect upon the polarization state by such crystals depends on:

1. Degree of birefringence (No-Ne)

2. Crystal thickness

3. Crysial orientation

4. Angle of incidence

With any given crystal, the first three parameters are fixed. The angle of incidence is the sole aeterminant of polariation state with passage through that crystal. As illustrated in Figure 13-6, a beam of polarized light, incident on such a crystal is divided into two components which are polarized orthogonal to one another. During paseage through the crystal, one component travels at a greater velocity than the other and causes an ever increasing relative phase difference betveen the two components. Upon emergence irom

PASSAGE THAOUGH A BIREFRINGENT CAYSTAL CHANGES THE POLARIZATION STATE OR RELATIYE RETARDAHICE. THIS CHANGE IS A FUNCTION OF

- Caystal biREFringence ( $\left.N_{0} \cdot N_{e}\right)$

- caYstal thICKNESS (t)

- caYstal oqientation

- ANGLE OF INCIDENCE (i)

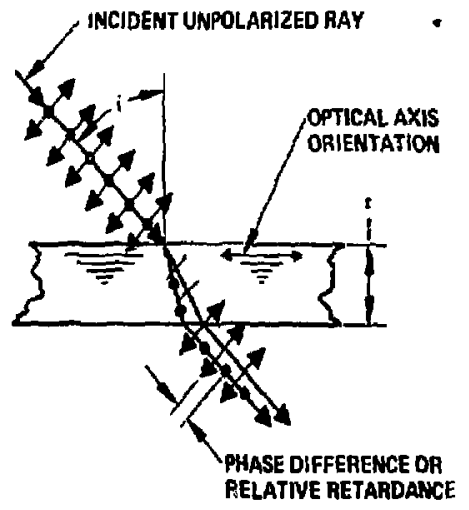

Figure 13-6. Angle measurement by polarization change 
the crystal, these out-of-phese components recombine to fo:m some form of eliptically polarized light.

As illustrated in Figure 13-7, the ray velocity surface permits visualization of light propagation velocities in all directions of a given crystal.

Light energy following any ray $O P$ consists of two wave trains: one vibrates in the principal section (plane of figure) and contains extraordinary waves. The other is perpendicular to the principal section and contains ordinary waves.

In three dimensions, the velocity of the ordinary wave moving along all the rays is represented by a sphere. The extraordinary ray velocities are represented by an ellipsoid of revolution.

The retardation characteristic as a function of angle of passage of any given birefringent element car be directly viewed by placing the element between crossed polarizers. The observed line patterns represent families of passage angles that provide equal retardation. They have beeni variously called isogyros, isopleths, interference fringes, and isochromatic patterns when viewed in monochromatic light,
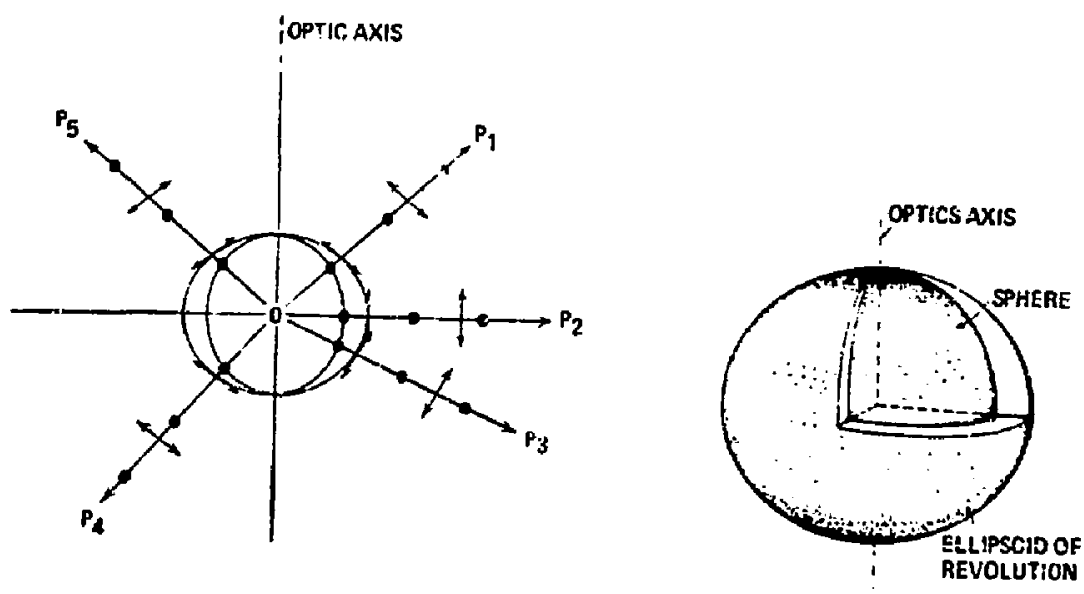

PAIMTIPAL SECTION OF NEGMTIVE RAY.VELOCITY SURF ACE

UNIAXIAL NSGATIVE RAY.VELCCITY

SURFACE SECTIONED TO SHOW SPHERE WITHIN AN ELLIPSOID OF REVDLUTION.

Figure 13-7: The ray-velocity surface 
Figure 13-8 shows the pattern oi a birefringent plate between crossed polarizers with the optical axis parallel to its principa! faces. A beam passing through at normal incidence is represented at the center of the pattern. An incident angle $\theta$ is represented by a circle of sone radius $r$ about the center. Such a simple retarder would encode the angle of incidence onto the polarization of a passing beam but in an ambiguous manner. Angles of incident as measured frols the elovation and azimuth planes cannot be differentiated.

\section{SAVART PLATE}

The isochromatic pattern that will provide sensitivity about only one axis consists of a series of parallel and straight lines. Such a pittern is closely approximated by the Savart plate, which is actually an assembiage of two birefringent plates as shown in Figure 13-9.

It is formed by cutting two plane parallel plates of uniaxiai crystal of equal thickness in such a way that the normal of these plates makes the same angle with the optical axis of the crystai. This angie is, most often, approximately 45 degrees. The two plates are then mounted to one another after one is turned 90 degrees witi respect to the other.

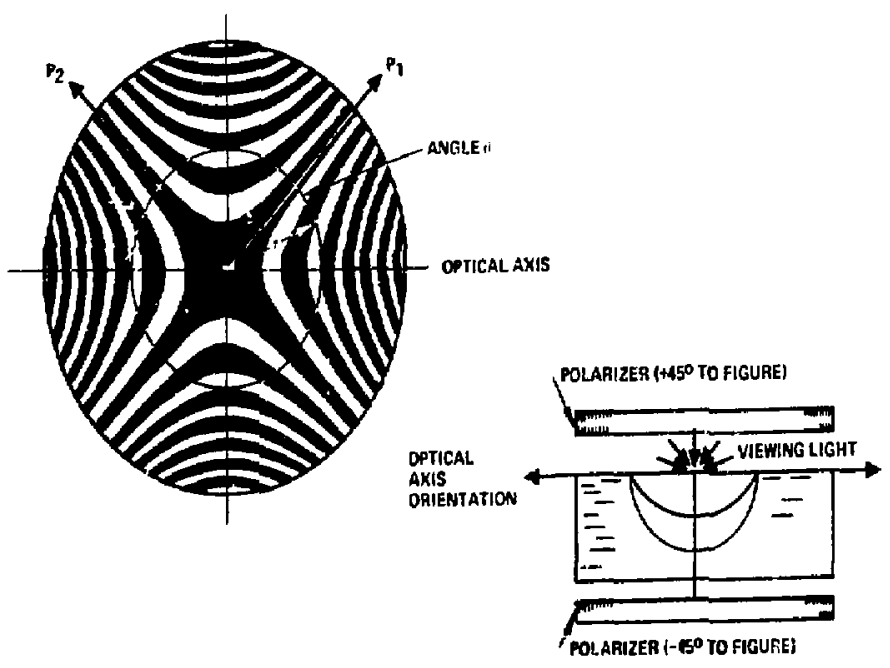

Figure 13-8. Isch romatic pattern seen through crossed polarizers 


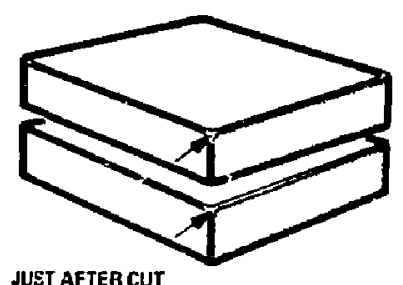

SAVART PLATE COASTRUCTION
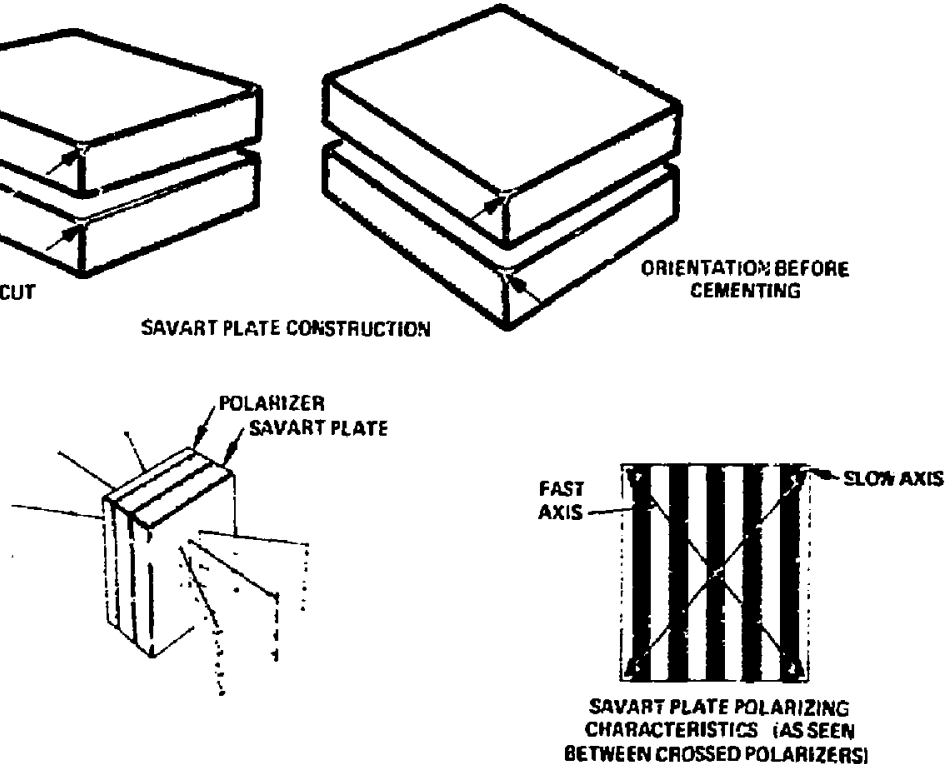

Figure 13-9. Savart plate construction and characteristics

Notice that as a beam of light is tilted about the sensitive, or vertical, axis the relative retardation changes. However, as the beam is tilted about the insensitive, or horizontal axis, no change in relative retardation occurs. Thus, a Savart plate encodes the angle of incidence only about a single axis onto the polarization of the beam. It is unambiguous if the FOV is restricted to the region of a single fringe. The fringe spacing is controlled by the thickness of the plate.

In the figure, the polarizer is vertical and the fast and slow axes of the Savart plate are ti 45 degrees to the vertical. The emerging polarization is unchanged for normal incidence, so the central fringe is linearly polarized in the vertical direction. At the other angles of incidence, the polarization assumes various forms of elliptical polarization. 
A iirst order approximation of the angrilar distance between minima, or the angular spacing which causes a relative retardation of one wave for a Savart plate of thickness $t$, is equal to $\mathrm{N} / \mathrm{g}$ where $\mathrm{g}$ is:

$$
\mathrm{g}=t \frac{\mathrm{No}^{2}-\mathrm{Ne}^{2}}{\mathrm{No}^{2}+N \mathrm{Ne}^{2}} \sqrt{2}
$$

In Figure 13-10 the relative retardation imposed by $20 \mathrm{~mm}$ thick Savart Plates of various materials is plotted as a function of angle of incidence expressed in $\mu$ radians. Signal strength expressed as a factor of the total power passing through the sensor is also shown as a function of incident angle. It can be seen from this figure that a $20 \mathrm{~mm}$ thick sapphire plate sill provide a maximan signal change when a beam is at an angle of incidence of $800 \mu$ rads.

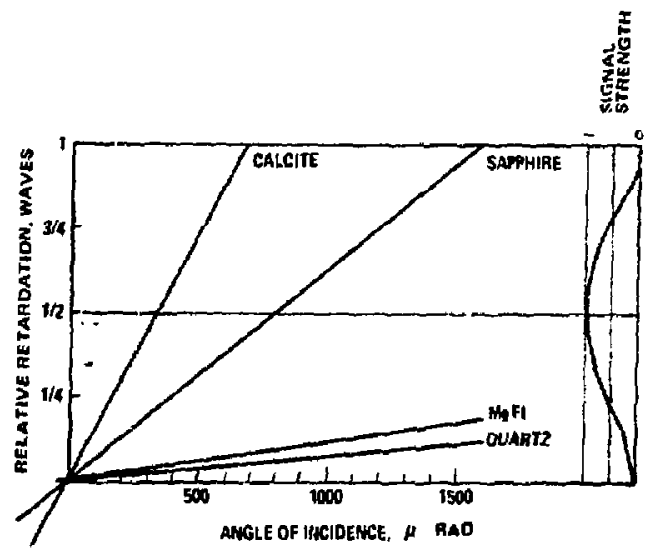

\section{Figure 13-10. Relative retardation and signal strength as a function of angle of incidence $120 \mathrm{~mm}$ thick Savart plates)}


Table 13-1 tabulates the degree of birefringence, cost and potential sources for the most prominent candidate Savart Plate materials. From the information in this table and the preceding paragraph, sapphire appears to be the material of choice.

\section{PRINCIPLE OF OPERATION}

With reference to the optical schematic of Figure 13-11, the radiant energy from the alignment laser is first converted to circularly polarized energy with the linea" polarizer and the quarter wave plate located at the first entrance aperture of the sensor. Converting to circular polarization provides three separate functional advantages:

1. The Savart Plate, and hence the sensor, need not be precisely aligned about an axis paraliel to the optical axis of the laser (or the laser's propogati, in direction).

2. It provides a convenient polarization reference state. This will be discussed in later paragraphs.

3. It biases the signal $\mathrm{vs}$ angie change curve $1 / 4$ wave and in so doing places the null or zero error point where the signal change $v s$ angle change is a maximum. Set Figure 13-10, signal strength as a furction of angle of incidence.

Table 13-1. Potential Savart Plate material summary

\begin{tabular}{|c|c|c|c|c|c|}
\hline MATERIAL & $\begin{array}{c}A T=1 \\
N_{0}\end{array}$ & $\begin{array}{c}1.06 \mu \mathrm{MH} \\
\mathrm{Nk}\end{array}$ & git & SCOST. SIZE & SOURCE \\
\hline SAPPHIAE & 1.7555 & 1.8355 & 0.063 & $\begin{array}{l}\text { S413 } \\
\text { 3N. DIA }\end{array}$ & MEI LER \\
\hline CALCITE & 1.6428 & 1.4798 & 0.147 & $\begin{array}{l}\$ 1300 \\
21 \mathrm{~N} .50\end{array}$ & $\begin{array}{l}\text { CAYSTAL } \\
\text { OPTICS }\end{array}$ \\
\hline OUARTZ & 1.5328 & 1.5415 & 0.0079 & $\begin{array}{l}5400 \\
1.5 \mathrm{in} . \mathrm{so}\end{array}$ & $\begin{array}{l}\text { CAYSTAL } \\
\text { OPTICS }\end{array}$ \\
\hline$m_{2} F_{2}$ & 1.3760 & 1.3077 & 0.0119 & $\begin{array}{l}500 \\
3.5 \mathrm{M} \text {. DIA }\end{array}$ & HARSHAW \\
\hline
\end{tabular}




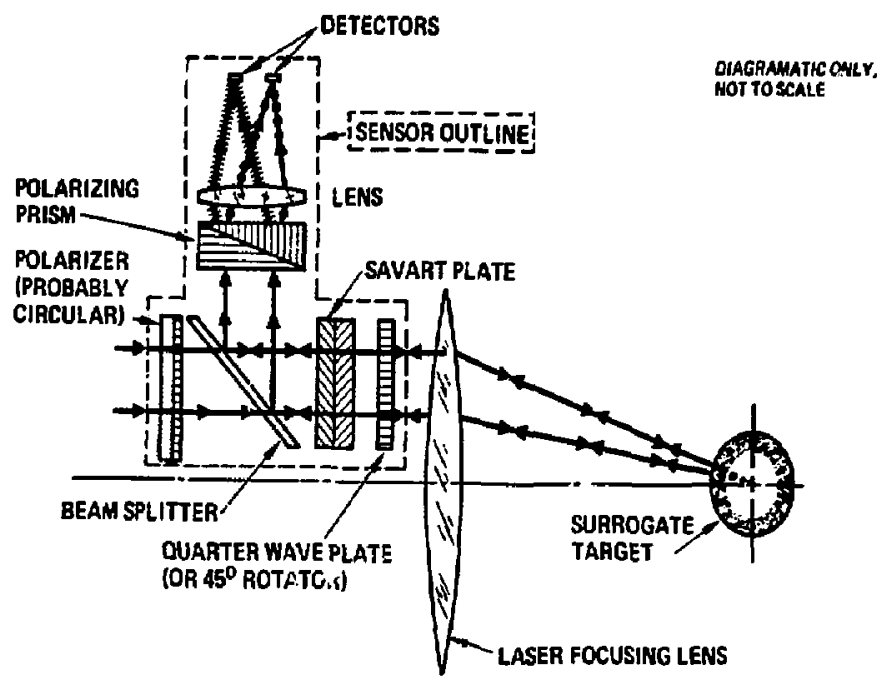

Figure 13-11. Sensor cptical schematic

The circularly polarized energy then passes through a beam splitter and then through the Savart Plate with an angle of incidence which is approximately zero. The circularly polarized light is divided, by the Savart Plate, into two equal and in-phase orthogonal components. For the purpose of this discussion we will assume the se linearly polarized components to vibrate in planes which are at $45^{\circ}$ to the plane of the Figure 13-11. Since the energy was not perfectly perpendicular to the Savart Plate, one of the two cotnponents is retarded with respect to the other during passage through the Plate as described in the paragrapi above. This relative retardation is the polarization signature imposed on the emerging beam.

The quarter wave plate is next encountered. It's action is to lafter two transversals -- ingoing and outgoing) rotate each of the two orthogonal components by $90^{\circ}$. When this is done, the component which was retarded the greatest amount upon the ingoing traversal is in a position to travel the fastest during the outgoing traversal.

Now the relative retardance or polarization signature imposed by the first traversal will be cancelled by the second traversal when, and only when, 
their angles of incidence are identical. As discussed in the opening remarks of this section, this will only occur when the alignment laser energy is focused at the center of the sphere.

When the relative retardance imposed by the two traversals exactly cancel, the original polarization tate is achieved, a fact which $c$ an be made easily discernable.

A lack of parallelism between the ingoing and outgoing beams causes a residual relative retardance which results in some form of elliptically polarized light. This too, can be rendered easily discernable.

\section{ERROR DETECTION AND ANALYSIS OF POLARIZATION STATE}

In the following paragraphs the proposed method for detecting the residual retardance which represents the lack of parallelism between an ingoing and outgoing beam is described. In this discription recovrse is made to a visualization tool known as the Poincare sphere. The Poincare sphere, conceived by Henri Poincare about 1892, provides an exceedingly, convenient way of representing polarized light and predicting the effect of any giver retarder or combinations of retarders and at any orientation. For these reasons as well as its slightly caballistic nature, the Poincare sphere will be described first.

\section{Poincare Sphere}

Poincare's sphere is a mapping method: Each point an the sphere represents a different polarization form. The mapping may be carried out with the aid of a three-dimensional model, a two dimensional projection, trigonometry, or analytic geometry. The method is applicable only if the incident beam is completely polarized which is the case in the sensor under discussion bere.

With Reference to Figure 13-12 each point on the sphere represents a different polarization form. The upper and lower poles represent left and right hand circularly polarized light, respectively. Points on the equator indicate linear polarization and other points indicate elliptical polarization with the direction of their major axes determined by the longitude of the 


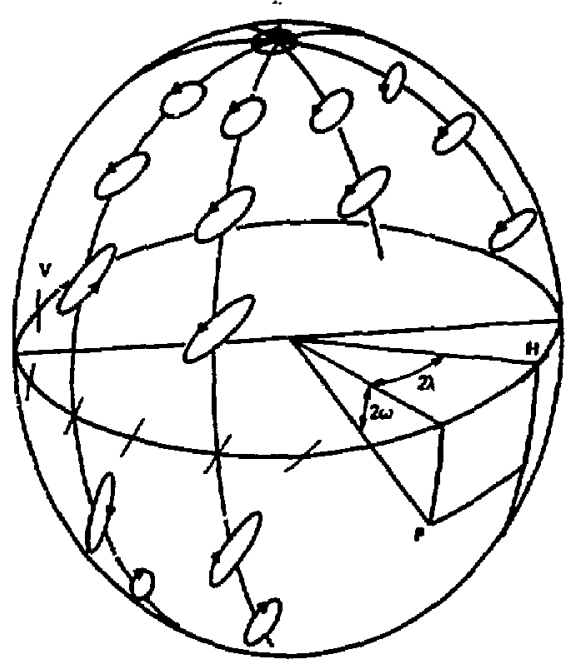

Figure 13-12. The Poincare sphere, showing the general significance of the different areas and the specification of the general point $P$ in terms of the angle $2 \lambda$, meas ured clockwise from $H$, and $2 \omega$, measured downward from the equator.

points. An arbitrarily chosen point on the equator designates horizontal polarization. Orthogonal polarization directions are diametrically opposed. Usually the radius of the sphere is assumed to be unity: sometimes it is regarder as proportional to the intensity of the beam.

A general point $P$ on the surface of the (unit-radius) Poincare sphere is specified in terms of the longitude $(2 \lambda)$ and the latitude $(2 \omega)$, where: -180 degrees $\leq 2 \lambda \leq+180$ degrees and -90 degrees $\leq 2 \omega \leq+90$ degrees. The longitude is positive when measured clockwise from point $H$; the latitude is positive when measured downward from the equator, that is, toward the pole representing righthand circularly polarized light.

The general point $P$ represents a completely polarized beam whose ellipse has azimuth $\lambda$, ellipticity $\tan |\omega|$ and is either lefthanded or righthanded according to whether $P$ lies in the upper or lower hemisphere. In 
summary, $P$ represents an elliptically pclarized beam whose sectional pattern is described thus:

$$
\begin{aligned}
a & =\lambda \\
b / a & =\tan |w|
\end{aligned}
$$

where $b$ and $a$ are the major and minor axes of the elliptically polarized light.

To determine the effect of inserting a retarder into a polarized beam one merely constructs an arc or circle on the sphere perhaps with a draftsman's compass. The center of the arc is chosen to be the azimuth orientation of the retarder's fast axis. The beginning of the arc is selected as that point which represents the polarization state of the incident beam. The arc length depends upon the retardance of the retarder, a quarter wave retarder requiring a $90^{\circ}$ arc length. Finally, the end of the arc represents the new polarization state of the emerging beam.

As an illustration of the method, consider a beam of light that is linearly polarized at an azimuth of $45^{\circ}$, as indicated by point $P$ in Figure 13-13 and suppose that the bear. encounters a linear retarder whose retardance is one quarter wave and whose fast axis is at $22.5^{\circ}$ as indicated by $R$. Then the pertinent rotation is indicated by the righthanded, arc that starts at $P$ and is generated by a $90^{\circ}$ rotation about the radius vector through $R$. The end point $P$ is the answer: It indicates the polarization form of the emerging beam. Clearly the beam is left elliptically polarized ibecause $P$ lies in the upper hemisphere); the major axis is at $22.5^{\circ}$ (because $P$ lies on the same meridian as $R$, namely the meridian for which $2 \lambda=45^{\circ}$ ); the ellipticity is $\tan 22.5^{\circ}=$ 0.4142 (because $2 \omega=45^{\circ}$, and hence $|\omega|=22.5^{\circ}$ ).

If the linearly polarized incident beam were to have a vibration direction that differs by $45^{\circ}$ from the $90^{\circ}$ retarder's fast axis, the end point of the arc would necessarily be at a pole; hence the use of $90^{\circ}$ retarders in converting linearly polarized light to circularly polarized light. 


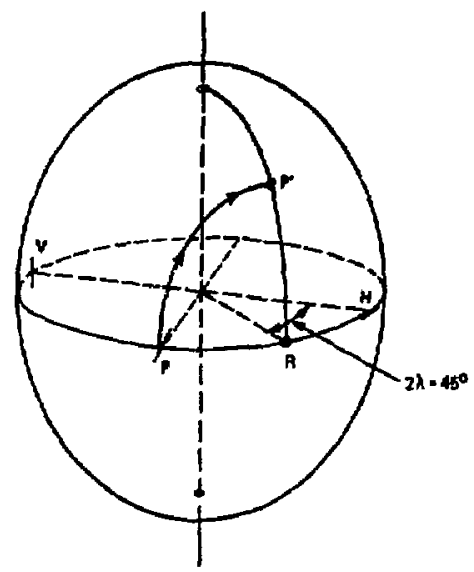

Figure 13-13. Use of the Poincare sphere in predicting the effect of a $90^{\circ}$ linear retarder $\mathrm{R}$ (with tast axis at $\left.22.5^{\circ}\right)$ on a linearly polarized beam $\mathrm{P}$ (with vibration direction at $45^{\circ}$ )

\section{Poincare Analysis of Birefringent Senaor}

Figure 13-14 presents two views of the Poincare sphere with polarization states, crystal orientation and arcs which represent a typical ray as it passes through the sensor.

The incident alignment laser energy is first vertically polarized by $P$. It then passes ihrough the first quarter-wave plate (N/4), and is circularly polarized as indicated by arc (1). It then passes through the Savart Plate, SP, at some angle other than normal and undergoes a relative retardance of $\delta$. (2) illustrates the arc which creates the resultant polarization state: elliptical with major axis horizontal.

The second quarter-wave plate $(N / 4)_{2}$ is next encountered. The energy is linearly polarized as indicated by arc (3) and oriented at an angle from vertical equal to $\left(45^{\circ}+S / 2\right)$.

After reflection from the surrogate target, the illustrative beam panses throigh the second quarter-wave plate $(\lambda / 4)_{2}$ for the second time and is 

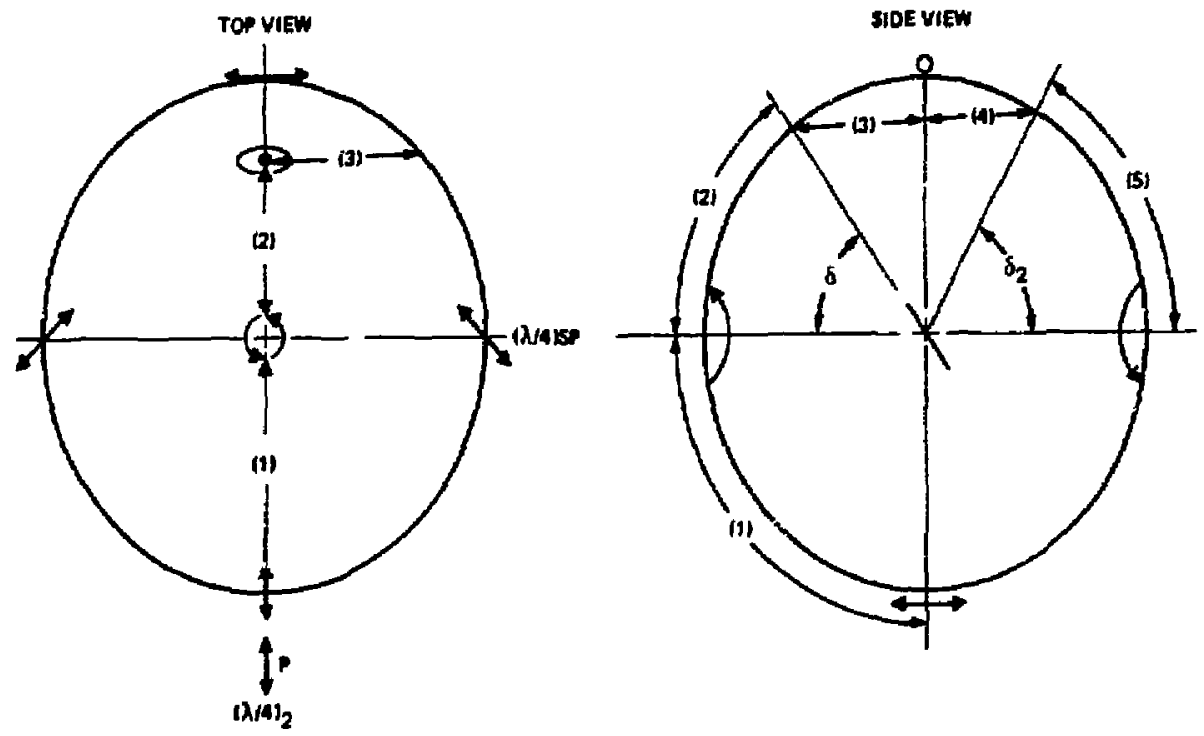

Figure 13-14. Poincare sphere analysis of birefringent sensor

converted to elliptical similar to its's earlier elliptical state but with a "handerness" opposite to before. Arc (4) illustrates this conversion.

The second passage through the Savart Plate next occurs and in this example we assume perfect parallelism and hence perfect cancellation. $\delta$. is equal to $\delta_{2}$. Arc (5) shows that circular polarization is again obtained although the handedness is opposite to the original state.

After reflection from the beamsplitter the beam passes through the polarizing prism, which resolves the polarized energy into two orthogonal polarization states, vertical and horizontal. Two detectors are used to sense the intensity ratio between the two beams. A balance (as illustrated) indicates focus or parallelism between the ingoing and outgoing beams. 


\section{OPTICAL ANALYSIS FOR SAVART PLATE SENSOR}

The Savart plate sen:0i unit derives an error signal when any beam traversing it from left to right returns at any angle other than parallel to its original direction. The concept is shown schematically in Figure 13-15. For the autoreflection condition shown for the perfect case (Case 1. Figure 13-15), the error is zero. For cases with misalignment. ( $\triangle \mathrm{Y} \neq 0)$, or focusing error, $(\Delta \mathrm{Z} \neq 0)$, the sensor unit detecto these errors by sensing the elevation and azimuth components of $\Delta \alpha$. These components, $\Delta \alpha_{E I}$ and $\Delta \alpha_{A Z}$, are defined in Figure 13-16.

As shall be shown later (see Figures 13-18 and 13-19), there is a definite correlation between these angular components, $\left(\Delta \alpha_{A Z}, \Delta \alpha_{E L}\right)$, and the alignment errors $(\Delta Y, \Delta Z)$. And this correlation makes it possible to devise a procedure for reducing these errors independently. Furthermore, this procedure will be valid even for tine case with aberrated input wavefrort.

1. meafect case

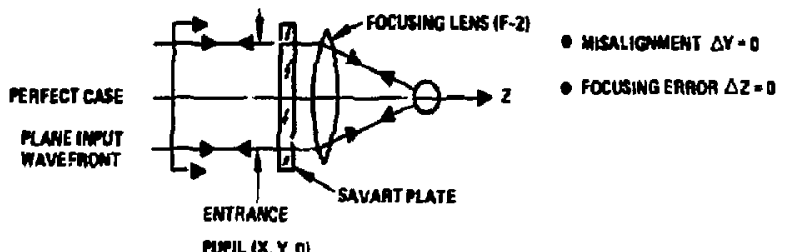

- Misalioment ar.o

2. CATE WITH $\Delta Y+0, \Delta Z+0$

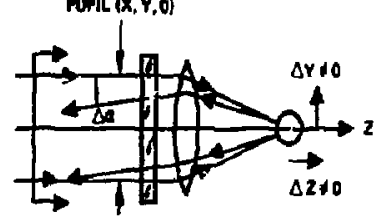

$\Delta \mathbf{\Delta}+0$ AlankATEDINYUT

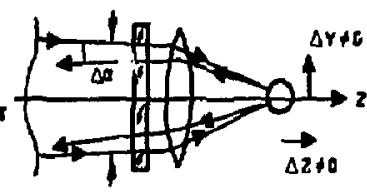
SAYART PLATE SELOR UWT Derects Sa elevation $\Delta{ }^{\circ}$ aznuvith

Figure 13-15. Savart plate sensor unit for detecting misalignment and focusing errors 


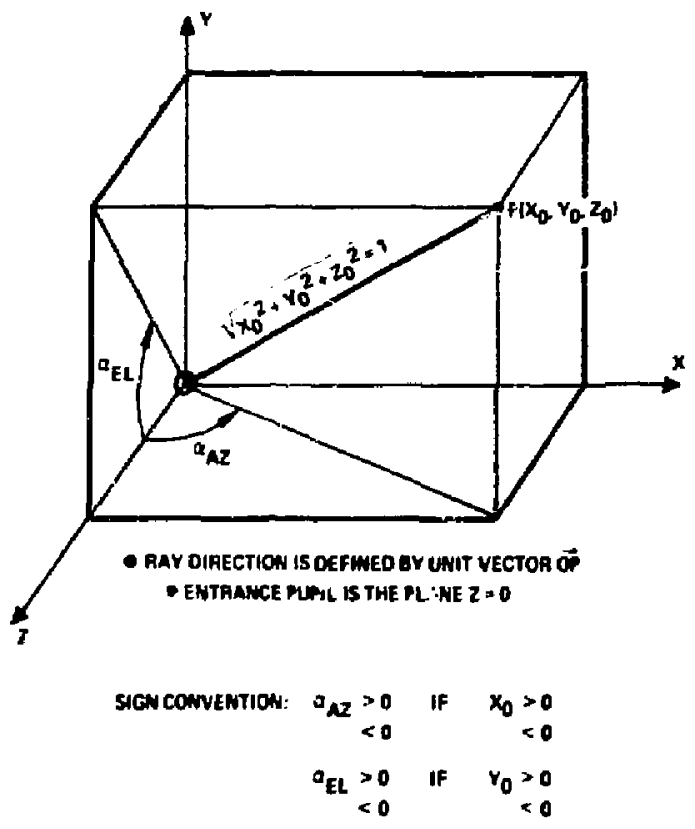

$$
\begin{aligned}
& \Delta a_{A Z}=1 a_{\text {AZ }} l_{\text {AETUAN }} \cdot a_{\text {AZ }} \text { MUNOT } \\
& \left.\Delta a_{E L}=\left.1 a_{E L}\right|_{R E T U M N}-\mid a_{E L}\right\}_{\text {IWUUT }}
\end{aligned}
$$

Figure 13-16. Definitions of $\Delta_{E L}$ and $\Delta_{A Z}$

The input wavefront aberration is simulated by making use of the aberration function, $W(r, \theta)$, defined in Figure 13-17. This function is expressed in polar coordinates so that the computed $\Delta a^{\prime} s$ can be plotted as a function of $r$ for range of $\theta$ values.

For the case with perfect input wavefront, Figure 13-18 shows the correlation between the $\Delta \alpha^{\prime} s$ and misalignments, $(\Delta Y, \Delta Z)$. The graphs on the left show that $\Delta \alpha_{E L}$ and $\Delta \alpha_{A Z}$ curves oscillate about the line $\Delta \alpha=0$ vinen both $\Delta \mathrm{Y}$ and $\Delta \mathrm{Z}$ are zero. This oscillation is due to the residual spherical aberration of the focusing lens. The graphs in the middle show that, in the presence of a focusing error $(\Delta Z=-7 \mu \mathrm{m})$, but no misalignment, $\Delta Y$, the curves fan out as $x$ increases. Note that, $\left[{ }^{\Delta \alpha} \alpha_{A Z}\right]_{r=0}=\left[\Delta \alpha_{E L}\right]_{r=0}=0$. 


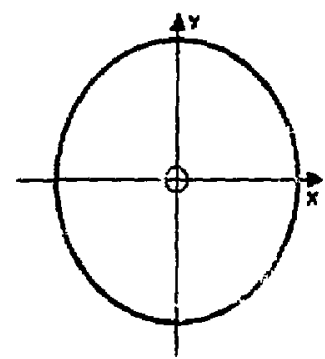

EHTRANCE PUNIL (K, YI

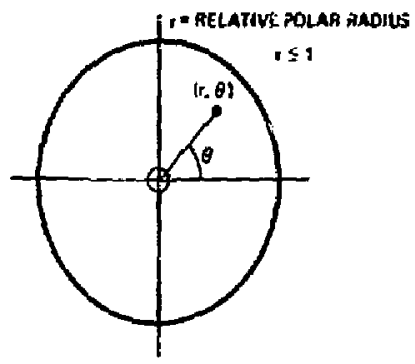

ENTRAKCE MUIL $(1,0)$

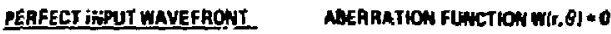

AOERRATED IMUUT HAVEFROHT $\left.\quad W(r . \theta)=w_{22} t^{2} \tan ^{2} \theta-\sin ^{2} \theta\right)+w_{31} r^{3} \sin \theta+w_{40} r^{4}$

$W_{22}=$ ASTIGMATISH $(14 \lambda)^{*}$

$v_{31}=\cos 4(1,4)$

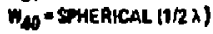

"Half of the peak to peak value

Figure 13-17. Input wavefronts for Savart plate study

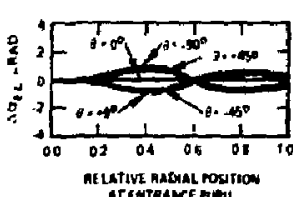

ar ENTRAMCE nient

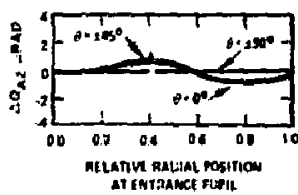
at fHT RALCE Fül! $i_{i=0}=0$

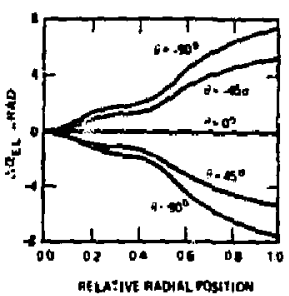

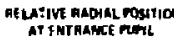

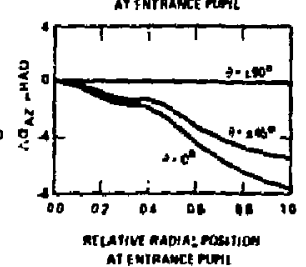

$x_{i=0}^{x \rightarrow \infty} \times$

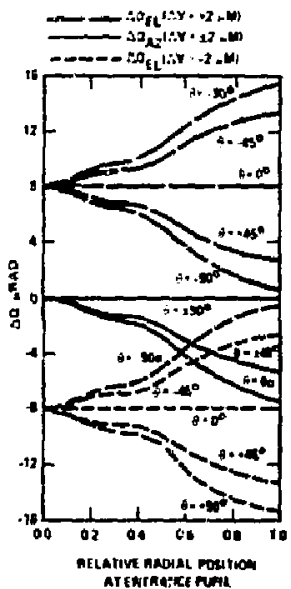
$\sin =12=4$

Figure 13-18, $\Delta \alpha$ as a function of relative radial position at entrance pupil perfect input wavefront

The grephs on the right show that in the presence of both a focusing error and misalignment, $(\Delta \mathrm{Y}= \pm 2 \mu \mathrm{m})$, the curres not only fan out but $\left[\Delta_{E}{ }_{E}^{-}\right]_{r=0} \neq 0_{1}$ even though $\left[\Delta \alpha_{A Z}\right]_{r=0}=0$. (If misalignment is in the $X$-direction, the reverse will be true.) 
Therefore, by reducing $\Delta \alpha^{\prime} s$ at or near $r=0$, misalignment can be mimized. On the other hand, by reducing the differences of $\Delta a^{\prime} s$ for $0 \leq r \leq r_{\text {max }}$ locusing errors can be eliminated.

With the aberrated input wavefront present $t_{t}$ the $\Delta$ a curves take the shapes as shown in Figure 13-19. $\mathrm{T}^{\prime}$ : general characteristics of the se curves are very similar to those with perfect input wavefront. Thus the same procedure of minimizing $\Delta Y$ and $\Delta Z$ applies. Indeed, by comparing the graphs on the left between Figure 13-18 and Figure 13-19, the absolute values of residual $\Delta \alpha^{\prime} s$ are almost identical.

\section{BIREFRINGENT SENSOR DESIGN}

Figure 13-20 illustrates a preliminary mechanical layout of the birefringent sensor. The sensor can be indexed to any point along a diameter of the focus lens aperture via the Acme screw and stabilizer rod.

Anyone of two hifserent thickness Savart Plates can be indexed into position thus providing two different sensitivities

The $90^{\circ}$ rotation driver permits angles to be measured in azimuth or elevation.
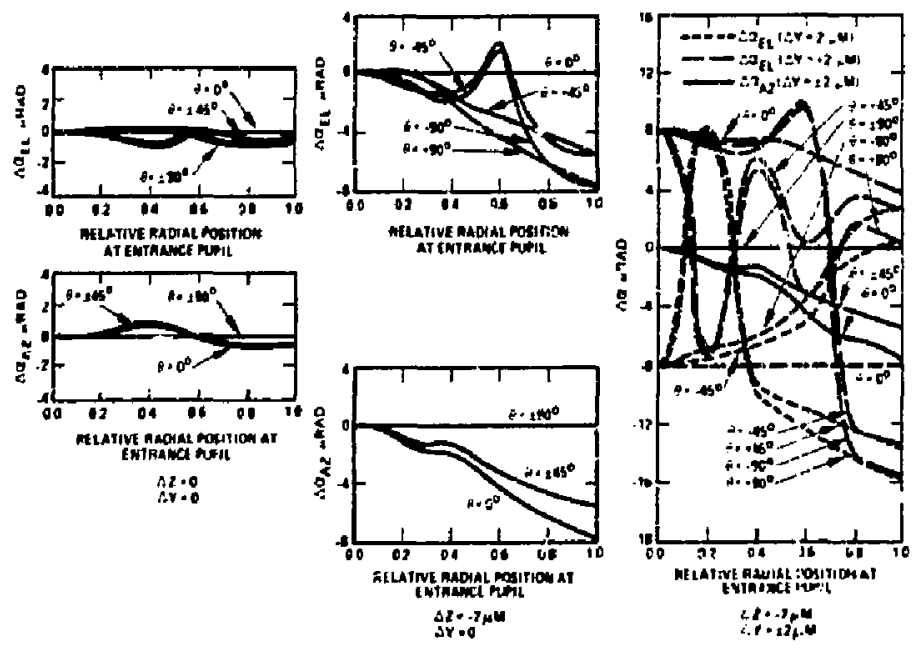

Figure 13-19. $\Delta \alpha$ as a function of relative radial position at entrance pupil. Aberrated inpuwavefront (1/2 $\lambda$ coma, $1 / 2 \lambda$ astigmatism, $1 / 2 \lambda$ spherical) 


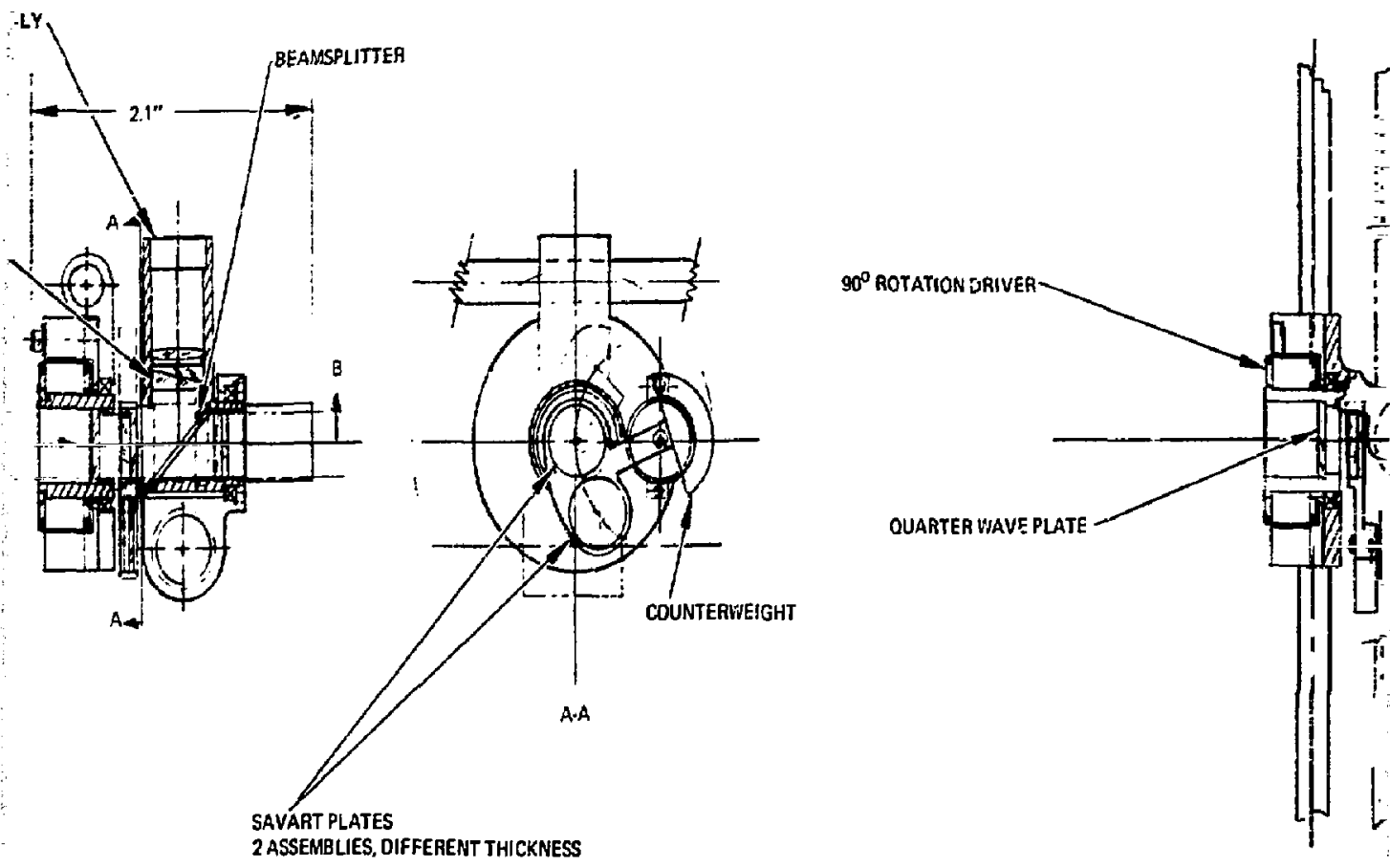


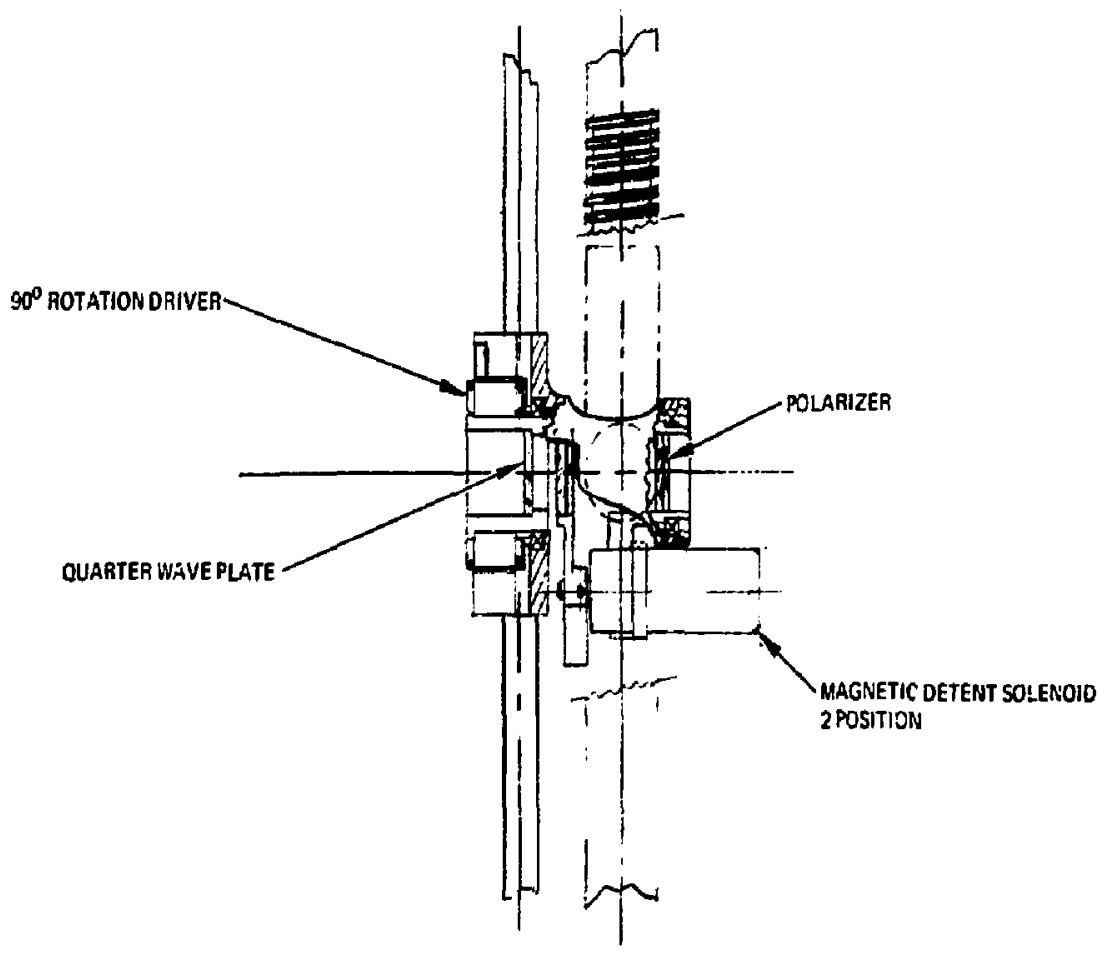

Q-B

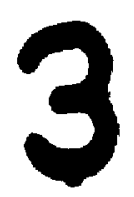

Figure 13-20. Proliminary Mechanical layout of the birof ringent sensor 